\title{
Developing and investigating validity of a knowledge management game simulation model
}




\section{Doctoral committee}

Chair:

Prof. dr. H.W.A.M. Coonen

Promotor:

Prof. dr. R. de Hoog

Members:

Prof. dr. A.J.M. de Jong

Prof. dr. W.R. van Joolingen

Prof. dr. R.J. Tissen

Prof. dr. J. van Dijk

Dr. A.B.M. Wijnhoven

Tsjernikova, Irina I.

Developing and investigating validity of a knowledge management game

simulation model

Ph.D Thesis, University of Twente

Print: Ipskamp Drukkers B.V., Enschede, The Netherlands

Cover design: Luuk Vosslamber

ISBN: 978-90-365-2915-0

(C) 2009, Irina I. Tsjernikova, Enschede, The Netherlands 


\title{
DEVELOPING AND INVESTIGATING VALIDITY OF A KNOWLEDGE MANAGEMENT GAME SIMULATION MODEL
}

\author{
PROEFSCHRIFT
}

ter verkrijging van

\begin{abstract}
de graad van doctor aan de Universiteit Twente, op gezag van de rector magnificus, prof. dr. H. Brinksma, volgens besluit van het College voor Promoties

in het openbaar te verdedigen

op donderdag 29 oktober 2009 om 16.45 uur
\end{abstract}

door

Irina Ivanovna Tsjernikova

geboren op 31 oktober 1969

te Moskou 
Dit proefschrift is goedgekeurd door de promotor:

Prof. dr. R. de Hoog 


\section{Acknowledgements}

Writing this small, but important and pleasant part of this book means that one of the goals in my life is reached. Achieving this goal without contribution and support of many people was not possible. I would like to express my deep gratitude to all people who helped me to accomplish this goal.

First of all I would like to thank Ton and Robert for encouraging me to finalize this thesis. Robert, I am completely aware that being my supervisor took a lot of energy from you. I am very grateful for your efforts and patience. Discussions with you, your critical questions and intellectual stimulation allowed me to find a way to combine different science fields together and complete this research project. Thank you for helping me to become a better researcher.

Work on this research started many years ago within the KITS project. It was very exciting and encouraging to work with people from different countries and settings. I would like to thank all members of the KITS team for this experience. Robert de Hoog, Ton de Jong, Henny Leemkuil, Noor Christoph, Rijanto Purbojo, Susanne Ootes, Anjo Anjewierden, and Jakob Sikken from the KITS team contributed to the work on the simulation model. I owe special appreciation to Anjo and Jakob. Anjo, without your contribution to my thinking and your KMsim Tool the model would never be implemented. Jakob, thank you for your assistance in implementing the model and helping me with the environment during the experiments.

Further, my sincere gratitude goes to the group of experts participated in this research project. Your contribution to this work was very valuable and I appreciate your expertise very much.

I am very thankful to Paul Hendriks from the Radboud University in Nijmegen, who was a pioneer in using the KM Quest game in teaching and who provided me with the opportunity to conduct experimental studies in Nijmegen. Paul, it was a pleasure to work with you.

My fellow-colleagues in the IST department thank you for fruitful discussions during the ProIST meetings and for being a good company through these years. Susanne, Rijanto, and Sylvia, you were the 'lucky' victims of my sudden ideas and questions. Thank you for being the perfect roommates. Larisa and Daphne, thank you for explaining me some features of Word.

Coming to live abroad means meeting new people and making new friends. Adriana \& Andrei, Dragana, Kira, Alona and Lena, Oksana \& Sasha, Nelly, Zamira, Tanya, Lilit $\&$ George, Lilia thank all of you for making my life here colorful. I appreciate endlessly your help, support and readiness to be with me in bad and good moments.

Finally, I would like to thank my family. My dear parents, Ivan and Valentina, my sister Katja, Olga and Wim thank you for your love, help, understanding and confidence in me. Olga, sometimes you behave like a mother to me. Thank you for your care. Wim, your love, care and support made it possible to bring this thesis to an end.

Irina Tsjernikova,

September 2009 , Enschede 



\section{Table of contents}

\section{Chapter 1}

What is this book about? ..............................................................................................1

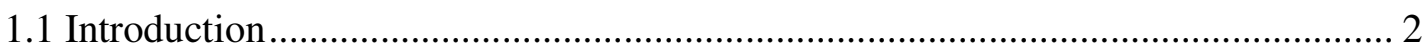

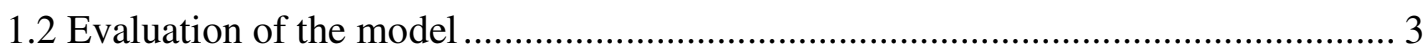

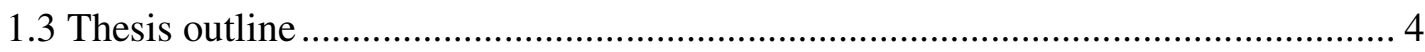

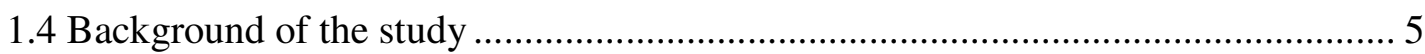

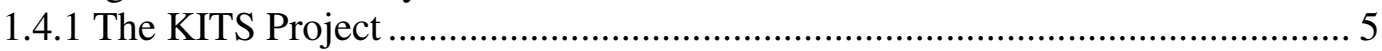

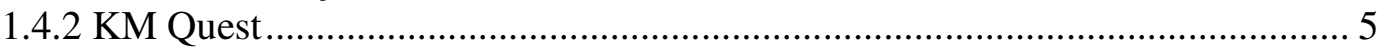

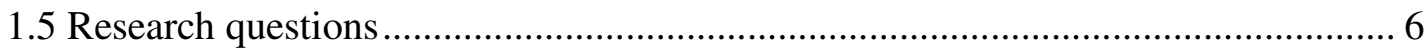

\section{Chapter 2}

Developing a game simulation model for the knowledge management game...........9

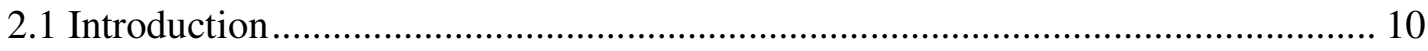

2.2 Limiting the scope of the model ................................................................... 11

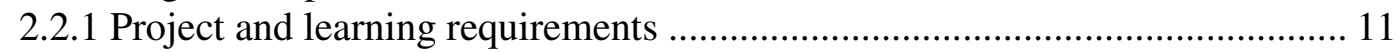

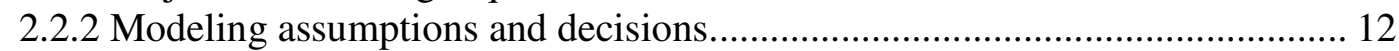

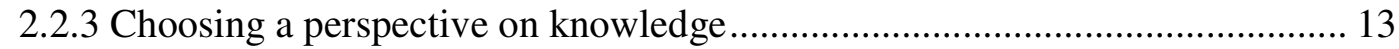

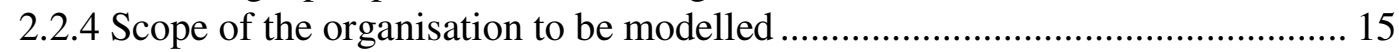

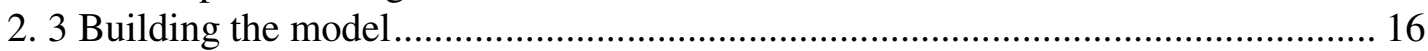

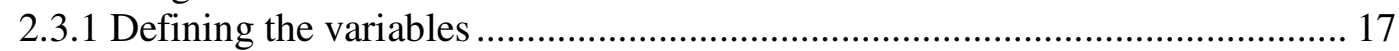

2.3.2 Defining the relations between variables ...................................................... 24

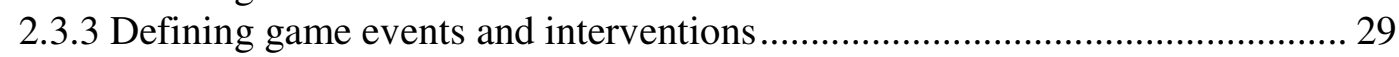

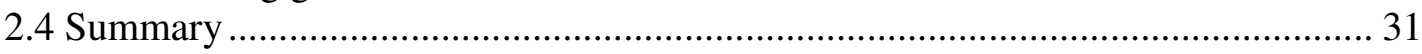

\section{Chapter 3}

Translating the model into a computer program...................................33

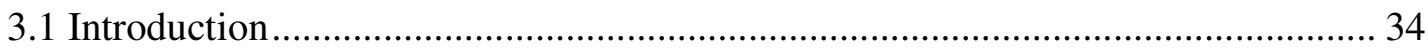

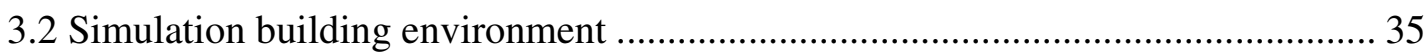

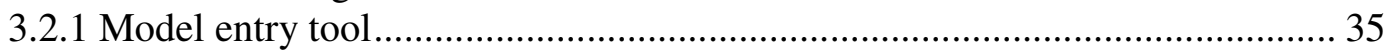

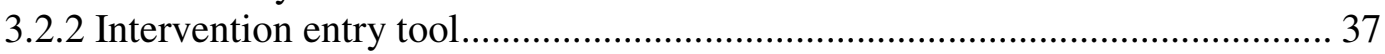

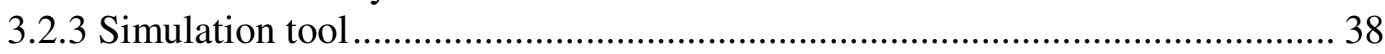

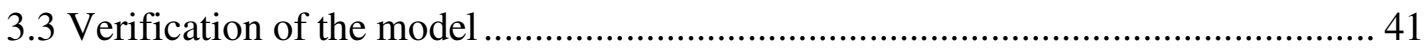

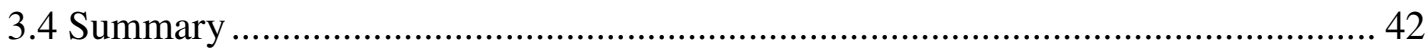

\section{Chapter 4}

Validation of the game simulation model - design of the study ......................43

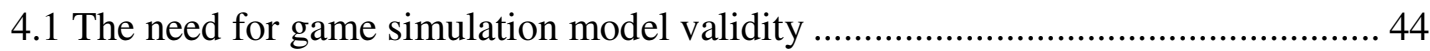

4.2 Literature review and practical implications...................................................... 44 


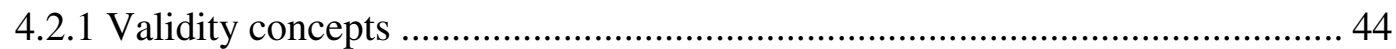

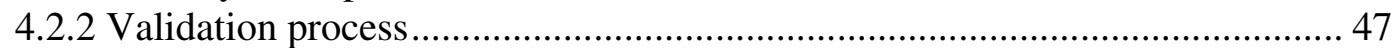

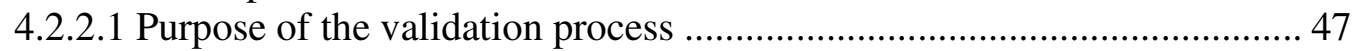

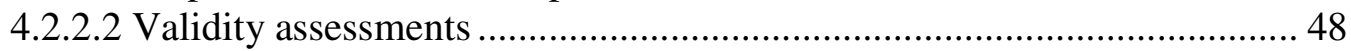

4.3 Concepts of the KM Quest game simulation model validity ............................... 53

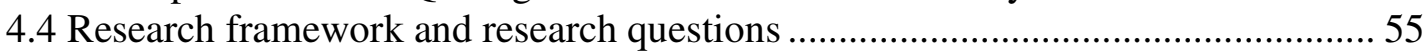

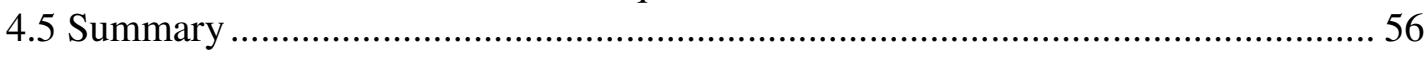

\section{Chapter 5}

The model's fidelity: investigating validity $. . . \ldots \ldots \ldots \ldots \ldots \ldots \ldots \ldots \ldots \ldots \ldots \ldots \ldots \ldots \ldots \ldots . . . .57$

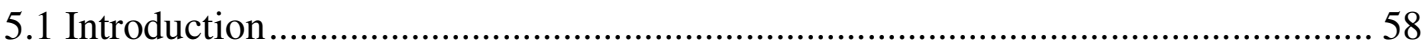

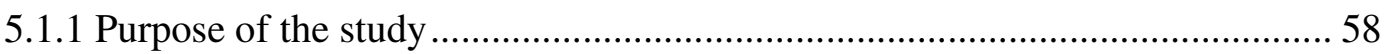

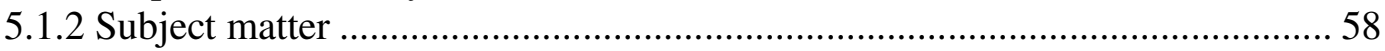

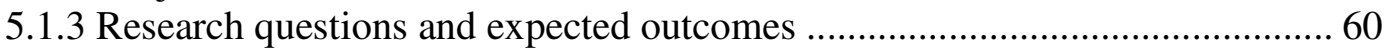

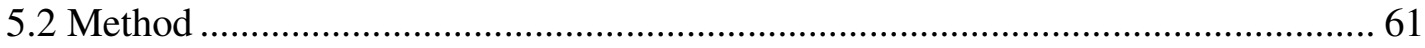

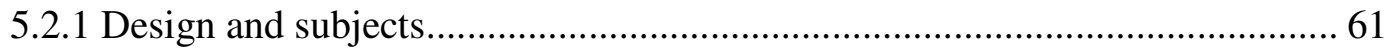

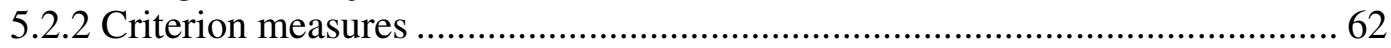

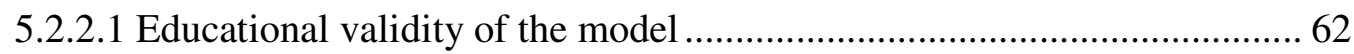

5.2.2.2 Perceived representational validity of the model ..................................... 65

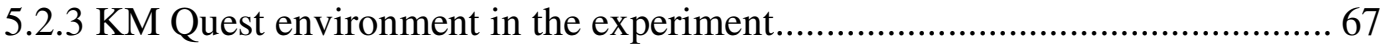

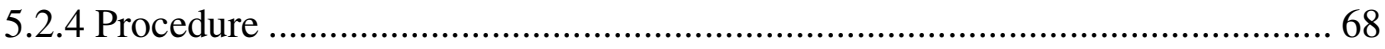

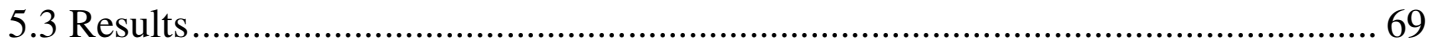

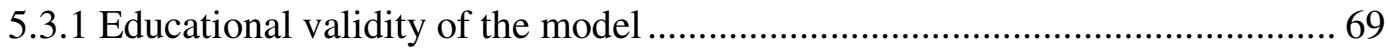

5.3.2 Perceived representational validity of the model............................................. 71

5.3.3 Relationships between perceived representational validity and educational

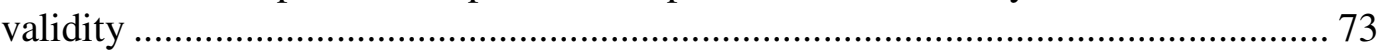

5.3.3.1 Relationships between performance indicators in the game (internal educational validity) and perceived representational validity ........................... 74

5.3.3.2 Relationships between perceived representational validity and educational

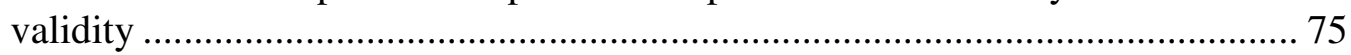

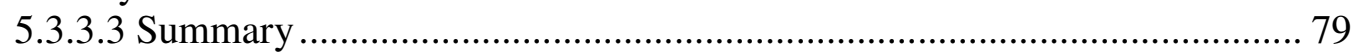

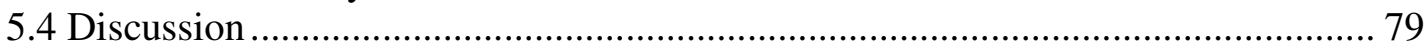

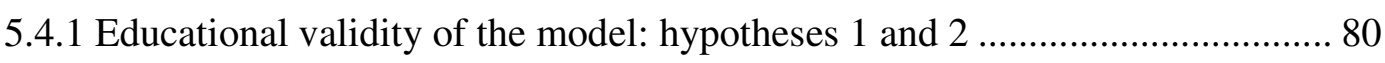

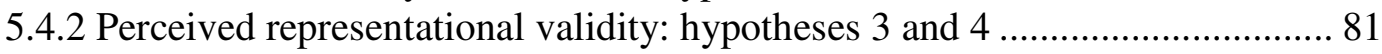

5.4.3 Relationships between the educational validity of the model, its fidelity and perceived representational validity .................................................................... 81

\section{Chapter 6}

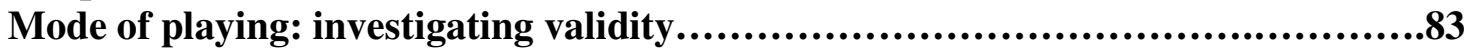

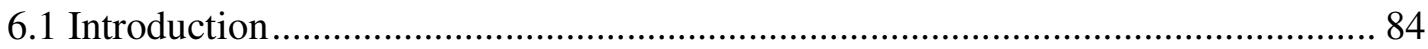

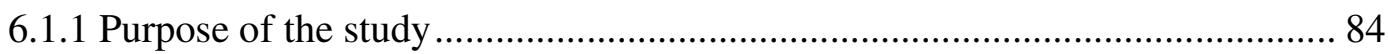

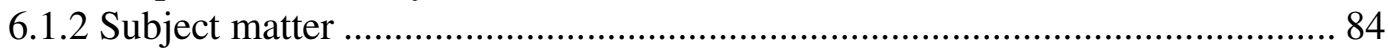

6.1.3 The KM Quest simulation model and its support in the experiment ............... 86

6.1.4 Research questions and expected outcomes ............................................... 88 


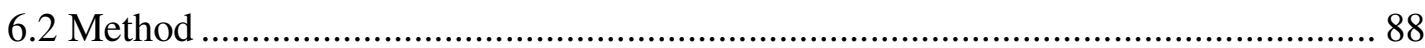

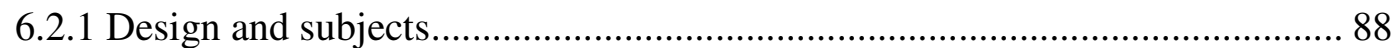

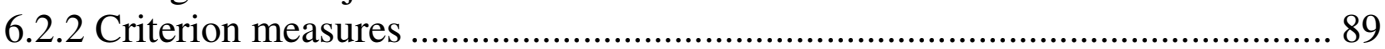

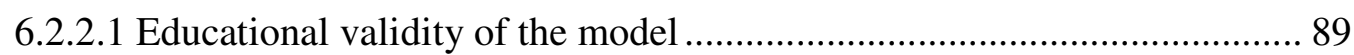

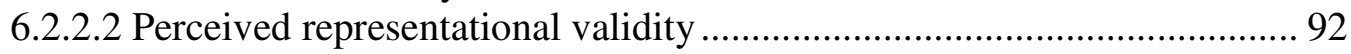

6.2.2.3 The KM Quest environment in the experiment .................................... 93

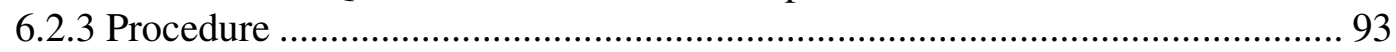

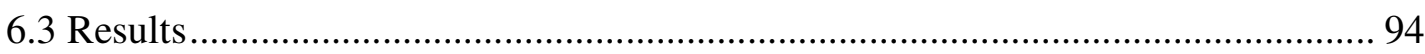

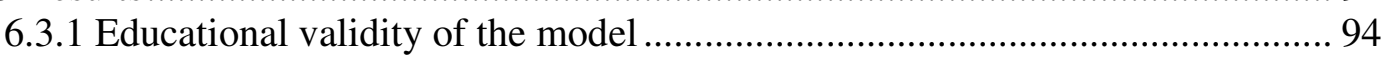

6.3.2 Perceived representational validity of the model......................................... 96

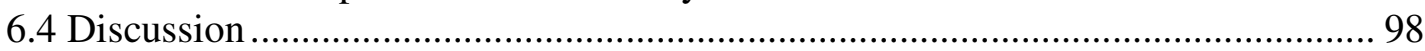

\section{Chapter 7}

Exploring representational validity..$\ldots \ldots \ldots \ldots \ldots \ldots \ldots \ldots \ldots \ldots \ldots \ldots \ldots \ldots \ldots \ldots \ldots \ldots \ldots 1$

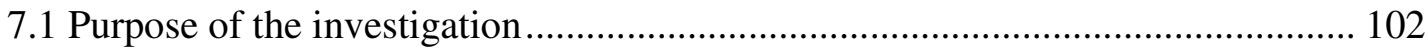

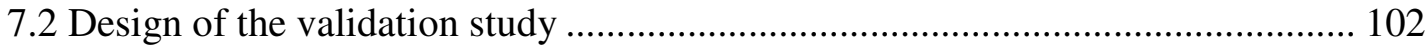

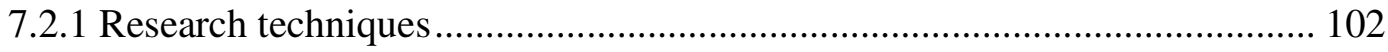

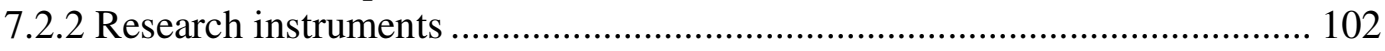

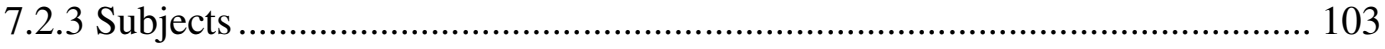

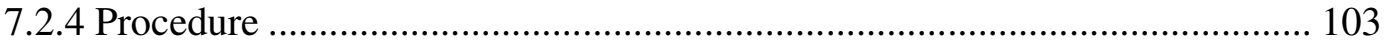

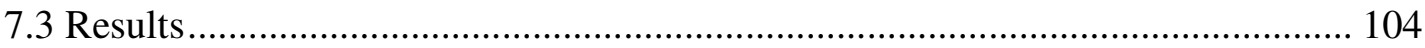

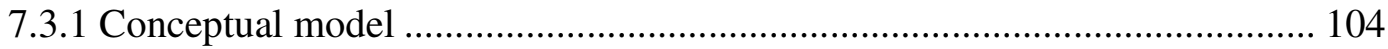

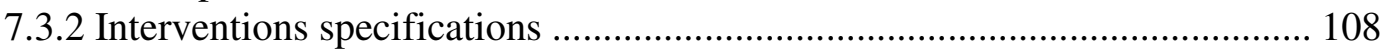

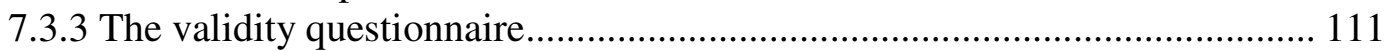

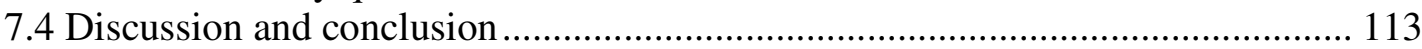

\section{Chapter 8}

Across the studies.............................................................115

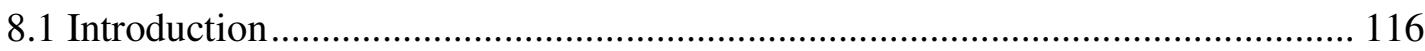

8.2 Comparison between students' game results and students' validity judgments ... 117

8.2.1 Differences in the model representation and the game environment............. 117

8.2.2 Results of the cross-experimental analysis ................................................. 118

8.2.2.1 Internal educational validity: game performance ................................ 118

8.2.2.2 Perceived representational validity ................................................ 120

8.3 Comparing student's and expert's game results and validity judgments.............. 124

8.3.1 A special case of educational validity ..................................................... 124

8.3.2 Perceived representational validity and representational validity ................. 124

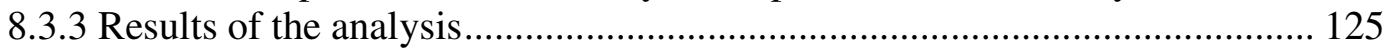

8.3.3.1 Educational validity of the model .................................................... 125

8.3.3.2 Perceived representational validity and representational validity of the

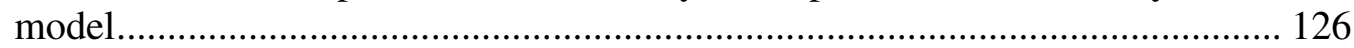

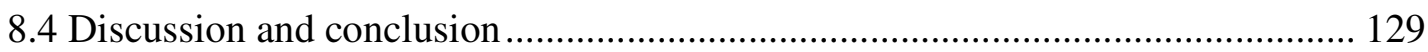




\section{Chapter 9}

General Discussion

9.1 Summary 132

9.2 To what extent does the model reflect actual phenomena? 137

9.3 Does the model support and provide learning about the phenomenon? ............... 138

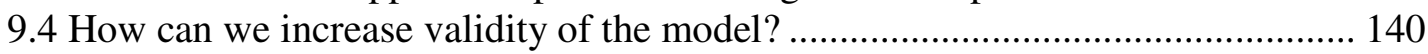

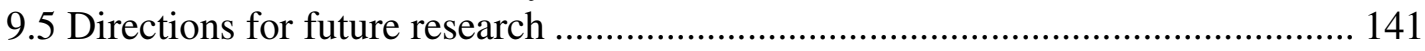

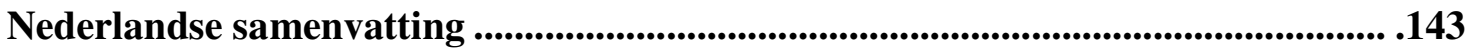

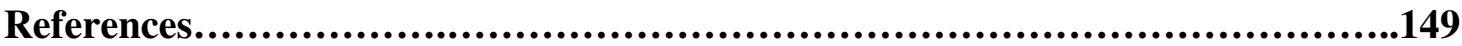

Appendices......................................................................157

Appendix 1. Final set of variables accessible for the players .................................... 158

Appendix 2. Variables and interventions/events for the R\&D domain ...................... 160

Appendix 3. Example of the specification of interventions...................................... 161

Appendix 4. Example of the specification of events .................................................. 162

Appendix 5. Post test item measuring conceptual knowledge.................................... 163

Appendix 6. Post test item measuring strategic knowledge ..................................... 164

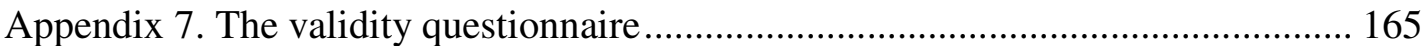

Appendix 8. The modeling questionnaire …............................................................. 169 


\section{Chapter 1}

\section{What is this book about?}

In this introductory chapter the key elements of the study are presented, including theoretical foundations to be considered, research problems to be investigated, and an outline of the structure and content of the thesis. 


\subsection{Introduction}

For many years practitioners and researchers have been discussing a knowledge-driven economy, knowledge-intensive and learning companies, knowledge workers and knowledge productivity, knowledge-based services and the value of knowledge (Sveiby, 1997; Edvinsson \& Malone, 1997; Davenport \& Prusak, 1998; Boisot, 1999; Tiwana, 2000 ; Buckman , 2004; Dalkir, 2005).

Researchers and practitioners generally agree that knowledge management $(\mathrm{KM})$ is not a goal in itself, but a strategy, policy or process which should bring a company additional value in monetary or absolute terms of returns on investment, or in qualitative terms such as fostering better decision making processes, which create customer value or achieve the company's objectives. In other words, they emphasize that there is a dependency between the knowledge household of a company and the company's business performance. Despite many discussions concerning the contribution of KM activities to business performance, only "few if any companies have thus far been able to establish a causal link between their knowledge management activities and their business performance, regardless of how it is measured" (Davenport, 1999, p. 2-8). Thus the problems of establishing a causal link, tying knowledge to organizational performance and to representing the link in a formal (mathematical) model, are still present and challenging. Moreover, there are no models, in theory or practice, which demonstrate how dynamic changes in the knowledge household of a company relate to dynamic changes in business indicators of that company. This book is about building and evaluating such a model. Specifically, this book will review the building of a game simulation model for a knowledge management game to teach and learn KM knowledge and skills and evaluate this model from different perspectives.

The process of model building begins and runs in parallel with the theory development process. The terms 'theory' and 'models' are often used interchangeably in the theory development literature and research (e.g. Dubin, 1978), models are seen as minitheories (Herskovitz, 1991) or theories "in which all of the components are represented by symbols which can be manipulated according to the provisions of a welldefined formal discipline, typically a branch of logic or mathematics" (Stanislaw, 1986, p.174) or models are seen as an output of a theory building process (Carlile \& Christensen, 2005). Another point of view (Bunge, 1998, p. 439) is that "theories deal with [...] models that are supposed to represent, in a more or less symbolic way and to some approximation, certain aspects of real systems". Adopting these ideas, we can say that we intend to build a model which represents a real-world phenomenon and can be used in the knowledge management domain just as many other different models and at the same we contribute to knowledge management theory by providing ideas about how to relate knowledge processes in a company with the company's business performance and outcomes.

Models are built for specific purposes. The purpose of the knowledge management game simulation model is to help players of the game to learn about relationships between knowledge management processes in organizations and their business achievements, to show how changes in the knowledge household of a company influence the company's "hard" outcomes, and to teach decision making and problem solving skills in the knowledge management domain. In the KM domain, problems are multi-faceted, 
complex and without univocal outcomes (Wiig, de Hoog \& van der Spek, 1997). In this area the acquisition of decision-making and problem-solving knowledge and skills appears to be a difficult task (Leemkuil, de Jong, de Hoog, Cristoph, 2003). Nurmi and Lainema (2003) pointed out that the biggest challenges in business education are difficulties in applying theoretical subject knowledge in real life settings, inabilities to handle complex and ill-defined problems, and the lack of a consistent and holistic conception of business processes. This raises the question of how one can provide students or novice managers with the opportunity to think like professionals and to develop problem-solving skills. Solutions to this problem can be found in experiential learning-learning by games and simulations. These techniques give a greater insight into the problem, allowing one to improve abilities to deal with multiple realities and look for solutions to complex problems without destroying their variety and to test alternative courses of action (Klabbers, 1989; Szymankiewicz, McDonald, \& Turner, 1988). Although, the ideas about learning with game and simulation environments are the same, game environments, in comparison with simulation environments, provide "a conceptual framework useful for summarizing and communicating a set of important interrelationships", rather than precise or imprecise projections or a "philosophical exploration of the logical consequences of a set of assumptions without any necessary regard for the real-world accuracy or usefulness of the assumptions" (Meadows, 2001, p. 525). Additionally, people tend to believe that simulation models are true. Meadows (2001, p. 523) noticed that users "passively accepted [...] scenarios as predictions rather than interpreting them as illustrations of alternative possibilities for taking actions". Therefore, our approach was to provide a conceptual frame-an environment in which participants can become immersed in the problem and test interrelationships between the knowledge of a company and the company's performance. In the case of simulating a difficult to observe reality, we have to be sure that the game simulation model produces plausible behavior to teach the right interrelationships rather then evoking erroneous assumptions. As Peters, Visser and Heijne (1998, p. 20) emphasize, "if we want to make inferences about reality based on experiences and knowledge acquired in the game, we have to be sure that the game model is a good, or valid, representation of the real situation". In other words, errors in the game simulation model could lead to implausible experiences thereby causing players of the game to construct incorrect mental models of the investigated phenomenon (Peters, Visser \& Heijne, 1998; Sterman, 2002; Feinstein \& Cannon, 2002). Therefore, evaluating the game simulation model by assessing its validity is a crucial aspect.

\subsection{Evaluation of the model}

The evaluation of simulations and games is a challenging and difficult task. One of the main problems with simulations is how to "evaluate the training effectiveness [of a simulation]" (Hays \& Singer, 1989, p. 193). Feinstein and Cannon (2002) pointed out that only a few studies claim that the benefits of simulations are supported with substantial research. They argued that the problem lies in the inconsistency of concepts, terms and purposes of evaluation across the studies, therefore making it difficult to build a coherent program of validation research. For example, practitioners and researchers in the field of instructional design and gaming compare games' effectiveness with traditional teaching 
methods (Randel, Morris, Wetzel, \& Whitehill, 1992; Jacques, 1995; Wolfe, 1985, 1997; Herz \& Merz, 1998). Game designers often utilize summative and formative evaluation (Kriz \& Hense, 2006) while simulation designers aim to validate their simulations (Law \& Kelton, 2000; Balci, 1998). In many studies terms like 'evaluation' and 'validation', and 'game' and 'game model' are used interchangeably (Peters, Vissers \& Heijne 1998; Wolfe \& Jackson, 1989). In our view, this confusion simply arises from the fact that in a game evaluation study a game simulation model cannot be extracted from the 'body'the game. From another point of view, the game loses its functionality without the underlying model. To avoid any confusion for the reader, we stress that this research is focused on the evaluation and validation of the game simulation model.

Another dilemma in this field is that games and simulations are designed for specific purposes. Such function-oriented design of the games and simulations creates situations in which the same simulation "might receive a very positive evaluation as a learning tool, but it might fare quite poorly as a tool for modeling actual real-world phenomena" (Feinstein \& Cannon, 2002, p.437). At the same time as argued by Größler (2001, p.72) "the absolute efficacy of [...] simulation tools cannot be answered generally" and "comparisons with other teaching methods are not fruitful. The only evaluation approach open [...] is testing of business simulations which are systematically varied in one feature". Following this idea can we find out which features of the game simulation model provide a better representation and a better understanding of a phenomenon? Can we find an optimal solution?

In the research reported in this thesis, we will seek to validate the game simulation model from two perspectives (the model as a replication and representation of a realworld phenomenon and the model as a tool which supports learning knowledge management skills), and we will also investigate how we can optimise the model's validity.

\subsection{Thesis outline}

This research is based on theoretical and practical issues from several domains:

- Knowledge management supplies us with the object of the study and the theories and models.

- Simulation and gaming domains provide us with the guidelines to build the game simulation model and with the validation methods.

- Instructional design supports us with methods to evaluate learning and to assess the educational validity of the game simulation model.

Since our objective is to build a game simulation model, this study can primarily be categorized as a model development and evaluation process or as a simulation development and evaluation process.

According to Stanislaw (1986), a simulation development process consists of three phases: building a theory, constructing the model and translating the model into a computer program. Law and Kelton (2000) in their life-cycle of simulation, add two more steps which are dealing with simulation validation and credibility.

We arranged the further chapters in this book in accordance with these phases of the simulation development process. Thus, chapter 2 discusses the process of building a theory and the development of the game simulation model for the knowledge 
management game. In this chapter we present several approaches to formalize knowledge and our modeling assumptions, describe requirements for the model, which were taken into account, and finally explain and present the game simulation model. Chapter 3 focuses on the translation of the model into an executable simulation in such a way that allows tracing and fixing of modeling and programming errors. Chapter 4 discusses the validity aspects of the game simulation model and narrows our research questions. Chapters 5, 6, and 7 reflect validity aspects of the game simulation model. In these chapters different studies are presented which were conducted to investigate the validity of the game simulation model. Chapter 8 combines and discusses the results of the experimental studies. Chapter 9 provides a summary of the work and outlines possibilities for the further development of our theory and the improvement of the game simulation model.

\subsection{Background of the study}

\subsubsection{The KITS Project}

The development of the knowledge management game simulation model was partly done within the Knowledge management Interactive Training System (KITS) project $^{1}$ which lasted from May 2000 until January 2003. The aim of the KITS project was to build an internet-based collaborative learning environment to improve the training of people working in the emerging field of knowledge management. This goal resulted in the training system embodied in a collaborative internet-based simulation game: KM Quest (Leemkuil, de Jong, de Hoog, Cristoph, 2003). The first and second prototypes of the model were developed during the project and two evaluation studies were carried out. Based on data and findings from these experiments, the model was adjusted and several experiments were conducted in order to explore the validity of the game simulation model.

\subsubsection{KM Quest}

A prototype for the KM Quest learning environment was a knowledge management business game, which was developed in 1997 and played at CIBIT consultants/educators-a Dutch consultancy company. In this game, teams of players had to manage a fictitious company called Coltec and react to unexpected events related to the company description provided (De Hoog, Van Heijst, Van der Spek, Edwards, Mallis, Van der Meij, and Taylor, 1999). A weak point of that game was that "the actions taken do not really change the state of the world as an input for the next cycle of the game" (De Hoog, et al. 1999, p.10-4). These authors suggested that "a strong

\footnotetext{
${ }^{1}$ Work partially supported by European Community under the Information Society Technology (IST) RTD programme, contract IST-1999-13078 (KITS). The author is solely responsible for the content of this article. It does not represent the opinion of the European Community, and the European Community is not responsible for any use that might be made of data appearing herein. Partners in the KITS project are University of Twente (NL), University of Amsterdam (NL), CIBIT (NL), ECLO (UK), Tecnopolis (I) and $\operatorname{EADS}(F)$.
} 
(computational) model is required of the way in which KM actions affect the knowledge household of an organization" (De Hoog, et al. 1999, p.10-4) The experience gained from playing the Coltec game and the practical KM experience of CIBIT grounded the development of the game simulation model for the KM Quest game.

The game simulation model enriches the learning environment with a feedback mechanism and consists of the following components:

- A case description with general information about the company,

- The core of the game simulation model-a set of business performance variables, whose values change dynamically with time and depend on actions taken by players,

- A set of external and internal events, influencing or not influencing the simulated company,

- A set of actions or interventions that players can do in order to react to events or changes in business performance variables.

As a result, in the KM Quest learning environment, players have to manage a fictitious company called Coltec over three years. Each year is divided into four quarters (or game periods) by invoking available KM interventions from a predetermined set in order to react or respond to events and the state of the business performance variables. Each period the new situation is simulated based on previously chosen interventions, events and the time frame. This feedback is the new situation for the next game period. The game can be played individually on a stand-alone PC or collaboratively in teams of up to four players over the Internet.

Development of the KM Quest learning environment provided the project team with many research problems and opportunities. Colleagues who were involved in the KITS project that resulted in the development of the KM Quest learning environment investigated different aspects of the KM Quest learning environment: learner support in the game by means of different instructional support tools (Leemkuil, 2006), the role of metacognition and its support in the game by a normative KM model (Christoph, 2006), and learner support by means of visualization (Purbojo, 2005). This thesis, apart from building the game model and evaluating the model, also explores the aspect of learner support from the perspective of the game simulation model.

\subsection{Research questions}

The primary goal of the research described in this thesis was to build a game simulation model that represents interrelationships between knowledge and business performance in a company, promotes understanding of this phenomenon for players of the game and promotes the learning and development of decision-making skills in the knowledge management domain. The second goal we pursued in this research was investigating the validity of the game simulation model, which in our view influences the ability of the model to support and promote learning about the phenomenon. Different features of the model (for example, different visualization of variables (Purbojo, 2005) and the transparency of a model versus a black-box approach (Machuca, 2000; Größler, 1998; Größler, Maier \& Milling, 2000), could have an influence on the ability of the model to support learning. In this thesis we investigate how we can strengthen the instructional 
power of the model by providing or closing access to the knowledge process related variables, thus, varying fidelity of the model and by changing the mode of playing.

Research on the validity aspects of the game simulation model for the knowledge management game has to find answers on the following questions:

- To what extent does the model reflect actual phenomena?

- Does the model support and provide learning about these phenomena?

- How can we increase validity of the model?

These research questions will be more specifically addressed in chapters 5, 6, 7, and 8 . 

Chapter 2

\section{Developing a game simulation model for the knowledge management game}

This chapter begins with the purposes of building the game simulation model and an explanation of the requirements on the model. Next, several approaches to measure knowledge and its effects are reviewed. The modelling assumptions that were used to build the model, which can represent the influence of knowledge management activities on the overall organizational performance, are discussed. The chapter ends with an explanation of the model and a further discussion of research goals. 


\subsection{Introduction}

Currently there is no accepted and well-established theory of measuring and formalising knowledge, therefore our work can be seen as a significant theoretical contribution to this area. All known knowledge management models which measure knowledge tend to reflect the results of knowledge development (education of employees, years in the profession) or the results of knowledge use (number of patents, number of contracts and so on). Moreover, there are no models, in theory nor in practice, which demonstrate how changes in the knowledge household of a company influence company performance or, in other words, demonstrate "behaviour" of knowledge and relate it to dynamic changes of other company indicators. Thus, in order to better understand this problem, we need first to build a theory about the relation between knowledge in a company and company performance and then build a model based on this theory.

However, a theory about the relation(s) between a company's knowledge household and organisational outcomes cannot be easily proven in reality. Therefore, a solution to this problem can be to simulate reality. A company and its activities can be represented in the form of a simulation and its performance and outcomes could be observed over the simulation runs by potential users in order to obtain judgments about the simulated phenomenon. These judgments can be used to estimate the plausibility of the theory as it is embodied in the simulation. In other words, simulation can be used as a method to specify and test theories.

Developing a simulation generally entails several phases (Stanislaw, 1986). The researcher begins by building a theory to account for the real-world behavior or phenomenon that is being addressed. This theory may be simply a collection of statements that are explanatory in nature. The statements need not necessarily be accurate in the sense of representing "truth", but they must specify causal relationships. During the step of building a theory a modeler is driven by three main principles: reduction, abstraction, and symbolization (Stanislaw, 1986; Peters, Vissers \& Heijne, 1998). Reduction entails that a designer makes a selection of elements from the modeled system that have to be included in the game model: he or she includes the elements that seem relevant and important, and leaves out the elements that are less important. The second principle - abstraction - implies that the elements included in the game model are not necessarily as detailed as they are in reality: the designer deliberately simplifies them to make the model less complex. The last principle - symbolization - deals with the fact that the elements and relations of the modeled (reference) system are modeled into a new symbolic structure, namely, into scenario, roles, rules, and symbols, which are representing the most important elements of a game. During this process several errors can be made (Peters, Vissers \& Heijne, 1998; Irvine, Levary \& McCoy, 1998):

- The designer fails to take full account of the objectives of the game.

- The designer lacks a thorough knowledge of the modeled system - he or she is not capable of estimating the relative importance of the elements of the reference system correctly.

- The designer may be guided by the opportunities and/or the restrictions of the game instead of by the features of the modeled system and the main objectives of the game. 
To avoid these errors, the task of building a game simulation model for the knowledge management game, in our view, has to cover the following aspects:

- Requirements should emanate from the project and the learning objectives of the game

- Decisions about the overall nature of the model should be made in terms of different types of simulation options

- Scoping of the model should be made from a knowledge (management) perspective

- Scoping of the model should be made from the viewpoint of organisational types

- The actual design of the model should be based on these modelling decisions This chapter elaborates on these aspects in the sections below.

\subsection{Limiting the scope of the model}

Theoretically, anything can be modelled, but there is no unified guide to modelling. Each modelling study is unique, and this uniqueness comes from the modelling purposes and/or modelled object or phenomena. One would agree that building a model should be rational and rely on some purpose. As stated by Sterman (1988, p. 211) "A model must have a clear purpose, and that purpose should be to solve a particular problem. A clear purpose is the single most important ingredient for a successful modelling study". Therefore, the purpose of a game simulation model for the knowledge management game should be narrowed and refined in line with several aspects which we address in this section.

\subsubsection{Project and learning requirements}

The rationale for developing the KITS learning environment was derived from user feedback gained from participants of KM courses who played a knowledge management game developed in 1997, based on a fictitious company called Coltec (see 1.4.2). In the KITS project (Leemkuil, de Jong \& Ootes, 2000; Haldane, 2000) developers decided to enhance features of the game and create a new internet-based collaborative learning environment - a new game. From the learning objectives of the environment and project requirements for the game, we focus only on those which are important from the point of developing the knowledge management game simulation model. The relevant game simulation model development learning objectives are:

- Learning in the KITS environment should lead to implicit, intuitive knowledge about the content of KM actions and their consequences.

- Learning in the KITS environment should lead to explicit knowledge about certain aspects of the domain.

Specifically the game requirements included the following (Leemkuil, de Jong \& Ootes, 2000):

- The game should include challenging goals for the learners. These goals are "real life" goals and concern outcomes of the simulation model affecting variables that signify a certain business outcome. 
- The game should include the idea of coupling resources to certain actions. Learners should always have to make a trade-off between the effects of their actions and the costs involved.

- The game should include realistic cases that lead to a sense of involvement on the side of the learner.

- The game should have the possibility to generate random or unexpected events.

These learning objectives and the game requirements suggest that the model should support the effectuation of knowledge management activities and interventions in the game in a systematic way and propagate their effects on the company. This could be achieved with an implementation of the model having the following features and characteristics:

- A company should be described by means of a formal mathematical model in which the company is represented by commonly used indicators and knowledge variables and the relations between them.

- There should be connections between knowledge variables and regular business variables.

- There should be a connection between players' actions-interventions, events and the model variables.

- There should be a propagation of effects of players' actions or events on the indicators.

- The model should exhibit dynamic behaviour.

These prerequisites were taken as starting points for the model development process. Nonetheless, some theory about modeling was needed to see how designers and modelers implement their ideas into workable and executable models.

\subsubsection{Modeling assumptions and decisions}

Any process or object can be modelled and represented as specifically related parts: subprocesses or sub-objects. The facility or process to be modelled is usually called a system. In order to study how the system works, modellers make a set of assumptions. These assumptions, made in the form of logical or mathematical relationships, constitute a model. Thus, a model is a representation of an actual system (Banks, 1998), which is made by a modeller. There are many types of models. They can be classified as physical or mathematical, stochastic or deterministic, dynamic or static. Models that are executable by a computer are called simulation models.

There are three main dimensions along which simulation models are classified: static or dynamic, deterministic or stochastic, continuous or discrete (Law \& Kelton, 2000). A static simulation model is a representation of a system at a particular point in time, while a dynamic simulation model represents a system as it evolves over time. If a simulation model does not contain any probabilistic components, it is called deterministic. Discrete simulation concerns the modelling of a system that evolves over time by a representation in which the state variables change instantaneously at separate points in time. In the continuous simulation models the state variables change continuously with respect to time. The kind of model we build and the features it has, 
depends on the nature of the modelled phenomenon and modelling assumptions made to achieve the goals of the modelling study.

The knowledge management game simulation model has to support an understanding of the importance of knowledge and KM activities for a company by simulating relationships between knowledge properties, activities of decision makers and organisational outcomes.

Before deciding on the nature of the model, it should be clear what modelling assumptions are made to satisfy the modelling purpose. The theoretical assumptions for the case of the knowledge management game are:

- Knowledge is a quantifiable object that can be measured using relative scales (intermediate measures).

- Performance of any business unit depends on the quality of knowledge and efficiency of knowledge usage or utilisation. Consequently, the business results also depend on the knowledge and the utilisation of it. The ideal situation for a company is to have highly knowledgeable employees and an effective organisation of work processes, that is, an effective application of knowledge.

- Knowledge naturally depreciates due to ageing and volatility. If there is no increase and renewal of knowledge in a company, performance declines over time.

- Changes from outside or inside a company influence its organisational 'knowledge household'.

In real life managers obtain financial and other information about a company over certain periods of time, while the behaviour of the system (the company) is continuous. Thus, the model we build should be dynamic and exhibit continuous behaviour. However, since in the game players must deal with specific events which affect the company, the game simulation model should have also a discrete-event character. Natural depreciation of knowledge can be modelled by giving a decay function to variables which describe knowledge. As a result, our game simulation model should be a dynamic model and should exhibit discrete-event-continuous behaviour.

These modelling requirements and theoretical assumptions contributed to the modelling process. In addition, to be able to build the model, create variables and draw relationships and inferences between them, we have to take into account the domain and its specific characteristics.

\subsubsection{Choosing a perspective on knowledge}

The idea of knowledge bringing a competitive advantage is a common long-held notion. "In an economy where the only certainty is uncertainty, the one sure source of lasting competitive advantage is knowledge" (Nonaka, 1998, p. 175). Knowledge is an asset or a resource that can bring the company valuable benefits and a leading position in the field but only if it is properly managed. At a gross level of analysis, we can say that knowledge management initiatives and organisational outcomes positively co-vary. "Successful knowledge management efforts eventually improve financial performance by increasing sales, decreasing expenses, or both, while unsuccessful knowledge management efforts increase expenses more than they increase sales" (Stone \& Warsono, 2003, p. 254). 
Although much effort has been put into measuring the impact of knowledge on organisational value, there are few methods formalising knowledge assets and linking them to organisational outcomes. In the early practice of measuring and evaluating knowledge even advanced techniques such as The Intangible Assets Monitor (Sveiby, 1997), The Balanced Score-Card (Kaplan and Norton, 1996), The Skandia Business Navigator (Edvinsson and Malone, 1997), only linked and related knowledge to business outcomes by presenting results of knowledge usage (e.g., number of patents, number of contacts with customers) or personal properties (e.g., years in the profession, educational degree, etc). Tiwana (2000) calls them "proxy measures". They "surely do a better job at approximating gains emerging from effective handling of knowledge, but they still underestimate the actual gain as they measure "knowledge stock" and not "knowledge flows" (Tiwana, 2000, p.163). Later models, such as Value Chain Scoreboard (Lev, 2001) and Financial Method of Intangible Assets Measurement - FiMIAM (Rodov and Leliaert, 2002) built on the advances of the earlier models; they consider value-creating activities and link components of intellectual capital to the market value of a company. Nevertheless, they are still 'black-box' approaches, because "managers are no better off understanding exactly what are the company's intangible resources or their specific contribution" (Bontis, 2001, p.55). There is yet no clear idea why and how knowledge and knowledge processes relate and contribute to business performance. Nevertheless, "once we recognize the importance of a concept, we can almost always find ways to measure it" (Sterman, 2002, p.524).

The possible solution, according to Davenport (1999), can be found in developing intermediate measures to relate knowledge management to organisational performance. Taking this statement as a problem, we can aim in our research to develop a model, which:

- Dynamically relates knowledge to organisational performance.

- Represents static and dynamic characteristics of knowledge - "knowledge stocks and knowledge flows".

Thus, the problem to solve and the job to be done are to determine how a company has to be modelled and to formally represent its knowledge "stocks and flows". We are examining knowledge from the knowledge production perspective (Holsapple, 2003, p.168) which means that:

- Knowledge stock is an inventory of knowledge available to one or more processors or agents. The way knowledge is represented in a particular stock could include any of the representational modes (symbolic, mental, behavioral, digital, etc.)

- There are two main kinds of flows: knowledge transfer from one stock to another and knowledge flow from a stock into itself.

Another definition of knowledge flow is given by Newman (2003), who believes that knowledge flows are constituted by three concepts: agents, artefacts, and transformations. He defines them as "sequences of transformations performed by agents on knowledge artefacts in support of specific actions or decisions" (p. 304). From his point of view, transformations are the behaviours that agents perform on artefacts. The list of all possible behaviours is too large and these behaviours are grouped in several categories of activities, which we call knowledge processes. Adopting ideas from these two authors, our idea of a formal representation of knowledge can be narrowed and seen 
as a representation of knowledge stocks by concepts that are close to the "amount" of knowledge in a specific domain and the representation of knowledge flows by the concept of knowledge processes.

\subsubsection{Scope of the organisation to be modelled}

As there are innumerable dimensions among which one can characterise or classify a company (e.g., size, industry, strategic focus, market orientation), it is not feasible to create a unified model that covers every organisation; therefore, a decision has to be made concerning the type of organisation to be modelled. Additionally, we have to model a company from a knowledge perspective, which is related to issues of strategic development rather than tangible issues such as size, market niche and industry. This idea also corresponds with what we have already said about existing knowledge measurement models, that is, looking at a company from the value-creation perspective rather then from a value-capturing perspective.

Among other approaches (e.g., Mintzberg's (1983) classification, which is based on the structure of the company), several authors provide a rationale for classifying companies based on their strategic focus. Treacy and Wiersema (1995) proposed a classification, though not ideal, that gives criteria by which organisations can be defined at an abstract level and from the perspective of a value proposition. They distinguish three main strategic directions for a company, which can be used to identify the type of a company, and consequently to focus on crucial knowledge areas (domains) for these companies:

- Operational excellence: a company competes mainly in terms of costs. It tries to streamline processes in such a way that, while keeping the required quality, the costs of operating are minimised.

- Product leadership: a company competes by rapidly bringing innovative products to the market. This implies short product life cycles and innovative research and development.

- Customer intimacy: a company competes by trying to become a partner with a limited number of other companies by providing customised solutions.

Wiig (1995) mentions strategic factors that lead to superior performance such as organizational creativity, operational effectiveness, and the quality of products and services. He emphasised that these factors are improved when better knowledge is made available and used competently. The same three general categories: customer intimacy, product-to-market excellence, and operational excellence are considered by O'Dell, Elliot and Hubert (2003) in their description of key knowledge components. They characterised these company types as a value proposition or, in other words, as a business rationale for a company to embark on an initiative or institute a process.

This classification is particularly helpful in our case, since it allows the modeller to model knowledge areas that are crucial for the company, leaving the structural views on the company outside the scope of the model. Modelling a company according its structure poses two difficulties: 1) we have to distinguish and model the same knowledge on different organisational levels that can overload the model and brings us to a too specific level (e.g., the individual employee); and 2) we have to narrow down the applicability of 
the model to those specific companies sharing a similar structure, while the knowledge gained from the model's use (learning about knowledge management) should be generalised or transferred to a broad range of companies which do not have that particular structure. Another way to model a company is to model in specific detail its business processes and/or knowledge processes. However, this would bring us to the operational level, and change the game purpose from the optimisation of the knowledge household as a comprehensive company resource to the optimisation of specific business/knowledge processes.

For the knowledge management game simulation model, a product leadership type of company was chosen to be modelled. The reasons for this choice are:

- The focus can be on the result of the business processes and the result of the application of knowledge rather than the exact way the products were made and developed.

- There is no need to handle complicated production processes or customer relationships, rather one can focus on products as entities and abstract processes to generate these products; Players of the game can generalise and potentially transfer model relationships to their own companies or experiences.

From this point of view, knowledge and knowledge processes can be modelled in general without detailed specification of agents, artefacts and transformations and they should be related to business results. Referring to the idea of knowledge 'stocks' and 'flows' the underlying model should represent for the players of the game dynamical changes in the business indicators based on the changes of knowledge 'stocks' and knowledge 'flows' of the company (Figure 2.1).

Figure 2.1 Relationships between knowledge and organisational outcomes

\begin{tabular}{|l|l|}
\hline $\begin{array}{l}\text { Changes in } \\
\text { knowledge 'stocks' } \\
\text { and 'flows' }\end{array}$ & $\begin{array}{l}\text { Changes in business } \\
\text { indicators }\end{array}$ \\
\hline
\end{tabular}

How the knowledge 'stocks' and 'flows' are formalised and which variables are included in the model is described in the next section.

\section{3 Building the model}

While making a game simulation model and applying the principles of reduction, abstraction and symbolization (Stanislaw, 1986; Peters, Vissers \& Heijne, 1998), the designer of the model has to deal with such issues as simulation fidelity. Fidelity is defined as "the degree to which a model or simulation reproduces the state and behavior of a real world object or the perception of a real world object, feature, condition, or chosen standard in a measurable or perceivable manner; a measure of the realism of a model or simulation; faithfulness" (Gross, 1999). Feinstein and Cannon (2002, p. 426) define simulation fidelity as "the level of realism that a simulation presents to the learner". However, the notion of realism is confusing when talking about a knowledge 
management simulation model, as knowledge and knowledge processes are intangible assets. In contrast to tangible assets they cannot be seen and touched unless embodied in tangible objects like books (Reilly \& Schweihs, 1998; Berry, 2004). Nevertheless, on a higher abstraction level knowledge processes exist and they can be seen as a collection of small organizational processes which exist physically. For example, a knowledge sharing process might include processes like personal meetings, project group meetings, departmental meetings, conferences, and so on. To model knowledge processes at the physical level, we need to include in the model persons that participate in the meeting and the documents and information they discuss. This brings the model to the operational level which is too detailed for the learning purposes of the game. In the game we have to teach players to function as managers without challenging them with too much detail; Knowledge processes are abstract and might be similar for many companies, but operational processes from which knowledge processes are comprised are most of the time very specific to a company. For this we rely on the notion of functional fidelity given by Hays and Singer (1989, p. 50). Considering training situations, they defined fidelity as "the degree of similarity between the training situation and the operational situation which is simulated. It is a two dimensional measurement of this similarity in terms of: (1) the physical characteristics, for example visual, spatial, kinesthetic, etc.; and (2) the functional characteristics, for example the informational, stimulus, and response options of the training situation". Taking into account an old management proverb "You cannot manage what you cannot measure", we have to quantify knowledge and more abstract knowledge processes to teach the managing of these processes. Thus, by specifying and quantifying knowledge and knowledge processes in the game we decrease physical fidelity and to some extent the level of realism presented to the learners. At the same time we increase, in our view, the functional fidelity of the model and the game as it covers the key processes that matter when managing knowledge. To summarize: our modeling perspective refers to the functional aspects of an organization, not on it's operational aspects.

The question now is whether the specification and quantification of knowledge and knowledge processes will confuse players and limit their learning of how to manage knowledge processes or if it will have the opposite effect. Answering this question is an important part of this thesis.

\subsubsection{Defining the variables}

The first modelling step is to define the relevant variables which can be used to describe knowledge domains and knowledge processes, in other words, represent the knowledge stocks and knowledge flows contributing to the business level.

Examples of knowledge domains for these three mentioned types of companies and examples of business process variables can be found in Table 2.1 (Shostak, Anjewierden, de Hoog, 2002).

Based on the classification and the profile of product leadership companies, we define the following crucial knowledge domains that have to be modelled:

- Marketing and sales: these companies must commercialise their ideas quickly by reacting on the market changes and preferences and by quickly bringing their innovative products to the market. 
- Research and development $(R \& D)$ : they must be creative and originate new innovative products.

- Production: they must be able to overtake their own latest products and services and they must produce.

Table 2.1 Examples of knowledge domains and business processes variables

\begin{tabular}{|c|c|c|}
\hline Type of company & Knowledge domains & Examples of variables \\
\hline $\begin{array}{l}\text { Operational } \\
\text { excellence }\end{array}$ & $\begin{array}{l}\text { - } \text { Manufacturing } \\
\text { - Logistics } \\
\text { - Suppliers and purchasers } \\
\text { - } \text { Research and development }\end{array}$ & $\begin{array}{ll}\text { - } & \text { Equipment downtime } \\
\text { - } & \text { Operational costs } \\
\text { - } & \text { Time of production cycle }\end{array}$ \\
\hline $\begin{array}{l}\text { Product } \\
\text { leadership }\end{array}$ & $\begin{array}{ll}- & \text { Marketing } \\
- & \text { Research and development } \\
\text { - } & \text { Manufacturing } \\
\end{array}$ & $\begin{array}{l}\text { - Time of bringing a new } \\
\text { product to market } \\
\text { - Number of new patents }\end{array}$ \\
\hline $\begin{array}{l}\text { Customer } \\
\text { intimacy }\end{array}$ & $\begin{array}{ll}\text { - } & \text { Marketing } \\
\text { - } & \text { Services } \\
\text { - } & \text { Customer relation }\end{array}$ & $\begin{array}{l}\text { - Number of contacts with } \\
\text { the customers } \\
\text { - Number of services } \\
\text { - Number of new customers }\end{array}$ \\
\hline
\end{tabular}

There are many more knowledge domains in product leadership companies, and they can be further decomposed, but for the purposes of the game these domains are the most important from the point of the strategic focus of the company. More precisely, knowledge domains can be defined by using several techniques, such as basic knowledge analysis, knowledge mapping and others (Wiig, 1995), but in this case the company which is modelled could become too specific and inferences of the model could not be generalised to other companies. For example, for a company with three research labs we can decompose 'research' knowledge into specific knowledge residing in the three units instead of handling 'research' knowledge as a whole. The model becomes more specific with regard to one particular company and the knowledge to be modelled becomes also more specific as it would not make sense to decompose the knowledge over three labs without specifying the differences between them. If we consider 'research' knowledge as a 'whole' without decomposing it to the specific knowledge areas or organisational levels, the model can be applied to different companies operating in different areas. Research knowledge about chemicals differs from research knowledge about electronics. Modelling knowledge processes of a company with three research labs differs from modelling knowledge processes of another company with one lab, not only in terms of different structures, but also in terms of the knowledge household if different domain knowledge is applied in these labs. The purpose of the model is to introduce the phenomenon and to teach learners how to manage knowledge in general, not how to manage specific knowledge in one specific company.

After defining knowledge domains, the next crucial step is to define knowledge processes relevant for the company, since these processes represent knowledge flows and initiate contributions to knowledge stocks.

In the current knowledge management literature numerous examples of knowledge processes can be found. Some of these are presented in Table 2.2. 
In this table, the most frequently mentioned knowledge processes are knowledge creation, transfer, utilization, and retention. For the purposes of the game it is important to show knowledge processes not only within the company but also knowledge exchange between the company and the outside world.

Table 2.2 Knowledge processes

\begin{tabular}{|c|c|}
\hline Reference & Knowledge processes \\
\hline Wiig, 1995 & $\begin{array}{l}\text { Knowledge creation and sourcing, compilation and } \\
\text { transformation, dissemination, and application and value } \\
\text { realisation }\end{array}$ \\
\hline O’Dell, 1996 & $\begin{array}{l}\text { Identify, collect, adapt, organize, apply, share, and create } \\
\text { knowledge }\end{array}$ \\
\hline $\begin{array}{l}\text { Probst, Raub and } \\
\text { Romhardt, } 2000\end{array}$ & $\begin{array}{l}\text { Knowledge identification, knowledge acquisition, knowledge } \\
\text { development, knowledge sharing/distribution, knowledge } \\
\text { utilisation and knowledge retention }\end{array}$ \\
\hline Tiwar & $\begin{array}{l}\text { Find knowledge, create new knowledge, package and assemble } \\
\text { knowledge, apply knowledge and reuse and revalidate } \\
\text { knowledge }\end{array}$ \\
\hline $\begin{array}{l}\text { Holsapple and Joshi, } \\
2003\end{array}$ & Acquiring, selecting, internalizing, and using knowledge \\
\hline $\begin{array}{l}\text { Kayworth and } \\
\text { Leidner, } 2003\end{array}$ & eation, storage, transfer, and use \\
\hline Newman, 2003 & $\begin{array}{l}\text { knowledge retention, knowledge transfer, } \\
\text { tion }\end{array}$ \\
\hline
\end{tabular}

For the game simulation model, we have chosen those processes which are most important for a product leadership company and which allow players to see the knowledge processes in the company and knowledge exchange between the company and the outside world. The product leadership companies, in order to retain and improve their market position, should respond quickly to the innovations of their competitors, research laboratories or partners - this requires obtaining knowledge from these parties, transferring them to the involved parties and utilizing this knowledge. In order to be able to create new innovative products, product leadership companies have to create and develop knowledge. The new products cannot be made without knowledge about existing products and routines in their development and production. Thus, old knowledge should be preserved in the company. These basic, but important considerations contributed to our choice to model the following knowledge processes: knowledge gaining (as relevant to the knowledge acquisition process in Table 2.2), knowledge development (as a relevant process to knowledge creation in Table 2.2), knowledge utilisation, knowledge transfer, and knowledge retention.

In the model these processes have the following meaning:

- Knowledge gaining. The process of obtaining new knowledge that is relevant for the company from outside (e.g., getting information from professional journals, conferences, exhibitions and so on). 
- Knowledge development. The process of disseminating and developing individual and organisational knowledge inside a company in a particular knowledge domain (e.g., training programs).

- Knowledge utilisation. The process of applying knowledge (e.g., methods to improve performance).

- Knowledge transfer. The process of passing on specific knowledge to other business process areas or making it available for further use in other business process areas. This also includes the transfer of knowledge between knowledge areas in the company (e.g., cross-departmental meetings and shared access to the databases).

- Knowledge retention. The process of preserving knowledge that is relevant for the company (e.g., storing knowledge in databases or information repositories).

Adopting the definition of a process ${ }^{2}$, we can define a knowledge process as a series of actions which are carried out in order to achieve a particular result. Taking into account this premise and two features of the game simulation model, which we specified in Section 2.2.1: 1) the connection between knowledge variables and regular business variables; 2) the connection between players actions-interventions, events and model variables, we can transform Figure 2.1 into Figure 2.2.

Figure 2.2 Place of knowledge processes in the game simulation model

INPUT

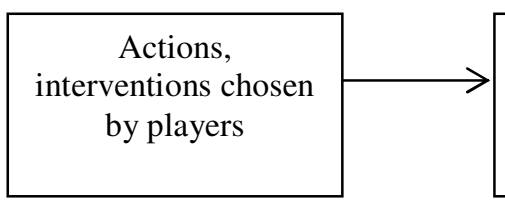

PROCESS

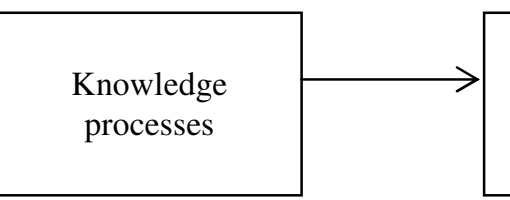

\section{OUTPUT}

on the business variables

Identifying these knowledge processes (see bullets above) for product leadership companies implies knowing which properties or attributes are relevant for describing or characterizing these processes. We need these properties, as they will become the variables that change during the simulation. There are limited references in the literature that define properties of the knowledge processes that can be used for the formal representation of any knowledge process. There are three exceptions: agents or enablers of these processes, knowledge artifacts themselves and transformations, which are "the behaviours that agents perform on artifacts" (e.g., logical reasoning or translation; Newman, 2003, p. 304). Regarding these properties, we do not think that they fully characterise knowledge processes as a process. Moreover, they do not characterise them as a dynamic process, as evidenced by: 1) not referring to the results of the process and the time that is needed for the knowledge process to take place, and 2) focusing on operational steps of a knowledge process which we approach from a functional angle (see also discussion in the beginning of this section 'Building the model').

2 "A process - is a series of actions which are carried out in order to achieve a particular result" (1995). In: J. Sinclair (Ed., at all). Collins Cobuild English Dictionary. London: HarperCollins Publishers, p.1311. 
At the same time we can consider a knowledge process as just another business process. In this case we can represent results of knowledge processes and how they are performed with two widely used performance measures of business processes: effectiveness and efficiency (Turban \& Aronson, 2001, p. 37). They defined effectiveness as "the degree to which goals are achieved" and efficiency as "a measure of the use of inputs (or resources) to achieve outputs". Aversano, Bodhuin, Canfora and Tortorella (2004, p. 3) defined process efficiency "as the ratio between the results the process activities produce - output - and the resources they require - input. The process effectiveness measures the achievement level of the process scope, in terms of users' satisfaction and adequacy to the enterprise's required standards, operative procedures, choices and awaited results with respect to the obtained ones". Another well known definition of these measures was given by Peter Drucker (1998, p. 67). He defined efficiency as "doing things right" and effectiveness as "doing the right things". Based on these definitions we can define the knowledge process property's efficiency and effectiveness - results of the knowledge process and how it was done. Referring to business processes, we can say that their optimisation is realized when goals are achieved faster and at lower costs. This dynamic property is particularly relevant and important for product leadership companies. Therefore, in conjunction with the regular measurements of the process as efficiency and effectiveness we decided to model the dynamic property of a process separately so that it can be influenced by the players/learners as well. To formalise this dynamic property of a knowledge process, we adopted the view that a knowledge process has a velocity characteristic and can be done faster or slower. "Successful companies develop knowledge velocity, which helps them overcome knowledge sluggishness, to apply what they learn to critical processes at a faster rate than their competitors. Underlying this concept is the integration of a company's knowledge processes with its business processes to substantially enhance business process performance" (Tiwana, 2000, p. 36). Rodov and Leliaert (2002) also referred to knowledge velocity, which is the rate at which knowledge is communicated within an organisation. Indeed, depending on the nature of the undertaken actions-interventions by players, a knowledge process can occur in a different time-span, can be achieved by different means and can have a particular outcome.

Taking a knowledge process as a dynamic process, which is carried out to achieve a specific goal, we define knowledge process effectiveness, knowledge process efficiency and knowledge process speed as follows:

- Speed of a knowledge process is determined by the time that is needed for the knowledge process. Speed is a relative notion that, in our view, refers to the ability to do things faster or slower. For product leadership companies the ability to develop a new product and bring it on the market faster is important to maintain a competitive advantage. Development of new products depends on the knowledge processes, thus, the faster knowledge processes run the better.

- Effectiveness of a knowledge process shows the knowledge process results; and

- Efficiency introduces the ratio of the results of the process to the time needed for the specific process. We deliberately include in our definition of efficiency the speed of the knowledge process as the only input and leave out other resources such as money and labour. In our view, taking into account the learning objectives, the game should teach how the dynamics of knowledge processes 
influence business indicators and not how to make a specific knowledge process less costly. Nevertheless, to make the game more realistic, KM interventions or actions that are undertaken by players are associated with labour and costs involved, which are specific for the company that is described in the game. This can be different for other companies, but players cannot manipulate these properties. For example, consider three game interventions: (1) "Hire a high class expert with marketing knowledge on a temporary basis", (2) "Hire a high-class expert with marketing knowledge on a permanent basis", and (3) "Hire marketing personnel on a permanent basis". Interventions 2 and 3 will increase the variables "Number of employees" and "Other expenses" for all periods after their implementation, while intervention 1 will increase these variables for only two game periods. At the same time the highest gain in knowledge development in marketing results from interventions 1 and 2, and the highest gain in knowledge utilisation in marketing from intervention 3. After two game periods in the case that intervention 1 is implemented, knowledge retention and utilisation in marketing will decrease since an expert in marketing leaves the company.

Summarizing, each process can be executed with a different speed; each process may or may not be effective, depending on whether the goal of the process is reached, Finally, each process can be more or less efficient. Therefore, in the game a knowledge process is optimised when its goal is reached faster.

The next step in the model design was to create variables to represent other features of the company, taking into account the knowledge domains and knowledge processes, and to relate them.

Considering knowledge from the knowledge production perspective (Holsapple, 2003) and seeing it as "stocks" and "flows", we have chosen to represent the knowledge of certain domains in the company as a "stock", measured as the level of competence, to which several knowledge processes (that is, "flows") contribute.

To represent propagation of the influence of knowledge flows and stocks, we have to connect knowledge process variables to specific business process variables for each defined knowledge domain (marketing \& sales, research \& development, production), which in their turn should be connected to the variables representing organizational business outcomes. Now the modelling task is to define variables for each knowledge domain to represent formally the business level of each knowledge domain and outcomes of this area of business. In the next stage, variables that describe the outcomes of each business area (i.e. knowledge domain) have to be compared to determine their contribution to and influence on the overall company performance. For example, one of the variables describing the business level of the R\&D domain is the "Company product quality index", which contributes to the variable "Level of sales based on the product quality". This variable is one of the outcome variables of the R\&D domain and is compared to the outcome variables in the marketing and production domains "Level of sales based on marketing" and "Production level". Minimum of these three variables is the variable "Level of sales" which is one of the organisational effectiveness variables. The selection of variables that represent the business and organisational level was based on a literature survey and project work meetings within the KITS project (De Hoog, Shostak, Purbojo, van der Tang, van Heijst, Kruizinga, 2001). The final set of variables, 
which explicitly describe the model and are accessible for the players of the game, are presented in Appendix 1 and can be shown as a four-level model (Figure 2.3). In Figure 2.3 examples of variables within the model are shown as they relate to the knowledge processes (Fig. 2.2).

In this integrated model of the game simulation model we have the following categories of variables:

- Organisational effectiveness variables. These variables represent the relation between the organisation and its environment. They reflect the competitive characteristics of the company and are represented by variables like profit, customer satisfaction index and level of sales.

- Business process variables. These variables reflect the quality of internal processes - the ways things are done and how well they are done inside the company. An example is the production level.

- Knowledge variables. These represent the levels of knowledge in the relevant knowledge domains (for example marketing \& sales) and the quality of applying knowledge in each domain. An example is the level of competence in marketing.

- Knowledge process variables. These reflect the properties of processes involving knowledge in the organisation. An example is the efficiency of knowledge transfer.

In the model, changes in lower level variables lead to certain changes in the higher level variables. Each knowledge management intervention or game event provides different effects on the lowest level variables (knowledge processes related variables). These, from their side, also influence the 'state' of knowledge in the organisation. The state of knowledge in the organisation is represented by knowledge related variables, and determines the quality of the business process: 'how well is the knowledge applied'? Finally, business process variables are connected to the organisational effectiveness variables.

We can illustrate this sequence with the following situation: a key-researcher leaves a company. As a result of this event, which influences the process of knowledge retention and level of competence in the R\&D domain, the company (1) looses knowledge, (2) looses part of the capability to invent new products and (3) loses future market share or profit. To re-gain this knowledge, the company should appoint a new person. This intervention gives an input to a change in knowledge-process variables which characterise processes of gaining knowledge in the organisation. On the next level, new (gained) knowledge changes the knowledge-related variable (level of competence in R\&D). The level of competence in turn influences the business processes. The higher the level of competence in the company, the better the business processes are going. The effectiveness of business processes determine the organisational effectiveness, which is represented by organisational effectiveness variables (for example an increase in profit). 
Figure 2.3 An integrated model of the game simulation model

OUTPUT

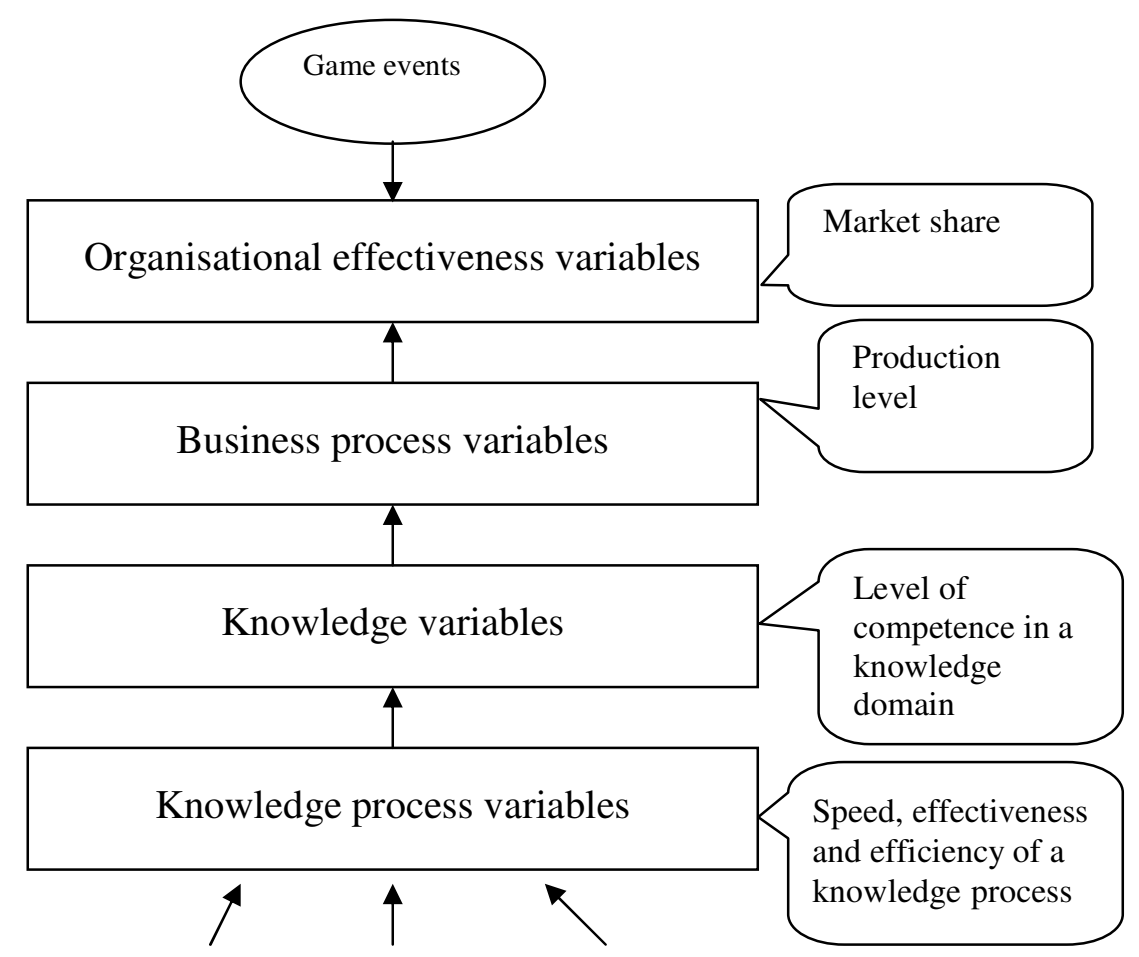

INPUT

Actions-interventions, game events

Some game events can influence business process variables (a leaving expert results not only to changes in the knowledge variables but changes also the value of a variable representing the amount of $R \& D$ personnel) and organisational effectiveness variables (e.g., market share can be influenced by other companies which hire the expert who left the company).

To realise our modelling assumption of knowledge depreciation over time, the knowledge process variables should exhibit decay behaviour. If there is no increase and renewal of knowledge in a company, propagation of values from the knowledge processes (via knowledge and business process variables) to organisational effectiveness variables causes the overall performance of the company deteriorate over time as well (Anjewierden, Shostak, De Hoog, 2002).

The next modelling step is to define the relationships between variables.

\subsubsection{Defining the relations between variables}

In accordance with our modelling assumptions, the link between knowledge processes and organisational effectiveness variables is realised as shown in Figure 2.4, where the level of competence in a specific knowledge domain is influenced by the variables of knowledge gaining, knowledge development and knowledge retention. Speed and effectiveness of knowledge utilisation, efficiency of knowledge transfer from other related domains and level of job satisfaction index influence efficiency of knowledge utilisation. Further, level of competence and efficiency of knowledge utilisation contribute to the 
business process variables. Performance in one domain influences other domains on the business process level. Organisational performance depends on the outcomes of each domain. For example, in a product leadership organisation with the domains Marketing, Production and R\&D, the level of sales is the minimum of the three variables - the production level, the level of sales determined by marketing, and the level of sales determined by $R \& D$.

Knowledge domains are related to each other on the business process level and by knowledge transfer between relevant domains. More precisely, the variables for the R\&D domain, together with relationships between the variables and interventions / events of the simulation, are shown in Appendix 2. Every relation is marked with either a "+" or a "-". This follows a modelling convention known from system dynamics. A "+" means that the two linked variables move in the same direction, if one goes up the other goes also up, if one goes down the other goes also down. A "-" means that they move in opposite directions, if one goes up the other goes down and the other way round. This is an initial specification of the nature of functional relationship between variables.

Figure 2.4 Relationships between knowledge processes and organisational performance

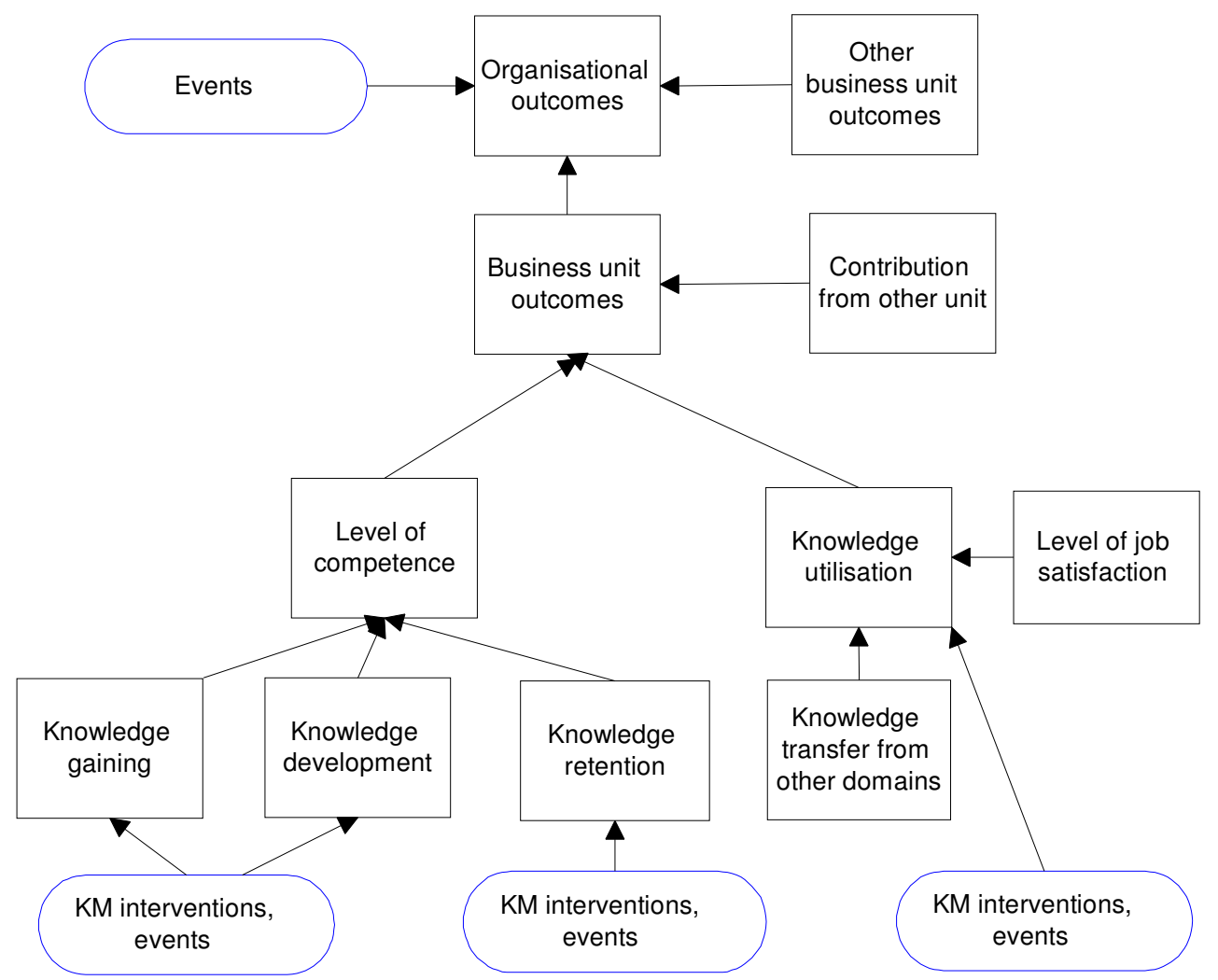

To be able to translate a model into an executable dynamic model, we needed additional specifications. In particular:

- Measurement scales for the variables; 
- Functions describing the 'decay' of variables over time (i.e., what the values of the variables will be when nothing is changed);

- Functional relations between variables that specify the "+" and "-" as well as the combined effect of two or more variables on another variable.

We will deal with each of them in turn.

\section{Measurement scales}

The business process and organisational effectiveness variables should be represented in the model using natural scales and measurements, since our goal is to create a realistic environment. However, the formal representation of knowledge and knowledge processes poses some problems, because there are no known measurement scales and measurements for these concepts. To be able to represent knowledge formally in the model we have to create "artificial" measurements for the knowledge processes and knowledge variables. The variable "Speed of the knowledge process" can, in principle, be measured on the natural time scale - days, months, quarters and so on. In this case we have to create proxy-variables to connect it to the variable "Efficiency of the knowledge process" as they are measured using different units. Another issue to be considered is the comparability of knowledge processes. Since we model them 'in common' without considering each process in detail, the measurements should be also comparable between knowledge processes. For instance, knowledge gaining processes in the company can vary between days and months while knowledge development processes can vary between days and years. What is important for the learning purposes of the game is a relative notion of speed: the faster the knowledge processes are done, the better-an exact (time) specification of each knowledge process is not needed. To make knowledge processes comparable and to ease the modelling process, a decision was made to represent knowledge process variables and knowledge variables on a fictitious scale between 1 and 10, with 10 meaning the best possible value (fastest, most effective and efficient) and 1 the worst possible value (slowest, not effective and not efficient). From the angle of learning purposes this creates a system which helps to observe and understand the phenomenon, without the clutter of too much detail.

\section{Decay functions}

Decay functions describe the behaviour of a variable over time when there are no knowledge management interventions. As the players of the game are not forced to do anything, the model still has to exhibit continuous behaviour. This can be achieved by introducing an autonomous "force" driving change. It should be noted that a decay function can also increase the value of a variable over time, if appropriate. Generally speaking, decay functions fall into several classes:

- Linear decay: the decrease or increase is the same over each time slice. This can be represented by the function $y=a x+b$, where the sign of ' $a$ ' indicates increase $(+)$ or decrease (-) and the value of ' $a$ ', the steepness of the slope, is the speed of the decay.

- Concave decay: the decrease is slow in the first time slices and becomes faster and faster over later time slices.

- Convex decay: the decrease is fast over the first time slices and slows down over later time slices.

Since we could not find any references to functions describing knowledge decay behaviour in the literature, and we could not provide convincing arguments for a 
particular function, we decided to include in the initial model a linear decay function for all knowledge process related variables as well as the variable "Job satisfaction index". We believe that employee job satisfaction also decreases over time if nothing happens in a company. The knowledge process related variables and the variable "Job satisfaction index" receive a positive, negative or neutral influence from input variables. This influence is moderated by interventions chosen by players or events occurring during the game at discrete time periods, when new values of the variables are calculated. Depending on these influences, decay functions can continue their behaviour over time in the case of neutral influences or continue their behaviour over time from a new starting level in the case of negative or positive influences. The magnitude of the influence, which is the value of the input variable, depends on the KM interventions chosen by players in each game period.

Thus in the model the general decay function with KM intervention influences is:

$\mathrm{KRV}=\mathrm{KRV}-\mathrm{C}+\mathrm{KRV} \_\mathrm{A}$,

where

- KRV - knowledge related variable,

- $\mathrm{C}$ - constant decrement, $\mathrm{C}>0$

- KRV_A - Influence from Input variable

The knowledge process related variables have different amounts of decay over time, which is realised by different decrement sizes ("C") in the functions. We believe that decay is relative to what is happening in the environment and how it changes. In our view, the $R \& D$ knowledge domain is more crucial for a product leadership type of company as compared to other domains in the company, since the company competes via its innovative products. At the same time, this domain it is related to changes in the outside world which to a certain extent dictate the company's strategy. For example, consider the market of cell phones or digital cameras. The technical characteristics improve from year to year. If a company does not bring technically updated products to the market, the company will be soon out of business. In our view, in turbulent environments the knowledge on which companies compete in the market changes faster than a company's other knowledge. Therefore, R\&D knowledge for a product leadership company changes faster than its marketing knowledge, due to external influences. Based on this idea, the knowledge process related variables for the $R \& D$ domain have a faster decay (decline) than the knowledge process related variables for marketing and production. The same principle is used to specify the decay of specific knowledge process within a particular domain. For example, the variable "Effectiveness of knowledge gaining" in the marketing domain has a steep decay, while the variable "Effectiveness of knowledge retention" in the marketing domain declines less steeply, because marketing to a greater extent is focusing on the external world and its changes. By such differentiation of decay functions, the relative importance of knowledge process was modelled for each knowledge domain. In other words, steep decay means: 
- The highest priority and greatest importance of a specific knowledge process for the "stock" level - knowledge related variables compared to the importance of other knowledge processes for this domain.

- The highest priority and greatest importance of a knowledge domain for the overall company performance compared to other knowledge domains.

\section{Functional relations}

In order to realise the modelling assumptions, the following functional relations were implemented in the game simulation model:

- The model has Input, State, Constant, Output and Case variables. The value of input variables is modified for each game period based on the game event and/or interventions of players. The input variables change the value of state variables at points in time to moderate output variables' values. Constant variables have a single constant value and are used to calculate values of state and output variables. Case variables are the initial values of output variables specified from the description of the company.

- The lowest-level state variables in the model ('speed' and 'effectiveness' variables) have continuous decay behaviour and are changed by input variables mediated from events and interventions. Decay behaviour means that over time the value of the lowest-level state variables decreases if there are no interventions made by learners or game events. Otherwise the value is the sum of the current value of the variable and the value of the input variables if there are some interventions taken. The latter value depends on the size of the effect of the intervention and event(s).

- The lowest-level state variables - 'speed' and 'effectiveness' variables have a positive relationship with the 'efficiency' state variable and determine its value. The efficiency variable is the sum of the values of the 'effectiveness' and 'speed' variables, which have different weights in the calculation. These weights vary between knowledge domains.

- The 'expense' and 'employees' variables do not exhibit decay behaviour and are calculated directly, based on the initial value of the case variables and input variables, which are associated with the interventions or events.

- The state variable "level of competence" in each domain is computed based on the values of three variables: efficiency of knowledge gaining, efficiency of knowledge development, and effectiveness of knowledge retention in the same domain. For example,

\section{$\mathrm{CM}=\mathrm{K} 1 * \mathrm{EKGM}+\mathrm{K} 2 * \mathrm{EKDM}+\mathrm{K} 3 * \mathrm{EfKRM}$, where}

$\mathrm{CM}$ - average level of competence in marketing

EKGM - efficiency of knowledge gaining in marketing

EKDM - efficiency of knowledge development in marketing

EfKRM - effectiveness of knowledge retention in marketing

$\mathrm{K} 1, \mathrm{~K} 2, \mathrm{~K} 3$ - coefficients

Coefficients for computation of the state variable 'level of competence' differ between knowledge domains to express the idea of different levels of importance of knowledge processes within a specific knowledge domain. 
- The state variables 'level of competence' and 'efficiency of knowledge utilisation' contribute to the calculation of the business processes related variables. The variable 'efficiency of knowledge utilisation' depends on the efficiency of knowledge transfer from other knowledge domains, the level of job satisfaction variable and the speed and effectiveness of knowledge utilisation itself.

These variables have different contributions to the business process related variables, depending on the knowledge domain to realise differences in importance of knowledge domains.

The detailed specification of all functional relationships cannot be presented in this thesis, because it is a confidential part of the KITS project.

\subsubsection{Defining game events and interventions}

In order to implement the game requirements, events and interventions were introduced in the game. Creation and selection of game events and interventions for players was done during the KITS project by several project partners based on the real data available from mass-media, scientific literature and practical experience of the CIBIT consultants/educators (De Hoog, Shostak, Purbojo, Anjewierden, Christoph, Kruizinga, 2002; Leemkuil, Christoph, de Hoog, de Jong, Ootes, Purbojo, Shostak, 2002). From this data, 50 events and 56 interventions were selected to be implemented in the game. Events in the game can be characterised based on the locus of the event - internal or external; the effect of the event - direct, delayed, no effect; and the nature of the event - KM relatedproblem, KM related-opportunity or KM neutral events. All interventions in the game can be divided into several groups based on the aim of the intervention. For example, some interventions aim at the implementation of organisational changes or while others aim at the co-operation of employees and departments within the company.

From the modelling point of view, the game evens and interventions taken by the players do have consequences, because they change the state of the model each game period by generating values of input variables. The 'input' from events and interventions is an influence on the model which can be described as:

- Influence on the state variables 'speed' and 'effectiveness' of a particular knowledge process for one or more knowledge domains, one or more knowledge processes, the variable 'market share' and 'job satisfaction index'.

- Immediate or delayed influence: Events or interventions can have an influence which takes place in the next game period or in later game periods. Moreover, the same event or intervention can have an immediate influence (in the next period) on one variable and a delayed influence on another variable.

- Single or multiple influence(s) (negative or positive), events or interventions, depending on their type, can have a single influence or they can have multiple influences on the same variable over time.

- Magnitude of the influence. The value of the influence can differ.

- Persistence of the influence. The duration of influence can differ. 
All these influences need to be specified for each event and intervention in order to translate them into a formal language and to build an executable game simulation model.

For modelling purposes, the possibility of carrying out interventions in the game is important. For each intervention it is specified how many times in the game the intervention can be taken: once or twice in the game, only within certain time intervals, or every game period. In addition to the interventions, which can be undertaken (selected) by players, events are introduced. The game events are situations that happen unexpectedly. From the modeling perspective, the point of the game in which the event occurs is important. In the game, events can occur if certain interventions were taken, as a follow-up of a previous event, if certain variables reach threshold values, or during any period of the game. Therefore, event enabling and disabling conditions need to be specified for each event. Enabling conditions describe the conditions which have to hold to make an event a candidate to occur in the game. Disabling conditions describe the conditions which have to hold to remove an event as a candidate to occur in the game. During the game, events are selected randomly from a list of enabled events. This is done to create different versions of the game, which makes it possible to play the game several times with the same people without repeating previous experiences. In Figure 2.5 examples of interventions and events are presented with an explanation of their influences and specifications.

Figure 2.5 Examples of interventions and events with their influences and specifications.

Examples of interventions and their specification

Intervention 1 (I22): Conduct an in-house training programme in marketing and sales.

This intervention immediately positively influences the variables "Speed of knowledge development in marketing" and "Effectiveness of knowledge development in marketing" and has, with a delay of one period, a positive influence on the variables "Speed of knowledge utilisation in marketing" and "Effectiveness of knowledge utilisation in marketing"

Intervention 2 (I38): Create databases with information about past and on-going research projects.

This intervention has an immediate positive influence on the variable "Effectiveness of the knowledge retention in $R \& D$ " and a positive influence on the variables "Speed/effectiveness of knowledge development in R\&D", "Speed/effectiveness of knowledge transfer in R\&D" and "Speed/effectiveness of knowledge utilisation in R\&D" delayed for one period.

Example of the specification of interventions is given in Appendix 3. 
Figure 2.5: Continued

Examples of events

Event 1 (E23): After a long period of relatively sparse interaction between production and research, caused by an 'incompatibilité des humeurs' between the department heads, last week's company party renewed informal talk between production and research. Several people reported relief that finally the ice has been broken.

This is an opportunity event which can happen any time in the game and which has immediate positive influences on the variables of speed and effectiveness of knowledge transfer in R\&D and Production.

Event 2 (E17): During recent negotiations with the Unions, it has become clear that they will insist on improving the safety conditions in Coltec's plants as the accident rate has gone up considerably over the last five years.

This event does not have any influence on the variables since it shows that the problem exists and the event is triggered by the values of two variables that exceed the associated threshold values - the level of competence in production and the average job satisfaction index. Besides, this event has a disabling condition - it can not be triggered if earlier in the game participants implemented the intervention "Implement a safety and ergonomic environment" system.

Example of the specifications of events is given in Appendix 4.

\subsection{Summary}

At the end of the modelling process we have created a formal representation of a product leadership company. In this conceptual model, three knowledge domains and five knowledge processes are modelled to represent the knowledge household of that fictitious company. The knowledge process related variables have decay behaviour to show knowledge depreciation over time and are linked to the business process related variables by several equations. The values of the organisational performance variables depend on the values of the knowledge processes related variables. Therefore, if there are no exogenously induced changes in the knowledge processes variables, the overall performance of the company will decrease due to the decay functions. The knowledge management interventions and events have different influences on the knowledge processes related variables and, consequently, influence organizational performance variables.

However, several questions still have to be answered: Does the model represent real-world causality and phenomena? Can we promote the learning of how to solve 
knowledge management problems using this model? How do different conditions and aspects of the game simulation model influence learning? These questions will be answered in later chapters, but first we will explain how this conceptual ("paper") model was transformed into an executable computer simulation model. 


\section{Chapter 3}

\section{Translating the model into a computer program}

This chapter centres on a verification process of the game simulation model. It describes the techniques that were used for the model verification and the software tools which helped to translate the model into a program and debug possible errors. Further it presents the results of the model verification. 


\subsection{Introduction}

Generally speaking, model verification is the process of assessing whether a model operates correctly or as intended (Sargent, 1998; Balci, 1998; Law \& Kelton, 2002; Feinstein and Cannon, 2002; Carson, II, 2002). Model verification ensures that the model is accurately transformed from one form into another. The accuracy of transforming a problem formulation into a model specification or the accuracy of converting a model representation into a micro flowchart and into an executable computer program is evaluated in model verification (Balci, 1997).

Feinstein and Cannon (2002) noted that the process of verification involves debugging the model by isolating and eliminating as many errors as possible. Carson, II (2002, p.52) agreed that verification occurs "when the model developer exercises an apparently correct model for the specific purpose of finding and fixing modeling errors. It refers to the specific processes and techniques that the model developer can use to assure that the model is correct and matches any agreed-upon specifications and assumptions". Thus model verification is a necessary condition for determining validity. There is no validity if the program runs incorrectly. Therefore, verification is designed to ensure that the model is built right. Among many verification and validation $(\mathrm{V} \& \mathrm{~V})$ techniques that are described in the literature on modeling and simulation, Law and Kelton (2002) mention 8 verification techniques that are useful for debugging the computer program of the simulation model. Table 3.1 shows an overview of these techniques and their applicability to game simulation model verification.

Table 3.1 Overview of the verification techniques and their applicability

\begin{tabular}{|l|l|l|}
\hline Technique description & Applicability & If not applicable, why \\
\hline $\begin{array}{l}\text { 1. Write and debug the computer program in modules or } \\
\text { subprograms }\end{array}$ & Applicable & \\
\hline $\begin{array}{l}\text { 2. Structured walk-through of the program by several } \\
\text { persons }\end{array}$ & $\begin{array}{l}\text { Limited } \\
\text { applicability }\end{array}$ & Limited by the project \\
\hline $\begin{array}{l}\text { 3. Runs of simulation under variety of settings of the } \\
\text { input parameters to see that the output is reasonable }\end{array}$ & Applicable & \\
\hline $\begin{array}{l}\text { 4. Trace in the discrete-event simulation: compare outputs } \\
\text { of the simulation after each event occurs with hand } \\
\text { calculations }\end{array}$ & Applicable & \\
\hline $\begin{array}{l}\text { 5. Runs of the model under simplifying assumptions for } \\
\text { which its true characteristics are known }\end{array}$ & Not applicable & No reference system \\
\hline 6. Observe an animation of the simulation output & Applicable & No reference system \\
\hline $\begin{array}{l}\text { 7. Compute the sample mean and sample variance for } \\
\text { simulation runs and compare them with desired or } \\
\text { historical data mean and variance }\end{array}$ & Not applicable & Limited by the project and task \\
\hline $\begin{array}{l}\text { 8. Use a commercial simulation package to reduce the } \\
\text { amount of programming required }\end{array}$ & $\begin{array}{l}\text { Limited } \\
\text { applicability }\end{array}$ & \\
\hline
\end{tabular}

From these techniques two methods are not applicable to game model verification since the model includes 'unmeasured' variables and data from a reference system is not available. The remaining techniques were applied in the model verification process to ensure a correct translation of the model into a computer simulation. The translation of the model into a computer program can be done in three different ways:

- To write the program from scratch in a programming language; 
- To use existing commercial simulation tools or packages;

- To create a tailored simulation building environment.

All three methods have advantages and disadvantages. Writing the program from scratch requires from the modeler knowledge of a programming language and adjustments of the model and the program can often be done only easily and bug free by the person who created the model and wrote the program, due to the often opaque nature of programming source code. The usage of existing application-oriented or generalpurpose simulation packages (Law \& Kelton, 2000) includes additional costs to use the package and time to learn the package's functionalities and, in some cases, additional programming is still required to implement all functionalities needed for the simulation. Creation of a tailored simulation building environment requires additional time to build, but simplifies the work of the modeler and makes it possible to build the models, or adjust the models with the same functionalities by different persons as the environment is often quite close to the "paper" version(s) of the model. In the KITS project the third option was used and a tailored simulation building environment was created. The modeler could not write the program in the programming language, and the usage of existing simulation packages required additional programming to implement specific features of the game events and interventions.

Therefore, within the KITS project several software tools were developed to assist in translating the conceptual model, including variables, relationships between variables and modeling assumptions into a computer program and to tune and debug the model. These tools complemented a simulation package for creating process-oriented KM simulations - KMSim ${ }^{\circledR}$ (Anjewierden, Shostak \& de Hoog, 2002). This simulation package allows a modeler without programming knowledge to build a model and, moreover, to tailor an existing model and create new models supporting the same modeling ideas.

\subsection{Simulation building environment}

A model entry tool, an intervention entry tool, an events entry tool and a simulation tool provided automatic detection of many possible errors which can be made by a modeler while determining the type of a variable, its value range, maximum and minimum values, disabling and enabling conditions for events and interventions.

\subsubsection{Model entry tool}

The model entry tool (Figure 3.1) allows the modeler to create, edit or delete a variable. While creating a variable, the model entry tool provides an automatic selection of:

- The type of a variable - case, state, input, output, or constant (field "Status" in Figure 3.1)

- The category of variables in accordance with the model structure: knowledge process variables, knowledge variables, business process variables, organizational effectiveness variables or case variables (field "Category" in Figure 3.1).

Assigning a variable to one of these categories assumes that we arrange the order of calculations. The case variables are calculated in the beginning of the game to get inferences between knowledge process variables and organizational effectiveness 
variables in order to be able to calculate state variables in the game runs. Input variables and knowledge process variables are calculated first in each cycle of the game, since they contribute to the calculation of all state and output variables. The model entry tool allows one to enter minimum (field "Min" in Figure 3.1) and maximum ("Max") values of a variable (e.g., knowledge process related variables vary between values 1 and 10). One can also enter an initial value (field "Initial" - to present for players of the game the initial situation in the company in numbers), formulae (field "Formula"), precision (field "Precision" - the number of decimal places in the value for knowledge process related variables) and domain (field "Domain", an administrative field for the modeler) of the variable. In addition, the variable can be declared to be visible, that means that players of the game can have access to the variable, or not visible (hides the variable for players). This opportunity can allow the modeler to create 'different' models available for the players without building new models. The players could, for example, have access only to organizational variables, or only knowledge variables.

Figure 3.1 The model entry tool

\begin{tabular}{l}
\hline Model Entry -- KMsim \\
\multicolumn{1}{|l|}{ File View } \\
Variables
\end{tabular}




\subsubsection{Intervention entry tool}

The intervention entry tool (Figure 3.2) and the events entry tool allow the modeler to specify interventions and events and their influence(s) on the model variables: It allows the modeler to select which variables are used and how they are affected. Both tools consist of the same fields and the implementation of influences is realised in the same way. This works because technically they both are "external acts" that change the state of the model, even if conceptually they have different meanings. For each input variable (central pane in Figure 3.2 - Intervention 'Organise apprenticeship system', affected variable 'Speed of knowledge utilization in marketing' KUAsM) affected by an intervention or event, the effect is specified by the previously introduced concepts (section 2.2.3):

- Delay. When an effect occurs (e.g., immediately or after some game periods).

- Initial effect. The initial effect of an intervention is usually positive (e.g., knowledge utilization increases).

- Next effect. Does the effect disappear completely or partially?

- Repeat and reset effect. Effects can repeat many cycles (e.g., paying for subscriptions) and their effect can change over some periods.

- Repeat - number of game cycles, when repeat effect occurs

- Cyclic - effect can repeat cyclically

- Repeat effect - value of the repeat effect

- Reset - 'yes': effect disappears in the next cycle after cycle in which repeat effect occurs; 'no': effect persists for the rest of the game

The tools do differ in the implementation of interventions and events: their occurrence in the game. In the intervention entry tool (Figure 3.2) it is possible to specify each intervention's possibility, frequency and duration. Possibility of interventions can be specified in the field "Possible": an intervention can be always available or unavailable because it has already been implemented (e.g., "Organise apprenticeship system" is done only once). It is also specified whether an intervention can be implemented only a limited number of times (Max), there has to be time between subsequent implementations (Periods) or the intervention is automatically removed after a certain number of cycles (Remove after).

Event occurrence is specified in the events entry tool ${ }^{3}$ by enabling (e.g., "and $(\mathrm{JSI}<6, \mathrm{CP}<5.5)$ ") and disabling (e.g., intervention (I53, active, begin,-1)) conditions. In this example the specifications mean that the event can be triggered only if the threshold values of two variables (JSI and CP) are both below the specified values and it cannot be triggered if the players implemented intervention I53 earlier. In other cases interventions or events specified in the enabling conditions allow an event to occur.

In the example in Figure 3.2, the value of the input variable "Speed of knowledge utilization in marketing" will increase with 0.7 with delay of one game period and will persist after two periods for the entire game with value ' 0.2 ', because the "next effect value -0.5 " decreases the initial effect of 0.7 .

\footnotetext{
${ }^{3}$ Event entry tool is not shown in this work
} 
Figure 3.2 The intervention entry tool

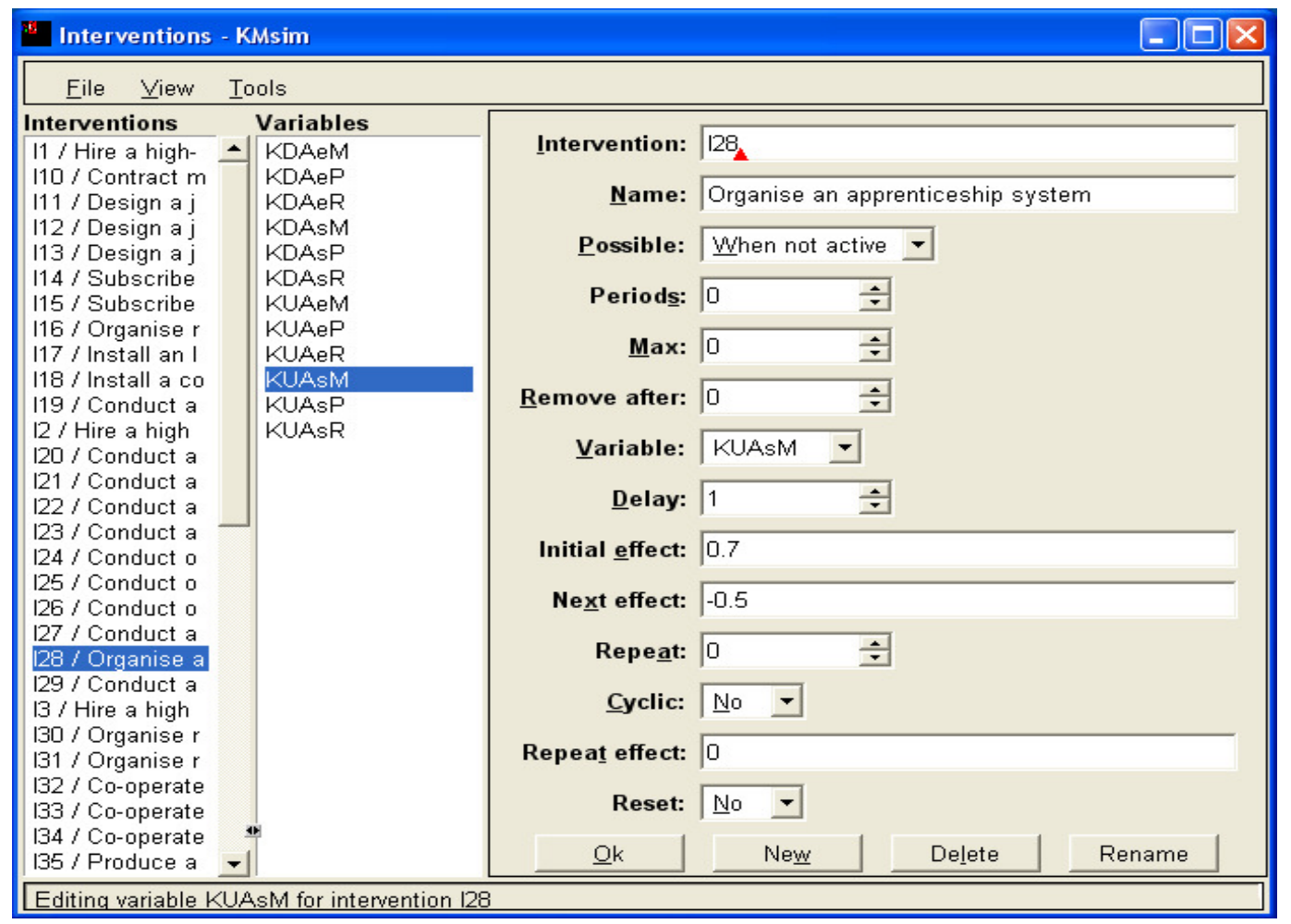

\subsubsection{Simulation tool}

When variables, events and interventions are specified, the modeler can switch to the simulation tool. The simulation tool (Figure 3.3) displays the behavior of the model under several conditions. The modeler can select the model's default (uninterrupted) running by choosing the button "All cycles" without selecting at the same time events and interventions. The modeler can select events (lower left hand pane in Figure 3.3) and interventions (lower central pane) for each model run. It is possible to run one or more cycles (by choosing the button "One cycle") of the model and see immediately the outputs of the simulation in textual or graphical format, which can be chosen in the menu item "View", in the upper pane. The modeler selects the events (in the lower left hand pane; selected events are colored black, events which can occur based on the specification of enabling and disabling conditions are green and events which cannot occur are red) and the interventions (the selected interventions will be represented bold faced in the lower central pane) he or she wants executed in the particular run of the simulation. The modeler also selects variables (bold faced in the browser lower right hand pane) which he or she wants to observe in the upper pane. These facilities are useful for the verification process of the game simulation model in order to trace modeling and specification errors. 
Figure 3.3 The simulation tool

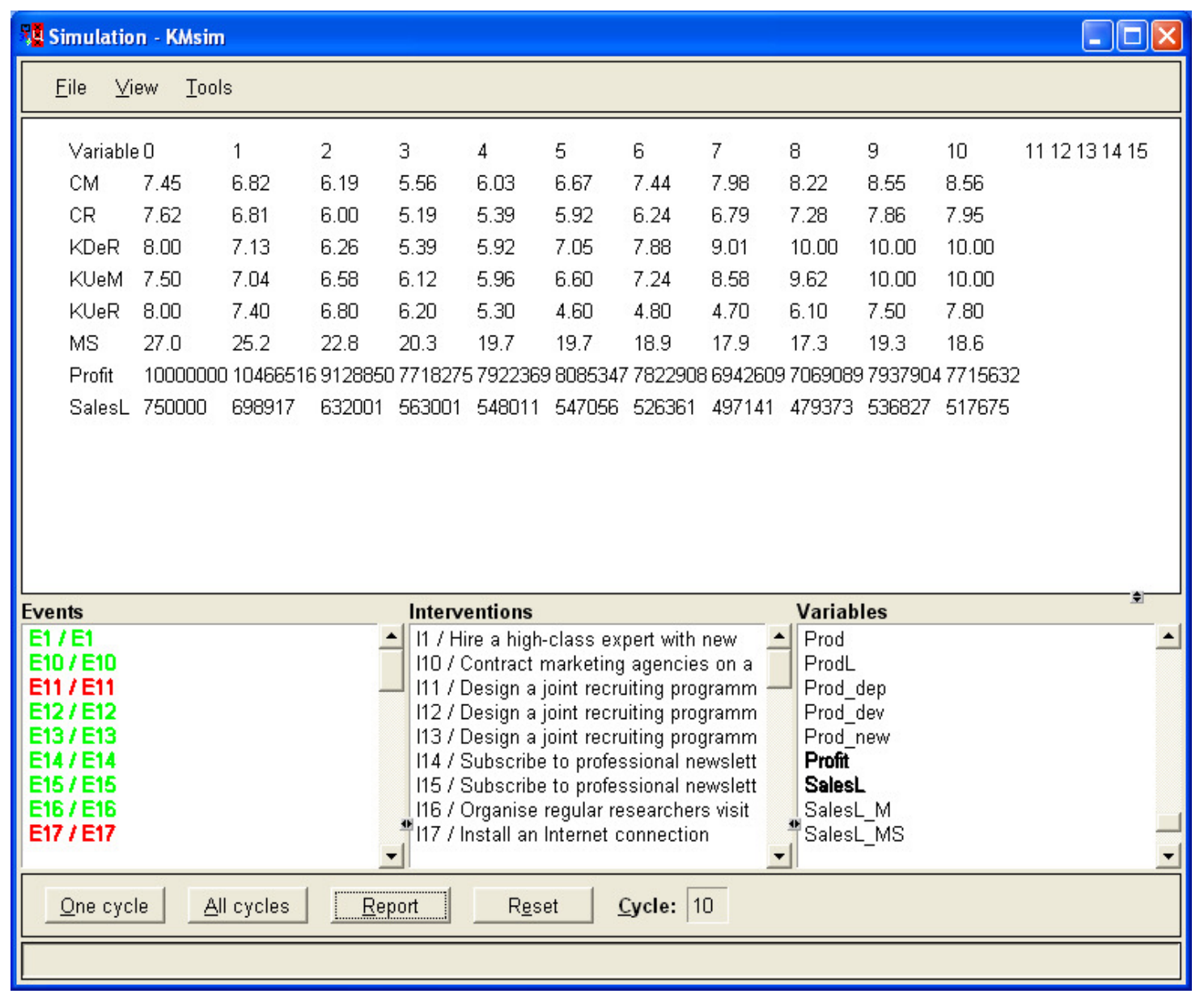

In addition to the textual and graphical representation of simulation outputs, the tool provides visualization of the model inferences, which is realized in two ways: visualization of entire model relationships (Figure 3.4) or visualization of an intervention (Figure 3.5). These visualizations allow the modeler to find mistakes in the model based on the representation of model dependencies. Figure 3.4 represents the semantics of the model: the type of the variables and their relationships. The figure is drawn on the principles of variable dependency. Different colours of the variables are used to indicate the category of the variable. The outer ring contains variables with zero dependency; the next inner ring contains variables which are dependent on the variables in the outer ring and so on. Thus, the modeler can immediately see from the placement of the variables in the picture whether there are errors in the implementation compared with the specification. In Figure 3.4 vertices represent variables and their type (I=input, $\mathrm{S}=$ state, $\mathrm{O}=$ output) and edges represent influences. 
Figure 3.4 The model semantics

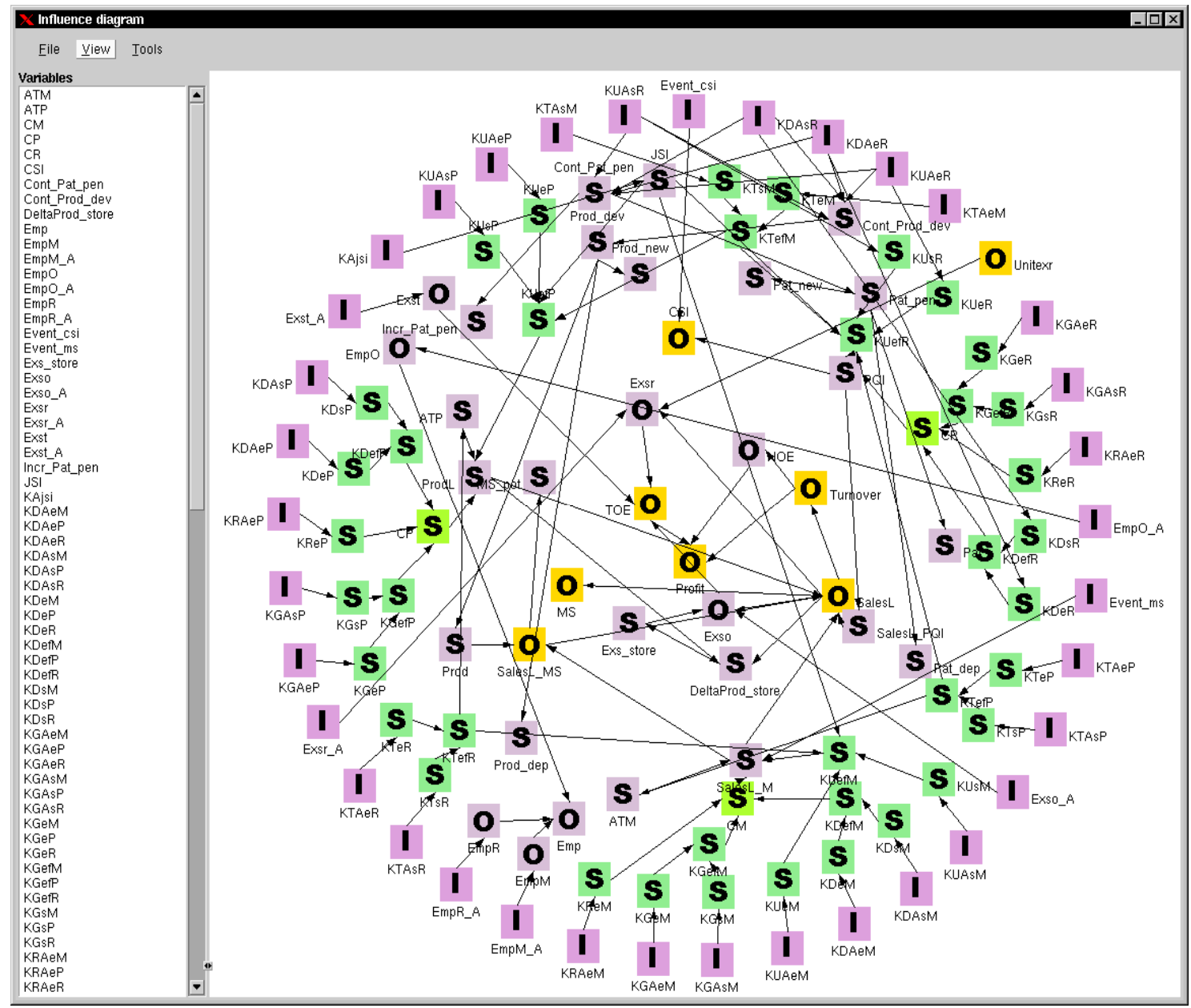

The intervention graphs (see Figure 3.5) are also realized based on the principle of variable dependencies that allows the modeler to recognize possible errors.

In the intervention graph for a specific intervention (highlighted in black in Figure $3.5)$ the vertices represent variables and the edges represent the propagation of influence from the I (input) variables through the $\mathrm{S}$ (state) variables to the $\mathrm{O}$ (output) variables. A triangle in the upper left hand corner of the variable box indicates whether the variable increases (triangle up and green), decreases (triangle down and red) or does not change its value (circle and yellow) as the result of the influence of that intervention. 
Figure 3.5 The intervention graph

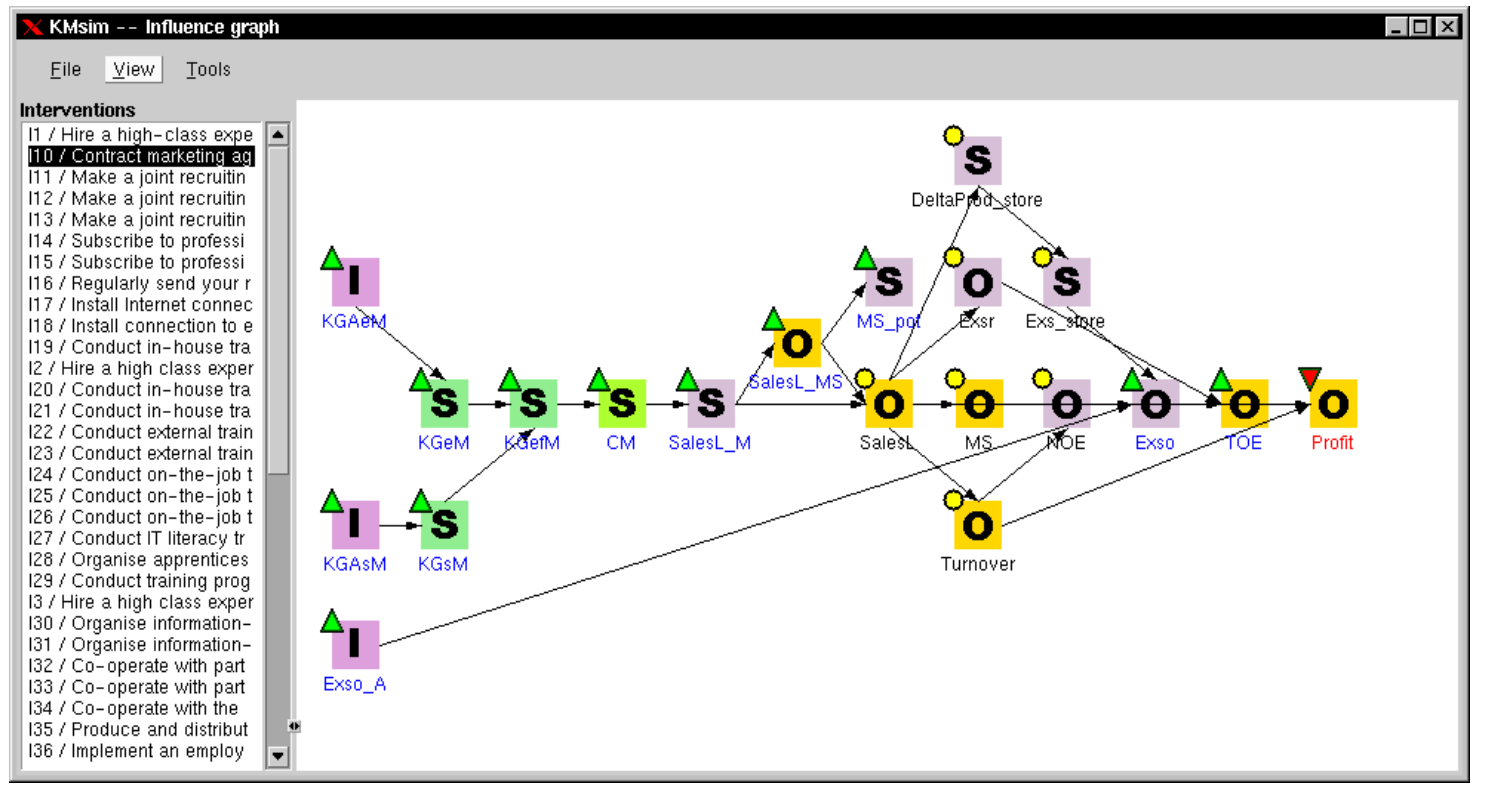

\subsection{Verification of the model}

The simulation building tools and their facilities were used in the verification process of the game simulation model. The model runs in the simulation tool did not show incorrect implementation of the model, but the model behavior in the game environment was still questionable. In addition to this problem, there is always a risk of judgmental biases of the model designers in the design and evaluation processes which could influence the simulation model's validity (Irvin, Levary \& McCoy, 1998). Hence, the game was tried out with potential users (Christoph et al., 2003) in order to overcome 'blindness' of model developers and evaluators from the project team and to assure that the game is operated as intended.

During the first prototype evaluation of the game in the KITS project, the following errors concerning model behavior were detected:

- Disabling and enabling conditions. Disabling and enabling conditions for events and interventions were not working correctly for 30 out of 50 game events; the coupling condition (two events can occur only in the case of a specific event occurrence in the previous game period) was not implemented. Disabling and enabling conditions for interventions were not working properly. In the game all interventions were implemented only once, the repeat implementations of the same intervention led to a decrease of the game budget, but did not have any other effects. Evaluation showed also that some interventions should be excluded from the list of available interventions after their first implementation. 
- Model behaviour. The model didn't behave correctly when teams applied a large number of interventions at the same time. The changes propagated through the system were too large to be realistic.

- The name of the indicator 'profit' should be changed to 'profit/loss', since some teams had a negative profit.

- The behavior of the 'market share' indicator should be corrected, since we did not infer into the modeling assumptions such property of the market as its elasticity.

- The decay function was very steep; teams could not play well against two competing factors at the same time: events and a steep decay function.

Based on these problems, for the second prototype the following features were changed:

- Disabling and enabling conditions were adjusted,

- The behaviour of 'market share' indicator was corrected,

- Input variables received limits (max and min values) to overcome the problem of unrealistic changes when many interventions were applied at the same time;

- The decay function was made less steep

In the second prototype evaluation of the game during the KITS project, attention was given to the plausibility of the model behaviour. We observed 38 games played by students in order to check the model behaviour (reference to D14). Based on results of the model runs and games played, the input specifications for 17 events out of 50 and almost all input data for interventions were partly changed in order to achieve higher model plausibility. In most cases the specified initial influences on input variables were too high and several interventions missed the specification of influences on the knowledge retention.

One of the important questions is how much to test or when to stop testing. The answer to this question depends on the study's objectives. The testing should continue until sufficient confidence in the model's credibility and acceptability is achieved (Balci, 1998).

In the KITS project we achieved satisfactory results concerning the behaviour of the model (Christoph, Leemkuil, Ootes, Shostak, Monceaux, 2003). The extended verification process allowed us to fix errors in the computerized model and adjust the model in such a way that it produced plausible behaviour in our view. As a consequence, it can be said that transforming the "paper" model into a running model was concluded successfully and verification was achieved. However, verification is not sufficient to classify the model as being credible. This is a task for the next step of the simulation development process - validation.

\subsection{Summary}

In this chapter we described the process of the model translation into the computer program and the tools that were developed to translate the model into the computer program and fix/adjust the model. Based on the properties of the tools to prevent errors in translation as much as possible and an initial user test, we can conclude that the KMQuest simulation model was verified.

In the subsequent chapters we will deal with the last step in the simulation development process - validation. 


\section{Chapter 4}

\section{Validation of the game simulation model - design of the study}

This chapter is devoted to the issue of the model's validity. Although the KM Quest game simulation model was validated in the KITS project, there are still many opportunities to investigate the model's validity. We begin with the concepts and methods used in the validation and evaluation of business simulations and the applicability of these methods to the game simulation model. We close this chapter with a description of the validation studies of the KM Quest game simulation model. 


\subsection{The need for game simulation model validity}

In Chapter 3 we addressed model verification, which deals with building the model right. Model validation deals with building the right model (Law and Kelton, 2000, Banks, 1998). Why is validation of the game simulation model important?

Peters, Vissers and Heijne (1998) argued that gaming is based on the idea that students can learn from and practice their skills in a simplified model of reality and later transfer their experiences or knowledge back to reality. Carvahlo (1991) posed that students can use the data provided by the simulation to improve their quantitative analysis and decision-making skills. "They can use their knowledge [...] to make critically reasoned choices of goals, policies, and resource allocations. By reflecting on these choices and subsequent outcomes, they can deepen their understanding of preferences, behaviors, values, ethics" (Carvahlo, 1991, p. 328). In accordance with these ideas, we believe that if we want students to make inferences about reality based on experiences and knowledge acquired in the game, and if we want to be sure that students transfer the findings or knowledge to reality, we have to be sure that the game model is a good, or valid, representation of the real phenomenon and that the model provides acquisition of decision-making skills in the domain of knowledge management.

\subsection{Literature review and practical implications}

Despite the fact that simulation and games have been areas of interest to academicians and practitioners for a long time, there are two aspects that have to be clarified before designing a validation study. The first aspect is derived from the fact that there is no agreement among authors in the simulation modelling field as to the meaning of the term "validity" (Robinson \& Pidd, 1998) and, secondly, several authors admitted a lack of consistent evaluation, i.e. validation theory of simulations (Carvahlo, 1991; Feinstein \& Cannon, 2002). We will elaborate on these aspects in the following sections.

\subsubsection{Validity concepts}

Several concepts of validity are presented in the literature on validation and evaluation research. Briefly, validity can be defined as "the degree of homomorphism between one system and a second system that it purportedly represents" (Stanislaw, 1986).Stanislaw uses the term homomorphism to express that after applying modeling assumptions and the principles of reduction, abstraction and symbolization (Peters, Visser \& Heijne, 1998), the two systems, reality and the modeled version of reality, are not isomorphic. While abstracting and reducing, the designer maps an n-dimensional system (reality) onto an m-dimensional system (model), where $m<n$. If $m$ and $n$ are equal, then the two systems are isomorphic. Stanislaw (1986) considers validity to be a concept that is related to the simulation building process. He distinguishes three types of validity: theory validity, model validity, and program validity. He argues that it is not possible to designate one set of theories or models as 'valid', and another set as 'not valid'. In his view, validity is problem dependent.

Sargent (1991) distinguishes conceptual model validity and operational validity. In his view, conceptual model validity is defined as determining that the theories and 
assumptions underlying the conceptual model are correct and that the model representation of the problem entity is reasonable for the intended purpose of the model. Operational validity is defined as determining that the model's output behavior has sufficient accuracy for its intended purpose over the domain of the model's intended applicability. Pedgen, Shannon and Sadowski (1995) considered conceptual validity to be an adequate representation of real world systems and operational validity to be when data generated by the model adequately represent the real-world system's data. Wolfe and Jackson (1989) reported an experimental study in which they found that algorithmic validity of the model does not influence game performance of players and their perception of game realism. They regarded algorithmic validity as an absence of program errors in the game; in this sense this concept is similar to the concept of program validity by Stanislaw (1986). Babbie (1992) referred to content validity (completeness of the model relative to the modeling purpose) and construct validity (correctness of the model in terms of relations between variables). Initially suggested by Raser (1969), Peters, Vissers and Heijne (1998) discussed game validity from the perspective of: psychological reality ("the game is valid to the degree that it provides an environment that seems realistic to players", p. 23), structural validity (structure is isomorphic to the reference system), process validity (processes in the game are isomorphic to processes in the reference system) and predictive validity (the game produces historical outcomes or predicts the future). Carvahlo (1991) specified that validation of business simulators should be done in order to investigate objective learning validity, which is the ability of the game to provide a valid learning experience corresponding to the learning objectives of the course in which the business simulator is used. Wolfe and Gold (2007) referred to the explanatory validity of models in business games, that is, "the ease with which various phenomena can be explained or rationalized to players" (p. 157). In their view, explanatory validity is connected to a model's complexity in a business game. An increase in complexity affects explanatory validity negatively, since it becomes more difficult to explain the phenomenon and to learn from it. Faria and Wellington (2005) consider internal and external validity of business games and simulations compared to the performance of the participants. Internal validity then relates to the performance of participants in a business simulation and external validity relates to the real-life performance of participants. Feinstein and Cannon (2002), referring to the plethora of terms, proposed to consider validity along two dimensions: game development and game application and their associated internal and external validities (See Figure 4.1).

Feinstein and Cannon (2002) argued that game development is based on principles of representational validity such that internal representational validity addresses the extent to which a simulation shows plausible behaviour and functions in the intended manner, while external representational validity addresses the extent to which the behaviour of the simulation replicates the behaviour of the system that has been modelled. The application dimension requires the system to correspond to the design purpose. In our case the applied system is an instructional system that should be based on principles of educational validity, taking into account learning processes and learning objectives. 
Figure 4.1 Two Patterns of Simulation Game Validation

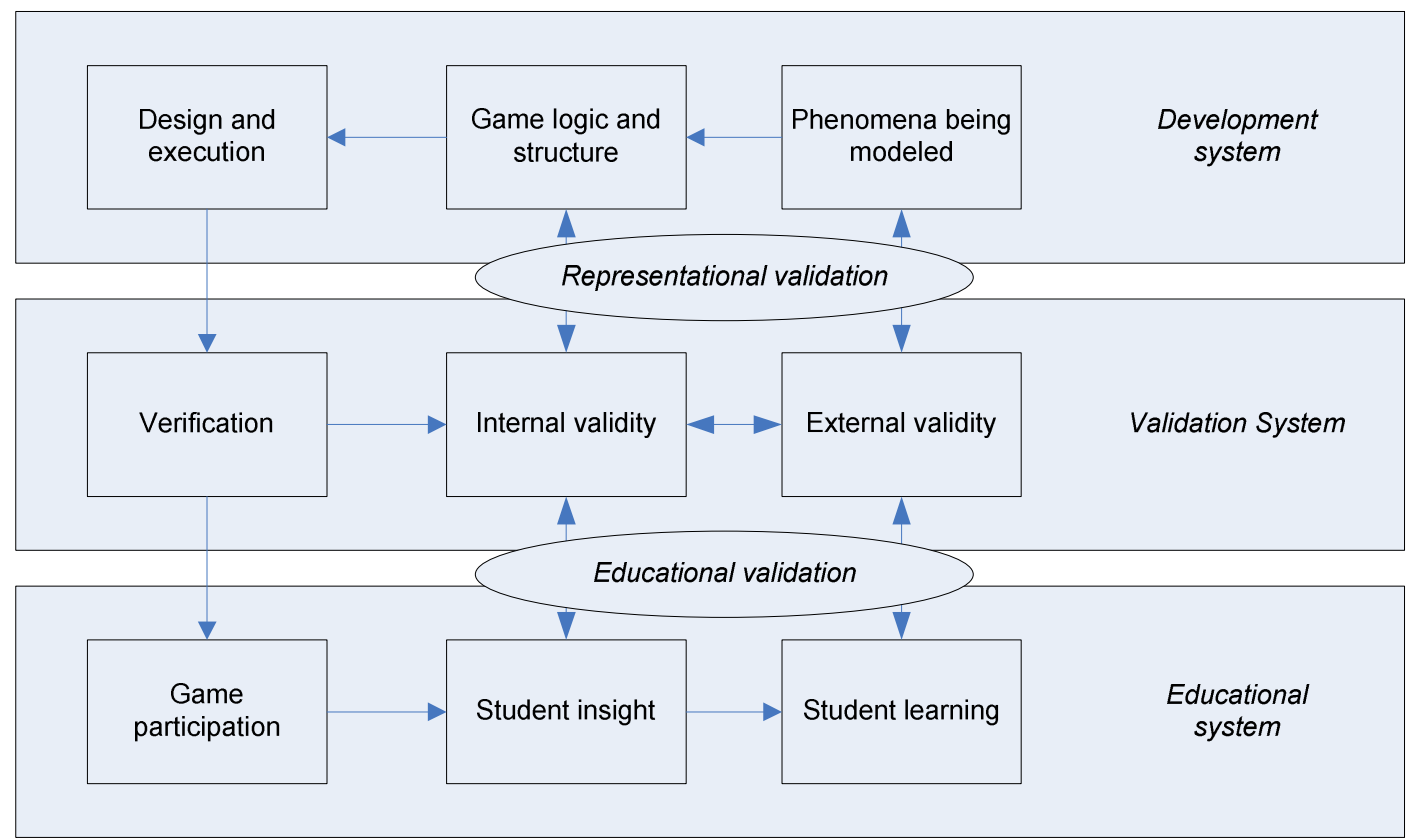

Source: Feinstein, A.H. \& Cannon, H.M. (2002). Constructs of simulation evaluation. Simulation \&Gaming, 33, 4, p.433.

Feinstein and Cannon distinguish internal educational validity and external educational validity. The game has internal educational validity if game participants can discern the phenomena being modelled and develop managerial insight to address them. External educational validation "means either the demonstration that a simulation teaches key business skills (validation as a method of teaching) or that key business skills are needed to perform well in a business simulation game (validation as an assessment instrument)" (Feinstein, Cannon, 2002, p. 437). In Table 4.1 we summarize how, in our view, different concepts of validity proposed by other authors relate to the concepts of validity as proposed by Feinstein and Cannon.

Table 4.1 Concepts of validity

\begin{tabular}{|c|c|c|c|}
\hline \multicolumn{2}{|c|}{$\begin{array}{l}\text { Game development }- \text { representational } \\
\text { validity }\end{array}$} & \multicolumn{2}{|c|}{ Game application - educational validity } \\
\hline Internal validity & External validity & al validity & rnal validity \\
\hline $\begin{array}{llr}\text { Theory, model } & \text { and } \\
\text { program validity } & \text { by } \\
\text { Stanislaw (1986) } & \end{array}$ & $\begin{array}{l}\text { Conceptual and operational } \\
\text { validity (Sargent , 1991; } \\
\text { Pedgen, Shannon and } \\
\text { Sadowski, 1995) }\end{array}$ & $\begin{array}{l}\text { Objective learning } \\
\text { validity } \\
(\text { Carvahlo, 1991) }\end{array}$ & $\begin{array}{l}\text { ive learning validity } \\
\text { hlo, 1991) }\end{array}$ \\
\hline $\begin{array}{l}\text { Algorithmic validity } \\
\text { (Wolfe and Jackson, 1989) }\end{array}$ & $\begin{array}{l}\text { Structural, process and } \\
\text { predictive validity (Raser, } \\
\text { 1969; Peters, Vissers and } \\
\text { Heijne, 1998) }\end{array}$ & $\begin{array}{l}\text { Explanatory validity } \\
\text { (Wolfe and Gold, 2007) }\end{array}$ & $\begin{array}{ll}\text { Psychological } & \text { reality } \\
\text { (Raser, 1969; } & \text { Peters, } \\
\text { Vissers and Heijne, 1998) }\end{array}$ \\
\hline $\begin{array}{l}\text { Content and construct } \\
\text { validity (Babbie, 1992) }\end{array}$ & & $\begin{array}{l}\text { Internal validity } \\
\text { (Faria and Wellingt } \\
\text { 2005) }\end{array}$ & $\begin{array}{l}\text { External validity } \\
\text { (Faria and Wellington, } \\
\text { 2005) }\end{array}$ \\
\hline
\end{tabular}


From this overview of different meanings of validity, it is clear that for this research the concept of validity needs to be properly defined. For our validation study we adopt the definitions of Feinstein and Cannon since they cover all the mentioned concepts, and following their approach we will investigate both representational and educational validity of the game simulation model.

\subsubsection{Validation process}

Assessing the validity of the game simulation model can be done in the framework of two validation approaches. One is derived from the simulation research field and focuses purely on the validation of models as being plausible representations of physical phenomena or, in other words, it focuses on the representational (face) validity of the model. The second approach is derived from the game evaluation research field, where evaluators regard the effectiveness and efficiency of the game and game models as a learning tool-its educational validity.

A combination of both approaches will strengthen our study and will help us to answer whether the model suits the purposes for which it was developed.

\subsubsection{Purpose of the validation process}

In the beginning of this section we commented that there is no united methodology for validating simulations. Game evaluation, among many issues, concerns the validity of the game, and some authors use terms such as evaluation and validation interchangeably or synonymously (Feinstein, Cannon, 2002; Größler, 2001).

Carvahlo summarizes (1991, p. 329) that "there is no generally accepted theory or methodology for validating computerized business simulations". In our view, this problem is due to inconsistent terminology in the field and secondly is partially derived from a statement, upon which practitioners and researchers in the field of gaming and simulation agree, that simulation models, simulations and games, should be developed and later validated or evaluated for specific purposes. These purposes are different from simulation to simulation and from study to study. A model that is valid for one purpose may not be valid for another (Law \& Kelton, 2000). Feinstein and Cannon (2002, p. 437) underlined that "a game might receive a very positive evaluation as a learning tool, but it might fare quite poorly as a tool for modelling actual real-world phenomena". Klabbers (2006, p. 149) distinguished the design and the analytical science tradition in game and simulation research. He argued that "both domains pursue different research objectives and different criteria for evaluating success of their efforts. The analytical sciences develop, test, and justify theories. The design sciences build and evaluate artifacts for well-defined context of use and intended audiences. Usability is a key of their success". Sargent (1991, p. 37) argued that if a model is developed for a specific purpose or application, then its validity should be determined with respect to that purpose: "If the purpose of a model is to answer a variety of questions, the validity of the model needs to be determined with respect to each question". Wolfe and Jackson (1989) mentioned that validity has to be judged in a comparative or task-related sense - the validity of a simulator is a function of what designers want the model to accomplish. Referring to Cronbach (1990), who determined that a learning tool is valid if it is suitable and relevant 
for its predetermined purpose, we assume that a game as a learning tool is valid if it possesses educational validity.

During the KITS project (Christoph et al., 2003) the game simulation model was validated in two prototype evaluations with project partners, managers of several companies and students of two universities. In these evaluations, validation focused on representational validity and program validity of the model, and not particularly on the educational validity of the model. After the second prototype evaluation, the model was considered to be educationally valid as the game produced some learning. In this research we will primarily focus on the educational validity of the model and opportunities to increase it, because the KM Quest game is an educational tool. At the same time, since we believe that educational validity could depend on representational validity, we will investigate the representational validity of the model as well as its possible influence on the educational validity.

\subsubsection{Validity assessments}

\section{Representational validity}

The simulation research field provides us with a wide range of methods to assess the representational validity of models. Assessment of representational validity is usually performed with so-called validation and verification methods and techniques. Roughly speaking, they fall into two categories: techniques that help us to answer the question of whether we build the model right and those which help us to answer whether we build the right model. The most extensive overview of validation and verification methods in the simulation research was given by Balci (1998). He divided these techniques into four categories: informal, static, dynamic, and formal. Concerning these techniques, we conclude that many of them are not applicable for the present study since they are based on the use of available data from existing systems. The model that is addressed here is not a representation of an existing system, because we built a model for a class of organisations rather than for an existing company. In the gaming research field three studies related to the validity of game models are particularly relevant (Thavikulwat, 2002; Sandole, 2003; Faria \& Wellington, 2005). Thavikulwat (2002) stated that "the task in validating a model for gaming simulation is to show that the results of the model are not aberrant when compared to what is known about the subject" (p. 16). In his study, while assessing validity of the model, he compared outcomes of a currency exchange rates model used in the game to the monetary theory. Sandole (2003), in his validation study, compared the findings of prisoners' dilemma simulations with findings from other studies in terms of the direction and magnitude of relationship and the ranking of dependent and independent variables. Faria and Wellington (2005) compared data from simulation game outcomes of two business games with data from real companies' gathered within the Profit Impact of Marketing Strategies project of the Strategic Planning Institute and findings of this project. Common to these studies is the assessment of the validity of the game models by comparing game findings to the outcomes and findings of existing theories or real data. In the case of the KM Quest game simulation model, we are dealing with building a new theory and we are limited by the abstract character of our modelled phenomenon, and the lack of both similar models in companies 
and data measuring knowledge processes. This makes a comparison of the simulation outcomes to real system outcomes very difficult and time consuming since we first have to create such models for several companies and monitor the measurements for several years. Therefore, the attention in the validation study should be given to informal techniques, which can be applied to test representational validity.

Peters, Vissers and Heijne (1998), referred to two possibilities regarding game validity: peer debriefing and member check. Peer debriefing occurs when the validity of the game is discussed with other game builders or experts on the subject or the game and they judge the game from their expert view. The member check occurs when the game concept is presented to future game players and their opinion about the game validity is sought. Some authors (Feinstein \& Cannon, 2001; Francis \& Couture, 2003) argue that judgments on validity of models should be made by domain-experts, while the players or users of simulation and games assess perception of validity, which can be expressed by notions of verisimilitude, plausibility, possibility and believability.

During the KITS project the representational validity of the model was tested using informal techniques with project partners. In this research we will investigate representational validity of the game simulation model further by using informal validation techniques with experts in the KM domain and perceived representational validity with game players.

\section{Educational validity}

There is no well-accepted technique to test the educational validity of games and in particular, game models. Authors in the area of educational research utilize formative and summative evaluation of games and their effectiveness and efficiency as learning tools, which is similar to the concepts of educational validity. Größler (2001) combined these concepts and emphasized that formative evaluation should take place during the development of business simulators in order to assure internal validity and an appropriate degree of fidelity, while summative evaluation should happen during the stage of deploying a business simulator. In this case the focus lies on the learning process and on external validity. In his view, a simulator is internally valid if it shows sound and comprehensible behavior, what we refer to as internal representational validity and fidelity "which indicates an objective similarity between simulator and reality" (Größler, 2001 , p. 4) is what we refer to as external representational validity. He defined a simulator to be externally valid if the insights generated from its application can be transferred to other systems, especially those in reality. This is what we label external educational validity.

Another approach which combines insights from the educational and gaming fields is theory-oriented evaluation for the design of and research in gaming and simulation proposed by Kriz and Hense (2006). Their approach is strongly related to the design process of a game and the situation in which a game is used. They proposed quality criteria meant to guide and support the design process of a game. Only two out of these 50 criteria, reflect to some extent a game model that is designed (p. 278): "The simulation offers an adequate link to reality for the target group; rules, roles, and simulated resources correspond to real, authentic situations"; and "Main processes and interconnected factors of reality are translated into the game model correctly". These two criteria reflect the 
representational validity of the model. The remaining criteria guide a designer to develop a game which is educationally valid and are statements upon which a game should be checked during the design process.

In assessing educational validity of simulations and games, educators mostly rely on acceptance and self-assessment questionnaires and game performance scores (Wolfe \& Jackson, 1989; Romme, 2004; Couture, 2004; Faria \& Wellington, 2005).

Faria and Wellington (2005), in a discussion about past research in the field of game validation, described two approaches that have been used to examine the external validity of business games and two approaches that have been used to examine the internal validity of business games. According to these approaches the simulation game is externally valid if a successful real-world business executive is also successful when participating in the simulation game, or in a longitudinal research design the game is externally valid if a student's business game performance promotes his of her subsequent business career success. As for the internal validity of the game, a game is valid if better students outperform poorer students or a game is internally valid if participants' decisions in the game adapt to the changes in the simulation environment over time (Faria \& Wellington, 2005).

Referring to these approaches assessing internal and external educational validity of games, all of them are not perfect or unbiased. From one perspective, as argued by Größler (2001), the acquisition of knowledge cannot be measured using only performance scores because performance scores do not assess changes in the cognitive structure of a learner, and, from another point, "not every change in performance is related to learning", and "not every change in performance affects knowledge and, indirectly behavior" (Größler, 2001, p.6). Not to mention that in the given approaches, the representational validity of games is taken for granted and is not the subject of investigation.

Carvahlo (1989) noted that business simulators are created to foster the development of managerial competencies, which can be divided into two types: those which can be objectively measured and those which are assessed by judgment. Objectively measurable competencies include all quantitative analysis and analytic decision-making skills. To measure these skills educators developed different tests, called knowledge tests. These knowledge tests evaluate the ability of students to recognize a problem and their ability to make a right decision. The advantage of these tests is their ability to assess the qualitative goal of knowledge accumulation.

Thus, in our study we will use measurements that allow us not only to investigate performance in the game, but also assess the ability of students to make a right decision. However, it is very difficult to speak purely about the educational validity of a model, because the model is embodied in the game and learning environment. The model can not be completely separated from the game environment to find out whether the model possesses educational validity or not. Researchers in the gaming field attend to factors which can affect the educational validity of game or computer simulations.

Maier and Größler (2000) defined in their taxonomy of computer simulations that the characteristics affecting learning are located in three aspects of business simulations: the underlying model, functionality, and human-computer interaction. As Größler (2001) pointed out, systematic variation of exactly one characteristic of a business simulator makes it possible to find effective characteristics by which a business simulator can be 
improved and to identify features of a business simulator which makes it effective in a particular learning situation. This is also true for the KM Quest game. Thus, in this research we will investigate two factors which could affect educational validity of the model. One is an internal model factor and belongs to the "underlying model" aspect as defined by Maier and Größler (2000). The second factor is "external" from the model point of view and belongs to the instructional category, which is not mentioned by these authors, but is important for systems which are designed to teach about specific context or skill training, as the KM Quest game is. We deal with these factors in turn.

\section{Model's fidelity}

In section 2.3 we referred to the notion of simulation fidelity, which has to be taken into account during model building, because there are no doubts about the relationship between simulation fidelity and learning. Training of different knowledge and skills requires different levels of fidelity in simulations (Hays and Singer, 1989). Consider flight simulators and business simulators. "While flight simulators try to be as realistic as possible - including almost every detail-computer simulations of business and economic systems try to abstract from details. This abstraction allows a focus on important structures and behavior modes" (Maier \& Größler, 2000, p. 136). Several researchers reported that "a high level of fidelity does not automatically translate into more effective training or enhanced learning" (Feinstein and Cannon, 2002, p. 426). Alessi (1988) pointed out that for a novice student, while low fidelity instruction produces learning, some increase in fidelity might yield better learning. High fidelity experience can lead to confusion and stress which rules out learning. A novice trainee can be over stimulated by high fidelity that hinders effective learning and training (Martin \& Waag, 1978). Alessi and Trollip (1991) pointed out that there is dependency between the level of fidelity and transfer of learning. They call it "a dilemma in simulation design. Increasing fidelity, which theoretically should increase transfer, may inhibit initial learning which in turn would inhibit transfer. On the other hand, decreasing fidelity may increase initial learning, but what is learned may not transfer to the application situation if too dissimilar" (Alessi \& Trollip, 1991, p. 137). In the case of the KM Quest game simulation model this dilemma is even more challenging since knowledge process variables do not exist in reality.

In our discussion in section 2.3 we distinguish physical and functional fidelity (Hays \& Singer, 1989). In our view, by quantifying and specifying knowledge and knowledge processes we decrease the physical fidelity of the game simulation model, but increase the functional fidelity of the model. Since fidelity is also defined as a degree of realism that a simulation presents to the learner (Feinstein \& Cannon, 2002), by introducing knowledge and knowledge process related variables we decrease the level of realism in the game. The game simulation model consists of knowledge process related variables, like 'speed of knowledge gaining' and 'efficiency of knowledge utilisation', which have not been used before to describe knowledge processes. Does this affect the representational and educational validity of the game simulation model? Do these variables confuse learners and hinder learning or do they contribute to better conceptual and strategic knowledge in the KM domain? 
We assume that there is a relation between availability in the model of the knowledge process related variables (unfamiliar variables) and the educational validity of the model. We can investigate this kind of relation in the experimental study. Do these variables hinder internal and external educational validity of the model, do they contribute to the educational validity of the model or is there a different kind of relation between these concepts? Regarding the availability of the knowledge process related variables in the model, while the model exhibits the same behaviour for all players of the game, the model's representation for the players is different. Thus, the actual four layer model (see Fig. 2.3, p. 23) is working in all games, but some groups of variables are not visible and not accessible for the players. By visualising or making variables inaccessible we vary the level of realism the simulation presents to the learner, the model's fidelity and therefore the mental models and conceptual knowledge that players can build while playing the game. Based on the idea that functional fidelity is more important for the training of decision-making skills than physical fidelity, our assumption for the experimental study is that by introducing these variables to players we increase the internal and external educational validity of the game simulation model and decrease the representational validity of the model.

\section{Mode of playing}

The next options we can investigate in order to find out what influences educational validity of the game and of the game simulation model are to test different functionalities of the game or to test different features of the human-computer interface. Different functionalities of the game and the whole learning environment were investigated in the study described by Leemkuil (2006) and different features of the human-computer interface were reported in studies conducted by Purbojo (2005). In this study we will investigate an instructional aspect - the mode of playing. Although Maier and Größler (2000) in their taxonomy covered some instructional aspects in the functionality category they did not consider different modes of playing. They did consider, under the functionality category, single or multiple person participation, but not cooperative or collaborative aspects of games. Kriz and Hense (2006) stated in their quality criteria that simulation should offer adequate adaptability for changed framework conditions and should offer a variety of interactions between participants. In addition, some authors reported that team size affects learning (Wolfe, 1982). Hence, we will investigate how different modes of playing affect learning or the educational validity of the model.

In the KITS project it was defined (Haldane, 2000) that the game should include a collaborative aspect and team playing, but also allows an individual mode of playing. As argued by van der Hulst, de Hoog and Wielemaker (1999), a high level of fidelity is needed for the same understanding of the task if a task includes team performance. In this sense properties of the model and the leaning context in which the game is played are related. Additionally, nothing is known about differences in perception of games by teams and individuals. Does playing in teams affect perception of the game? How do different learning contexts relate to the educational validity of the game? Do those modes of playing provide equal support for educational validity or does one of them provide better educational validity? Our assumption for the experimental study is that teams dealing with a high level of functional fidelity will perform better than individuals with 
the same level of functional fidelity, and teams will assess the representational validity of the game simulation differently from individuals, because teammates exchange their ideas about the model and variables.

Hence, in our experimental studies we will investigate how different levels of physical and functional fidelity of the model and different modes of playing are related to the educational and perceived representational validity of the model.

\subsection{Concepts of the KM Quest game simulation model validity}

In the KM Quest environment, learning to solve problems is done on two levels: the strategic level and the conceptual level. While playing the game, players should follow the normative knowledge management model, which is a systematic approach to problem solving in the KM domain and is realised as a sequence of steps in a problem solving process. Acquisition of decision-making skills in the KM Quest environment occurs when players relate strategic and conceptual knowledge which are supported and provided by the game simulation model. In this sense, the game simulation model supports the knowledge management model, because strategic knowledge is useful only when a task performer can reason about a specific domain (i.e. possesses conceptual knowledge). At the same time, during one of the steps of a decision-making process conceptual knowledge is supported by the supportive knowledge management model which guides the players through indicators of the game simulation model and helps them with the analysis of KM "bottlenecks" and the selection of the indicators.

Thus, players in the game following the normative KM model should develop strategic knowledge in the KM domain, which we define as the ability to select and apply appropriate KM interventions in order to solve KM problems. The acquisition of strategic knowledge is only possible when players possess conceptual knowledge in the KM domain. Conceptual knowledge consists of knowing KM bottlenecks and KM interventions, the relation between them and the propagation of the effects of $\mathrm{KM}$ interventions on company performance. The acquisition of conceptual knowledge occurs when players work with the game simulation model, which presents to the players indicators describing the 'state' of knowledge in the company, its business and organizational outcomes. The values of indicators change over time depending on the behaviour of players in the game: whether they applied KM interventions, whether they applied 'right' interventions or whether they choose to do nothing. When players connect the past and current game period situation (values of indicators) and reflect on the game situation and their behaviour, they acquire conceptual knowledge (see Figure 4.2). 
Figure 4.2 Learning to solve problems in KM Quest

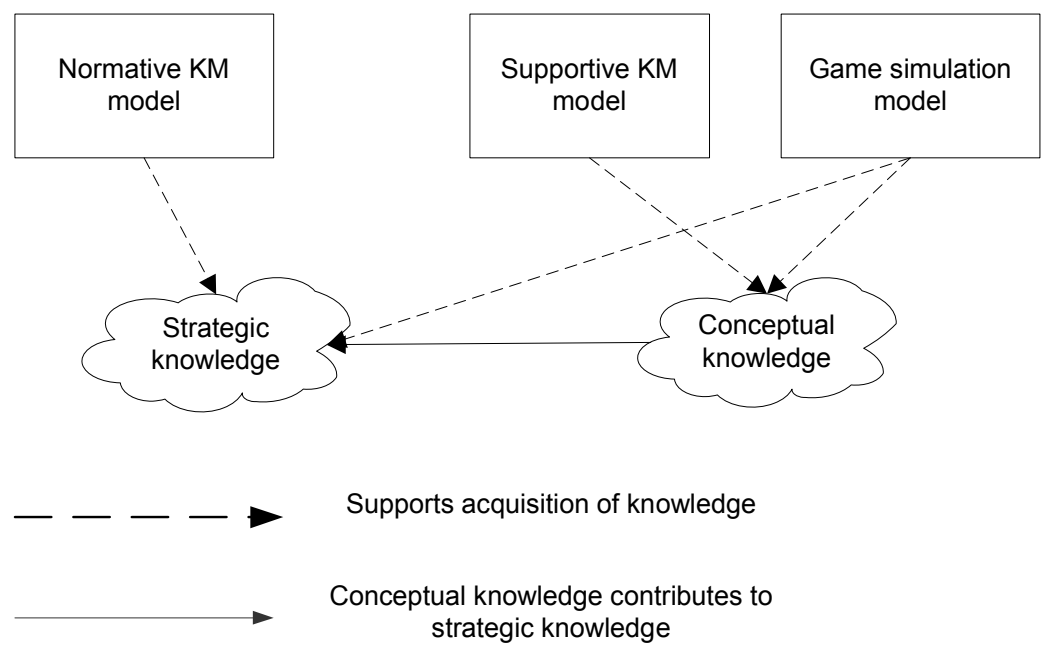

Based on ideas that players of the game need to have a representatively valid model to learn (Peters, Vissers \& Heijne, 1998), and that external representational validity "is a key issue in establishing internal educational validity" (Feinstein and Cannon, 2002, p. 431), we can hypothesise that educational validity cannot be met if the model does not possess to some extent representational validity. We hypothesise that students will be able to understand the modelled phenomenon and its inferences (the model has internal educational validity) and they will develop decision-making skills in the knowledge management domain (the model has external educational validity) if the model provides a valid representation of real world phenomenon (the model has internal and external representational validity).

In our research, we define representational validity as a valid representation of a modelled phenomenon, consisting of:

- Internal representational validity - the model provides a plausible representation of relationships between knowledge, knowledge management interventions, internal or external events and organisational performance;

- External representational validity - the model resembles a realistic organisational situation.

We judge the model as being educationally valid if players of the game will be able to understand relationships between $\mathrm{KM}$ problems, KM actions and interventions and their influence on KM processes and overall company performance, play the game successfully and develop knowledge management problem-solving skills:

- Internal educational validity - the model supports the acquisition of conceptual knowledge (e.g. players discern relationships of the modelled phenomena and address them in the intended manner);

- External educational validity - the model supports the acquisition of strategic knowledge (e.g. players develop decision-making skills in the knowledge management domain). 
Hence, in the following experimental studies we will investigate (1) the educational validity of the model with respect to the notions of conceptual and strategic knowledge which reflect internal and external educational validity of the model, (2) representational validity of the model, and (3) what kind of relations between representational and educational validity of the model exist.

\subsection{Research framework and research questions}

Above, we have discussed the purpose of the validation study, the concepts and methods of validity assessments and the applicability of these concepts and methods in the current research. We defined representational validity and educational validity of the model and hypothesized that educational validity could depend to some extent on the representational validity. However, this hypothesis has to be tested. For this we designed a validation study, which is summarized in Figure 4.3. We will assess the representational validity of the models with experts in the KM domain by using informal methods to find out to what extent the model possesses representational validity. To find out what kind of relationships exists between representational validity and educational validity we will assess perceived representational validity using informal methods with players of the game and compare these assessments to the assessments of the educational validity. By doing this we can understand the nature of the relationships between representational validity and educational validity, if they exist. In addition, as educational validity of the present model cannot be objectively measured separately from the game environment, we will investigate how different factors could affect the educational validity of the model and examine how these factors could affect the perceived representational validity of the model.

Hence, the research questions in this study are:

- To what extent does the model possess internal and external representational validity?

- Which model, in terms of possessing different levels of fidelity, provides higher internal and external educational validity and higher internal and external representational validity?

- Which mode of playing provides higher internal and external educational validity and higher internal and external representational validity?

- What kinds of relationships exist between the representational and educational validity of the model?

We will answer the research questions and investigate the relationships between educational and representational validity in several studies described in the following chapters. 
Figure 4.3 Research framework
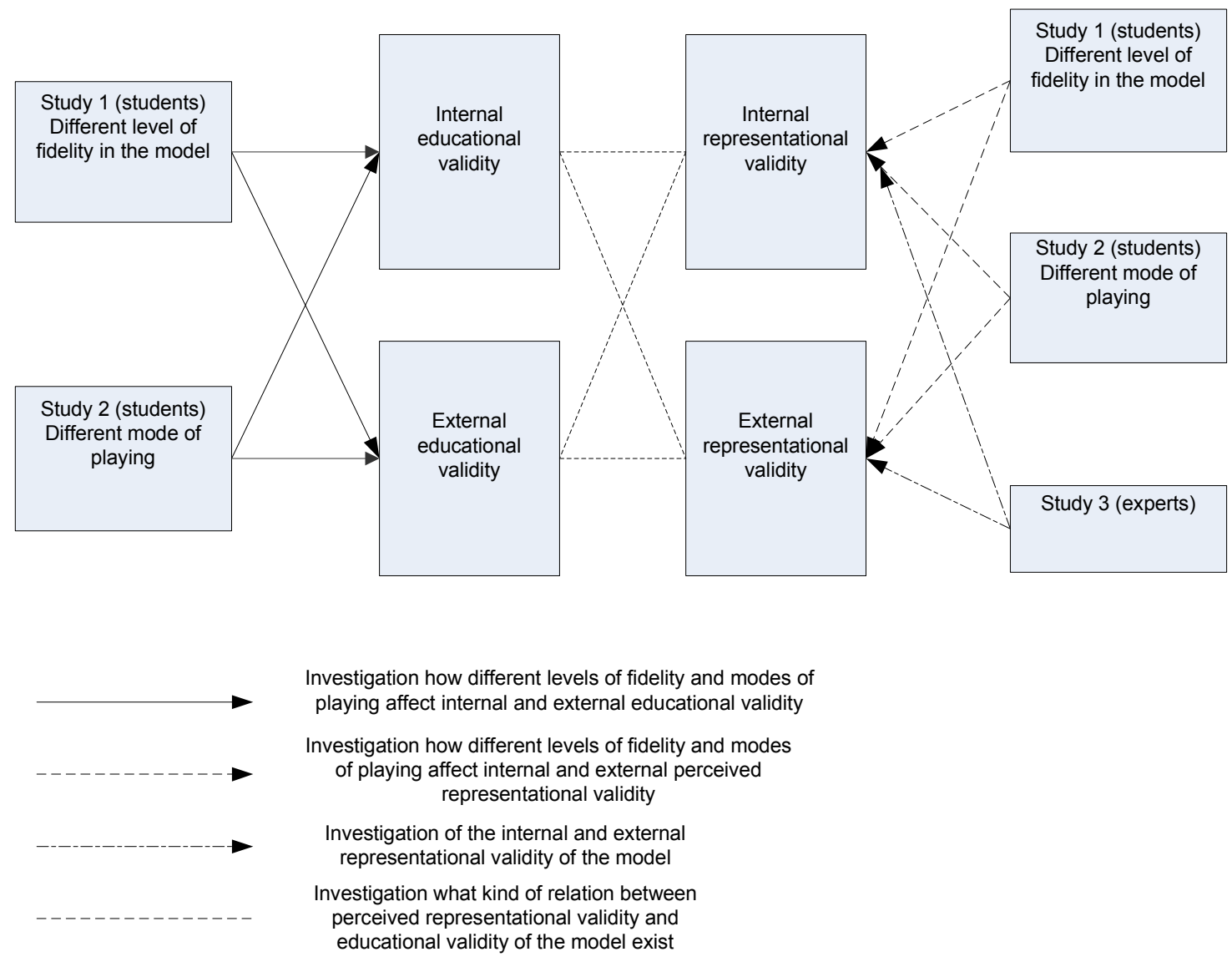

\subsection{Summary}

The validation study of the KM Quest game simulation model aims at investigating its educational and representational validity in different conditions. This provides us with opportunities to find out how we can optimize the educational validity of the model in cases when the representational validity of the model is affected by different conditions thus influencing educational validity.

In the first experimental study we will investigate how an internal factor, from the model point of view- the model's fidelity - influences the model's educational validity and the model's perceived representational validity. Furthermore, we will investigate relationships between educational and representational validity. This study is described in Chapter 5.

In Chapter 6 we describe the second experimental study in which we address how an external factor from the model point of view - the mode of playing - affects the model's educational and perceived representational validity.

In Chapter 7 we will present an exploratory study in which experts in the KM domain assess the model's representational validity. 


\section{Chapter 5}

\section{The model's fidelity: investigating validity}

This chapter reports on the first experimental study that was carried out in the validation process of the KM Quest game simulation model. The aim of the study was to investigate how the presence of the knowledge process variables influences the perceived representational and educational validity of the model. The presence of these variables in the model is related to the model's fidelity and complexity, and these variables are expected to influence both validity concepts. The chapter begins by describing the purpose of the study and the expectations for the outcomes. Next, the experimental design and context of the study are described. Finally, the results and findings of the study are presented and discussed. 


\subsection{Introduction}

\subsubsection{Purpose of the study}

A modeler who creates a model for educational purposes has to be sure that the model suits its goals: it provides understanding of the modeled phenomenon and supports knowledge and skills transfer. However, without trying out the model on the end-users, in our case the players of the game, he or she cannot find out if the model is successful or not. For the KM Quest game simulation model this task is even more challenging since we incorporated into the model knowledge process related variables, which are not easily observable and have never been used in the real-world until now. Does this influence the validity of the model? Do learners/players benefit from these variables in terms of learning how knowledge and knowledge processes are related to business outcomes of a company, or did we create more obstacles to the educational validity of the model? Did we bring an additional complexity into the model, do these variables confuse and distract players' understanding of the phenomenon or do they provide better learning? Do knowledge process related variables affect the representational validity of the model players' perception of realism of the game and how this influences learning? Can we optimize representational and educational validity of the model of the game in such a way that unfamiliar variables do not hinder perceived representational validity and associated learning? This study was designed to answer these questions.

\subsubsection{Subject matter}

There is a dilemma between a modeler's wish to quantify and formalize a concept and the perception of the modeled phenomena held by other people. This dilemma becomes even more critical in an educational context. Although quantification often yields important insights into the structure and dynamics of a problem (Sterman, 2002), players of the game can be distracted by such quantification. Players can suffer from information overload caused by too much detailed information and they can be confused by the dynamic complexity. Effects of this can be: being confounded by ambiguous variables, misperceptions of feedback, flawed cognitive maps of causal relations or erroneous inferences about dynamics (Sterman, 1994). Researchers argue (Wolfe \& Gold, 2007; Hatzipanagos, 1995) that the complexity of a game or a simulation model affects learning. "A number of factors govern the effectiveness of simulations in achieving learning objectives. These include the complexity of a game [...] and its acceptance as a valid representation of the real world" (Moizer, Lean, Towler \& Smith, 2006, p. 50). The complexity of a model is regarded in the modeling and simulation literature as being related to the cognitive aspect, that is, the difficulty of understanding the modeled system in relation to the number of parts and elements that it contains (Chwif, Barretto \& Paul, 2000). We believe that this duality originates from considering the model's complexity from two different points of view: the users' point of view and the modelers' point of view. Regarding simulation games, Wolfe (1985) listed several complexity factors which influence the effectiveness of a game: "the player's manual, the number of decisions programmed, the internal algorithms employed, and the number of supplemental materials and reports generated by the game" (Wolfe, 1985, p. 275). From these, the 
number of decisions programmed and the internal algorithms employed are factors which clearly reflect a model's complexity. In addition, these two factors affect learner/player behavior in the game to a different extent and in a different manner: the first one directly and the latter indirectly.

From the modelers' point of view, a model's complexity is related to the notion of the model's fidelity and the model's representational validity since the modelling of a phenomenon can be done at different levels of abstraction and precision.

In this research we consider the model's complexity from a cognitive perspective (point of view of the user of a model) and as a combination of two factors which affects learner/player behavior in the game directly: the number of observable variables and the number of decisions to be taken by players in the game. These will likely influence the perceived representational validity of the model and the model's educational validity.

Wolfe and Jackson (1989) reported that only a few studies have been conducted on the interaction between a game's complexity and its objectively assessed learning effect while no research has been done on the relationship between a game's degree of realism and the amount of learning that accompanies such realism. In this study we cover the latter issue. By including or excluding an explicit representation of knowledge process related variables for players/learners, we vary the model's complexity while keeping the behavior of the internal algorithm unchanged and as a consequence we vary the physical and functional fidelity presented to the learners. By doing this we affect the perceived degree of realism or perceived representational validity and consequently we will assess differences in learning that could occur due to this variation.

In other words, by quantifying knowledge and knowledge processes and by including knowledge process variables in the model, we increase the model's complexity, decrease physical fidelity (what exists in reality) and increase functional fidelity (how it behaves) (see section 4.2.2.2) of the model and modify the representational validity of the model since the variables are not known to and accessible for learners.

Nonetheless, we believe that the presence of knowledge process variables is crucial for understanding how knowledge management activities influence the knowledge household of a company; therefore, they are needed in the model. To be precise, the knowledge process related variables are unknown and unfamiliar to the players of the game, but those variables are incorporated into the model to show the dynamics and efficiency of knowledge processes, which influence the success of organizational processes and business outcomes.

In this experimental study we will investigate whether our expectations are correct or whether the formalization and quantification of knowledge processes negatively influences educational validity. Moreover, we will investigate what kind of relation exists between the perceived representational and educational validity of the model. Knowing these relations could give us an opportunity to optimize the educational validity of the game. If the perceived representational validity of the model has a direct influence on the educational validity of the model, we can increase the educational validity of the model and the entire game by changing the properties of the model; for example, by making the model transparent or by changing the model, the possibilities to increase the game's 
educational validity could probably be found in the game environment (i.e., additional player support).

\subsubsection{Research questions and expected outcomes}

In Chapter 4 we stated the hypothesis that if there is no representational validity, the model will not be educationally valid. In this experiment we take as a given that the model possesses at least some representational validity and we will investigate the relationships between different levels of fidelity in the model and the perceived representational validity and educational validity.

Usually decisions about the fidelity of a model are made in the beginning of the modeling process and later model developers assess the representational validity of the model with experts or with users of the model. Experimentally, we can create an environment in which we can vary the level of fidelity of the model for users of the model by presenting or hiding knowledge process related variables from the players. The model has the same behavior and the same relationships are present, but it will be shown differently to the players. One representation (Condition 1) will include knowledge process related variables and another representation (Condition 2) will exclude these variables. Referring to our conceptual model (see Fig 2.3, p. 24), in one representation all variables will be shown to the players (that is, all model layers) and in another representation only three layers of the model, excluding the bottom layer, will be shown. Thus, artificially we create conditions of the model with different fidelity levels for the players of the game (see Table 5.1). In Table 5.1 an overview of two versions of the model and our expectations associated with these versions are presented.

Table 5.1 Overview of model's fidelity for two experimental conditions and expectations

\begin{tabular}{|l|l|l|l|l|}
\hline & \multicolumn{2}{|c|}{ Fidelity } & \multicolumn{2}{c|}{ Expectations } \\
\hline & Physical & Functional & $\begin{array}{l}\text { Perceived } \\
\text { external and } \\
\text { internal } \\
\text { representational } \\
\text { validity }\end{array}$ & $\begin{array}{l}\text { Internal and } \\
\text { external } \\
\text { educational } \\
\text { validity }\end{array}$ \\
\hline $\begin{array}{l}\text { Condition1 (higher } \\
\text { complexity since } \\
\text { knowledge process } \\
\text { variables are included) }\end{array}$ & lower & higher & lower & higher \\
\hline $\begin{array}{l}\text { Condition2 (lower } \\
\text { complexity - } \\
\text { knowledge process } \\
\text { variables are } \\
\text { excluded) }\end{array}$ & higher & lower & higher & lower \\
\hline
\end{tabular}

We will investigate whether different levels of physical and functional fidelity influence the representational and educational validity of the model and what kinds of relationships between perceived representational validity and educational validity of the model exist in the case of modeling an abstract and unfamiliar phenomenon. Does the 
model need to possess a high physical or functional fidelity to be educationally valid or does the model need to possess both physical and functional fidelity at a high level for the effective teaching of such an abstract concept?

The first two columns of Table 5.1 characterize the two conditions in terms high or low physical and functional fidelity. The third and fourth columns represent the expected effects of each condition (in terms of fidelity levels) on perceived external and internal representational validity and internal and external educational validity.

We expect that a higher level of functional fidelity and a lower level of physical fidelity will increase internal and external educational validity since players have to learn "important structures and behavior modes" (Maier \& Größler, 2000, p. 136); in other words, players have to learn how to operate in the system and not how to operate $a$ system. They do not need precise procedural knowledge, but they have to develop insights as to how a system behaves under different conditions and predict the behavior of the system. At the same time, a higher level of functional fidelity and a lower level of physical fidelity will decrease perceived representational validity since the model will include unknown variables. In the opposite case, a lower level of functional fidelity and a higher level of physical fidelity will (1) decrease the internal and external educational validity of the model since players could have difficulties connecting knowledge management activities (that is, knowledge management interventions) and the knowledge of a company to the company's organizational outcomes and at the same time will (2) increase perceived internal and external representational validities since the model includes familiar variables used in economics and business monitoring.

In a more formal fashion, we hypothesize the following:

H1: The model including knowledge process related variables (Condition 1) will lead to a higher internal educational validity than the model excluding knowledge process related variables (Condition 2).

H2: The model including knowledge process related variables (Condition 1) will lead to a higher external educational validity than the model excluding knowledge process related variables (Condition 2).

H3: Participants in Condition 1 will perceive the external representational validity of the model lower than participants in Condition 2 will perceive the same validity.

H4: Participants in Condition 1 will perceive the internal representational validity of the model lower than participants in Condition 2 will perceive the same validity.

In other words, participants in Condition 1 will judge the model as being less representatively valid when compared to the participants in Condition 2, because knowledge processes and variables describing these processes are unfamiliar to them. At the same time participants in Condition 1 will show better learning results when compared to participants in Condition 2, because these processes and variables are important for learning how the knowledge household of a company influences the organizational outcomes of a company, thus leading to a higher educational validity of the model.

\subsection{Method}

\subsubsection{Design and subjects}


This study lies at the interface of science and design (Klabbers, 2006). It focuses on an experimental design which involves "action experiments in authentic educational settings [...] rather than objective experiments with control groups" (Romme, 2004, p. 429). In this experimental study we used a post-test design (Krathwohl, 1988) to eliminate differences between the two conditions (see 5.1.3) when testing the perceived representational and educational validity of the model.

Subjects in the study were 52 third-year students from the University of Twente, Faculty of Behavioral Sciences who were in the final phase of the course "Knowledge management in learning organisations". They all followed the same curriculum, therefore we consider their prior knowledge about knowledge management to be approximately equal. Subjects were randomly assigned to one of the conditions without knowing the differences between the game versions. One group played the game with the model, which presented to the players the knowledge process related variables (Condition 1), and another group played the game without the opportunity to observe these variables (Condition 2). Participation in the experiment was voluntarily but could bring points for the final mark for the course, therefore the subjects were eager to participate and work seriously. Both participation itself and the quality of participation were counted as points for the final mark: success in the game and correct answers on the post test brought more points than poor performance in the game and incorrect answers on the test. The game session for subjects in Condition 1 was conducted four days earlier then the game session for subjects in Condition 2.

\subsubsection{Criterion measures}

\subsubsection{Educational validity of the model}

In our study to test which model condition provides higher external and internal educational validity we have to rely on our validity definitions given in Section 4.3. There we stated that the model is internally educationally valid if players of the game successfully use and develop conceptual knowledge in the knowledge management domain. This was defined by an ability to discern relationships between knowledge management bottlenecks, knowledge management interventions and organizational outcomes and the ability to address these relationships in the intended manner. The model is externally educationally valid if players are able to develop strategic knowledge, that is, master decision-making skills in the knowledge management domain. Thus, for assessing which version of the model presented to the players leads to higher internal and external educational validities, we have to define measures that help us to evaluate the game performance of the players (as a measure of addressing knowledge management relationships in the intended manner) and help us to evaluate the conceptual and strategic knowledge of players (as measures of understanding the phenomenon and the development of decision-making skills in the knowledge management domain).

When educators evaluate the learning effectiveness of a simulation, they most often use knowledge tests (Größler, 2001; Thomas \& Hooper, 1991, Alessi \& Trollip, 1991). Thomas and Hooper (1991) argue that most knowledge tests that are used to measure the effects of simulations measure the wrong thing. Tests should measure transfer and application instead of measuring the ability to recall information that is typical of many 
knowledge tests. "For example, if the students were given an instructional simulation which was designed to teach them problem-solving skills, the best test is to give them a variety of problems related to but not the same as the ones in the instructional lesson. In this way you assess whether what was taught has transferred to the situations for which the skill was needed" (Alessi \& Trollip, 1991, p. 382). An advantage of tests measuring "transfer and application" is, as pointed out by Größler (2001), their ability to assess the qualitative goal of knowledge accumulation - changes in cognitive structures. Following this line we will use a knowledge test which measures not only the conceptual knowledge gained in the game but also the transfer of knowledge, that is, strategic knowledge.

In our study we will assess internal educational validity by measuring the performance of the players in the game and achievement on the knowledge test assessing players conceptual knowledge. That is, players' understanding of the modeled phenomenon and the model's relationships, particularly how knowledge management activities influence the state of knowledge in the company. External educational validity will be measured by the achievement on a test assessing players' strategic knowledge or, in other words, players' ability to solve problems in the knowledge management domain which differ from the ones they encountered in the game.

Researchers and practitioners in the field of gaming research use different measures of game results, including performance results such as firm earnings and profits (Wolfe \& Chacko, 1982; Washbush \& Gosen, 2001), learning outcomes and learning sources (O'Neil, Wainess \& Baker, 2005) and decision-making behaviors and decision-making time (Kocher \& Sutter, 2007). Every study and every game is different and there are no commonly used measures of game results. In this study, in order to be able to compare performance in the game we created and used several measures. One can argue that these measures are not unambiguously valid, but in our view they suit our goal - they can reveal differences in performance between players in the two conditions:

- Company Profit - an internal game indicator. Students in Condition 2 can not observe changes in the knowledge process related variables (including their decay behavior) and can not make inferences about the effects of knowledge management interventions on these variables, which makes achieving a high level and quality of knowledge in the company and of good business outcomes in the game more difficult. They have to orient in the system based on the scores of business process related indicators and organizational performance indicators. One of these is Profit, which is the most commonly used indicator of company success. Thus this indicator assesses students' success in playing, without favoring one of the conditions, as this indicator is accessible in both conditions. In line with our $\mathrm{H} 1$, we expect that players in Condition 1 will have higher scores on this indicator, because they will orientate in the system also based on the observation of knowledge process variables and not only based on information about events and other indicators on which players in Condition 2 have to rely.

- K-value - an indicator assessing the level of knowledge in the company. In contrast to the profit indicator, this indicator reveals if students pay attention to the level of knowledge in the company which can be directly influenced by 
knowledge management interventions. If students paid attention to knowledge processes, we expect that students in Condition 1 will show better results on this indicator, which will support our H1. They could observe knowledge process variables which contribute directly to the variables "Level of competence" in each knowledge domain, the sum of which constitutes the K-value indicator. This option was not available to players in Condition 2 as they lacked the information about the knowledge process variables.

- Number of interventions chosen by a player $(\mathrm{N})$. This indicator reveals how effectively students selected their interventions based on the information in the game: events, interventions and values of observable indicators. Again, according to $\mathrm{H} 1$, we expect that students in Condition 1 will implement fewer interventions than players in Condition 2, because they could observe knowledge process related variables and therefore choose better fitting interventions from the predefined list of interventions. These better fitting interventions would lead to better results, which in turn would reduce the need to take more interventions.

The game is designed in such a way that knowledge, measured as the level of competence in all knowledge domains, declines over time, because knowledge process related variables exhibit decay behavior. To maintain knowledge on a high level, players of the game should implement interventions for all knowledge domains in each game period. At the same time, to deal with or propagate the effects of events that occur in the game, players have to implement interventions. Taking into account the decline of the knowledge level in three knowledge domains and the effects of game events, players should implement up to 4 or 5 interventions in each game period: one intervention to counter the decline of knowledge for each knowledge domain (there are three in the game, see sec. 2.3.1) and one or two interventions to react upon the game event.

The knowledge test used in this study was developed to measure conceptual knowledge (internal educational validity) and strategic knowledge (external educational validity). The What-if test (Swaak, 1998) and the HollandSky transfer test (Christoph et al., 2003; Leemkuil, 2006) contributed to the development of the knowledge test. The test items were explicitly based on the learning goals for the acquisition of conceptual and strategic knowledge formulated in KM Quest (Leemkuil et al., 2002, p.14), where learners:

- Are able to recognize a knowledge management problem or opportunity;

- Are able to relate knowledge management work to business results through established performance indicators;

- Are able to assess the knowledge management situation and advise/implement appropriate interventions;

- Are able to monitor and evaluate the consequences of interventions.

Hence, conceptual knowledge in this study refers to the internal educational validity and is defined as the ability of players to discern the relationships of the modeled phenomena. In other words, players should know the interventions' influences on the knowledge variables of the model. Strategic knowledge in the game refers to the external educational validity and is defined as the ability of players to recognize a knowledge 
management problem and solve the problem by selecting appropriate knowledge management interventions. Strategic knowledge is based on conceptual knowledge.

In the test for measuring conceptual knowledge, we offered students 10 knowledge management interventions (from those which were used in the game) and asked them to specify the influence of each of the interventions in terms of the affected knowledge domains and type of influence - delayed or immediate. When evaluating the answers, we compared students' specifications with the interventions' specifications used in the model. These are taken as the standard because players of the game construct their conceptual knowledge based on the general behavior of the game that is driven by the specifications in the model. Each intervention was assessed on 4 levels: 0 points - no correct answers; 0.1 points - few domains mentioned without influence; 0.2 points - all domains mentioned without influence or few domains mentioned with correct influences; 0.3 points - all domains mentioned with correct influences. In total this part of the test consisted of 10 items and could bring students 3 points.

For measuring strategic knowledge, we offered students a description of 5 different events that occur in the same company as in the game (Coltec), but that did not occur in the game they played. For each event, there is one multiple choice question about the nature of the event, whether it is a problem event, opportunity event or neither, and two questions that require an open-ended answer. The first question asks students to write down consequences of this event for the company and the second question asks students to select knowledge management interventions from a predefined list that will either, in their judgment, prevent the effect of the event or propagate its effect. In total this part of the test consisted of 15 questions, 3 questions for each event. For evaluating the answers, we asked two experts to complete this part of the test in order to compare their decisions with the students' decisions. We used this information to evaluate the students' answers. Decisions of the experts were taken as the standard against which students were assessed. If students' answers matched the answer of at least one of the experts, his or her answer was counted as correct. For each event in the first question students could score 0.2 points for the right answer, 0.1 points if they choose one alternative when 2 were possible, and 0 points - for an incorrect answer. On each of the open-ended questions students could score 0.4 points for a correct answer, 0.2 points if they did not mention all consequences of an event or did not select interventions to react on all consequences of the event and 0 points for an incorrect answer. Overall students could earn a maximum of one point for each event for a total of five total points for the five events. In accordance with hypotheses $\mathrm{H} 1$ and $\mathrm{H} 2$, we expected that students in Condition 1 will score better on both parts of the knowledge test. This follows from our idea that knowledge process variables are important for learning how knowledge and the dynamics of knowledge processes influence organizational outcomes.

Examples of interventions and event used in test and their associated items can be found in Appendices 5 and 6.

\subsubsection{Perceived representational validity of the model}

In Chapter 4 we stated that if the model provides a plausible representation of relationships between knowledge, knowledge management interventions, events and 
organizational performance, the model has internal representational validity. To possess external representational validity the model needs to resemble actual organizational situations.

A game's degree of realism, or representational validity, is assessed differently in simulation studies. Wolfe (1989, p. 277), based on the work of Dukes and Waller (1976), mentioned three components influencing a game's subjectively assessed level of realism: accuracy - the degree to which a game represents the model of reality that it purports to describe; plausibility - the degree to which a game takes cognizance of experience and capabilities of the players; and relevance - the degree to which a game relates to the concerns of the players. Feinstein and Cannon (2001) list three constructs of perceived validity: verisimilitude, which refers to the representation of real-life phenomena; plausibility, which refers to the ability to represent real-life phenomena; and believability, which refers to users' confidence in the model's results. Couture (2004) also refers to terms like possibility, plausibility and existence. As several authors propose different terms and definitions, there is no agreed upon way to deal with the notion of representational validity.

In this study we borrow some of the definitions described above. We will assess perceived representational validity as a combination of three categories, which have a different degree of relevance for representational validity:

- Plausibility - the model and its components are regarded as adequate to learn about inferences in the game;

- Applicability- the model and its components could be used to perform knowledge management-like work in actual companies

- Credibility - the model and its components represent relevant and actual features of real product leadership companies.

Perceived internal representational validity is assessed with the Plausibility category subjects have to evaluate model behavior and its components in the game as being plausible for learning relationships between knowledge, knowledge management interventions and organizational outcomes. Perceived external representational validity is assessed with the Applicability and Credibility categories - subjects have to evaluate the model and its components as being applicable and relevant to reality.

To measure perceived internal and external representational validity of the simulation model, we created a validity questionnaire that had to be answered by the students (Appendix 7). The validity questionnaire contained two parts. The first part explains the conceptual model, which is 'hidden' behind the indicators and not visible for the players. The second part consists of 33 items concerning propositions about the conceptual model in general and the 10 components of this model. Each component had to be judged on the 3 representational validity categories:

1. Conceptual model

2. Knowledge domains

3. Knowledge processes

4. The business process variables

5. The knowledge processes related variables

6. The knowledge management interventions

7. The events 

8. The decay behavior of the model
9. The effects of interventions
10. The effects of events
11. The overall behavior of the model.

The first proposition refers to the Plausibility of the model or its components (propositions 1, 4, 7, 10, 13, 16, 19, 22, 25, 28, 31); the second proposition refers to the Applicability of the model or its components (propositions 2, 5, 8, 11, 14, 17, 20, 23, 26, $29,32)$; and the third proposition refers to the Credibility of the model or its components (propositions 3, 6, 9, 12, 15, 18, 21, 24, 27, 30, 33). See Example 5.1 below.

Example 5.1. Plausibility, applicability and credibility propositions for the knowledge process variables in the validity questionnaire

The knowledge process related variables are adequate for monitoring performance of the company (Coltec) in the game (Plausibility proposition).

The knowledge process related variables can be used by other companies for monitoring their performance (Applicability proposition).

The knowledge process related variables are realistic to be used by product leadership companies for monitoring their performance. (Credibility proposition).

The propositions are rated on a six-point scale, ranging from strongly disagree to strongly agree. The questionnaire was used also within the KITS project for the second prototype evaluation (Christoph et al., 2003) and its content was discussed and evaluated with partners of the project.

In line with our $\mathrm{H} 3$ and $\mathrm{H} 4$ we expect that players in Condition 1 will judge the model as being less internally and externally representatively valid when compared to judgments of players from Condition 2, because the model includes variables unknown to condition 1 players which makes it harder for them to link the model to what they already know.

\subsubsection{KM Quest environment in the experiment}

To prevent differences between conditions and associated game performance and learning, we also changed the game environment. We excluded visualization features, advice features, and the description of the knowledge process related variables. Additionally we changed the description of interventions for both conditions in such a way that they did not provide information about the model's internal influences and inferences. We also closed access to those parts of the supportive knowledge management model where players could focus on the knowledge process related variables. In this way we severed the link between the supportive KM model and conceptual knowledge (see Figure 4.2). By doing this, players in both conditions did not receive guidance in the process of acquiring conceptual knowledge. Therefore we could associate the differences in game performance and differences on the test scores with the presence of the knowledge process related variables in the model only, and not with the 
game environment which normally (with available supportive normative model) supports players in their process of finding knowledge management bottlenecks and selecting the right interventions. From the game features, a feedback function was available to the players in both conditions. After each game period, the players of the game could get information about the event which occurred in the previous game period together with a list of possible interventions which should be taken to counter or propagate the effect(s) of the event.

In both conditions the underlying behavior of the model was the same; applying the same intervention would lead to the same changes in the values of variables, with the only difference that students in Condition 2 could not see the knowledge process related variables.

In the experiment students had to play 8 game quarters. We choose 8 game periods because this duration is sufficient to see the delayed effect of interventions and the overall behavior of the model. All games had the same initial values for all variables and the same sequence of events. This was done to guarantee that the initial conditions were the same for every subject. Otherwise different playing conditions and differences in the game environment could influence game performance and the test results.

Performance in the game was recorded in the log files. From these log files we could see when a player was logged onto the game, the duration of the session and what he or she had done. For our criterion measures based on game performance, we relied on the following data from the log files: the values of the indicators in the game after each game period, the game budget and the selection of interventions by a player in each game period.

\subsubsection{Procedure}

At the start of the course "Knowledge management in learning organizations" subjects were informed about the opportunity to participate in the experiment. They were informed that their performance in the game and their test results could bring additional points for the final mark for the course. Additionally, the three best players, based on their performance in the game in both groups, would be rewarded with small gifts. Students could enroll for playing the game on one of two days. They were not informed about the differences in the game versions and their being assigned to one of the different conditions. The experiment took place half-way through the course.

For both groups the session began with an introductory explanation by an instructor. The game environment was explained as well as the rules that would be applied for selecting the best game performance. They included: playing the game and staying within the game budget and having the highest values on organizational performance indicators and on the knowledge level indicators. It was explained that applying a large number of interventions in one game period would not work well, because in real life companies do not make many changes at the same time. Furthermore, it was pointed out that knowledge can decrease over time and, as a consequence, players have to pay attention to all knowledge areas in their company. Students were informed that they had to play the game individually and that each of them had to play his or her own game. They were informed that they do not "fight" against another player while playing, but compete 
against the game environment. They were informed that they have the same starting values of the variables and that their games will provide the same sequence of game events, giving everyone an equal chance to perform well. They were informed about things that would be absent in the game environment: visualization, collaboration, and advice options as well as being informed that some links do not have additional information. After this explanation, students got access to the game with an individual login and password. The games were played via Internet. Students were asked to play at least 4 game periods during the session and the remaining game periods in their spare time within two weeks after the session. After playing 4 game periods individually in the session, it was assumed that they would all be in different game situations, because they applied different interventions during these game periods. This made it less likely that the students would or could support each other in playing the game in the four game periods played outside the fixed sessions. Collaboration was probably even less likely due to the competitive character of playing the game.

After two weeks (which were used to finish playing the game) students from both groups were invited to a two-hour session to complete the paper-based knowledge test and fill in the validity questionnaire. Before answering the test, all subjects received a standard description of the knowledge processes which were modeled in the game. This was done to ensure that subjects from Condition 2 could answer test questions. Therefore these subjects were familiar with the meaning of knowledge processes, but they could not observe variables formalizing these processes while playing the game. The test did not include questions about exact relationships between variables. One subject from Condition 2 did not finish playing the game and therefore was not allowed to participate in the test. After two weeks the results of the game performance were announced and tests were presented to the students during a regular course session.

\subsection{Results}

The log files reveal that several players in both conditions overspent the game budget because they applied a large number of interventions. Their game performance data, test data and judgments on representational validity were excluded from the analysis, because these players did not play within the set rules. Results of 6 subjects from Condition 1 and 5 subjects from Condition 2 were excluded from the analysis.

\subsubsection{Educational validity of the model}

Internal educational validity: performance in the game

Performance in the game was assessed using three performance measures: Profit indicator - cumulative profit for each game at the end of the game, K-value indicator - sum of the final values of the Level of competence indicators for three knowledge domains and $\mathrm{N}$ indicator - the number of interventions taken in the game. A good performance in the game means to achieve higher values on the Profit and $\mathrm{K}$ indicators and apply fewer interventions when compared with the standard (see section 5.2.2.1). The standard in this case is 4-5 interventions per game period, which leads to 32-40 interventions for 8 periods. 
Table 5.2 shows the mean values (and standard deviations) of these measures for both conditions.

Table 5.2 Mean values and standard deviations of three performance measures for the two conditions

\begin{tabular}{|l|l|l|l|l|}
\hline Measure & Condition & N & Mean & St. deviation \\
\hline \multirow{3}{*}{ Profit } & Condition 1 & 20 & 72480905.70 & 15362168.058 \\
\cline { 2 - 5 } & Condition 2 & 20 & 67026065.40 & 12551925.428 \\
\hline $\mathbf{N}$ & Condition 1 & 20 & 43.75 & 8.403 \\
\cline { 2 - 5 } & Condition 2 & 20 & 41.80 & 9.260 \\
\hline \multirow{2}{*}{ Condition 1 } & 20 & 24.0350 & 2.83432 \\
\cline { 2 - 5 } & Condition 2 & 20 & 21.1065 & 2.45411 \\
\hline
\end{tabular}

A t-test for independent samples shows that there are no significant differences in game performance between players in the two conditions on the Profit and $\mathrm{N}$ indicators ( $>>0.05)$, although the mean scores on these indicators show that players in Condition 1 have slightly higher values than the mean scores on the same indicators for the players in Condition 2. At the same time, the mean values on the $\mathrm{K}$-indicator for players in Condition 1 are significantly higher than the mean values on the K-indicator for players in Condition $2(\mathrm{t}(38)=3.493, \mathrm{p}<.01)$. From these results, we conclude that $\mathrm{H} 1$ is only partially supported. The higher values of the $\mathrm{K}$ indicator in Condition 1 could indicate that players in Condition 1 paid attention to the knowledge process variables they had access to. As the level of the K-indicators depend directly on the values of these knowledge process variables; not having access to the latter (Condition 2) will probably lead to lower scores on the former.

Concerning the $\mathrm{N}$ indicator, we can see that players in both conditions applied slightly more (42 and 44) interventions than were necessary for optimal playing. The absence of a difference between the two conditions on the $\mathrm{N}$ indicator can explain why there is no difference on the Profit indicator. Each intervention in the game individually consists of some expenses which are subtracted from the company Profit when the intervention has been implemented. For example, the training program could cost the company 20.000 or 40.000 euro depending on which training program is carried out in the company. These costs are subtracted from the company Profit. In the game there are also costs which are associated with the process of choosing interventions before incurring the additional costs of implementing them in the company. This served as a vehicle to force players to choose better fitting interventions and not to implement all types of interventions in one game period. The costs for interventions are subtracted from the game budget. Thus costs for choosing an intervention in the game are different from expenses associated with implementing the intervention in the company. For example, costs for choosing the training interventions could be the same and will be subtracted from the game budget, but the effect of the interventions on the company expenses as well as on the knowledge processes and domains are different. 
Internal and external educational validities: knowledge test

The knowledge test was administered in order to find out which model representation provides higher internal and external educational validity. We measured conceptual knowledge (as the indicator for internal educational validity) and strategic knowledge (as the indicator for external educational validity) of players in the two conditions. Cronbach's $\alpha$ for the part of the test measuring conceptual knowledge yielded 0.773 and the part measuring strategic knowledge yielded 0.725 , which is satisfactory.

Table 5.4 shows the means and standard deviations on the knowledge test for both conditions.

Table 5.4 Means and standard deviations of the knowledge test scores for both conditions

\begin{tabular}{|l|l|l|l|l|}
\hline $\begin{array}{l}\text { Type of } \\
\text { knowledge }\end{array}$ & Condition & N & Mean & $\begin{array}{l}\text { Standard } \\
\text { deviation }\end{array}$ \\
\hline Conceptual & Condition 1 & 20 & 1.950 & .4915 \\
\cline { 2 - 5 } & Condition 2 & 20 & 1.545 & .6151 \\
\hline \multirow{2}{*}{ Strategic } & Condition 1 & 20 & 4.280 & .5105 \\
\cline { 2 - 5 } & Condition 2 & 20 & 3.650 & .9202 \\
\hline
\end{tabular}

The achievement on the knowledge test was analyzed with a t-test for independent samples. Results of the knowledge test reveal that players in Condition 1 scored better on items assessing conceptual knowledge $(\mathrm{t}(38)=2.300, \mathrm{p}=.027)$ and on items assessing strategic knowledge $(\mathrm{t}(29.7)=2.677, \mathrm{p}=.012)$. This confirms that a model providing information about knowledge process related variables, that is, a model possessing higher functional fidelity and lower physical fidelity, provides higher internal and external educational validity than a model that does not give this information. $\mathrm{H} 1$ and $\mathrm{H} 2$ are supported.

\subsubsection{Perceived representational validity of the model}

The perceived representational validity of the model was assessed for players in both conditions using the validity questionnaire. The reliability of the entire questionnaire equaled a Cronbach's $\alpha$ of 0.9173 . The reliability of the part assessing plausibility items yielded 0.800 , the applicability part 0.824 and the credibility part 0.785 , which are satisfactory.

To find the differences between the two groups with respect to perceived representational validity, we used a t-test for independent samples. The mean judgment scores and standard deviations for both conditions are displayed in Table 5.5. 
Table 5.5 Mean judgment scores for the perceived representational validity of the model for both groups

\begin{tabular}{|c|c|c|c|c|c|}
\hline Model components & \begin{tabular}{|l|} 
Condition \\
\end{tabular} & $\mathrm{N}$ & Plausibility & Applicability & Credibility \\
\hline \multirow[b]{2}{*}{ Conceptual model } & Condition 1 & 20 & $4.20(1.056)$ & $4.55(.945)$ & $3.90(.788)$ \\
\hline & Condition 2 & 20 & $4.50(1.147)$ & $4.85(.587)$ & $4.50(607)$ \\
\hline \multirow[b]{2}{*}{ Knowledge domains } & Condition 1 & 20 & $4.55(.887)$ & $4.45(.826)$ & $4.65(.988)$ \\
\hline & Condition 2 & 20 & $4.40(1.095)$ & $4.35(.745)$ & $4.45(.686)$ \\
\hline \multirow[b]{2}{*}{ Knowledge processes } & Condition 1 & 20 & $3.90(1.210)$ & $4.45(.605)$ & $4.50(1.00)$ \\
\hline & Condition 2 & 20 & $4.35(1.040)$ & & $4.50(.761)$ \\
\hline \multirow{2}{*}{$\begin{array}{l}\text { The business process } \\
\text { variables }\end{array}$} & Condition 1 & 20 & $4.60(.9$ & $4.65(.9$ & 75) \\
\hline & Condition 2 & 20 & $4.70(1.031)$ & $4.75(.851)$ & $4.65(.875)$ \\
\hline \multirow{2}{*}{$\begin{array}{l}\text { The knowledge process } \\
\text { related variables }\end{array}$} & Condition 1 & 20 & & $3.75(1$. & $3.55(.945)$ \\
\hline & Condition 2 & 20 & & $4.65(.875)$ & $4.50(.827)$ \\
\hline \multirow[b]{2}{*}{ The interventions } & Condition 1 & 20 & & 1) & 301) \\
\hline & Condition 2 & 20 & 4.7 & 4.7 & .182) \\
\hline \multirow[b]{2}{*}{ The events } & Condition 1 & 20 & 4.3 & 4.45 & 889) \\
\hline & Condition 2 & 20 & 4.45 & 910) & 33) \\
\hline \multirow{2}{*}{$\begin{array}{l}\text { The decay behavior of } \\
\text { the model }\end{array}$} & Condition 1 & 20 & 4.15 & 4.7 & 4.7 \\
\hline & Condition 2 & 20 & 4.80 & $5.00(.725)$ & 4.7 \\
\hline \multirow{2}{*}{$\begin{array}{l}\text { The influence of } \\
\text { interventions }\end{array}$} & Condition 1 & 20 & $3.40(1.392)$ & $4.30(.657)$ & $3.85(.813)$ \\
\hline & Condition 2 & 20 & $4.10(1.165)$ & $4.10(.968)$ & $4.00(1.124)$ \\
\hline \multirow[b]{2}{*}{ The influence of events } & Condition 1 & 20 & $4.05(1.234)$ & $4.15(.745)$ & $3.90(1.021)$ \\
\hline & Condition 2 & 20 & $4.10(1.518)$ & $4.60(.995)$ & $4.05(.945)$ \\
\hline \multirow{2}{*}{$\begin{array}{l}\text { The behavior of the } \\
\text { model }\end{array}$} & Condition 1 & 20 & $4.15(.875)$ & $4.15(.745)$ & $4.00(.858)$ \\
\hline & Condition 2 & 20 & $4.10(1.021)$ & $4.35(.813)$ & $4.25(.851)$ \\
\hline \multirow[b]{2}{*}{ Aggregation } & Condition 1 & 20 & $45.60(5.413)$ & $46.40(5.051)$ & $47.80(5.634)$ \\
\hline & Condition 2 & 20 & $48.55(8.127)$ & $48.30(5.686)$ & $50.75(5.514)$ \\
\hline
\end{tabular}

At the aggregate level, which is the sum of all validity propositions for the plausibility, applicability and credibility categories, we did not find significant differences between judgments of players in the two conditions, although players in Condition 2 tended to judge perceived representational validity more positively. This means that the presence of knowledge process related variables (the unfamiliar variables) does not influence representational validity of the model. H3 and $\mathrm{H} 4$ are rejected. Detailed analysis and a comparison of judgments for all validity propositions, revealed that students in Condition 2 judged the game simulation model more positively on most validity propositions than did students in Condition 1 . The mean scores of all players in Condition 2 for all propositions is higher than 4. Moreover, players in Condition 2 also judged positively the propositions which they could not judge based on playing the game - propositions related to the knowledge process related variables. This might be caused by the fact that all students could read about these variables in the explanatory part of the validity questionnaire.

Players from Condition 1 gave mean scores below 4 for two propositions assessing perceived internal representational validity (Bold, Italic in Table 5.5):

- plausibility of knowledge processes for learning knowledge management

- plausibility of the influence of interventions in the game 
As for perceived external representational validity, the following propositions were assessed with scores below 4 by subjects from Condition 1(Bold in Table 5.5):

- credibility of the conceptual model

- applicability of knowledge process related variables

- credibility of knowledge process related variables

- credibility of the influence of the interventions in reality

- credibility of the influence events in reality

It is in line with our expectation that knowledge process related variables reduce the perceived representational validity of the model, but significant differences were found only for 4 items (grey cells in the Table 5.5).

There were significant differences in scores between the two conditions on the propositions assessing the applicability $(\mathrm{t}(38)=-2.835, \mathrm{p}=.007)$, and credibility $(\mathrm{t}(38)=-$ $3.384, \mathrm{p}=.002$ ) of knowledge process related variables, the credibility of the conceptual model $(\mathrm{t}(38)=-2.698, \mathrm{p}=.010)$, and the plausibility of the decay behavior of the model $(t(38)=-2.323, p=0.026)$. For the other propositions there were no significant differences between the mean scores of players in the two conditions. Based on this data we can say that $\mathrm{H} 3$ and $\mathrm{H} 4$ are rejected.

For items which were judged significantly different, three refer to the perceived external representational validity and one refers to the perceived internal representational validity.

These outcomes jeopardize the model's perceived representational validity. If we would provide additional support to players in the game concerning the relationships between knowledge processes related variables and the influences of interventions and events on these variables, or if we would leave the supportive knowledge management model accessible, the unfamiliar variables would be probably more understandable by players in Condition 1 and this would probably lead to a different perception of the model's perceived representational validity. This raises the question of whether there are any relationships between performance in the game and the presence of knowledge process related variables and judgments on perceived representational validity. Good or bad performance in the game can influence players' feelings of satisfaction and could affect their judgments on the perceived representational validity. On the other hand, as some researchers believe (Moizer, Lean, Towler \& Smith, 2006), the acceptance of the game model as a valid representation might influence playing behavior and learning results. The next subsection investigates these relations.

\subsubsection{Relationships between perceived representational validity and educational validity}

In this section we investigate whether there are any relationships between performance in the game (which we defined as being indicative for internal educational validity) and judgments of the representational validity (which are measures of internal and external representational validity). At the same time we look at whether the acceptance of the model as a valid representation of reality is related to the knowledge test scores (which are measures of internal and external educational validity).

Performance in the game could affect validity judgments because subjects first played the game and then completed the questionnaire. Therefore we computed Pearson 
correlations between performance indicators and validity judgments for all subjects in order to see whether there were any correlations between performance and subjects' judgments of validity propositions.

In order to see whether the acceptance of the game model as a valid representation influences playing behavior and learning results we computed Pearson correlations between measures of educational validity and validity judgments. As the two conditions had different fidelity levels of the model, which can influence the perception of the model as a valid representation of reality and consequently educational validity, we computed the correlations for these conditions separately.

\subsubsection{Relationships between performance indicators in the game (internal educational validity) and perceived representational validity}

Results revealed that there are several significant negative correlations between the Profit and K-value performance indicators and the judgments on validity items. Students who achieved higher values on the Profit and K-value indicators (or in other words, who performed better in the game) judged the perceived representational validity of the model less positively. We did not find any correlations between the $\mathrm{N}$ indicator and validity judgments, which means that the players own behavior (in terms of the number of interventions selected) was not related to the validity judgments.

Of all significant correlations, only one is related to the Plausibility category, that is the perceived internal representational validity: players who achieved higher scores on the K-value indicator judged the plausibility of the effects of interventions less positively $(\mathrm{N}=40, \mathrm{r}=-.315, \mathrm{p}<.05)$. The other significant correlations were found between the Profit and $\mathrm{K}$-value performance indicators and judgments on the external representational validity items, which belong to the Applicability and Credibility validity categories.

Players who achieved higher values on the Profit performance indicator judged the following less positively than the other players: 1) the applicability of conceptual model, 2 ) the applicability of knowledge domains, 3) the applicability of knowledge processes, and 4$)$ the credibility of knowledge domains $(\mathrm{N}=40,1) \mathrm{r}=-.333, \mathrm{p}<.05,2) \mathrm{r}=-.316, \mathrm{p}<.05$, 3) $r=-.387, p<.05,4) r=-.327, p<.05)$.

Players, who achieved higher values on both Profit and K-value performance indicators judged the following less positively than did players who received lower values:

- the applicability of the knowledge process related variables $(\mathrm{r}=-.479, \mathrm{p}<.01$ and $\mathrm{r}=-.460, \mathrm{p}<.01$ respectively),

- the credibility of knowledge processes ( $\mathrm{r}=-.348, \mathrm{p}<.05$ and $\mathrm{r}=-.328, \mathrm{p}<.05$ respectively),

- the credibility of the knowledge process related variables $(\mathrm{r}=-.360, \mathrm{p}<.05$ and $\mathrm{r}=-$ $.447, \mathrm{p}<.01$ respectively), and

- the credibility of the effects of events $(\mathrm{r}=-.465, \mathrm{p}<.01$ and $\mathrm{r}=-.398, \mathrm{p}<05$ respectively).

These results indicate that better performance is related to less positive judgments about the model's perceived representational validity. An explanation for this finding could be that students who tried to achieve better results and played seriously thought more critically about the model, especially about its "real" applicability and "reality". 
5.3.3.2 Relationships between perceived representational validity and educational validity

To find out what kind of relationships exist in this experiment between the perceived representational and educational validities of the model and to answer the question of whether acceptance of the model as a valid representation influences the playing behavior and learning outcomes, we computed Pearson correlations for both conditions separately. One of the goals of this analysis was to investigate whether a common pattern in relationships between items assessing perceived representational validity, performance indicators and test scores exists for both groups. In case it exists, we might conclude that there are relationships between the perceived representational and educational validities which are not based on model properties or differences in the model. In the case that there is no common pattern, differences in judgments of the model are based on different fidelity levels of the model and the presence or absence in the model of knowledge process related variables. Consequently we can see how this influences the educational validity of the model. Do positive or negative judgments of the model as valid representation influence performance in the game and learning outcomes?

In order to find answers to these questions for both conditions, we computed Pearson correlations for the following combinations:

1. Perceived internal representational validity (plausibility items) and internal educational validity (performance indicators and test scores on conceptual knowledge)

2. Perceived internal representational validity (plausibility items) and external educational validity (test scores on strategic knowledge)

3. Perceived external representational validity (applicability and credibility items) and internal educational validity (performance indicators and test scores on conceptual knowledge)

4. Perceived external perceived representational validity (applicability and credibility items) and external educational validity (test scores on strategic knowledge).

We deal with each combination in turn.

From the three performance indicators, we used only two in the correlation analysis: the Profit and K-value performance indicators, because the Profit indicator and Levels of competence, which constitute the K-value indicator, could be observed directly by players. We left the $\mathrm{N}$-indicator out of the analysis because it was not observable by the players.

The relationships between the perceived internal representational validity and internal educational validity are shown in Table 5.6. 
Table 5.6 Relationships between perceived internal representational validity and internal educational validity

\begin{tabular}{|l|l|l|l|}
\hline \multirow{2}{*}{ Plausibility validity propositions } & \multicolumn{2}{|l|}{ Performance indicators } & Test scores \\
\cline { 2 - 4 } & Profit & K-value & $\begin{array}{l}\text { Conceptual } \\
\text { knowledge }\end{array}$ \\
\hline Condition 1 & & & \\
\hline Plausibility of conceptual model & $r=-.456 p<.05$ & & \\
\hline Plausibility of knowledge domains & & & $r=.549 p<.05$ \\
\hline & & \\
\hline Condition 2 & no correlations found & \\
\hline
\end{tabular}

From Table 5.6 it can be seen that players from Condition 1, who judged less positively the plausibility of the model achieved higher scores on the Profit indicator $(r=$ $-.456 p<.05)$ and those who judged more positively the plausibility of knowledge domains achieved higher scores on test items assessing conceptual knowledge $(r=.549 p<.05)$. These results show that the same pattern for both conditions does not exist and that there are no strong relationships between the perceived internal representational validity of the model and internal educational validity of the model, because we found only two correlations out of 33 possible (eleven plausibility validity propositions each multiplied by 2 performance indicators and the test score).

The relationships between perceived internal representational validity and external educational validity are shown in Table 5.7.

Table 5.7 Relationships between perceived internal representational validity and external educational validity

\begin{tabular}{|l|l|}
\hline Plausibility validity propositions & $\begin{array}{l}\text { Test scores } \\
\text { Strategic knowledge }\end{array}$ \\
\hline Condition 1 & no correlations found \\
\hline Condition 2 & \\
\hline Plausibility of conceptual model & $\mathrm{r}=.469 \mathrm{p}<.05$ \\
\hline Plausibility of knowledge domains & $\mathrm{r}=.491 \mathrm{p}<.05$ \\
\hline Plausibility of events & $\mathrm{r}=.507 \mathrm{p}<.05$ \\
\hline Plausibility of the model's behavior & $\mathrm{r}=.532 \mathrm{p}<.05$ \\
\hline
\end{tabular}

Results show that there is no significant correlation between judgment of the models' internal representational validity and external educational validity for players from Condition 1. Players from Condition 2, who assessed more positively the 
plausibility of the model, the plausibility of knowledge domains, the plausibility of events, and the plausibility of the model behavior, achieved higher scores on the test assessing strategic knowledge $(\mathrm{r}=.469 \mathrm{p}<.05, \mathrm{r}=.491 \mathrm{p}<.05, \mathrm{r}=.507 \mathrm{p}<.05$, and $\mathrm{r}=$ $.532 \mathrm{p}<.05$ respectively). The same pattern for both conditions does not exist and there is an indication that there is a positive relationship between perceived internal representational validity and external educational validity for players from Condition 2, players who could not observe knowledge process related variables.

The relationship between perceived external representational validity and internal educational validity can be found in Table 5.8.

Results (see Table 5.8) indicate that there are no common correlations between judgments of perceived external representational validity and internal educational validity for players from both conditions.

Table 5.8 Relationships between perceived external representational validity and internal educational validity

\begin{tabular}{|c|c|c|c|}
\hline \multirow{2}{*}{$\begin{array}{l}\text { Applicability and Credibility validity } \\
\text { propositions }\end{array}$} & \multicolumn{2}{|c|}{ Performance indicators } & \multirow{2}{*}{\begin{tabular}{|l} 
Test scores \\
$\begin{array}{l}\text { Conceptual } \\
\text { knowledge }\end{array}$ \\
\end{tabular}} \\
\hline & Profit & K-value & \\
\hline \multicolumn{4}{|l|}{ Condition 1} \\
\hline Applicability of knowledge processes & $\begin{array}{l}r=-.456 \\
p<.05\end{array}$ & & \\
\hline Applicability of the model behavior & & & $\begin{array}{l}r=.453 \\
p<.05\end{array}$ \\
\hline Credibility of knowledge domains & $\begin{array}{l}r=-.473 \\
p<.05\end{array}$ & & \\
\hline Credibility of knowledge processes & & $\begin{array}{l}r=-.457 \\
p<.05\end{array}$ & \\
\hline Credibility of events & $\begin{array}{l}r=.501 \\
p<.05\end{array}$ & $\begin{array}{l}r=.513 \\
p<.05\end{array}$ & \\
\hline Credibility of the effect of events & $\begin{array}{l}r=-.652 \\
p<.01\end{array}$ & $\begin{array}{l}r=-.447 \\
p<.05\end{array}$ & \\
\hline \multicolumn{4}{|l|}{ Condition 2} \\
\hline Applicability of knowledge domains & $\begin{array}{l}r=-.479 \\
p<.05\end{array}$ & & \\
\hline $\begin{array}{l}\text { Applicability of knowledge process related } \\
\text { variables }\end{array}$ & $\begin{array}{l}r=-.537 \\
p<.05\end{array}$ & $\begin{array}{l}r=-.492 \\
p<.05\end{array}$ & \\
\hline Credibility of knowledge process related variables & & $\begin{array}{l}r=-.578 \\
p<.01\end{array}$ & \\
\hline
\end{tabular}

In Table 5.8 for Condition 1 players there are five significant negative correlations between items of perceived external representational validity and the game indicators Profit and K-value, and two positive correlations between items of perceived external representational validity and Profit and K-value indicators. For players from Condition 2, 
four significant negative correlations between items of perceived external representational validity and Profit and K-indicators were found.

Players from Condition 1, who assessed less positively the applicability of knowledge processes, the credibility of knowledge domains and the credibility of the effect of interventions, achieved higher values on the Profit indicator than did players from the same condition who judged these aspects more positively $(r=-.456 p<.05, r=-$ $.473 \mathrm{p}<.05$, and $\mathrm{r}=-.652 \mathrm{p}<.01$ respectively). Players from Condition 1 , who perceived the credibility of knowledge processes and the credibility of the effect of interventions less positively, achieved higher values on the K-indicator $(r=-.457 \mathrm{p}<.05$ and $\mathrm{r}=-.447$ $\mathrm{p}<.05$ respectively). Players from Condition 1, who perceived more positively the credibility of the game events, achieved higher values on the Profit and K-value indicator $(\mathrm{r}=.501 \mathrm{p}<.05$ and $\mathrm{r}=.513 \mathrm{p}<.05$ respectively).

Players from Condition 2, who assessed less positively the applicability of knowledge domains and the applicability of knowledge process related variables (variables they could not observe in the game), achieved higher values on the Profit indicator $(\mathrm{r}=-.479 \mathrm{p}<.05$ and $\mathrm{r}=-.537 \mathrm{p}<.05$ respectively). Players from Condition 2, who judged the applicability of knowledge process related variables and the credibility of knowledge process related variables less positively achieved higher values on K-valueindicator $(\mathrm{r}=-.492 \mathrm{p}<.05$ and $\mathrm{r}=-.578 \mathrm{p}<.01$ respectively).

Only one significant correlation was found between items of perceived external educational validity and test scores assessing conceptual knowledge for players from Condition 1, and none were found for Players from Condition 2. Players from Condition 1 who assessed more positively the applicability of the model's behavior, achieved higher scores on the test items $(r=.453 p<.05)$.

From these results there is only a weak indication that players who achieved higher scores on the Profit and K-value indicators think more critically about the model, and there are no unidirectional and unequivocal relationships between perceived external representational validity and internal educational validity.

Finally, Table 5.9 shows the relationships between perceived external representational validity and external educational validity

Table 5.9 Relationships between perceived external representational validity and external educational validity

\begin{tabular}{|l|l|}
\hline Applicability and Credibility validity propositions & $\begin{array}{l}\text { Test scores } \\
\text { Strategic } \\
\text { knowledge }\end{array}$ \\
\hline Condition 1 & \\
\hline Applicability of the model behavior & $\mathrm{r}=.493 \mathrm{p}<.05$ \\
\hline Credibility of knowledge process related variables & $\mathrm{r}=.592 \mathrm{p}<.01$ \\
\hline Credibility of the model behavior & $\mathrm{r}=.480 \mathrm{p}<.05$ \\
\hline Condition 2 & $\mathrm{no}$ correlations found \\
\hline
\end{tabular}


Results in Table 5.9 show that there are significant positive correlations between perceived external representational validity and external educational validity only for players from Condition 1. Players who judged the applicability of the overall model behavior, the credibility of knowledge process related variables and the credibility of the overall model behavior, achieved higher scores on the test items assessing strategic knowledge. This indicates that there are positive relationships between perceived external representational validity and external educational validity. No significant correlations were found for players from Condition 2.

\subsubsection{Summary}

First, we investigated whether there were any relationships between performance indicators in the game and judgments on the perceived representational validity. Results showed that students who performed better in the game judged the game simulation model more critically. The influence of a poor performance in the game on validity judgments was not found.

Secondly, we looked at the relationships between representational validity and educational validity. Overall, results do not indicate that unidirectional and unequivocal relationships between perceived representational validity and educational validity exist in the case of the KM Quest game. However, a few significant correlations indicated that there are positive relationships between perceived representational validity and external educational validity. For players from Condition 2 there is a relationship between perceived internal representational validity and external educational validity, while for players from Condition 1 there is a relationship between perceived external representational validity and external educational validity. This result is not contradictory because players from Condition 1 judged the model consisting of knowledge process related variables as being a valid representation of reality less positively.

The absence of relationships between perceived representational validity and educational validity can be explained by noting that overall students assessed the game simulation model positively and that the model itself possesses representational validity to some extent. If students would experience an implausible or completely unrealistic model, then we could assume that appreciation of the model as being a valid representation of reality could have an influence on the playing behavior, and consequently, on the performance indicators and learning outcomes.

\subsection{Discussion}

In this experiment we investigated how the presence or absence of knowledge process related variables for players of the game influenced the model's educational and representational validity. By presenting or excluding these variables, we varied the model complexity for players as well as the model's fidelity, which affected the representational validity. These knowledge process variables are mostly unknown and are not used in reality. They formalize abstract and hard to observe phenomena that nonetheless influence the knowledge household of a company and, as a consequence, its business performance. Knowing these abstract phenomena, in our view, helps individuals to learn about relationships between the dynamics of knowledge processes in a company and a 
company's business outcomes. In this section the results and findings from the experimental study are discussed.

The results of game performance revealed that if knowledge process related variables are presented to the players of the game, they pay attention to these variables. Students having this information available achieved higher values on indicators of the level of knowledge in the company; this is possible almost only if a player has access to the knowledge process related variables and their changing values over game periods. At the same time, the presence of these unknown variables lead to more critical judgments about the model in terms of its acceptance as a valid representation of the real world. However, this critique does not influence the model's educational validity, since we did not find a negative correlation between the perceived external representational validity of the model and the model's external educational validity. To the contrary, a better performance in the game decreases the judgment of the model as a valid representation of a real world situation. A few indications of relationships between perceived internal representational validity and internal educational validity do not prove that the perceived internal representational validity has an influence on playing behavior and internal representational validity although, for one condition there were positive correlations between perceived internal representational validity and external educational validity.

\subsubsection{Educational validity of the model: hypotheses 1 and 2}

Internal educational validity in our study meant the ability of the student to discern the relationships of the modeled phenomenon and to address them in the intended manner. We have measured the internal educational validity of the model with three performance indicators in the game and assessed the conceptual knowledge of students after playing the game in the knowledge test. In general, there were no significant differences in performance between the two experimental conditions on two of the performance indicators, profit and number of interventions. This outcome could be explained by the fact that during the game players in both conditions followed a systematic decisionmaking normative model of knowledge management and, additionally, they had at their disposal a game feedback function where they could find information about the effects of events and the interventions that should be taken to counter or propagate these effects. From the log-files we found that players in both conditions frequently implemented interventions recommended in this feedback. It could influence players' behavior in the game, because in both conditions students applied a large amount of interventions that caused no differences in the outcomes of the number of interventions and profit indicators for the conditions. If students in Condition 1 would have had access to the supportive normative knowledge management model, which can help students to find knowledge bottlenecks and therefore to chose right interventions, then we could find more differences between the performances on these two indicators. We assume that if students in both conditions had stricter limitations in the game, such as the number of interventions allowed to implement per game period or a lower game budget, this would have forced players to think more deeply about the strategies used and the allocation of resources in the game. Nevertheless, we found that students who had the opportunity to access the values of the knowledge process related variables achieved higher values on 
the knowledge level indicator. This showed that students pay attention to these variables in the game and think about the relationships between knowledge processes and organizational outcomes because students in Condition 1 showed significantly better results on the knowledge test assessing conceptual knowledge. Thus, our hypothesis (H1) that the model including knowledge process related variables and their presentation to the players would provide a higher internal educational validity, is confirmed.

We defined external educational validity as the ability of students to develop strategic knowledge in the knowledge management domain and thus be able to solve problems in this domain. The results indicate that students who had access to the knowledge process variables in the model, showed better results on the knowledge test assessing strategic knowledge. This can be explained by the fact that monitoring the behavior of these knowledge process related variables or simply representing them, challenged students to think about relations between knowledge in the company and its organizational performance. Additionally, the detailed description of the knowledge level might have provided this deeper insight into the modeled phenomenon. The hypothesis $(\mathrm{H} 2)$ that the model including knowledge process variables and presentation of these variables to the players of the game provides a higher external educational validity is confirmed.

In general these findings support the idea that formalization and quantification of a new abstract concept can be helpful in the teaching of such concepts.

\subsubsection{Perceived representational validity: hypotheses 3 and 4}

The results show that, in general, the players of the game positively judged the model's representational validity. Players with the model in which we did not display the knowledge process related variables judged the model more positively on almost all items than players with the model in which knowledge process variables were presented. Although this finding is in line with our expectation that the presence of unknown variables lowers a model's external representational validity, our hypotheses $\mathrm{H} 3$ and $\mathrm{H} 4$ are rejected since players in Condition 1 gave lower scores in their judgment of the model on only three validity propositions assessing external representational validity and on only one validity proposition assessing internal representational validity. Nevertheless, these items represent important elements of representational validity of the model and the related findings jeopardize the representational validity of the model. Thus, variables that are unknown and unfamiliar in reality negatively influence to a certain extent the perceived internal and external representational validity of the model.

\subsubsection{Relationships between the educational validity of the model, its fidelity and perceived representational validity}

Our expectation that the model, which includes knowledge process variables thus possessing a lower level of physical and a higher level of functional fidelity, will lead to lower perceived representational validity is not met. Moreover, based on circumstantial evidence, we conclude that the presence of knowledge process variables positively influences the internal and external educational validities of the model and the judgment 
of the model as a valid representation of reality, does not negatively influence the internal and external educational validities of the model. This finding is not counter intuitive. First of all, in this study subjects judged the representational validity of the model positively overall, which can explain the absence of significant relationships between representational and educational validities. Secondly, the game simulation model possess to some extent representational validity as a result of the process of model development and adjustment during the KITS project which grounded it into the experience of seasoned knowledge management consultants.

From all possibilities for studying the model's effectiveness, we dealt in this experiment with only one internal factor - the model's fidelity. In the next experiment we will investigate if and how an external factor can influence the model's representational and educational validities. 


\section{Chapter 6}

\section{Mode of playing: investigating validity}

In the previous chapter we described an experiment in which we investigated how different levels of the model's fidelity or model characteristics presented to the learners influenced representational and educational validity of the model. This chapter is devoted to an experimental study in which we investigated the influence of a factor external to the model (i.e. mode of playing) on the model's representational and educational validity. Additionally, in this experimental study we changed the model presented to the players again: players in both conditions could not observe the business process related variables. Compared to the first study we decreased both functional and physical fidelity of the model. How does it affect educational and representational validity of the model? The chapter begins with the purpose of the study and its research questions, followed by the description of the experiment itself, in which the experimental design, procedure and results are presented. The chapter ends with explanation of the results and discussion. 


\subsection{Introduction}

\subsubsection{Purpose of the study}

In the previous chapter we described how a model's characteristic, its fidelity, influences the representational and educational validities of the model. In this chapter we address the validity of the model from a different perspective. We investigate how a factor external to the model, the mode of playing, influences the model's representational and educational validity. Does individual or collaborative learning influence the representational and educational validity of the model? Which mode of playing leads to a higher educational validity and a higher acceptability of the model as a valid representation of reality? Would it be effective for players to play the game individually or is collaboration and learning in teams a necessary condition for the effective acquisition of decision-making skills in the game?

Additionally, following the research in the previous chapter, in this study we included more research on the effect of the model's fidelity and the model's representation to the players on the educational and representational validity. We decreased the model's physical and functional fidelity: players could not observe behavior of the business process related variables. How would this affect the model's representational and educational validity? Does collaboration play a role in the acquisition of conceptual and strategic knowledge in an environment where the link between conceptual and strategic knowledge is not obvious?

This study intends to find an answer to these and related questions.

\subsubsection{Subject matter}

One of the six major dimensions along which computer business games are characterized is mode of playing: individual or in teams (Faria \& Schumacher, 1984). In the gaming and simulation field, research has been conducted on the differences between individuals and teams in terms of game performance, learning outcomes and decision-making behavior.

Some authors reported that group-play facilitates better leaning (Chanin \& Wolfe, 1993; Slavin, 1990). In an experimental study where the effects of four team sizes were studied in a complex business game, Wolfe and Chacko (1982) found that 3-member teams obtained the highest learning outcomes and highest performance results, while 2member teams experienced marginal learning effects and performance results. Individuals showed the smallest knowledge increase and poor performance. Fourmember teams were not significantly different from 3 member teams. The authors argued that "given the richness of the simulation's basic complexity, a game will produce different results due to team size effects"(p. 234) and "an optimal team size might exist for maximum student learning and that different team sizes may produce unique learning environments and results" (p. 232). They invite researchers to do rigorous experimenting with the games in order to produce clearer results in this respect.

Also educational science pays great attention to collaborative learning in games. Although the results of many studies are contradictory (Kocher \& Sutter, 2005), researchers believe that when students work together to solve problems, they freely 
verbalize their knowledge, understanding, problem-solving strategies and misconceptions (Shavelson, Webb, Stasz \& McArthur, 1988). "When the game is repeated, groups learn the dynamics of the game significantly faster [...] and increase their depths of reasoning"( Kocher \& Sutter, 2005, p. 219). From another point of view Kayes, Kayes and Kold (2005), in their research on experiential learning in teams, mentioned several factors that could negatively affect team performance and member satisfaction: overdependence on a dominant leader, diffusion of responsibility, social loafing and a tendency to make risky or more conservative decisions than individuals acting alone. In a business game study reported by Newgen, Stair and Kuehn (1980), individuals learned their tasks and made their decisions faster than three member teams. Nevertheless, we believe that collaboration brings additional value in acquiring implicit or decision-making skills, because collaboration with other students provokes activity, makes learning more realistic, and stimulates motivation (Veerman \& Veldhuis-Diermanse, 2001) "In collaborative learning settings, learners are "forced" to share perspectives, experiences, insights, and understandings. This can help learners to come up with new ideas, debug their ideas, and notice the complexities of concepts and skills" (Leemkuil et al., 2003). When students work collaboratively, the more knowledgeable partner provides explanations to the less knowledgeable partner (Teasley, 1995). Even when none of the students in a discussion group originally knows the correct answer, peer discussion enhances understanding and "increases the percentage of correct answers" (Smith et al, 2009). They claim that this results "from gains in understanding during discussion, or, simply from peer influence of knowledgeable students on their neighbors" (Smith et al, 2009 , p.122). Thus, we believe that collaborative learning is a promising approach in learning to solve problems in ill-defined domains, such as knowledge management, and for acquiring strategic knowledge, because students share their perspectives, ideas and experiences.

In the early evaluation studies of the KM Quest prototype (Christoph et al., 2003), which did not have all the game features that are available now, results showed that both individual and collaborative players were not able to improve or improved only minimally their knowledge base in the knowledge management domain. In the study reported by Purbojo (2005), in which players worked in teams collaboratively but were distributed locally in the KM Quest environment, no positive learning effects were found. Summarizing we can say that results of investigating collaborative learning in a game, are equivocal.

Contrary to this, no research has been done previously regarding how collaboration could affect one's judgment about representational validity, thus leaving open a new topic of research.

Hence, in this study we investigate (1) how individual or collaborative learning influences conceptual and strategic knowledge, as part of the educational validity in a context where the link between conceptual and strategic knowledge is fuzzy, and (2) how a different mode of playing affects judgments of players about the model's representational validity.

Since we are investigating the differences between possible influences of a collaborative or an individual mode of playing on the educational and representational validity of the model, the motivation to collaborate must be present. This premise influences the design and procedure of the current experiment in two ways. First, we 
allowed students to allocate themselves to a team. Initial group cohesion is important for the performance in a game environment, whereas the initial attitude of students has limited consequence for the performance (Wellington \& Faria, 1996). Secondly, we use the richest form of the communication (Daft \& Lengel, 1998) between players: face-toface, instead of allowing them to use chat facilities, because in collaborative settings chat communication puts some constrains on communication (Kollöffel, 2008). As Kollöffel (2008) states, face-to face communication "provides both verbal and non-verbal information (e.g., gesturing, nodding, pointing, facial expressions, and intonations of speech), [...] allows students to communicate faster and much more elaborate, which can be crucial in the case of interpretation and sense-making" (p. 61).

\subsubsection{The KM Quest simulation model and its support in the experiment}

In contrast to the previous experiment, the model represented to the players in this experiment possessed a lower level of both physical and functional fidelity. Players in both conditions could observe the knowledge process related variables (which decrease physical fidelity but increases functional fidelity of the model), but could not observe business process variables (which decrease physical and functional fidelity of the model). In this experiment we closed access to the second layer of the conceptual model, (see Fig. 2.3 , p. 24) and this minimized the relationship between conceptual and strategic knowledge (see Fig. 6.1), because players could not observe the propagation of knowledge management interventions influences on organizational outcomes via the business process variables. Access to the supportive KM model, which guides players in acquiring conceptual knowledge, was left available for players in both conditions. In fact, we created a fuzzy environment in which the acquisition of conceptual knowledge was explicit and the acquisition of strategic knowledge was even more implicit as compared to the original environment. This was done from an experimental point of view in order to see how collaborative and individual players would construct their conceptual and strategic knowledge in such a fuzzy environment and whether collaboration would play any role in acquiring strategic knowledge, even without an explicit link between conceptual and strategic knowledge (see Figure 6.1).

In order to acquire their conceptual knowledge players had to learn how KM interventions influence the knowledge process related variables. This they do by identifying variables having "low" values with guidance of supportive KM model and choosing appropriate KM interventions. In order to acquire their strategic knowledge players should recognize a KM problem and should be able to react to this problem. For this they have to understand how KM interventions influence organizational outcomes, thus they have to understand the propagation of KM intervention influences in order to solve KM problems or to improve organizational outcomes. In the model and environment for this experiment the propagation of $\mathrm{KM}$ intervention influences is not obvious, because players do not observe business process related variables. Players could see only three organizational outcome variables. By applying single or multiple interventions it was difficult to see how a particular intervention influenced these, due to the multiple and delayed effect of KM interventions and the decay behavior of knowledge processes. 
Closing access to the supportive KM model, therefore making the acquisition of conceptual knowledge impossible, would make the game even more difficult and maybe unplayable. Therefore the supportive KM model was left available.

Figure 6.1 Learning process in experimental KM Quest environment

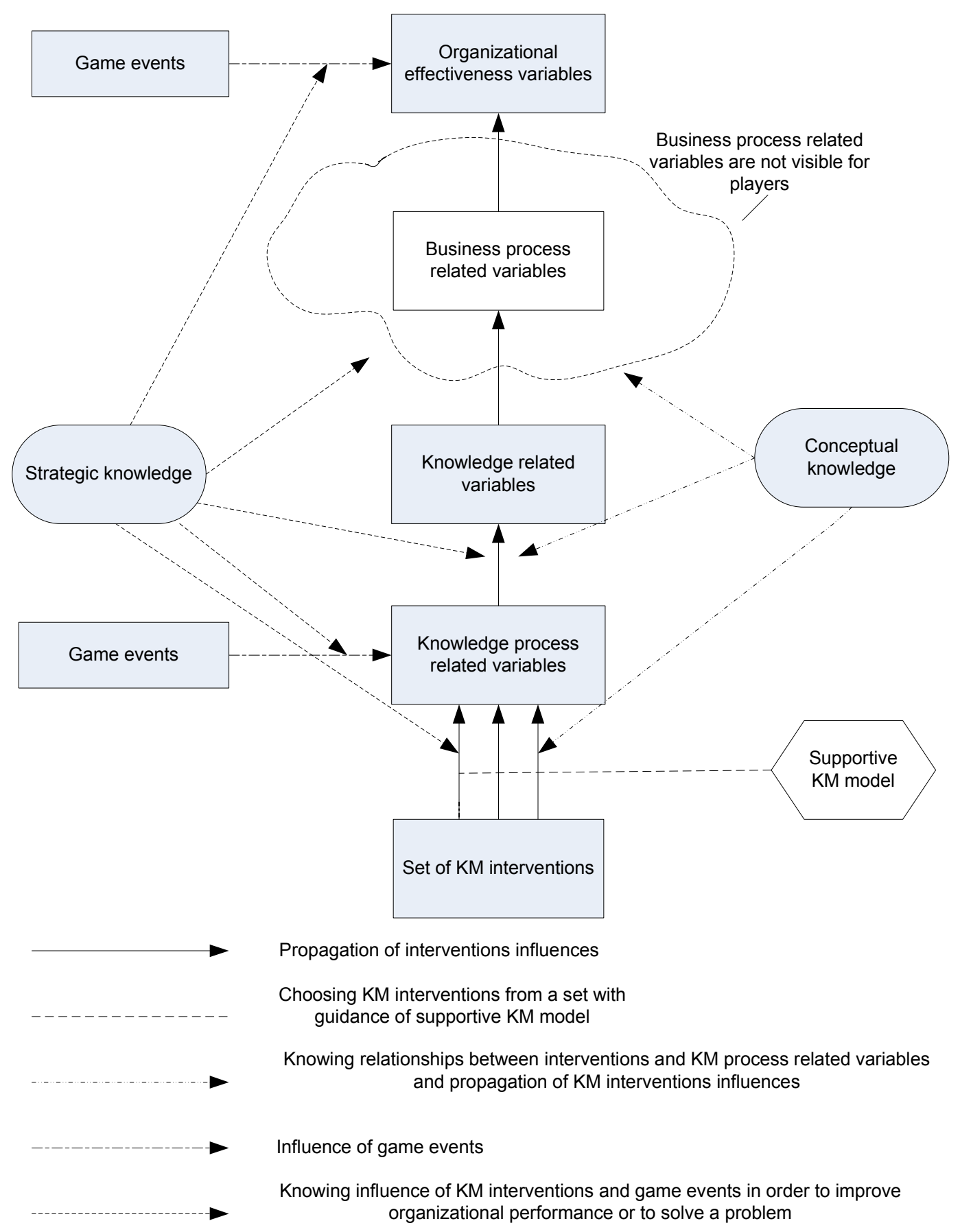




\subsubsection{Research questions and expected outcomes}

Although there is no obvious evidence or common agreement among educators on the benefits of collaborative performance versus individual performance, most of them strongly believe that collaboration helps in the acquisition of decision-making skills. In our study we investigate how the collaborative mode and the individual mode of playing differ from each other: which mode of playing lead to a higher educational validity and how the mode of playing influence the representational validity of the model.

We expect that the collaborative mode of playing leads to a higher internal and external educational validity of the model and the game than the individual mode of playing, because, as already was pointed out in the previous section, team players can exchange their ideas while playing and can support each other in understanding complex knowledge management problems. At the same time, the mode of playing influences players' judgments of the representational validity of the model because players in the collaborative condition could exchange their opinions about the 'unrealistic' model and if one of the teammates perceived the model as an invalid representation of reality, he or she could influence the opinion of the second player. Thus, the hypotheses were:

- H1: The collaborative mode of playing leads to a higher internal educational validity than the individual mode of playing

- H2: The collaborative mode of playing leads to a higher external educational validity than the individual mode of playing

- H3: The mode of playing influences perceived representational validity of the model.

Since in the set-up of the game for this experiment only three variables were familiar to the players from reality, we expected that the model's external representational validity would be judged lower than the internal representational validity, because in the game players had access to additional support about the knowledge and knowledge process related variables and they could see these variables as one of the game's elements.

\subsection{Method}

\subsubsection{Design and subjects}

The subjects in this study were 73 international and 23 Dutch students of Nijmegen School of Management (Radboud University Nijmegen), who participated in the course "Knowledge management" as a part of the Business Administration program. Participation in the game was one of the course assignments and was contributed to the final course grade. The subjects had different backgrounds since they followed a different curriculum for the first years of their study and they came from different countries. Twenty-four subjects had experience in business gaming, but none of them were familiar with knowledge management games and they did not study knowledge management or have any experience in knowledge management before participation in the course. Since subjects did not have experience in knowledge management, they were asked to allocate themselves into one of the two conditions: a collaborative face-to-face mode of playing in a team of two or an individual mode of playing. This was done for two reasons mentioned 
before: to avoid language and communication problems and to make sure that students that collaborated were motivated to do so.

The collaborative mode of playing was chosen by 52 international students and by 14 Dutch students, while the individual mode of playing was chosen by 21 international and by 9 Dutch students. This resulted in 30 individual players and 33 teams of two players.

Later on members of three teams asked to play the game individually. As a result, we had at the beginning of the experiment 36 individual players and 30 teams.

Being still aware of the sampling construction bias due to self allocation to one of the conditions, we used a pretest-posttest design (Krathwohl, 1988). This design was primarily chosen to see whether there were initial differences between subjects.

\subsubsection{Criterion measures}

\subsubsection{Educational validity of the model}

In this study we assessed which mode of playing provided a higher internal and external educational validity, as before, by means of a knowledge test and performance in the game.

The pre- and post knowledge tests used in this study aimed at measuring conceptual and strategic knowledge of players of the game in the knowledge management domain. The pre- knowledge test suits this study for assessing the differences in prior conceptual and strategic knowledge of the subjects regarding knowledge management. The postknowledge test suits this study for assessing which mode of playing leads to a higher internal and external educational validity. Although it can be argued that learning effects are part of the internal educational validity, this study did not aim to investigate learning per se and therefore the learning effects of playing the game are left out of investigation.

Conceptual knowledge in the game refers to the internal educational validity and is defined as the ability of players to discern relationships of the modeled phenomena, in other words players knowledge of the interventions' influences on the variables of the model.

Strategic knowledge in the game refers to the external educational validity and is defined as the ability of players to recognize a knowledge management problem and solve the problem by selecting appropriate knowledge management interventions. Strategic knowledge is based on conceptual knowledge.

The pre-knowledge test as well as the post-knowledge test was identical for both conditions.

For measuring conceptual knowledge we presented students with 5 knowledge management interventions (from those which were used in the game) and asked them to specify the influence of each of the interventions in terms of affected domains and type of influence - delayed or immediate (see Example 6.1). When evaluating the answers, we compared students' specifications with the interventions' specifications used in the model. These were taken as the standard because players of the game constructed their conceptual knowledge based on the general behavior of the game that was driven by the specifications in the model. 
Example 6.1 Pre-posttest item measuring conceptual knowledge

In this assignment, you have to specify the intervention' influence

Intervention: Conduct a training program that aims at improving employees' attitude and motivation.

1. Indicate which knowledge domains does the intervention influence

- Marketing

- Research and Development

- Production

- Other

2. Indicate which knowledge processes does the intervention influence

- Gaining of knowledge

- Development of knowledge

- Utilization of knowledge

- Transfer of knowledge

- Retention of knowledge

- Other

3. Indicate what kind of influence the intervention has

- Long-term influence

- Short-term influence

- Immediate influence

- Delayed influence

Each sub-question was assessed on three levels: 0 points - wrong answer or all items marked when only some are correct; 1 point - half of the correct answers marked; 2 points - correct answer. In total each intervention could bring 6 points and the entire test 30 points.

For measuring strategic knowledge we presented students with a description of 5 different events (for the pre- and post tests we used different events) that occurred in the same company as in the game (Coltec), but did not occur in their games. For each event, it contained: one multiple choice answer about the nature of the event, whether it was a problem or an opportunity event or neither, and two questions that required an openended answer (See Example 6.2).

The first question asked students to write down consequences of this event for the company and the second question asked students to select knowledge management interventions from a predefined list that would, in their judgment, prevent the effect of the event or would propagate its effect. In total this part of the test consisted of 15 items, 3 items for each event (one multiple choice item and 2 items requiring open-ended answer). As in the previous study decisions made by experts were taken as the standard against which students decisions were assessed. 
Example 6.2 Example of the pre- and posttest item measuring strategic knowledge

In this assignment, you as a knowledge manager of Coltec have to analyse five events that happened outside or inside of Coltec and take knowledge management initiatives.

\section{Description of Event}

Until now there has been a fairly stable preference of customers of coatings for a limited set of colours. Recent market research has shown that this preference will be less and less stable in the future leading to short term swings in demand, depending on the current colours en vogue.

1. Indicate what kind of event this is:
口 An internal problem/threat but not KM related
- An internal problem/threat that calls for KM actions
口 An external problem/threat but not KM related
口 An external problem/threat that calls for KM actions
口 An internal opportunity but not KM related
- An internal opportunity that calls for KM actions
a An external opportunity but not KM related
口 An external opportunity that calls for KM actions

2. Indicate what effect the event can have on knowledge processes, on specific types of knowledge and/or business indicators (Post-test question).

3. Based on the information you have so far, which (set of) knowledge management interventions would you propose to the management of Coltec and what are their goals?

If students' answers were matching the answer of at least one of the experts, his or her answer was counted as correct. For each event in the first item students could score 0.2 points for the right answer, 0.1 points if they choose one alternative when 2 were possible, and 0 points for an incorrect answer. On each of the open-ended questions students could score 0.4 points in case of a correct answer, 0.2 points in the case they did not mention all consequences of an event or did not select interventions to react to all consequences and 0 points for an incorrect answer. Overall each event could bring the student a maximum of one point and a total of five points for five events.

The second measure for internal educational validity used in this study was performance in the game. To compare performance in the game between subjects in the two conditions we used the same performance measures as in the previous study:

- Company Profit - an internal game indicator. This is the most commonly used indicator of company success. This indicator was accessible for players in both conditions. 
- K-value - an indicator assessing the level of knowledge in the company. Students in both conditions could observe knowledge process variables which contribute directly to the variables "Level of competence", the sum of which constitutes the $\mathrm{K}$-value indicator.

- Number of interventions chosen by a player $(\mathrm{N})$. This indicator reveals how effectively students selected their interventions based on the information in the system - events, interventions and values of observable indicators.

In accordance with our hypothesis $\mathrm{H} 1$ we expected that students who played collaboratively would achieve higher scores on the post-test items assessing conceptual knowledge and outperform individuals on performance indicators. We also expected that collaborative players would achieve higher scores on post-test items assessing strategic knowledge, which is in line with our hypothesis $\mathrm{H} 2$.

\subsubsection{Perceived representational validity}

We assessed perceived internal and external representational validity of the model with the same categories as in the previous study:

- Plausibility - the model and its components are regarded as adequate to learn about inferences in the game;

- Applicability- the model and its components could be used to perform knowledge management-like work in actual companies

- Credibility - the model and its components represent relevant and actual features of real product leadership companies.

Perceived internal representational validity was assessed with the plausibility category subjects had to evaluate model behavior and its components in the game as being plausible for learning relationships between knowledge, knowledge management interventions and organizational outcomes. Perceived external representational validity was assessed with the Applicability and Credibility categories - subjects had to evaluate the model and its components as being applicable and relevant to reality.

For measuring the perceived internal and external representational validity we used the same validity questionnaire (Appendix 7) as in the previous study. Again we measured all eleven modeling elements against the three validity categories on a six-point scale (from strongly disagree to strongly agree):

1. Conceptual model

2. Knowledge domains

3. Knowledge processes

4. The business process variables

5. The knowledge processes related variables

6. The knowledge management interventions

7. The events

8. The decay behavior of the model

9. The effects of interventions

10. The effects of events

11. The overall behavior of the model. 
In line with our hypothesis $\mathrm{H} 3$, we expected differences in judgments between students who worked individually and students who worked collaboratively. We expected that students, who work together would judge the model more critically, as they could see only three realistic variables and discuss 'unrealistic' variables and the model behavior while playing the game. At the same time, we expected that judgments on the internal representational validity would receive higher scores than judgments on the external representational validity, because the model that was observable by players consisted of only three indicators which are used in reality, making it less likely that the model would be seen as representing reality.

\subsubsection{The KM Quest environment in the experiment}

The game environment was the same in both conditions. From the game features a game feedback function was available to the players that provided information about the event which occurred in the previous quarter together with a list of possible interventions which should be taken to counter or propagate the effect(s) of the event. The advice and visualization features were excluded for both conditions, which minimized the number of game features in order to focus on the model's features. In both conditions the underlying behavior of the model was the same: applying the same intervention would lead to the same changes in the values of variables. In the experiment, students had to play 8 game quarters. We choose 8 game quarters because this duration of the game is sufficient to see the delayed effect of interventions and the overall behavior of the model. All games had the same initial values for all variables and the same sequence of events. This was done to guarantee that the initial conditions were the same for every individual or collaborative game. Otherwise different playing conditions and differences in the game environment could influence game performance and test results.

Performance in the game was recorded in the log files. From these log files we could see when a player was logged on to the game and the duration of the session and what he or she had done. For our criterion measures based on game performance, we relied on the following data from the log files: the values of the indicators in the game after each game period, the game budget and the selection of interventions by a player in each game period.

\subsubsection{Procedure}

At the beginning of the course Knowledge Management subjects were introduced to the KM Quest game during a regular lecture. In this introductory lecture they learned about the KM Quest concept, about the Coltec company and about knowledge processes that are used in the game. They received a written description of Coltec and the knowledge processes. After the introduction but during the same class lecture, subjects individually completed a paper-based pre-test and filled in a profile questionnaire. After a week, the computer-session was organized for individual players and one week later for players who played collaboratively. Teams played by sharing one computer. In both conditions players played the first 4 periods of the game in class with an instructor present. After this, they were asked to finish playing the last four periods of the game during the next 3 weeks in their spare time. Each game was evaluated against the game results, the 
achieved values of the performance indicators, which motivated the collaborative players to play the remaining game periods together, because they shared the mark for their performance. One week later, in a classroom session, participants individually completed the knowledge post-test and filled in the validity questionnaire. At the same session the game results of each team were presented to the players.

One individual player did not finish playing the game and was not allowed to participate in the post-test. One player from the collaborative condition did not appear at the post-test session. Two players from individual condition did not fill in the validity questionnaire; therefore their results were taken only into analysis of the game results and the test-scores.

\subsection{Results}

The log files revealed that three players who played individually overspent the game budget because they applied a large number of interventions. Their game performance data, test data and judgments on representational validity were excluded from the analysis, because these players did not play within the rules for all players.

At the end, we compared the game performance of 30 collaborative teams and 32 individuals. For the pre-and post-test data analysis we analyzed data from 59 players who played collaboratively and 32 individual players. For the perceived representational validity analysis we had 59 validity questionnaires from players who played collaboratively and 30 from players who played individually.

Prior to answering the research questions, we tested whether there were initial differences between two conditions concerning conceptual and strategic knowledge by means of pre-knowledge test. The reliability, Cronbach's $\alpha$, for the pre-test yielded .739 for the part assessing conceptual knowledge and .763 for the part assessing strategic knowledge, which is satisfactory. The results of a t-test for independent samples indicated that there was no significant difference between subjects in the two conditions concerning conceptual $(\mathrm{T}(89)=.295, \mathrm{p}>.05)$ and strategic knowledge $(\mathrm{T}(89)=1.885, \mathrm{p}>.05)$ before the experiment. Thus, self-allocation in teams did not lead to differences in knowledge management knowledge, which makes the two conditions similar in this respect.

\subsubsection{Educational validity of the model}

Internal educational validity: performance in the game

Performance in the game was assessed, as in the previous study, with three performance measures: Profit - cumulative profit of the company at the end of the game, K-indicator sum of the end values of the level of competence variables for the three knowledge domains, and $\mathrm{N}$ - the number of interventions taken during the game.

A good performance in the game meant achieving high values of the Profit and Kindicator while applying fewer interventions when compared with the standard: 32-40 interventions for 8 game periods. Table 6.1 shows the mean values and standard deviations of the three performance measures for both conditions. 
Table 6.1 Mean values and standard deviations of the three performance measures for the two conditions

\begin{tabular}{|c|c|c|c|c|}
\hline Measure & Condition & $\mathbf{N}$ & Mean & Std. Deviation \\
\hline Profit & $\begin{array}{ll}\begin{array}{l}\text { Condition } \\
\text { (collab.) }\end{array} & \\
\text { Condition } & 2 \\
\text { (ind.) } & \end{array}$ & $\begin{array}{l}30 \\
32\end{array}$ & $\begin{array}{l}79828157.10 \\
79998804.12\end{array}$ & $\begin{array}{l}7902728.361 \\
10955241.308\end{array}$ \\
\hline K & $\begin{array}{ll}\begin{array}{l}\text { Condition } \\
\text { (collab.) }\end{array} \\
\text { Condition } \\
\text { (ind.) }\end{array}$ & $\begin{array}{l}30 \\
32\end{array}$ & $\begin{array}{l}26.2007 \\
24.1341\end{array}$ & $\begin{array}{l}2.54446 \\
4.58906\end{array}$ \\
\hline$N$ & $\begin{array}{ll}\begin{array}{l}\text { Condition } \\
\text { (collab.) }\end{array} \\
\text { Condition } & 2 \\
\text { (ind.) } & \\
\end{array}$ & $\begin{array}{l}30 \\
32\end{array}$ & $\begin{array}{l}42.13 \\
37.72\end{array}$ & $\begin{array}{l}6.559 \\
11.280\end{array}$ \\
\hline
\end{tabular}

Although the standard deviations of the two conditions are quite different in their values, Levene's test showed that the variances of two conditions do not differ significantly. A t-test for independent samples showed that there are no significant differences in game performance between players in the two conditions on the Profit and $\mathrm{N}$ indicators ( $\mathrm{p}>0.05)$. Nonetheless, individual players (Condition 2 ) achieved almost the same values on the Profit indicator by implementing fewer interventions than the team players. Teams in Condition 1 achieved a lower value on the Profit indicator because they implemented more interventions than individual players in Condition 2 and the costs for implementing interventions are subtracted from the Profit. At the same time, teams outperformed individuals on the $\mathrm{K}$ - indicator. The mean values of the $\mathrm{K}$-indicator for collaborative players were significantly higher than the mean values of the $\mathrm{K}$ - indicator for individual players $(\mathrm{t}(60)=2.173, \mathrm{p}<.05)$. From these results we can conclude that $\mathbf{H 1}$ is only partially supported.

Internal and external educational validity: knowledge test

Again, as in the previous study, we measured conceptual and strategic knowledge with a post knowledge test. The reliability, Cronbach's $\alpha$, for the post test yielded .685 for the part assessing conceptual knowledge and .715 for the part assessing strategic knowledge, which is satisfactory. An independent samples t-test was used for identifying differences on the post- test scores between subjects in the two conditions.

Table 6.2 gives an overview of the mean post-test scores on the knowledge test assessing conceptual and strategic knowledge.

In terms of the post-test scores players who played collaboratively (Condition 1) showed significantly higher scores on the items assessing strategic knowledge than individual players $(\mathrm{Z}=2.244, \mathrm{p}<.05)$, no differences were found on items assessing conceptual knowledge. 
Table 6.2 Means and standard deviations (SD) of the post knowledge test scores for both conditions

\begin{tabular}{|c|c|c|c|}
\hline $\begin{array}{l}\text { Type of } \\
\text { knowledge }\end{array}$ & Condition & $\mathbf{N}$ & Post-test mean (SD) \\
\hline \multirow{2}{*}{$\begin{array}{l}\text { Conceptual } \\
\text { knowledge }\end{array}$} & $\begin{array}{ll}\text { Condition } & 1 \\
\text { (collab.) } & \end{array}$ & 59 & 20.78 (3.58) \\
\hline & $\begin{array}{l}\text { Condition } 2 \\
\text { (ind.) }\end{array}$ & 32 & $19.59(3.04)$ \\
\hline \multirow{2}{*}{$\begin{array}{l}\text { Strategic } \\
\text { knowledge }\end{array}$} & $\begin{array}{ll}\text { Condition } & 1 \\
\text { (collab.) } & \\
\end{array}$ & 59 & $4.18(.74)$ \\
\hline & $\begin{array}{l}\text { Condition } 2 \\
\text { (ind.) }\end{array}$ & 32 & $3.77(.90)$ \\
\hline
\end{tabular}

Our hypothesis $\mathrm{H} 2$, that the collaborative mode of playing in the given model would provide a higher external educational validity than the individual mode of playing was supported. As for internal educational validity, we did not find results which showed that the collaborative mode of playing provided a higher internal educational validity in comparison with the individual mode of playing, although teams achieved a higher value on the $\mathrm{K}$ - indicator. Our $\mathrm{H} 1$ is rejected.

Since the results for internal educational validity are ambiguous, we checked for a difference between the pre- and post knowledge test items assessing conceptual knowledge for subjects in both conditions in order to find out if subjects increased their conceptual knowledge. Table 6.3 gives an overview of the mean pre- and post-test scores on the knowledge test items assessing conceptual knowledge.

Table 6.3 Means and standard deviations (SD) of the pre-and post knowledge test scores for both conditions

\begin{tabular}{|l|l|l|l|l|}
\hline $\begin{array}{l}\text { Type of } \\
\text { knowledge }\end{array}$ & Condition & $\mathbf{N}$ & Pre-test mean (SD) & Post-test mean (SD) \\
\hline $\begin{array}{l}\text { Conceptual } \\
\text { knowledge }\end{array}$ & Condition 1 & 59 & $19.03(4.59)$ & $20.78(3.58)$ \\
\cline { 2 - 6 } & Condition 2 & 32 & $18.75(3.96)$ & $19.59(3.04)$ \\
\hline
\end{tabular}

The paired samples t-test indicated that the post-test scores on the conceptual knowledge test were significantly higher than the pre-test scores for players who played collaboratively $(\mathrm{t}(58)=-3.28, \mathrm{p}<.01)$. Players, who played individually, did not improve their conceptual knowledge significantly. This result supported our H1, that the collaborative mode of playing provides a higher internal educational validity.

\subsubsection{Perceived representational validity of the model}

The perceived representational validity of the model was assessed for players in both conditions using the validity questionnaire. The reliability of the entire validity questionnaire is equivalent to Cronbach's $\alpha=.916$, which is good. The reliability of the part assessing plausibility items yielded .739, the applicability part .812 and the credibility part .827 , which are also good. 
To find differences in judgments about perceived representational validity between the two conditions we used a t-test for independent samples. The mean judgment scores and standard deviations for both conditions are displayed in Table 6.3.

Table 6.3 Mean judgment scores and standard deviations (SD) on the representational validity items for both conditions

\begin{tabular}{|c|c|c|c|c|c|}
\hline Model components & Condition & $\mathrm{N}$ & Plausibility & Applicability & Credibility \\
\hline \multirow[b]{2}{*}{ Conceptual model } & Condition 1 & 59 & $4.66(.757)$ & $4.41(.931)$ & $3.95(.936)$ \\
\hline & Condition 2 & 30 & $4.67(.844)$ & $4.47(.730)$ & $3.70(1.05)$ \\
\hline \multirow[b]{2}{*}{ Knowledge domains } & Condition 1 & 59 & $4.34(.843)$ & $4.03(.946)$ & $4.15(1.03)$ \\
\hline & Condition 2 & 30 & $4.40(.894)$ & $4.27(.785)$ & $4.10(.885)$ \\
\hline \multirow[b]{2}{*}{ Knowledge processes } & Condition 1 & 59 & $4.39(.766)$ & $4.31(.876)$ & $4.34(.976)$ \\
\hline & Condition 2 & 30 & $4.53(.937)$ & $4.40(.770)$ & $4.20(714)$ \\
\hline \multirow{2}{*}{$\begin{array}{l}\text { The business process } \\
\text { variables }\end{array}$} & Condition 1 & 59 & 4.321 & $4.15(1$ & 4.10 \\
\hline & Condi & 30 & 13) & 4.30 & 4.20 \\
\hline \multirow{2}{*}{$\begin{array}{l}\text { The knowledge process } \\
\text { related variables }\end{array}$} & Condition 1 & 59 & & 5) & 3.88 \\
\hline & Condition 2 & 30 & $4.40(1.00)$ & $4.00($. & $3.83(.950)$ \\
\hline \multirow[b]{2}{*}{ The interventions } & Condition 1 & 59 & $4.14(1.36)$ & $4.02(1.28)$ & $4.08(1.13)$ \\
\hline & Condition 2 & 30 & $4.33(1.02)$ & $4.33(.922)$ & $4.17(1.02)$ \\
\hline \multirow[b]{2}{*}{ The events } & Condition 1 & 59 & $4.19(1.11)$ & $4.47(1.04)$ & $4.14(.937)$ \\
\hline & Condition 2 & 30 & $4.30(.877)$ & $4.53(.900)$ & $4.50(.974)$ \\
\hline \multirow{2}{*}{$\begin{array}{l}\text { The decay behavior of the } \\
\text { model }\end{array}$} & Condition 1 & 59 & $4.10(.855)$ & $4.00(.851)$ & $3.97(.999)$ \\
\hline & Condition 2 & 30 & $4.37(.718)$ & $4.30(.915)$ & $4.20(.887)$ \\
\hline \multirow[b]{2}{*}{ The influence of interventions } & Condition 1 & 59 & $4.05(1.10)$ & $3.68(1.25)$ & $3.64(1.03)$ \\
\hline & Condition 2 & 30 & $4.07(1.02)$ & $4.13(1.01)$ & $3.90(1.09)$ \\
\hline \multirow[b]{2}{*}{ The influence of events } & Condition 1 & 59 & $4.14(1.17)$ & $3.97(1.10)$ & $3.75(1.09)$ \\
\hline & Condition 2 & 30 & $4.23(1.01)$ & $4.27(.785)$ & $3.97(.999)$ \\
\hline \multirow[b]{2}{*}{ The behavior of the model } & Condition 1 & 59 & $4.17 .968)$ & $3.98(.919)$ & $3.88(.948)$ \\
\hline & Condition 2 & 30 & $4.27(.785)$ & $4.20(.847)$ & $3.97(.928)$ \\
\hline \multirow[b]{2}{*}{ Aggregation } & Condition 1 & 59 & $46.85(5.94)$ & $44.81(6.62)$ & $43.88(6.28)$ \\
\hline & Condition 2 & 30 & $47.60(5.14)$ & $47.20(5.67)$ & $44.73(7.24)$ \\
\hline
\end{tabular}

There were no significant differences found between judgments of subjects in Condition 1 and Condition 2 on perceived representational validity. As we did not find significant differences between judgments of players in the two conditions our H3 is rejected.

In general all plausibility validity propositions were judged by subjects in both conditions to be above a ranking of 4 (from slightly agree to agree), while subjects in both conditions assessed the following credibility validity propositions to be below a ranking of 4 (from slightly disagree to slightly agree, bold Italic in the Table 6.3):

$\circ$ The credibility of the conceptual model;

- The credibility of the knowledge process related variables;

- The credibility of the influences of interventions;

- The credibility of the influences of the events;

- The credibility of the model behavior. 
Given these overall scores, it can be said that the players judged the representational validity of the model fairly positively.

Although we did not find significant differences for the applicability validity propositions, subjects in Condition 1 judged all applicability propositions lower than subjects in Condition 2 and the following applicability propositions were assessed below 4 by subjects in Condition 1: applicability of the knowledge process related variables, applicability of the influences of interventions and events, and applicability of the model behavior.

This is in line with our idea that subjects would be more critical about external representational validity of the model because the model consists of only three variables used in reality. As for the hypotheses, we cannot conclude that mode of playing influences judgments about perceived representational validity. From another point of view, if there was an additional stimulus for the collaborative mode of playing, such as to discuss the model and the components of the model with your teammate, the results could be different, because collaboration facilitates sharing of opinions and teammates could affect each other's judgment.

\subsection{Discussion}

In this experiment we investigated how the mode of playing influences the model's educational and representational validity. In this study the model presented to the players of the game possessed a low level of physical and functional fidelity compared with the models used in the previous experiment, because players could not observe the business process related variables, which makes acquisition of conceptual and consequently strategic knowledge very difficult. Therefore players in both conditions were supported in their acquisition of conceptual knowledge by the supportive KM model.

Results of the game performance indicators for internal educational validity reveal that collaborative players achieved significantly higher values on the $\mathrm{K}$ indicator than individual players. No differences were found between collaborative and individual games on the Profit and $\mathrm{N}$ indicators. Achievement of a higher value on the $\mathrm{K}$ indicator signifies that collaborative players worked more precisely than individuals when choosing KM interventions with the guidance of the supportive KM model. Although we did not find differences between collaborative and individual players on the test scores assessing conceptual knowledge, the paired samples t-test revealed that collaborative players improved their conceptual knowledge after playing the game while individuals did not. These results allow us to conclude that the collaborative mode of playing provides a higher internal educational validity in the given model and game conditions. As for external educational validity, the collaborative players showed significantly higher scores on the knowledge test items assessing strategic knowledge than the individual players. Collaboration positively influences the acquisition of decision-making skills and provides a higher external educational validity.

We did not find significant evidence that collaboration influences the judgments of players on the internal and external representational validity of the model. If we would provide players with additional assignments to evaluate the behavior of the model or to check propagation of the interventions' influences at the moment they were working with the model, then it is likely that the perceived representational validity would be judged 
differently and that the differences between judgments of collaborative and individual players could occur, because collaboration assumes the sharing of meaning. Since the model included only three variables known in reality, players overall judged the perceived external representational validity lower than the perceived internal representational validity.

Compared to the previous study, we found that not only internal factors such as the model's level of fidelity but also external factors, from the model's point of view, influence the model's educational validity. On the other hand, one external factor did not have any influence on the perceived representational validity, which is to be expected, because this factor (collaborative or individual playing) is not relevant for the model's properties and features as they are given in the game. Finally it should be noted that overall judgments about the representational validity of the model were above average, indicating that players saw the model as being not too different from real organizations and their behavior concerning knowledge and knowledge management.

Since in this experiment we had a different model representation for the players than in the previous experiment, it is interesting to investigate how differences in the model's representation across both experiments affect the model's educational and perceived representational validity. This will be taken up in chapter 8 . 

Chapter 7

\section{Exploring representational validity}

While in previous chapters we investigated the perceived representational validity and educational validity of the model in the series of experiments, in this chapter we deepen our research on the representational validity of the model using a different target group experts in the knowledge management domain. 


\subsection{Purpose of the investigation}

In the previous chapters we investigated the educational validity of the model and the perceived representational validity with players of the game. The research on the model's validity would be incomplete if we do not address the other end of the validation continuum - representational validity. As we indicated in Chapter 4, the model needs to possess representational validity to some extent in order to be educationally valid. Initial research on the representational validity was completed within the frame of the KITS project during the model development and evaluation phases. Several authors (Irvine, Levary \& McCoy, 1998; Peters, Vissers \& Heijne, 1998) stated that during the design and validation process of any simulation model judgmental biases can enter because most validation procedures for simulation models are subjective-human judgments affect their outcomes. Therefore we conducted research on the representational validity of the model with subject matter experts in the knowledge management domain that were not involved in the KITS project in order to see to which extent the model is representatively valid according to their judgement.

\subsection{Design of the validation study}

\subsubsection{Research techniques}

As we indicated in chapter 4, for a model which does not have a 'real' reference object and which represents an abstract reality, only informal validation techniques which rely heavily on human reasoning and subjectivity (Balci, 1998), can be used in the investigation of the model's validity.

From the simulation research field we adopt a combination of two techniques Face validation and Walkthroughs. Face validation assumes that people knowledgeable about the system under study, based on their estimates and intuition, subjectively compare model and system behaviours and judge whether the model and its results are reasonable. The only difference for in the case of the KM Quest game simulation model is that experts have to compare model behaviour to an abstract reference system. Thus, the experts, while playing the game, have to assess the model behaviour against what they know about the subject. As Moya, McKenzie and Nguyen (2008) stated, most validation efforts account for some type of results validation, that is actual outcomes of the simulation; less attention is paid to conceptual model development. Therefore we applied the structured walk-through technique to address the validation of the conceptual model. In the structured walk-through method the object under study is a document which clarifies to the experts what simplifying assumptions were made when building the conceptual model. Experts were asked to give their opinion on these assumptions.

\subsubsection{Research instruments}

As we saw from Figure 4.1 (Chapter 4), the cycle of simulation model development begins with questions involving external validity - how correctly phenomena are modelled. In this sense it is related to content and construct validity. Next, the validation 
questions concern the game logic and structure, whether the model is internally consistent, and what refers to the internal validity.

From a practical point of view, in this study we could not address all modelling assumptions and decisions that were made in the design process of the game simulation model, as this would have overloaded the experts. Therefore, we choose a pragmatic approach and selected the most crucial modelling decisions concerning the correctness of the modelled phenomena reflecting the model's external representational validity to study in this investigation. These modelling assumptions and statements were incorporated into a modelling questionnaire, the assumption document, which consists of 5 items. These items represent the following crucial aspects of the model: the selection of the knowledge domains (Statement 1) and knowledge processes (Statement 3) for the description of the knowledge household of a product leadership company; the importance of knowledge decay for each knowledge domain (Statement 2); the contribution of knowledge to the business unit outcomes (Statement 4) and the relationships between knowledge processes and organizational outcomes (Statement 5). The complete modelling questionnaire can be found in Appendix 8.

Since participants of the game have to learn how KM interventions influence knowledge in the company, and that interventions as well as events are parts of the game's logic and structure, we choose to approach the model's internal representational validity by asking experts about specifications of KM interventions. The specification of $10 \mathrm{KM}$ interventions constitutes the second part of the modelling questionnaire (Appendix 8).

However, these statements and specifications do not provide a feeling of how everything works together. Therefore we asked experts to play the game as well and to fill in the validity questionnaire (Appendix 7), which we used for the experimental studies. The questionnaire assess the model and model elements in terms of plausibility (which reflect internal representational validity), applicability and credibility categories (which both reflect external representational validity).

These instruments cover both internal and external representational validity of the model.

\subsubsection{Subjects}

After a call for participation, 10 subject matter experts agreed to participate. Subject matter experts were academicians and practitioners in the area of intellectual capital, information management and knowledge management. Nine experts were from the Netherlands and one expert was from Russia. None of the experts were involved in the development of the KM Quest game. Two experts use the KM Quest game in their teaching.

\subsubsection{Procedure}

The experts were asked to work individually or with the assistance of the model developer to perform the following validation tasks:

- To complete the first part of the modelling questionnaire, which consisted of 5 modelling assumptions and statements about the model. Experts were asked to 
give their opinion about each statement on a six-point rating scale (from strongly disagree to strongly agree) and in the case of an unfavourable score they were asked to write down their point of view of the particular assumption.

- To complete the second part of the modelling questionnaire, which consisted of a description of 10 interventions with specifications of the influence on the knowledge domains and processes in terms of presence of the influence, direction of influence (positive or negative) and timing of the influence (immediate or delayed). The interventions were randomly selected from the pool of 56 interventions available to players in the game. If experts disagreed with our specifications, they were asked to specify for each intervention the nature of influences they thought would be better.

- To play the game for 8 game periods. The games were stored in log files.

- To fill in the validity questionnaire.

Assistance of the model developer was sometimes needed to reduce the work load of experts becoming familiar with the game. The total work load for an expert was between 3, 5 hours (with assistance of the model developer) and 8 hours. Six experts worked individually and 4 experts worked with the assistance of the model developer. One expert, who was working individually, completed only the first task in the whole procedure, so his data were not included in the analysis. The validity questionnaire could be filled in only after playing the game. For the remaining tasks all experts were free to choose their own work sequence. Experts could like or dislike the game in case of playing it first, but we did not consider this subjective factor as having a substantial influence on the judgements. In case of liking the game, experts were still asked to provide their opinion on the modelling assumptions and in the case of disliking the game, these opinions would be even more explicit and helpful.

\subsection{Results}

Results are presented based on the three validation tasks: judging the assumptions behind the conceptual model, judging the specifications of the interventions, and judging the validity propositions in the validity questionnaire.

\subsubsection{Conceptual model}

As the complete modelling questionnaire can be found in Appendix 8, Example 7.1 briefly introduces these modelling assumptions and statements without an explanation of why they were made. 
Example 7.1 Modelling assumptions and statements

Modelling assumption and statement 1: The Marketing, R\&D and Production knowledge domains are sufficient to represent the knowledge household of a company and need to be modeled to represent a fictitious product leadership company in the game context.

Modeling assumption and statement 2: Speaking about identified knowledge domains for a product leadership company, we assume that the most crucial area is R\&D, since the company competes on the development of innovative products. Marketing and sales knowledge has an average priority since the company has to market those products skillfully and know market requirements. The production knowledge domain has the lowest priority.

Modeling assumption and statement 3: Knowledge processes such as knowledge gaining, knowledge development, knowledge utilization, knowledge transfer and knowledge retention are needed to represent knowledge processes in a company and are sufficient to model a fictitious product leadership company in the game context.

Modeling assumption and statement 4: The business outcomes of any unit of a company depend on the level of knowledge (competence) and the efficiency of knowledge usage in this unit, if there are no additional influences from outside the unit. This statement is valid to be modeled in the game context.

Modeling assumption and statement 5: (See Figure 2.4)

a) Level of knowledge (level of competence) in a business unit depends on the efficiency of knowledge gaining, knowledge retention and knowledge development. Depends means how well and effective those processes are organized in the company: $b$ ) Efficiency of knowledge utilization depends on the organizational infrastructure of the company and the level of employee satisfaction; c) Changes outside or inside the company influence the knowledge infrastructure, knowledge areas and knowledge processes. These assumptions are valid to be modeled in the game context to provide an understanding of how knowledge and knowledge processes contribute to the unit or firm's outcomes.

Table 7.1 shows descriptive statistics for each modelling assumption and statement.

Table 7.1 Mean value, ranges, and standard deviations of five modelling assumptions and statements

\begin{tabular}{|l|c|c|c|}
\hline $\begin{array}{c}\text { Modelling assumption and } \\
\text { statement }\end{array}$ & N & $\begin{array}{c}\text { Mean (Min. - } \\
\text { max.) }\end{array}$ & Std. Deviation \\
\hline Modelling assumption and statement 1 & 9 & $4.44(3-5)$ & .882 \\
\hline Modelling assumption and statement 2 & 9 & $4.78(4-6)$ & .667 \\
\hline Modelling assumption and statement 3 & 9 & $4.78(3-6)$ & 1.202 \\
\hline Modelling assumption and statement 4 & 9 & $4.56(2-6)$ & 1.333 \\
\hline Modelling assumption and statement 5 & 8 & $4.25(3-6)$ & 1.035 \\
\hline
\end{tabular}

The mean ranks of all modelling assumptions and statements behind these assumptions ranged from 4 (slightly agree) to 5 (agree), which is sufficient to accept the modelling 
assumptions as valid. Three of the five modelling assumptions have standard deviations larger then one, so we looked at the distribution of the judgments. Judgments on the modelling assumptions 1 and 3 have bimodal distributions. This bimodality is not located at the extreme ends of the scale. Two experts responded to these statements with the answer 'slightly disagree' while the next possible score - 'slightly agree' was the response given by only one expert, indicating overall a positive skew. In general, the distribution of judgments is skewed to the positive end of the scale. This positive skew supports the overall conclusion that the assumptions are seen as making sense.

Since we investigate the extent to which the model possesses representational validity, we not only use a descriptive relative scale but also calculate the validity percentage for each modelling assumption separately.

In Table 7.2 the frequencies of experts' scores and the validity percentage for each modelling assumption and statement are presented. The modelling assumption is considered to be valid if it is ranked by experts from slightly agree to strongly agree and not valid if it is ranked by experts from slightly disagree to strongly disagree.

Table 7.2 Frequencies of experts' scores and validity percentage for each modelling assumption

\begin{tabular}{|l|l|l|l|l|l|l|l|l|l|}
\hline Statement & $\mathbf{N}$ & $\begin{array}{l}\text { Strongly } \\
\text { agree }\end{array}$ & Agree & $\begin{array}{l}\text { Slightly } \\
\text { agree }\end{array}$ & $\begin{array}{l}\text { Valid } \\
\%\end{array}$ & $\begin{array}{l}\text { Slightly } \\
\text { disagree }\end{array}$ & Disagree & $\begin{array}{l}\text { Strongly } \\
\text { disagree }\end{array}$ & $\begin{array}{l}\text { Not } \\
\text { Valid } \\
\%\end{array}$ \\
\hline Statement 1 & 9 & & 6 & 1 & 77.8 & 2 & & & 22.2 \\
\hline Statement 2 & 9 & 1 & 5 & 3 & 100 & & & & 0 \\
\hline Statement 3 & 9 & 3 & 3 & 1 & 77.8 & 2 & & & 22.2 \\
\hline Statement 4 & 9 & 2 & 4 & 1 & 77.8 & 1 & 1 & & 22.2 \\
\hline Statement 5 & 8 & 1 & 2 & 3 & 75.0 & 2 & & & 25.0 \\
\hline
\end{tabular}

All the modelling assumptions have a validity percentage of 75 percent or above, which is acceptable. Therefore, we consider the separate modelling assumptions as being valid. The average validity percentage for all modelling assumptions and decisions is above 81 percent, which is satisfactory and for that reason we consider the model as possessing external representational validity.

Table 7.3 presents several comments made by experts on each modelling assumption and statement. This table shows the types of improvements that could be made in the model. 
Table 7.3 Experts' comments on the modelling assumptions and statements

\begin{tabular}{|c|c|}
\hline $\begin{array}{l}\text { Modelling } \\
\text { assumptions } \\
\text { and statements }\end{array}$ & Experts comments \\
\hline $\begin{array}{l}\text { Modelling } \\
\text { assumption and } \\
\text { statement } 1\end{array}$ & $\begin{array}{l}\text { - Organisational domain could be added } \\
\text { - For a production company, procurement and service department } \\
\text { could be an important knowledge domains as well }\end{array}$ \\
\hline $\begin{array}{l}\text { Modelling } \\
\text { assumption and } \\
\text { statement } 2\end{array}$ & - Priority: $\mathrm{R} \& \mathrm{D}$, then production, then marketing \\
\hline $\begin{array}{l}\text { Modelling } \\
\text { assumption and } \\
\text { statement } 3\end{array}$ & $\begin{array}{l}\text { - I miss knowledge creation as an explicit process. I guess that } \\
\text { knowledge development includes it, but in that case it includes too } \\
\text { many different processes: knowledge creation, individual learning } \\
\text { and organizational learning. In my opinion the later has different } \\
\text { logic and problems, so I would rather include it as a part of } \\
\text { knowledge transfer. Also transfer of knowledge within a domain } \\
\text { could be important as an explicit process (e.g. knowledge } \\
\text { exchange between R\&D units in different locations (or even } \\
\text { within R \&D units if there are large enough (let's say more than } \\
100 \text { people) } \\
\text { - I miss integration/combination between the domains } \\
\text { The process that is missing is the process of reflection. The } \\
\text { process in which people deliberately learn from the things } \\
\text { they do }\end{array}$ \\
\hline $\begin{array}{l}\text { Modelling } \\
\text { assumption and } \\
\text { statement } 4\end{array}$ & $\begin{array}{l}\text { - These items to be confusion between knowledge domain and } \\
\text { (business) unit. The effectiveness of a unit depends first and } \\
\text { foremost on the organizational design, the effectiveness of a } \\
\text { domain on competence, usage and organizational embedding } \\
\text { - Knowledge is not only internal factor that affects the } \\
\text { business outcomes. There is also structural capital (e.g. ways } \\
\text { of working) and relational capital (e.g. networks) } \\
\text { - Also the ability to learn is important, e.g. single loop } \\
\text { learning where people learn from their own mistakes. This } \\
\text { aspect is not incorporated yet }\end{array}$ \\
\hline $\begin{array}{l}\text { Modelling } \\
\text { assumption and } \\
\text { statement } 5\end{array}$ & $\begin{array}{l}\text { - Many feedback loops could be added: e.g. level of } \\
\text { competence influences knowledge gaining and development } \\
\text { - The model is simplification as any model is } \\
\text { - There should be influence from level of competence to } \\
\text { knowledge utilisation } \\
\text { - As I do not completely understand the model, it is difficult } \\
\text { for me to agree/disagree. I need more information. }\end{array}$ \\
\hline
\end{tabular}

Experts commented most frequently about knowledge processes that were not incorporated into the model, processes such as knowledge creation, reflection and integration and about the relationships between level of competence and knowledge utilisation. The model would become too complex if we would incorporate all existing 
knowledge processes into the model. As the model intends to represent processes at a fairly high level of abstraction, processes at the level of an individual, for example individual learning and reflection, are left out as they would transform the model into a type of agent model. Moreover, sometimes the nature of knowledge processes is quite similar even if they have different names (e.g., knowledge creation and knowledge development). Therefore, the most common processes were chosen. Providing explicit feedback loops will make the model more oriented to implementation problems that could be associated with the interventions. Although this remark is accurate, in order to keep the model tractable for learning purposes, we deliberately assumed that interventions are effective. We agree that the utilisation of knowledge by a person and consequently the contribution of that person to the business outcome depends on the individual's level of competence. Since we modelled the 'organisational' level of competence and 'organisational' knowledge processes, not the personal, we decided to establish a separate influence of these variables on business outcomes. By doing this, we could also implement cases when, for example, changes in the organizational structure influence knowledge utilisation processes but might not affect the level of competence. Additionally, it was implemented that influences that immediately affect the level of competence in a knowledge domain influence knowledge utilisation with a small delay. For example, a training program immediately influences knowledge gaining and knowledge development, which both contribute to the level of competence. Consequently there is a positive but delayed effect on knowledge utilization. Additionally, experts commented that level of competence has an influence on knowledge processes. For this, in the model we realised a mechanism of threshold values for some variables. The level of competence is an example of a 'threshold' variable working as a feedback function for the whole situation in the company. When the level of competence has a high value, then 'problematic' events cannot occur in the company and when the level of competence is low, an event which describes miscommunication processes between employees or an event describing mistakes of employees, can be triggered by the system.

Taken together, the comments of the experts mainly refer to aspects that have to do either with the completeness of the model (missing domains and links) or with the scope of the model (individual vs. organisational, effectiveness of interventions). The former can lead to improvements in the model, but does not seriously jeopardize the representational validity of the model. The latter is correct, but would require another type of model; this is a question about the decision to select a particular scope for the model, rather than a judgment about validity, given the scope of the model.

\subsubsection{Interventions specifications}

In Table 7.4 summative statistics, which consists of the opinion of experts, for each intervention are presented. The specification of an intervention is considered to be valid if the majority of experts agreed with the specification. The specification of an intervention is considered invalid if half or more specified influences were considered by experts to be incorrect. When experts made a minor change in the specification, then her or his opinion was counted to be valid or invalid depending on how many experts agreed or disagreed with the specification. 
Minor changes are:

Type 1 - one change in influence (one influence added or deleted)

Type 2 - one change in timing of a specification

Type 3 - one change in persistence of an influence

Table 7.4 Summative statistics for interventions

\begin{tabular}{|c|c|c|c|c|c|c|c|c|}
\hline \multirow{2}{*}{ Interventions } & \multirow{2}{*}{$\mathrm{N}$} & \multirow{2}{*}{$\begin{array}{l}\text { Agree with } \\
\text { specification }\end{array}$} & \multicolumn{3}{|c|}{$\begin{array}{l}\text { Accept with minor } \\
\text { change }\end{array}$} & \multirow{2}{*}{$\begin{array}{c}\text { Reject } \\
\text { specifications }\end{array}$} & \multirow{2}{*}{ Valid \% } & \multirow{2}{*}{ Invalid \% } \\
\hline & & & Type 1 & Type 2 & Type 3 & & & \\
\hline Intervention 1 & 9 & 6 & 2 & 1 & & & 100 & 0 \\
\hline Intervention 2 & 9 & 4 & 1 & & & 4 & 55.6 & 44.4 \\
\hline Intervention 3 & 9 & 8 & 1 & & & & 100 & 0 \\
\hline Intervention 4 & 9 & 3 & 3 & 1 & & 2 & 77.8 & 22.2 \\
\hline Intervention 5 & 9 & 7 & & 2 & & & 100 & 0 \\
\hline Intervention 6 & 9 & 7 & & 1 & & 1 & 88.9 & 11.1 \\
\hline Intervention 7 & 9 & 7 & & & & 2 & 77.8 & 22.2 \\
\hline Intervention 8 & 9 & 5 & 2 & & & 2 & 77.8 & 22.2 \\
\hline Intervention 9 & 9 & 3 & & & 2 & 4 & 33.3 & 66.7 \\
\hline Intervention 10 & 9 & 6 & & 1 & & 2 & 77.8 & 22.2 \\
\hline
\end{tabular}

From the 10 randomly selected interventions, specifications of two interventions (intervention 2 and intervention 9) jeopardize the model's validity while the specifications of 8 interventions satisfy the validity requirements. Generalizing this result for the entire pool of interventions, it can be concluded that 80 percent of all interventions satisfy the validity requirements, which is quite acceptable. Average validity percentage for these 10 interventions reached a value of 78,9 percent which is a good result; therefore, we consider the model as possessing internal representational validity.

In the examples below, experts' opinions and comments for two interventions are given: intervention 3 - the majority of the experts agreed with the intervention specifications (See Example 7.2) and intervention 9 - the majority of experts rejected the intervention specifications (See Example 7.3). 
Example 7.2 Example of intervention 3 with specifications and experts' comments

\section{Intervention 3}

Contract marketing agencies to conduct a market research to learn customers' priorities and competitors' advances in products and services

\section{Specification}

This intervention leads to knowledge gaining in the marketing domain since the company gets the results of marketing research. This intervention has an immediate effect, which persist over short period.

Experts' specifications and comments

\begin{tabular}{|l|l|l|}
\hline Expert & Specifications & Comments from experts \\
\hline Expert 6 & $\begin{array}{l}\text { Additionally to the model } \\
\text { specifications positive } \\
\text { delayed influence on } \\
\text { knowledge utilisation in } \\
\text { all domains. }\end{array}$ & $\begin{array}{l}\text { This intervention has a delayed influence on the } \\
\text { utilisation process in all areas. Better } \\
\text { knowledge about the market leads (or should } \\
\text { lead) to better alignment of knowledge } \\
\text { resources and market needs. This goes beyond } \\
\text { the marketing department. Whether this is } \\
\text { short-term or long term, depends on the specific } \\
\text { situation. }\end{array}$ \\
\hline
\end{tabular}

The other experts agreed with the proposed specifications

Most of the experts' comments referred to the persistence of the intervention's influence or the effectiveness of the intervention for different knowledge domains. We agree that this intervention has a different influence on each knowledge domain. In the model this is provided for using different decay functions for knowledge processes for three knowledge domains. For example, the fastest decay for knowledge utilisation is in the R \& D knowledge domain, therefore the effect of this intervention will be shortest for this domain. On the other hand, since knowledge utilisation in Production has the slowest decay, the effect of this intervention will be longest for the Production knowledge domain. In addition, the magnitude of the influence differs for all knowledge domains. Since it was chosen to demonstrate a unified approach in the modelling of influences and there is no specific reference in the intervention description to a particular knowledge domain, the effect of this intervention will exist and will be positive for all knowledge domains. The longest influence that was modelled lasted 4 game periods, which is equal to one year. This intervention is possible to implement in the game only twice with an interval of 6 game periods. Moreover, in real life the nature of the bonus system is also different. For example, the bonus could be financial rewards as well as additional facilities for employees. Creating a model on very specific level will change the nature and goal of the model. For the purpose of a model which is to show how knowledge and the dynamism of knowledge processes influence organizational outcomes in general; a relatively abstract modelling level was chosen. 
Example 7.3 Example of the intervention 9 with specification and experts' comments

\author{
Intervention 9 \\ Implement a bonus system for employee effectiveness
}

\title{
Specification
}

This intervention has immediate positive long-term effect on knowledge retention and knowledge utilisation processes in all knowledge domains and additionally a positive influence on job satisfaction index since there is a rewarding policy (this last influence cannot be entered in the table).

Experts' specifications and comments

\begin{tabular}{|c|c|c|}
\hline Expert & Specifications & Comment from Expert \\
\hline Expert 1 & $\begin{array}{l}\text { Immediate positive } \\
\text { short-term influence on } \\
\text { utilisation and retention } \\
\text { in all domains. }\end{array}$ & $\begin{array}{l}\text { Financial rewards tend to work short-term and } \\
\text { not so effective in long term (e.g. salary rise } \\
\text { improves performance for about } 6 \text { months) }\end{array}$ \\
\hline Expert 2 & $\begin{array}{l}\text { Immediate negative } \\
\text { long-term influence on } \\
\text { development and } \\
\text { utilization in R\&D. } \\
\text { Immediate positive long- } \\
\text { term influence in } \\
\text { utilisation and retention } \\
\text { for Production. }\end{array}$ & $\begin{array}{l}\text { This one is very difficult: the effects are most } \\
\text { likely positive on all processes in production } \\
\text { and most likely negative on all processes in } \\
\text { R\&D (because of out crowding). All depends on } \\
\text { the nature and reception of the bonuses. }\end{array}$ \\
\hline Expert 3 & $\begin{array}{l}\text { Disagreed with the } \\
\text { model specifications }\end{array}$ & $\begin{array}{l}\text { The influences are short-term, depends on a } \\
\text { system }\end{array}$ \\
\hline Expert 4 & $\begin{array}{l}\text { Agreed with the model } \\
\text { specifications }\end{array}$ & \\
\hline Expert 5 & $\begin{array}{l}\text { Disagreed with the } \\
\text { model specifications }\end{array}$ & $\begin{array}{l}\text { No effect on retention (or very small). Might } \\
\text { attract new people (gaining) }\end{array}$ \\
\hline Expert 6 & $\begin{array}{l}\text { Disagreed with the } \\
\text { model specifications }\end{array}$ & $\begin{array}{l}\text { I do not believe that this intervention has a } \\
\text { (direct) effect on the effectiveness of knowledge } \\
\text { processes. If at all, they only work (very) short- } \\
\text { term. Reward systems are no satisfiers. They } \\
\text { can, however, be dissatisfiers. }\end{array}$ \\
\hline Expert 7 & $\begin{array}{l}\text { Disagreed with the } \\
\text { model specifications. }\end{array}$ & $\begin{array}{l}\text { Bonuses do not work. In case they work the } \\
\text { influence is not longer then } 1 \text { year until the next } \\
\text { appraisal talk. Even might work negatively after. }\end{array}$ \\
\hline Expert 8 & $\begin{array}{l}\text { Agreed with the model } \\
\text { specifications }\end{array}$ & \\
\hline Expert 9 & $\begin{array}{l}\text { Agreed with the model } \\
\text { specifications }\end{array}$ & \\
\hline
\end{tabular}

\subsubsection{The validity questionnaire}

As can be seen in Table 7.5, in most cases experts judged the model and its components as representatively valid. Most of the scores lie between 4 and 5 (slightly agree to agree). Two components reflecting internal representational validity are assessed above 5 (agree to strongly agree). These components concern the plausibility of knowledge domains and 
the knowledge processes in the game. Four components of the model are assessed below 4 (from slightly disagree to slightly agree): the plausibility and applicability of the knowledge process related variables, the applicability of interventions, and the credibility of the influence of interventions (Bold in Table 7.5).

Table 7.5 Descriptive statistics about experts' judgments on the validity propositions

\begin{tabular}{|l|c|c|c|c|}
\hline \multicolumn{1}{|c|}{ Model components } & $\mathrm{N}$ & $\begin{array}{c}\text { Plausibility } \\
\text { Min-Max, (SD) }\end{array}$ & $\begin{array}{c}\text { Applicability } \\
\text { Min-Max, (SD) }\end{array}$ & $\begin{array}{c}\text { Credibility } \\
\text { Min-Max, (SD) }\end{array}$ \\
\hline Conceptual model & $9^{*}$ & $\begin{array}{c}4.89 \\
4-6(.601)\end{array}$ & $\begin{array}{c}4.44 \\
-5(.726)\end{array}$ & $\begin{array}{c}4.25 \\
2-6(1.282)\end{array}$ \\
\hline Knowledge domains & $9^{*}$ & $\begin{array}{c}5.11 \\
4-6(.601)\end{array}$ & $\begin{array}{c}4.11 \\
2-6(1.167)\end{array}$ & $\begin{array}{c}4.88 \\
4-6(.641)\end{array}$ \\
\hline Knowledge processes & $9^{*}$ & $\begin{array}{c}5.33 \\
4-6(.707)\end{array}$ & $\begin{array}{c}4.67 \\
-5(.707)\end{array}$ & $\begin{array}{c}4.44 \\
4-5(.527)\end{array}$ \\
\hline The business process variables & 9 & $\begin{array}{c}4.78 \\
2-6(1.093)\end{array}$ & $\begin{array}{c}4.44 \\
2-5(1.130)\end{array}$ & $\begin{array}{c}4.5 \\
4(.535)\end{array}$ \\
\hline $\begin{array}{l}\text { The knowledge process related } \\
\text { variables }\end{array}$ & 9 & $\begin{array}{c}3.89 \\
2-6(1.453)\end{array}$ & $\begin{array}{c}3.89 \\
2-5(1.167)\end{array}$ & $\begin{array}{c}4.00 \\
2-5(1.000)\end{array}$ \\
\hline The interventions & 9 & $\begin{array}{c}4.33 \\
2-5(1.118)\end{array}$ & $\begin{array}{c}3.89 \\
2-5(1.054)\end{array}$ & $\begin{array}{c}4.33 \\
2-5(1.000)\end{array}$ \\
\hline The events & 9 & $\begin{array}{c}4.89 \\
4(.601)\end{array}$ & $\begin{array}{c}4.67 \\
3-6(.866)\end{array}$ & $\begin{array}{c}4.56 \\
2-5(1.014)\end{array}$ \\
\hline The decay behavior of the model & $9 *$ & $\begin{array}{c}4.63 \\
2-6(1.408)\end{array}$ & $\begin{array}{c}4.50 \\
2-6(1.414)\end{array}$ & $\begin{array}{c}4.38 \\
2-6(1.302)\end{array}$ \\
\hline The influence of interventions & 9 & $\begin{array}{c}4.44 \\
3-6(.882)\end{array}$ & $\begin{array}{c}4.56 \\
3-5(.726)\end{array}$ & $\begin{array}{c}3.67 \\
2-5(1.118)\end{array}$ \\
\hline The influence of events & 9 & $\begin{array}{c}4.89 \\
3-6(.928)\end{array}$ & $\begin{array}{c}4.56 \\
3-6(.882)\end{array}$ & $\begin{array}{c}4.00 \\
2-5(1.000)\end{array}$ \\
\hline The behavior of the model & 9 & $\begin{array}{c}4.89 \\
4-6(.782)\end{array}$ & $\begin{array}{c}4.67 \\
3-5(.707)\end{array}$ & $\begin{array}{c}4.11 \\
2-5(1.054)\end{array}$ \\
\hline
\end{tabular}

* - one expert did not mark the credibility proposition

For 14 validity propositions standard deviations are larger then one and 8 of them have a bimodal distribution. In all cases the bimodality is not located on the extreme ends of the scale and the distribution is skewed to the positive end of the scale.

Overall, from the 33 propositions, only 4 are assessed as being not representatively valid (from which one proposition concerns internal representational validity and three external representational validity), while the other 29 propositions (88 percent of all judgments) are assessed as being valid. More specifically internal representational validity reaches a value of 90,9 percent (one validity proposition out of eleven is not valid) and external representational validity reaches a value of 86,4 percent (three validity propositions out of 22 are considered invalid). This can be seen as confirming the representational validity of the model to a large extent. 


\subsection{Discussion and conclusion}

In this explorative study we investigated the representational validity of the model with experts in the knowledge management domain. We addressed external representational validity of the model by asking the experts to evaluate modelling assumptions and decisions which were made during the model building process and to give their opinion about these. As for internal representational validity, experts gave their opinions about the specifications for 10 randomly selected interventions, which are part of the model logic and structure. Additionally experts judged internal and external representational validity of the model by giving their opinion on the validity propositions concerning the model and its components.

Although experts commented that they would like to have additional knowledge domains, and knowledge processes incorporated into the model, and have a more detailed model, experts judged all modelling assumptions and decisions positively. In percentages external representational validity of the model was assessed by experts as being equal to 80 percent.

Internal representational validity as a result of experts' evaluation of specification of interventions influences was assessed as being equal to 78,9 percent. While 80 percent of interventions satisfy the validity requirements, 20 percent of interventions are considered by experts as not being properly specified. Experts' opinions differ from each other in terms of what kind of specifications particular interventions should have.

In the validity questionnaire experts judged four validity propositions as invalid. Three of them refer to the external representational validity and one to the internal representational validity. Overall, judgments on the validity propositions in the validity questionnaire confirmed that the model possesses internal representational validity to the extent of 90,9 percent and external representational validity to the extent of 86,4 percent.

These results show that the model possesses representational validity to a large degree.

Measuring validity in percentages brings us to an interesting point in discussion: when is a model valid? Can we provide an absolute 100 percent validity? Obviously, when we model an abstract phenomenon in which the relations between elements in the reference system are unknown and do not have a clear structure, we have to adjust our ambition to reach absolute validity. What degree of validity should the model possess? To answer this question we have to look back on our discussion about physical and functional fidelity, which the model needs to possess in order to support learning about the modelled phenomenon. When we need to teach operational skills then the model needs to possess a high level of physical fidelity, while a high level of functional fidelity is desirable when teaching intuitive managerial skills. In this case functional fidelity is the degree to which a model reproduces functional characteristics and behaviour of a real world object. In the KM Quest learning environment the real world object is an abstract Product leadership company in which business outcomes depend on the level of knowledge and effectiveness of knowledge processes in the company. To formalise knowledge processes we introduced the knowledge process related variables. Although experts judged the knowledge process related variables as invalid, the presence of these variables provides a higher educational validity of the model what was proven in the first experimental study (See Chapter 5). At the same time different levels of physical and functional fidelity influenced the perception of the model as a valid representation of 
reality by players of the game but did not influence the model's educational validity. Hence, presence of the knowledge process related variables in the model supports the model's educational validity but jeopardize its representational validity.

Another point of discussion refers to the fact that every expert has his or her own ideas about knowledge domains, knowledge processes and the relationships between them. This shows how difficult it is to model an abstract phenomenon and that many agreements and choices should be made during the modelling process in order to simplify reality in a way that the model does not become too difficult and complex yet still suits purposes it is developed for - (reflecting the phenomenon and its characteristics). In this sense, a perfect model does not exist.

Overall, given the diversity of experts' judgments and opinions, it can be concluded that the modelling decisions made for our model still can count on the endorsement of the majority of the experts involved in this study. Though this does not make the model the ultimate "right" model, it increases the confidence that this model captures the essential features of knowledge management in organizations. 


\section{Chapter 8}

\section{Across the studies}

In this chapter we compare results of the previous studies. Here we look at how the different model representations affected the game performance of the players (which is a particular case of internal educational validity) and the judgments of the players on the internal and external representational validity of the model. Additionally, we compare game performance and judgments of the representational validity made by students and experts. 


\subsection{Introduction}

In the previous chapters we described several studies investigating how different levels of the model's fidelity and the model's complexity influenced the internal and external educational validity of the model. In these studies, fidelity was varied by presenting a different model representation to the players and complexity was varied by altering the amount of observable variables and by altering whether or not support was provided in decision making. Additionally, we examined how players judged the model's internal and external representational validity. In this chapter we will compare the results of these experiments in order to find out which model, in terms of possessing different levels of fidelity and complexity, provides a higher internal and external educational validity and internal and external representational validity. In addition, we will investigate if there are relations between educational and representational validity: does an optimal combination of perceived representational validity and educational validity exist? Finally, we will examine how perceived representational validity judged by students who played the game, differs from representational validity as assessed by experts in the KM domain. This will help us to identify the model's validity "bottlenecks", if any, in order to find out where students need more support in order to prevent perceived representational validity from negatively influencing the model's educational validity. Answers to these questions will help us to identify which model is more efficient in terms of providing higher educational validity, which is important for KM Quest as an educational game. This investigation has an exploratory character since in this uncharted territory there is no theory from which hypotheses can be derived.

In the first section of this chapter we assess how differences in the fidelity level of the game simulation model and differences in the availability of the supportive KM model, which guides players to choose interventions, influenced the game performance of the players. We examine how this could affect their judgments about the external and internal representational validity of the model. In a comparative analysis we will use only data from individual players in order to make sure that no other factors (e.g, mode of playing) except the model's features and the model's support, influence game performance and judgments about the model's validity. Therefore, the data from collaborative players in the second experiment are left deliberately out of this analysis.

In the third study we described how a group of experts knowledgeable in the area of knowledge management assessed the model's modeling propositions based on their work experience and the model's validity propositions based on their KM Quest game experience. In the second section of this chapter we compare game performance and judgments of experts with the group of players from the first study in order to investigate how the perceived representational validity differs from the representational validity and how the experts' game performance differs from the students' game performance. In order to make a comparison credible, experts played the game with all variables available, but had no access to the supportive KM model. In other words, the experts' game and model environment was equal to the group of players from the first study, which had the knowledge process variables available but not the supportive KM model. 
Differences in the model's representation and in the model's support could have an influence on the construction of conceptual and strategic knowledge, which makes a comparison of the knowledge test scores incompatible. Therefore, we limit this chapter to comparing only game performance indicators that refer to the internal educational validity, and to comparing judgments on internal and external representational validity.

\subsection{Comparison between students' game results and students' validity judgments}

\subsubsection{Differences in the model representation and the game environment}

For the purpose of finding out how differences in the model's representation can affect game performance and judgments on representational validity, we compared game results and judgments on the representational validity between three groups: two groups of individual players from the first study and the group of individual players in the second study. For these three groups the following differences in the game environment and in the model representation apply (see Table 8.1). For simplicity reasons, we will name these groups as follows:

Condition 1 - individual players from the first experiment who had access to the knowledge process related variables, but did not have support from the supportive KM model;

Condition 2 - individual players from the first experiment, who did not have access to the knowledge process related variables and to the supportive KM model;

Condition 3 - individual players from the second experiment, who did have access to the knowledge process related variables and to the supportive KM model, but did not have access to the business process related variables.

Table 8.1 Differences in the game environment and in the model representation

\begin{tabular}{|l|c|c|c|}
\hline $\begin{array}{c}\text { The game } \\
\text { environment and the } \\
\text { model components }\end{array}$ & $\begin{array}{c}\text { Condition 1 } \\
\text { The group of individual } \\
\text { players - Experiment 1 }\end{array}$ & $\begin{array}{c}\text { Condition 2 } \\
\text { The group of individual } \\
\text { players - Experiment 1 }\end{array}$ & $\begin{array}{c}\text { Condition 3 } \\
\text { The group of individual } \\
\text { players - Experiment 2 }\end{array}$ \\
\hline $\begin{array}{l}\text { Advice and } \\
\text { visualization }\end{array}$ & Not available & Not available & Not available \\
\hline Feedback & Available & Available & Available \\
\hline $\begin{array}{l}\text { Supportive KM } \\
\text { model }\end{array}$ & Not accessible & Not accessible & Accessible \\
\hline $\begin{array}{l}\text { Knowledge process } \\
\text { related variables }\end{array}$ & $\begin{array}{c}\text { Visible and accessible } \\
\text { for players }\end{array}$ & $\begin{array}{c}\text { Not visible and not } \\
\text { accessible for players }\end{array}$ & $\begin{array}{c}\text { Visible and accessible } \\
\text { for players }\end{array}$ \\
\hline Knowledge variables & $\begin{array}{c}\text { Visible and accessible } \\
\text { for players }\end{array}$ & $\begin{array}{c}\text { Visible and accessible } \\
\text { for players }\end{array}$ & $\begin{array}{c}\text { Visible and accessible } \\
\text { for players }\end{array}$ \\
\hline $\begin{array}{l}\text { Business process } \\
\text { related variables }\end{array}$ & $\begin{array}{c}\text { Visible and accessible } \\
\text { for players }\end{array}$ & $\begin{array}{c}\text { Visible and accessible } \\
\text { for players }\end{array}$ & $\begin{array}{c}\text { Not visible and not } \\
\text { accessible for players }\end{array}$ \\
\hline $\begin{array}{l}\text { Organizational } \\
\text { effectiveness } \\
\text { variables }\end{array}$ & $\begin{array}{c}\text { Visible and accessible } \\
\text { for players }\end{array}$ & $\begin{array}{c}\text { Visible and accessible } \\
\text { for players }\end{array}$ & $\begin{array}{c}\text { Visible and accessible } \\
\text { for players }\end{array}$ \\
\hline
\end{tabular}


From the model's fidelity point of view, the model presented to the players in Condition 1 possessed a higher level of functional fidelity and a moderate level of physical fidelity. The model presented to the players in Condition 2 possessed a higher level of physical fidelity and a lower level of functional fidelity. The model presented to the players in Condition 3, possessed a low level of physical fidelity and a moderate level of functional fidelity. From the model's complexity point of view (by complexity we mean the amount of variables to observe and the number of decisions to be taken, see discussion in Chapter 4), the most complex was the model presented for Condition 1 because players observed more unfamiliar variables and made their decisions without assistance of the supportive KM model. Moderate complexity was possessed by the model presented for Condition 2: the number of variables to observe was the smallest, but the supportive KM model which assists in the process of taking decisions was not available. The least complex model for the players was the model in Condition 3: the supportive KM model supported players and the number of variables to observe was a level between the other two conditions (see Table 8.2).

Table 8.2 Fidelity and complexity of the model for the three conditions

\begin{tabular}{|l|l|l|l|}
\hline \multirow{2}{*}{ Condition } & \multicolumn{2}{|c|}{ Fidelity } & \multirow{2}{*}{ Complexity } \\
\cline { 2 - 3 } & Physical & Functional & \\
\hline Condition 1 & middle & high & high \\
\hline Condition 2 & high & low & middle \\
\hline Condition 3 & low & middle & low \\
\hline
\end{tabular}

All games were identical in terms of the sequence and order of events; the initial values of variables and the model behavior were the same in all games. The differences existing between conditions were in the area of model representation for the players and in the availability of additional support. All players had no experience with the game, therefore the data about game performance and the judgments of players on the representational validity are comparable.

\subsubsection{Results of the cross-experimental analysis}

\subsubsection{Internal educational validity: game performance}

In our studies game performance reflects internal educational validity of the model and is measured via three performance indicators: Profit, $\mathrm{K}$-value and $\mathrm{N}$-number of interventions. Table 8.3 provides an overview of the three performance measures for the three conditions.

A one-way ANOVA with Condition as the independent factor showed that there is a significant difference between conditions on the Profit $(\mathrm{F}(2,69)=6.67, \mathrm{p}=.002)$ and the Kvalue indicator $(\mathrm{F}(2,69)=4.85, \mathrm{p}=.011)$. There was no significant difference on the $\mathrm{N}$ indicator, although the group with the supportive KM model available (Condition 3 ) achieved the highest scores on the profit indicator by implementing the lowest number of interventions. 
Table 8.3 Means and standard deviations of three performance measures

\begin{tabular}{|l|l|l|l|l|}
\hline Measure & Condition & N & Mean & St. deviation \\
\hline \multirow{4}{*}{ Profit } & Condition 1 & 20 & 72480905.70 & 15362168.058 \\
\cline { 2 - 5 } & Condition 2 & 20 & 67026065.40 & 12551925.428 \\
\cline { 2 - 5 } & Condition 3 & 32 & 79998804.12 & 10955241.308 \\
\hline $\mathbf{N}$ & Condition 1 & 20 & 43.75 & 8.403 \\
\cline { 2 - 5 } & Condition 2 & 20 & 41.80 & 9.260 \\
\cline { 2 - 5 } & Condition 3 & 32 & 37.72 & 11.28 \\
\hline $\mathbf{K}$ & Condition 1 & 20 & 24.0350 & 2.83432 \\
\cline { 2 - 5 } & Condition 2 & 20 & 21.1065 & 2.45411 \\
\cline { 2 - 5 } & Condition 3 & 32 & 24.1341 & 4.58906 \\
\hline
\end{tabular}

A Tukey HSD post-hoc test showed three significant differences out of six possible on the Profit and the K-value indicators between the groups. There was a significant difference on the Profit indicator between the group without the knowledge process related variables (Condition 2) and group with the knowledge process related variables and supportive KM model available (Condition 3) $(\mathrm{p}=.002)$ in favor of the latter. At the same time, the post-hoc test revealed that there were significant differences on the Kvalue indicator between the group without knowledge process related variables (Condition 2) and the group with knowledge process related variables and with the supportive KM model not available (Condition 1) $(\mathrm{p}=.035)$. There were additionally differences between the group without knowledge process related variables (Condition 2) and the group with knowledge process related variables and an available supportive KM model (Condition 3) $(\mathrm{p}=.013)$. For both instances there were lower values for the group without knowledge process related variables accessible (Condition 2). Thus Condition $2-$ the group without knowledge process related variables - showed the least satisfactory results. No significant differences were found between Condition 1 and Condition 3 on the performance indicators. Although we did not find significant differences in performance indicators between groups with knowledge process variables and who differed in the availability of the supportive KM model, the group with the available supportive KM model achieved slightly higher scores on the Profit and K-value indicators by applying fewer interventions.

These data show that the model which included knowledge process related variables and an available supportive KM model or, in other words, the model with a low level of physical fidelity, a moderate level of functional fidelity and a low level of complexity (Condition 3) is associated with a higher internal educational validity when compared with the other models. The model with a lower level of functional fidelity and a moderate level of complexity (Condition 2) provided the lowest internal educational validity. The model with a high level of functional fidelity and complexity and a moderate level of physical fidelity (Condition 1) gave almost the same results as the model in Condition 3. These conditions both included the knowledge process related variables but differed in the accessibility of the business process related variables and the model support - the guidance of players in the acquisition of conceptual knowledge with help of the supportive KM model. 
In the first study we found that the model with accessible knowledge process variables (Condition 1 here) provided a higher internal and external educational validity than the model without access to these variables (Condition 2 here). At the same time, the second study showed that the model without business process variables available (Condition 3 here) did not lead to acquisition of conceptual knowledge by individual players, which is also the part of internal educational validity. Therefore, we suggest that a model which will combine the features of the models and the model's support from Condition 1 and Condition 3 will provide higher levels of internal and external educational validity. In other words, the ideal model will possess a high level of functional fidelity and moderate level of complexity (e.g. a model containing all variables and the supportive KM model available).

\subsubsection{Perceived representational validity}

Perceived representational validity is assessed by Plausibility as a measure of perceived internal representational validity and by Applicability and Credibility as measures of perceived external representational validity. It is important to know that players in Condition 1 had access to and could observe knowledge process related variables in the model, players in Condition 2 had no access to and could not observe knowledge process related variables and players in Condition 3 had no access to and could not observe the business process related variables. Players who could not observe different variables in the model, still gave their judgments on these variables (see model components: the business process variables and the knowledge process variables in Table 8.4). Table 8.4 presents an overview of the mean judgment scores and standard deviations (SD) for the representational validity propositions of the three conditions (two players from Condition 3 did not fill in the validity questionnaire and are not included in the analysis).

From a complete set of 99 validity judgments of players in the three conditions, only 12 validity judgments are below 4 (from slightly disagree to slightly agree), 86 judgments are above 4 (from slightly agree to agree) and one validity judgment is equal to 5 (agree). In the Plausibility category 31 judgments are above 4 and two are below 4; in the Applicability category 32 judgments are above 4 and one is below 4 , and in the Credibility category 24 judgments are above 4 and 9 are below 4 . This shows that the model's representational validity is perceived positively overall by the players. Lower scores of validity propositions in the Credibility category could be explained by the fact that players in Condition 1 and in Condition 3 (only these players gave scores below 4) had to work with the model which included knowledge process related variables which are not used in reality and are not familiar to them.

A one-way ANOVA revealed that there are significant differences between conditions on the following validity proposition items:

- The plausibility of the decay behavior of the model $(\mathrm{F}(2,67)=3.316, \mathrm{p}=.042)$;

- The applicability of the knowledge process related variables $(\mathrm{F}(2,67)=4.577$, $\mathrm{p}=.014$ )

- The applicability of the decay behavior of the model $(\mathrm{F}(2,67)=4.581, \mathrm{p}=.014)$ 
- The credibility of the conceptual model $(\mathrm{F}(2,67)=5.170, \mathrm{p}=.008)$

- The credibility of the knowledge process related variables $(F(2,67)=5.764$, $\mathrm{p}=.005$ )

There is a significant difference between conditions on the overall Credibility category $(\mathrm{F}(2,67)=5.741, \mathrm{p}=.005)$

A post hoc analysis (Tukey HSD) showed that these differences are significant for the following groups:

- The plausibility of the decay behavior of the model is significantly different between players in Condition 1 and Condition 2. The higher scores were given by the players who did not have access to the knowledge process related variables (Condition 2) $(\mathrm{p}=.037)$

- The applicability of the knowledge process related variables of the players who did not have access to the knowledge process related variables and did not have the supportive KM model available (Condition 2) was rated significantly higher than by players who had access to the knowledge process related variables but did not have the supportive KM model available (Condition 1) $(\mathrm{p}=.014)$

- The applicability of the decay behavior of the model judged by players who had access to the knowledge process related variables and to the supportive KM model (Condition 3) significantly lower than by players who did not have access to the knowledge process related variables (Condition 2$)(\mathrm{p}=.011)$

- The credibility of the conceptual model assessed by players who had access to the knowledge process related variables and to the supportive KM model (condition 3) significantly lower than by players who did not have access to the knowledge process related variables (Condition 2) $(\mathrm{p}=.006)$;

- The credibility of the knowledge process related variables was judged by players who had no access to the knowledge process related variables (Condition 2) as being more credible than by both players who had access to the knowledge process related variables, but did not have access to the supportive KM model (Condition 1) $(\mathrm{p}=.005)$ and players who did have access to the knowledge process related variables and to the supportive KM model (Condition 3) ( $\mathrm{p}=.037)$.

The overall credibility was judged to be significantly higher by players who could not observe knowledge process related variables (Condition 2) than by players who could observe knowledge process related variables and had support from the supportive KM model but could not observe business process related variables (Condition 3 ).

There were no significant differences found between judgments of players in Conditions 1 and 3 on internal and external representational validity.

These data show that players who played the game with the model possessing a high level of physical fidelity, a low level of functional fidelity and a moderate level of complexity (Condition 2) assessed the model as being more representationally valid than the players in the other two conditions.

Although they could not observe them, players from Condition 2 assessed the plausibility of the decay behavior of the model, the applicability and the credibility of the knowledge process related variables more positively than players from Condition 1 who worked with 
the model possessing a high level of functional fidelity, a moderate level of physical fidelity and a high level of complexity.

Table 8.4 Mean judgment scores and standard deviations (SD) on the representational validity items for three conditions.

\begin{tabular}{|c|c|c|c|c|c|}
\hline $\begin{array}{l}\text { Model } \\
\text { components }\end{array}$ & Condition & $\mathbf{N}$ & Plausibility & Applicability & Credibility \\
\hline \multirow{3}{*}{ Conceptual model } & Condition 1 & 20 & $4.20(1.056)$ & $4.55(.945)$ & $3.90(.788)$ \\
\hline & Condition 2 & 20 & $4.50(1.147)$ & $4.85(.587)$ & $4.50(607)$ \\
\hline & Condition 3 & 30 & $4.67(.844)$ & $4.47(.730)$ & $3.70(1.05)$ \\
\hline \multirow{3}{*}{ Knowledge domains } & Condition 1 & 20 & $4.55(.887)$ & $4.45(.826)$ & $4.65(.988)$ \\
\hline & Condition 2 & 20 & $4.40(1.095)$ & $4.35(.745)$ & $4.45(.686)$ \\
\hline & Condition 3 & 30 & $4.40(.894)$ & $4.27(.785)$ & $4.10(.885)$ \\
\hline \multirow{3}{*}{$\begin{array}{l}\text { Knowledge } \\
\text { processes }\end{array}$} & Condition 1 & 20 & $3.90(1.210)$ & $4.45(.605)$ & $4.50(1.00)$ \\
\hline & Condition 2 & 20 & $4.35(1.040)$ & $4.65(.587)$ & $4.50(.761)$ \\
\hline & Condition 3 & 30 & $4.53(.937)$ & $4.40(.770)$ & $4.20(714)$ \\
\hline \multirow{3}{*}{$\begin{array}{l}\text { The business } \\
\text { process variables }\end{array}$} & Condition 1 & 20 & $4.60(.995)$ & $4.65(.933)$ & $4.65(.875)$ \\
\hline & Condition 2 & 20 & $4.70(1.031)$ & $4.75(.851)$ & $4.65(.875)$ \\
\hline & Condition 3 & 30 & $4.03(1.13)$ & $4.30(.952)$ & $4.20(1.03)$ \\
\hline \multirow{3}{*}{$\begin{array}{l}\text { The knowledge } \\
\text { process related } \\
\text { variables }\end{array}$} & Condition 1 & 20 & $4.15(1.089)$ & $3.75(1.118)$ & $3.55(.945)$ \\
\hline & Condition 2 & 20 & $4.35(.988)$ & $4.65(.875)$ & $4.50(.827)$ \\
\hline & Condition 3 & 30 & $4.40(1.00)$ & $4.00(.947)$ & $3.83(.950)$ \\
\hline \multirow{3}{*}{ The interventions } & Condition 1 & 20 & $4.15(.988)$ & $4.20(1.281)$ & $4.30(.801)$ \\
\hline & Condition 2 & 20 & $4.70(1.218)$ & $4.70(1.031)$ & $4.35(1.182)$ \\
\hline & Condition 3 & 30 & $4.33(1.02)$ & $4.33(.922)$ & $4.17(1.02)$ \\
\hline \multirow{3}{*}{ The events } & Condition 1 & 20 & $4.30(1.218)$ & $4.45(1.146)$ & $4.35(1.089)$ \\
\hline & Condition 2 & 20 & $4.45(1.191)$ & $4.75(.910)$ & $4.35(.933)$ \\
\hline & Condition 3 & 30 & $4.30(.877)$ & $4.53(.900)$ & $4.50(.974)$ \\
\hline \multirow{3}{*}{$\begin{array}{l}\text { The decay behavior } \\
\text { of the model }\end{array}$} & Condition 1 & 20 & $4.15(.933)$ & $4.70(.733)$ & $4.75(.786)$ \\
\hline & Condition 2 & 20 & $4.80(.834)$ & $5.00(.725)$ & $4.70(.979)$ \\
\hline & Condition 3 & 30 & $4.37(.718)$ & $4.30(.915)$ & $4.20(.887)$ \\
\hline \multirow{3}{*}{$\begin{array}{l}\text { The influence of } \\
\text { interventions }\end{array}$} & Condition 1 & 20 & $3.40(1.392)$ & $4.30(.657)$ & $3.85(.813)$ \\
\hline & Condition 2 & 20 & $4.10(1.165)$ & $4.10(.968)$ & $4.00(1.124)$ \\
\hline & Condition 3 & 30 & $4.07(1.02)$ & $4.13(1.01)$ & $3.90(1.09)$ \\
\hline \multirow{3}{*}{$\begin{array}{l}\text { The influence of } \\
\text { events }\end{array}$} & Condition 1 & 20 & 4.05 (1.234) & $4.15(.745)$ & $3.90(1.021)$ \\
\hline & Condition 2 & 20 & $4.10(1.518)$ & $4.60(.995)$ & $4.05(.945)$ \\
\hline & Condition 3 & 30 & $4.23(1.01)$ & $4.27(.785)$ & 3.97 (.999) \\
\hline \multirow{3}{*}{$\begin{array}{l}\text { The behavior of the } \\
\text { model }\end{array}$} & Condition 1 & 20 & $4.15(.875)$ & $4.15(.745)$ & $4.00(.858)$ \\
\hline & Condition 2 & 20 & $4.10(1.021)$ & $4.35(.813)$ & $4.25(.851)$ \\
\hline & Condition 3 & 30 & $4.27(.785)$ & $4.20(.847)$ & $3.97(.928)$ \\
\hline \multirow{3}{*}{ Aggregation } & Condition 1 & 20 & $45.60(5.413)$ & $46.40(5.051)$ & $47.80(5.634)$ \\
\hline & \begin{tabular}{|l|} 
Condition 2 \\
\end{tabular} & 20 & 48.55 (8.127) & $48.30(5.686)$ & $50.75(5.514)$ \\
\hline & Condition 3 & 30 & $47.60(5.14)$ & $47.20(5.67)$ & $44.73(7.24)$ \\
\hline
\end{tabular}


Players from Condition 2 assessed the applicability of the decay behavior of the model, the credibility of the conceptual model, the credibility of the knowledge process related variables and the overall credibility more positively than the players who worked with a model possessing a low level of physical fidelity, a low level of complexity and a moderate level of functional fidelity (Condition 3).

Overall, the judgments of the validity propositions revealed that players who did not have access to and could not observe the knowledge process related variables (Condition 2) gave scores above 4 (from slightly agree to agree) for all validity propositions for all categories. These players worked with the model that possessed a higher level of physical fidelity; on the aggregate level they assessed plausibility, applicability and credibility of the model higher than the players in the other two conditions.

From the plausibility items, players who had access to the knowledge process variables but did not have support from the supportive KM model (Condition 1) assessed only two propositions below 4 (from slightly disagree to slightly agree). These plausibility propositions refer to the plausibility of knowledge processes and the plausibility of the influence of interventions.

From all applicability items only one proposition was assessed below 4 (from slightly disagree to slightly agree) by players who had access and could observe knowledge process related variables, but did not have the supportive KM model available (Condition 1): the applicability of the knowledge process related variables. The group which had access to the knowledge process related variables and had the supportive KM model available (Condition 3), assessed these plausibility items and applicability items more positively than the other two groups.

Both groups, which had accessible and observable knowledge process related variables (Condition 1 and Condition 3) gave scores below 4 for four credibility validity propositions: the credibility of the conceptual model, the credibility of the knowledge process related variables, the credibility of influence of interventions, and the credibility of influence of events. The players who had access to the knowledge process related variables and the supportive KM model available (Condition 3) gave scores lower than 4 for the credibility of the behavior of the model.

On the aggregate level, the group which had access to and could observe knowledge process related variables but did not have support from the supportive KM model (Condition 1), assessed the plausibility of the model lower than the group that had access to the knowledge process related variables and support from the supportive KM model (Condition 3). At the same time the latter group gave the lowest scores on overall credibility in comparison to the other two groups. This is logical because the model presented to them had a lower physical fidelity since the business process variables were inaccessible and unobservable.

The results of the comparative analysis allow us to conclude that (1) presence of the knowledge process related variables, which decrease physical fidelity, has a negative influence on judgments on the internal and external representational validity; (2) the availability of the supportive KM model, which increases functional fidelity and decreases the model's complexity, positively influences judgments on the plausibility and applicability validity propositions; and (3) the model with the lowest level of physical 
fidelity (without the business process related variables) is assessed as being less credible. Hence, the model with a high level of physical fidelity, a low level of functional fidelity and a moderate level of complexity provides a higher perceived internal and external representational validity compared to other two possible implementations.

\subsection{Comparing student's and expert's game results and validity judgments}

\subsubsection{A special case of educational validity}

In Chapter 4 we described several approaches to measure internal and external educational validity. Of these approaches, Faria and Wellington (2005) mentioned that a simulation game is externally educationally valid if a successful real-world business executive is also successful when participating in the simulation and, for the internal educational validity of the game, a game is valid if better students outperform poorer students.

Since our research design for the first study was not aimed at assessing learning and learning results in the sense of knowledge gains on a pre- and post-test, we do not have records about the learning gains of students and cannot divide them into better or poorer learners. At the same time we do not have a measure for the experts' success. Therefore, we cannot check external educational validity and must focus on the internal educational validity, which we will investigate by comparing experts' and students' games results: if experts outperform students then the game simulation model possesses internal educational validity. To make the comparison more reliable and fair (experts are a priori more knowledgeable than students and we could easily overestimate internal educational validity by comparing 'poor' student performance with expert performance) we compare experts' games with the games of the students who achieved above average scores on the performance indicators.

\subsubsection{Perceived representational validity and representational validity}

In Chapter 4 (see Section 4.2.2.2), we stated that judgments on validity should be made by domain-experts, while players or users of the game judge the perception of validity.

Since experts and students played with similar games, that is the same model and environment condition for experts as for students in the first study (Condition 1), we will look how perceived representational validity (judgments of students) differs from representational validity (judgments of domain experts). There are three possible outcomes: (1) perceived representational validity and representational validity are judged similarly, (2) perceived representational validity is judged higher than representational validity, and (3) perceived representational validity is judged lower than representational validity. The first two cases do not have consequences for the educational validity while the third can have a negative influence, because the acceptance of the game model as a valid representation influences playing behavior and learning results (Moizer, Lean, Towler \& Smith, 2006). Moreover, as we decompose the judgments of the models' validity into several components of the model, the differences for a specific component can indicate a "bottleneck" of validity, or a component which should be supported more extensively for players ("learners" as we talk about educational validity). An example is 
providing more support for features of the game environment or a more elaborate instructional briefing.

\subsubsection{Results of the analysis}

\subsubsection{Educational validity of the model}

For testing the educational validity of the model we selected games played by students from the first experiment, Condition 1, who achieved scores above average on the performance indicators. Ten student games met this criterion. From the data of 9 experts who participated in the experts study, we used the games of experts who do not use the KM Quest game in their practice. For the purpose of this analysis, experts who use KM Quest in their practice are too familiar with the game and "winning" strategies, which could make the comparison unfavorable for students who had no experience with the game before they participated in the experiments. One of the experts overspent the game budget and was also excluded from analysis. This resulted in 6 expert games. Table 8.5 provides the mean values and standard deviations of three performance indicators, used in this research to measure internal educational validity: Profit, $\mathrm{K}$-value and $\mathrm{N}$ - number of interventions. As we stated in previous experimental studies, a good performance in the game means achieving high values of Profit and K-indicator while applying fewer interventions when compared with the standard: 32-40 interventions for 8 game periods.

Table 8.5 Mean values and standard deviations of three performance measures for experts and students conditions

\begin{tabular}{|l|l|l|l|l|}
\hline Measure & Condition & N & Mean & Std. Deviation \\
\hline \multirow{3}{*}{ Profit } & Students & 10 & 82519295.80 & 5967163.002 \\
\cline { 2 - 5 } & Experts & 6 & 81796915.50 & 10800528.89 \\
\hline \multirow{3}{*}{ K } & Students & 10 & 25.8210 & 1.28380 \\
\cline { 2 - 5 } & Experts & 6 & 24.4567 & 2.53025 \\
\hline \multirow{2}{*}{ N } & Students & 10 & 44.80 & 8.324 \\
\cline { 2 - 5 } & Experts & 6 & 31.50 & 6.285 \\
\hline
\end{tabular}

To find the differences between two conditions we used a t-test for independent samples. Although the standard deviations of two groups do differ, Levene's test showed that the variances in the two groups do not differ significantly. The t-test for independent samples showed that there are no significant differences in game performance between students and experts on the Profit and the K-value indicators ( $p>0.05$ ).

However, there is a significant difference between students and experts on the $\mathrm{N}$ indicator $(\mathrm{t}(14)=3.363, \mathrm{p}=.005)$. Experts achieved the same values on the Profit and the $\mathrm{K}$-value indicators by applying significantly fewer interventions. The absence of differences in the values of the Profit and the K-value indicators lies in the model construction and propagation of effects of interventions: first, there is a ceiling effect in the simulation (there are limits on company expansion in terms of market share and profit 
values in the game period) and secondly, students by applying more interventions, gave stronger impulses to change the situation. In later game periods, students will not be able to implement more interventions because they spent almost all their game budget in the first game periods and therefore some interventions will be not possible to implement anymore. Hence, the situation will change in the favor of experts, who still have money from the game budget and more possibilities to implement interventions. From this perspective we conclude, that the game simulation model possesses internal educational validity, because experts outperformed students as proposed by Faria and Wellington (2005).

\subsubsection{Perceived representational validity and representational validity of the model}

To compare judgments of representational validity, we take judgments given by all experts and judgments of students who played above average and below average. Such a comparison was based on two assumptions. First of all, we assume that students who played above average thought about the model more deeply and, as Goldstein \& Blackman (1978) believe, students who perform well in a game, possess to a higher extent meta-cognitive or control skills, therefore, they can think or reason as experts. This makes the comparison of mean judgments scores for validity propositions of students who performed above average and experts fair. Secondly, we cannot compare the mean judgments scores of students who played above (Students A) and below average (Students B), because these students were in the same condition and got the same treatment. Therefore, we compare the mean judgment scores of these two sub-groups against the third 'party' (Experts) in order to find the differences in judgments. This comparison allows us (1) to see whether the acceptance of the model as a valid representation of reality influenced the playing behavior of students and (2) to find validity 'bottlenecks'. We consider a validity 'bottleneck' to be a situation when scores of judgments on a validity propositions given by students are lower than scores of judgments given by experts, or, in other words, a situation when perceived representational validity is lower than representational validity. In such a situation the perceived representational validity of the model can negatively influence the educational validity of the model (Moizer, Lean, Towler \& Smith, 2006).

We can conclude that the perceived representational validity influences the game performance if the following holds true:

- The two-subgroups will not have the same significant differences in mean judgments scores as a result of comparisons between judgments of each sub-group and experts;

- We will find more significant differences between judgments of Students B and experts, than between judgments of Students A and experts; and

- The mean judgments scores of Students B will be lower than the mean judgments scores of experts.

To find the differences in judgments on perceived representational validity and representational validity we conducted two t-tests for independent samples and compared the mean judgment scores of students who performed above average (Students A in Table 8.6) and the mean judgment scores of experts as well as mean judgment scores of 
students who performed below average (Students $B$ in the Table 8.6) and mean judgments scores of experts.

The mean judgment scores and standard deviations for students and experts are displayed in Table 8.6.

Table 8.6 Mean judgment scores and standard deviations (SD) on the representational validity propositions for experts and students

\begin{tabular}{|c|c|c|c|c|c|}
\hline Model components & Condition & $\mathrm{N}$ & Plausibility & Applicability & Credibility \\
\hline \multirow[b]{3}{*}{ Conceptual model } & Students A & 10 & $4.00(1.155)$ & $4.30(1.059)$ & $3.70(.949)$ \\
\hline & Students B & 10 & $4.40(.966)$ & $4.80(.789)$ & $4.10(.568)$ \\
\hline & Experts & 9 & $4.89(.601)$ & $4.44(.726)$ & $4.25(1.282)$ \\
\hline \multirow[b]{3}{*}{ Knowledge domains } & Students A & 10 & $4.60(.699)$ & $4.30(.823)$ & $4.40(.966)$ \\
\hline & Students B & 10 & $4.50(1.080)$ & $4.60(.843)$ & $4.90(.994)$ \\
\hline & Experts & 9 & $5.11(.601)$ & $4.11(1.167)$ & $4.88(.641)$ \\
\hline \multirow[b]{3}{*}{ Knowledge processes } & Students A & 10 & $4.30(1.059)$ & $4.50(.527)$ & $4.30(1.160)$ \\
\hline & Students B & 10 & $3.50(1.269)$ & $4.40(.699)$ & $4.70(.823)$ \\
\hline & Experts & 9 & $5.33(.707)$ & $4.67(.707)$ & $4.44(.527)$ \\
\hline \multirow{3}{*}{$\begin{array}{l}\text { The business process } \\
\text { variables }\end{array}$} & Students A & 10 & $4.70(.823)$ & $4.70(.949)$ & $4.80(.789)$ \\
\hline & Students B & 10 & $4.50(1.179)$ & $4.60(.966)$ & $4.50(.972)$ \\
\hline & Experts & 9 & $4.78(1.093)$ & $4.44(1.130)$ & $4.50(.535)$ \\
\hline \multirow{3}{*}{$\begin{array}{l}\text { The knowledge } \\
\text { process related } \\
\text { variables }\end{array}$} & Students A & 10 & $4.10(1.101)$ & $3.50(1.269)$ & $3.60(1.075)$ \\
\hline & Students B & 10 & $4.20(1.135)$ & $4.00(.943)$ & $3.50(.850)$ \\
\hline & Experts & 9 & $3.89(1.453)$ & $3.89(1.167)$ & $4.00(1.000)$ \\
\hline \multirow[b]{3}{*}{ The interventions } & Students A & 10 & $3.90(1.101)$ & $3.70(1.494)$ & $4.50(.527)$ \\
\hline & Students B & 10 & $4.40(.843)$ & $4.70(.823)$ & $4.10(.994)$ \\
\hline & Experts & 9 & $4.33(1.118)$ & $3.89(1.054)$ & $4.33(1.000)$ \\
\hline \multirow[b]{3}{*}{ The events } & Students A & 10 & $4.40(1.350)$ & $4.60(1.265)$ & $4.50(1.179)$ \\
\hline & Students B & 10 & $4.20(1.135)$ & $4.30(1.059)$ & $4.20(1.033)$ \\
\hline & Experts & 9 & $4.89(.601)$ & $4.67(.866)$ & $4.56(1.014)$ \\
\hline \multirow{3}{*}{$\begin{array}{l}\text { The decay behavior of } \\
\text { the model }\end{array}$} & Students A & 10 & $4.50(.850)$ & $4.50(.850)$ & $4.70(.675)$ \\
\hline & Students B & 10 & $3.80(.919)$ & $4.90(.568)$ & $4.80(.919)$ \\
\hline & Experts & 9 & $4.63(1.408)$ & $4.50(1.414)$ & $4.38(1.302)$ \\
\hline \multirow{3}{*}{$\begin{array}{l}\text { The influence of } \\
\text { interventions }\end{array}$} & Students A & 10 & $3.40(1.506)$ & $4.10(.738)$ & $3.70(.823)$ \\
\hline & Students B & 10 & $3.40(1.350)$ & $4.50(.527)$ & $4.00(.816)$ \\
\hline & Experts & 9 & $4.44(.882)$ & $4.56(.726)$ & $3.67(1.118)$ \\
\hline \multirow{3}{*}{$\begin{array}{l}\text { The influence of } \\
\text { events }\end{array}$} & Students A & 10 & 4.00 (1.247) & $4.10(.738)$ & $3.40(.966)$ \\
\hline & Students B & 10 & $4.10(1.287)$ & $4.20(.789)$ & $4.40(.843)$ \\
\hline & Experts & 9 & $4.89(.928)$ & $4.56(.882)$ & $4.00(1.000)$ \\
\hline \multirow{3}{*}{$\begin{array}{l}\text { The behavior of the } \\
\text { model }\end{array}$} & Students A & 10 & $4.00(1.155)$ & $4.20(.789)$ & $4.10(.994)$ \\
\hline & Students B & 10 & $4.30(.483)$ & $4.10(.738)$ & $3.90(.738)$ \\
\hline & Experts & 9 & $4.89(.782)$ & $4.67(.707)$ & $4.11(1.054)$ \\
\hline \multirow[b]{3}{*}{ Aggregation } & Students A & 10 & 45.90 (5.65) & $45.70(5.16)$ & $46.50(5.79)$ \\
\hline & Students B & 10 & $45.30(5.46)$ & $47.10(5.11)$ & $49.10(5.45)$ \\
\hline & Experts & 9 & $51.00(6.35)$ & $47.57(7.72)$ & $47.88(7.68)$ \\
\hline
\end{tabular}

T-tests for independent samples showed that there is a significant difference between judgments of students and experts on only one validity proposition: the 
plausibility of knowledge processes. Experts judged the plausibility of knowledge processes significantly higher than students who performed above average $(\mathrm{t}(17)=-2.469$, $\mathrm{p}=.024)$ and students who performed below average $(\mathrm{t}(17)=-3.825, \mathrm{p}=.001)$. There were no additional significant differences found in judgments of students and experts.

Overall of 33 validity propositions, experts assessed 17 validity propositions more positively than did students. Only one validity proposition from the Plausibility category was assessed lower by experts than by students: the plausibility of the knowledge process related variables.

Experts gave higher scores for 24 validity propositions compared to students who performed above average and higher scores for 19 validity propositions compared to students who performed below average. This is an indication that students who performed better in the game thought about the model more critically.

In the plausibility category students who performed below average assessed 5 validity propositions lower and 5 validity propositions higher than students who performed above average. Students, who performed above average and below average, assessed one validity proposition in this category similarly. In the applicability category, students who performed below average assessed four validity propositions lower and 7 validity propositions higher then students who performed above average. In the credibility category, students who performed below average, assessed 5 validity propositions lower and 6 validity propositions higher then students who performed above average.

Based on the findings that students who performed below average did not assess most of the validity propositions lower than students who performed above average and that there were no more significant differences between judgments of this group of students and experts than between students who performed above average and experts, we conclude that the acceptance of the model and its components by students did not influence their playing behavior and, in this case, the internal educational validity of the model.

The difference that was found, in the mean judgments scores of students and experts concerns the plausibility of the knowledge processes and refers to the internal representational validity. Thus, the plausibility of knowledge processes could be considered as a 'validity bottleneck' which jeopardizes the model's educational validity. Nevertheless, given the fact that for 33 validity propositions there was a significant difference in judgments between students and experts for only one validity proposition, we cannot conclude that there is a difference in judgments on perceived representational validity (students' judgments) and on representational validity (experts' judgments). Therefore in the case of the model possessing a high level of functional fidelity, a high level of complexity and a moderate level of physical fidelity, perception of the model as a valid representation of reality does not influence the model's internal educational validity. These results confirm the findings of the first experimental study where we investigated relationships between perceived representational validity of the model and the model's educational validity and found no relationships between perceived representational validity of the model and its internal educational validity. 


\subsection{Discussion and conclusion}

This cross-study analysis reveals that the models which include the knowledge process related variables and possess higher and moderate levels of functional fidelity provide a higher level of internal educational validity than the model without the knowledge process related variables possessing a high level of physical fidelity and a low level of functional fidelity. At the same time, the internal educational validity can be increased if students get support on the unfamiliar variables and are assisted in dealing with the complexity of the model. That is demonstrated in this study by the availability of the supportive KM model, which guides students in the process of selecting "bottleneck" variables and making decisions. The model that has a higher level of physical fidelity did not prove to be educationally valid compared to models having lower levels of physical fidelity and higher levels of functional fidelity, but was assessed by students as having a higher internal and external representational validity.

Experts, in turn, assessed the model with a high level of functional fidelity, a high level of complexity and a moderate level of physical fidelity as being plausible, but less applicable and credible, while students assessed the same model as being less plausible. At the same time, for this model, judgments on perceived representational validity and representational validity do not differ much, which supports the idea that perceived representational validity does not strongly influence the educational validity of the model. As for the educational validity, a comparison of the game results between experts and students, who performed above average in the game showed that experts achieved the same good results as those students but at "lower costs" or more efficiently, which proves that the model possesses internal educational validity.

As KM Quest is an educative game, we should aim to achieve a higher level of educational validity rather than representational validity if faced with a choice between the two. The studies proved that the acceptance of the model as a valid representation of reality does not negatively influence the educational validity of the model. Overall, judgments about representational validity were above average, so it is probably not necessary to put more effort into increasing representational validity to improve educational validity as the gain in the latter in terms of the former will quite likely be negligible. In addition, from the results of the experimental studies on the KM Quest game simulation model, we conclude, that for achieving the highest educational validity, the model presented to the players, should possess a high level of fidelity (e.g. include all variables) and players should be supported in their decision making process by the supportive KM model, which decreases the complexity of the model. 



\section{Chapter 9}

\section{General Discussion}

This chapter presents a summary of the work and a reflection on research questions posed in the first chapters. The chapter ends with a discussion about possible research directions in future. 


\subsection{Summary}

The main goals of this research project were to develop a game simulation model which supports learning knowledge management knowledge and skills with the knowledge management game KM Quest and to investigate the validity of that model.

In a knowledge-driven economy knowledge is a competitive factor. Nowadays companies realize that there is a causal link between knowledge management activities in a company and its business performance. Yet there is little known about how exactly knowledge relates to the organizational outcomes and there are no models that contain such knowledge. The model, which was developed in this research, contributes to knowledge management theory by providing ideas about how knowledge is related to business outcomes. Moreover, the model supports learning about these phenomena, since players of the game could observe how knowledge management activities and dynamical changes in a company's knowledge household influence its organizational performance. Nevertheless, the model before getting in use should be validated from two perspectives: whether it reflects an actual phenomena and whether it supports learning about these phenomena. Therefore the main research questions in this research were:

- To what extent does the model reflect actual phenomena?

- Does the model support and provide learning about these phenomena?

- How can we increase validity of the model?

These research questions together with theoretical foundations to be considered during the research project are introduced in Chapter 1.

Chapter 2 is devoted to the model building process. It describes the development process: purpose of the model, requirements for the model and modeling assumptions which were made during the model building process. As a result of that process, the model for an abstract Product leadership company was developed. The model contains three knowledge domains: Marketing, Research \& Development, and Production. For these knowledge domains the following knowledge processes were identified: knowledge gaining, knowledge development, knowledge utilization, knowledge transfer, and knowledge retention. The company's performance, its knowledge domains and knowledge processes were formalized in a four layer model consisting of the following variables: knowledge process related variables, knowledge variables, business process related variables, and organizational effectiveness variables. The state of all variables changes over time (through the game periods) as a result of knowledge management activities (knowledge management interventions in the game), the game events, or decay behavior of knowledge process related variables. The decay function was introduced in the model to represent characteristics of knowledge such as ageing and volatility. Furthermore, a justification for how knowledge management interventions and game event were selected as well as their features and specifications are provided. Generally, the model works and relates knowledge to the business outcomes as follows: the knowledge management interventions, the game events, and decay functions influence the state of the knowledge process related variables. These influence the state of the knowledge variables and business process related variables, which in their turn determine the values of organizational effectiveness variables.

Chapter 3 focuses on the translation of the model into a computer program and model verification. Techniques which can be used for the model verification are 
introduced and discussed. The simulation modeling package is presented. The results of the model verification in the two prototype evaluations presented. It is concluded that the way the model is implemented in software, provides a guarantee that the theoretical aspects of the model are present in the actual simulation model.

Chapter 4 centers on validity issues. The need and purpose of the model's validation are explained. Two types of model validity are distinguished: educational validity and representational validity. Both types of validity are divided into two categories: internal and external representational validity and internal and external educational validity. Furthermore, the validity measurements for both types of validity are discussed.

We adopted the view that representational validity of the model can be tested only by subject matter experts, while learners playing the game can only report on their perception of representational validity (Feinstein \& Cannon, 2001; Francis \& Couture, 2003). Therefore we distinguish representational and perceived representational validity.

Since educational validity of the model can not be tested without extracting the model from the learning environment in which it is embedded, factors which could affect educational validity of the model are considered and the choice is made to investigate two factors. These factors are the model's fidelity, which is an internal factor from the model building point of view and mode of playing, which is an external factor from the model building point of view.

Furthermore, two types of knowledge, which players of the game should acquire during the game experience are presented. Strategic knowledge is defined as the ability of players to select and apply appropriate KM interventions in order to solve a KM problem. Conceptual knowledge consists of knowing KM bottlenecks and KM interventions, the relation between them and the propagation of the effects of interventions.

In line with the two factors which could influence model validity and the two types of knowledge which should be acquired when playing the game, the validity concepts used in this project are defined and research questions are made more specific. The model possesses internal representational validity if it provides a plausible representation of relationships between knowledge, knowledge management interventions, game evens and organizational performance. For possessing external representational validity the model should resemble a realistic organizational situation. The internal educational validity is defined as the ability of the model to support the acquisition of conceptual knowledge, while external educational validity is aimed at the acquisition of strategic knowledge. The research questions were refined as follows:

- To what extent does the model possess internal and external representational validity?

- Which model, in terms of possessing different levels of fidelity, provides higher internal and external educational validity and higher internal and external representational validity?

- Which mode of playing provides higher internal and external educational validity and higher internal and external representational validity?

- What kind of relationships exists between representational and educational validity of the model?

These research questions were answered in the next chapters describing the experimental studies. 
In the first experimental study we examined how different fidelity levels of the model affect educational and perceived representational validity of the model. This study is addressed in chapter 5. Different fidelity levels of the model were provided by closing access to the knowledge process related variables in the model for one group of players, while a second group of players had access to these variables. In this research we distinguish physical and functional fidelity (Hays \& Singer, 1989). The presence of knowledge process variables in the model decreases physical fidelity of the model, because these variables are not known and not used before in formalizing knowledge processes but increases in our view functional fidelity of the model since it clarifies how knowledge management activities (knowledge management interventions) influence knowledge processes in a company, its knowledge household and consequently its business outcomes. For teaching operational skills, a high level of physical fidelity of simulation models is important, while functional fidelity is more associated with teaching intuitive managerial skills. At the same time, presence of these variables increases the complexity of the model, because players of the game have to observe more variables. The following hypotheses were investigated:

- the model which includes knowledge process related variables (that is, the model possessing a higher level of functional fidelity and lower level of physical fidelity) provides a higher level of internal and external educational validity than the model which excludes these variables

- the perceived internal and external representational validity will be judged to be lower by players who could observe knowledge process related variables than by players who do not have access to these variables.

Fifty-two students from the University of Twente participated in this study. They were randomly assigned to one of the two conditions: a group of the players who could observe knowledge process related variables and a group of the players who could not observe knowledge process related variables. Internal educational validity was measured using game performance indicators and by achievements on a post test, assessing conceptual knowledge. External educational validity was measured using achievements on a post test assessing strategic knowledge. Perceived internal and external representational validity of the model was judged by participants of the game by means of the validity questionnaire.

Although the results of the game performance scores only indicated that students who could observe knowledge process related variables paid attention to these variables, the hypothesis that the model which includes knowledge process related variables provides a higher level of internal educational validity was only partially supported. The results of the post test measuring conceptual and strategic knowledge showed that students who could observe knowledge process related variables achieved higher scores on this test when compared to students who did not have access to these variables. Thus, the hypothesis that the model including knowledge process related variables provides a higher level of internal and external educational validity was confirmed. This suggests that knowledge process variables are important for teaching KM knowledge and skills.

As for perceived representational validity we did not find clear evidence that the presence of knowledge process related variables influences perceived internal and external representational validity of the model, although students who could not observe knowledge process related variables, judged the model and its components slightly more 
positive than students who had access to these variables. Our hypotheses that perceived internal and external representational validity of the model will be judged significantly higher by players who could not observe knowledge process related variables when compared to judgments of players who could observe these variables, is rejected.

Furthermore we investigated relationships between educational and representational validity from two perspectives: whether performance in the game affected validity judgments or whether the acceptance of the model as a valid representation of reality influenced educational validity. The correlation analysis reveals that players who achieved higher values of game performance indicators judge the model less positively. At the same time, results do not indicate that the presence of knowledge process related variables and consequently perception of the model as a valid representation of reality, influence educational validity of the model.

The second experimental study, which investigates the influence of the mode of playing on educational and representational validity of the model, is described in chapter 6. Since we did not find any evidence that different fidelity levels influence validity judgments in this study, the representation of the model was modified to possessing even less physical and functional fidelity when compared with the model representations in the first study. Players could not observe the business process related variables. The model contained knowledge process related variables, knowledge related variables, and only three, in practice well known, organizational effectiveness variables. Ninety-six students from the Radboud University Nijmegen participated in the experiment.

Thirty-six students played the game individually and sixty students were arranged in teams of two, which resulted in 30 teams. We hypothesized that a collaborative mode of playing will provide a higher level of internal and external educational validity and that mode of playing influences perceived representational validity. The measurements of educational and representational validity were the same as in the first study. Additionally, we included a pre-test assessing conceptual and strategic knowledge with the aim to find initial differences between subjects in two conditions.

Results of the post test assessing strategic knowledge revealed that a collaborative mode of playing provides a higher external educational validity. The game performance showed that teams outperform individuals only on one performance indicator, the hypothesis that a collaborative model of playing provides a higher internal educational validity was only partially supported. Post test scores on test items assessing conceptual knowledge did not differ significantly between the two conditions. Therefore, for a deeper analysis we compared scores on the pre and post test items assessing conceptual knowledge for subjects in the two conditions to find out whether subjects improved their conceptual knowledge at all. Results of this analysis reveal that only students who played collaboratively improved their conceptual knowledge significantly. This result supports the hypothesis that a collaborative mode of playing provides a higher internal educational validity. We did not find significant differences between the conditions in judgments on the validity propositions. The hypothesis that the mode of playing influences perceived internal and external representational validity is rejected. Overall, external representational validity was assessed by subjects in both conditions lower than internal representational validity. It can indicate that subjects accept the knowledge process related variables and knowledge variables as a game element, while such model features are seen as less fitting reality. 
Chapter 7 presents an exploratory study about the representational validity of the model. Representational validity of the model was evaluated with nine subject-matter experts in knowledge management with different backgrounds. External representational validity was assessed by the experts by means of a modeling questionnaire in which they had to indicate their judgments about several modeling decisions and statements which were made during the model design process. As for internal representational validity, experts evaluated specifications of ten knowledge management interventions, which are the part of model's internal logic and structure. Besides, after playing the game experts judged the external and internal representational validity of the model and its elements by answering the validity questionnaire, which we used in the previous experimental studies.

Results revealed that experts not always agree with our modeling decisions and specifications. Although some experts would like to have a more detailed model which has a lower level of abstraction and generalization, in general the majority of experts assessed the modeling assumptions and decisions positively. The degree of external representational validity is assessed by experts as being equal to eighty percent. The same result was found for internal representational validity. Assessment of internal and external representational validity by means of the validity questionnaire showed an even higher degree of validity. These results indicate that the model possesses internal and external representational validity to a large degree.

A combined analysis between the studies is provided in chapter 8. In the first section of this chapter we compared game performance, which is a particular case of internal educational validity, and judgments on internal and external representational validity between three groups of students: two groups of individual players from the first experimental study and the group of individual players from the second experiment. With respect to the different fidelity levels in the model which we varied by closing access to knowledge process related variables for one group of players and closing assess to business process related variables for the second group while third group could observe all variables in the model we look how these fidelity levels affect game performance and perceived representational validity. Complexity of the model was also included in the analysis. Results of the comparative analysis suggest that the model which includes knowledge process related variables, or the model with a higher level of functional fidelity provides a higher internal educational validity when compared with the model possessing a higher level of physical fidelity. At the same time the model with a higher level of physical fidelity (the model which does not include knowledge process related variables) provides a higher level of perceived internal and external representational validity. In the second part of this chapter we compared game performance of individual players from the first experiment with the game performance of experts in order to see whether the model possess internal educational validity as we expect that experts will outperform students. Additionally, we compared judgments of students and judgments of experts on representational validity in order to see how perceived representational validity differs from representational validity of the model. The game and model environment of students and experts were comparable, both groups could access all variables in the model. For a fair analysis we compared game performance of students who performed above average with the experts' game performance. Results revealed that experts achieved the same good results as students at lower costs, that is more efficiently, which confirms that the model possesses internal educational validity. Another finding is 
that judgments of perceived representational validity do not differ much from judgments on representational validity. This indicates that perceived representational validity does not influence educational validity of the model.

In the next sections, the work in this thesis is discussed with respect to the three main research questions posed in the first chapter and refined in chapter 4. Finally possible implications for future research are discussed.

\subsection{To what extent does the model reflect actual phenomena?}

With regard to the first research question, the issue is that the model should provide some ideas about how knowledge management activities and the knowledge household of a company influences its organizational performance and supports learning about this phenomenon.

Reflecting the model's purpose and the fact that is developed for an educational game, we have to be sure that players of the game deal with a valid representation of reality, otherwise they could arrive at wrong inferences about reality (Peters, Visser \& Heijne, 1998). From another point of view, we are dealing with an abstract phenomenon which was not formalized yet and other models do not exist. In this sense, the old management saying that we cannot manage what we cannot measure is in a slight contradiction with such intangible object of knowledge management as knowledge. However, when we recognize the importance of a concept, we can almost always find ways to measure it (Sterman, 2002). Thus, with the investigation of the game simulation model's validity we explore whether our attempt to formalize knowledge, knowledge processes, and relate them to business outcomes is successful.

The representational validity of the model was divided into two categories: internal representational validity and external representational validity. Internal representational validity is defined as the model's ability to provide a plausible representation of relationships between knowledge, knowledge management interventions, internal or external events and organizational performance in the game. The model possesses external representational validity when it resembles a realistic organizational situation.

From the many techniques available in the simulation research field to test representational validity of the model, we could apply in our investigation only informal methods, which rely heavily on human subjectivity. Such a limitation arose from the simple fact that there are no data available from the reality to test the model against. Thus, we invited subject matter experts in the knowledge management domain to judge our modeling assumptions and decisions, and evaluate the behavior of the model. We used two measurement instruments the modeling questionnaire and the validity questionnaire. Answering the modeling questionnaire experts judged our modeling assumptions concerning external representational validity and modeling decisions concerning internal representational validity. By answering the validity questionnaire experts judged both representational validity concepts.

The results revealed that the model possesses both external and internal representational validity to a degree of approximately 80 percent. We consider this to be a satisfactory result for a first attempt to formalize this abstract phenomenon. Although experts judged most of the modeling assumptions and decisions positively, there is room for improvement of the representational validity of the model. Concerning external 
representational validity, experts would like to see more detailed formalization of a company in terms of formalizing more knowledge domains and knowledge processes. Concerning internal representational validity experts would like the model to provide implementing scenarios in case things do not work as they are supposed to do after the implementation of knowledge management interventions. Additionally, specifications of some interventions jeopardize the model's internal representational validity. Changing of these specifications can increase this type of the model's validity.

Nevertheless there is a dilemma in formalizing this phenomenon, and this dilemma is related to the educational purpose of the model. We tried to generalize a model company as much as possible so that we do not have to teach knowledge management in one particular company, and let learners acquire knowledge that can be transferred to many other companies. In this sense, increasing representational validity of the model could hinder educational validity of the model as players of the game will not be able to acquire knowledge and skills on general level and transfer their knowledge and skills gained in the game to many companies instead of being limited to one specific organization. In fact this dilemma is in line with arguments put forward by Feinstein \& Cannon (2002) who argued that a game might be assessed positively as a learning tool, but could be less fitting real-world phenomena.

Concluding, it can be noted that given the positive results on the assessment of the model as possessing to high degree internal and external representational validity, it still does not make a model the absolutely 'right' model (if ever any exists), but it confirms that the model captures the essential features of knowledge management in organizations.

\subsection{Does the model support and provide learning about the phenomenon?}

Ability of the model to support and provide learning reflects its educational validity. In this study we distinguish two types of educational validity: internal educational validity and external educational validity. Internal educational validity is defined as the ability of the model to support the acquisition of conceptual knowledge in KM domain (e.g. players discern relationships of the modeled phenomenon and address them in the intended manner), while external representational validity reflects the ability of the model to support acquisition of strategic knowledge or, in other words, decision-making skills in the KM domain.

One of the starting points in our investigation is that we can not test the model's validity in a "pure" way. It is embedded in the learning environment and additional effects will always be present while investigating learning or knowledge gains. Therefore, we adopted a view that learning effectiveness of a simulation model and the game could be investigated by systematic variation of exactly one characteristic of a simulator (Größler, 2001). According to the taxonomy of Maier and Größler (2000) these characteristics that affect learning are located in three aspects of business simulations: underlying model, functionality, and human-computer interaction. In this study we choose two factors to investigate how educational validity of the model could be improved or in other words how these factors affect educational validity of the model. The first factor is the level of fidelity of the model, which belongs to the "underlying model' aspect of the simulation in the mentioned taxonomy. Additionally, we considered a factor which is not mentioned in this taxonomy and belongs to the instructional 
category: the mode of playing, collaborative or individual. Instructional categories always remain important in systems designed to teach specific knowledge and skills.

Thus, original research question was decomposed and refined as:

- Which model, in terms of possessing different levels of fidelity, provides higher internal and external educational validity?

- Which mode of playing provides higher internal and external educational validity?

In two experimental studies, described in chapter 5 and 6, an attempt was made to answer these questions.

As stated by several authors training of different knowledge and skills requires different levels of fidelity in simulations (Hays \& Singer, 1989; Maier \& Größler, 2000). Additionally, the level of fidelity and transfer of learning are connected (Alessi \& Trollip, 1991). Fidelity is a degree of realism that the simulation presents to the learner (Feinstein $\&$ Cannon, 2002). Adopting the view of Hays and Singer (1989), we distinguish physical and functional fidelity. By introducing knowledge and knowledge process related variables we decrease the level of realism in the game and therefore the physical fidelity of the model. Nevertheless, the assumption is that knowledge process related variables are needed to provide understanding how knowledge processes contribute to organizational outcomes. Thus these variables contribute to the functional fidelity of the model. The hypotheses were that the model including knowledge process related variables would lead to a higher level of internal and external educational validity than the model excluding these variables.

Results of this study revealed that the model possessing a higher level of functional fidelity (e.g. including knowledge process related variables) provides a higher level of internal and external educational validity. This indicates that knowledge process related variables are needed to provide understanding about how knowledge and knowledge management activities influence organizational performance.

As knowledge management problems are complex and ill-defined, we believe that a collaborative mode of playing provides for a better acquisition of conceptual and strategic knowledge because students in collaborative settings share their perspectives, ideas, and experiences. The hypotheses were made that a collaborative mode of playing leads to a higher degree of internal and external educational validity.

Results showed that there were no differences in test scores of subjects in the two conditions neither on items assessing conceptual knowledge nor on items assessing strategic knowledge. Players in the collaborative condition outperform individual students on one game performance indicator. The post test scores revealed that students who played collaboratively achieved a higher scores on the items assessing strategic knowledge, no difference was found on items assessing conceptual knowledge. These results were a bit ambiguous because strategic knowledge in the game is based on conceptual knowledge. Therefore, we conducted an additional analysis in order to see whether subjects improved their conceptual knowledge at all. Results indicated that only students who played collaboratively improved their conceptual knowledge. Thus, we concluded that a collaborative mode of playing proves to provide a higher level of internal and external educational validity.

Summarizing, in these experimental studies we investigated two factors which could affect educational validity of the model and consequently game educational 
validity. We found that knowledge process related variables, which do not exist in reality, are needed to provide a higher educational validity. Again, findings that functional fidelity is more important than physical fidelity in training of intangible managerial skills are confirmed. Besides, collaboration provides better learning of decision-making skills in the KM domain.

\subsection{How can we increase validity of the model?}

The main assumption behind this research question is that complexity of a game and acceptance of the model as a valid representation of reality influences playing behavior and learning results (Moizer, Lean, Towler \& Smith, 2006). We adopted a view that users and players of the game report on their perception of validity, while subject matter experts judge validity (Feistein \& Cannon, 2001). Thus, in two experimental studies we investigated how players of the game perceive internal and external educational validity in different conditions and whether there are relations between representational and educational validity of the model.

In the first experimental study, by introducing to players knowledge process related variables we decreased physical fidelity of the model and increased functional fidelity of the model as well as complexity of the model, because students have to observe more variables.

Students, who played the game with or without knowledge process related variables available, were asked to evaluate the model's internal and external representational validity by filling in the validity questionnaire. The hypotheses were made that students who could observe knowledge process related variables would judge the model's internal and external representational validity lower then the students who could not observe these variables, because these variables are not used in reality. Although results of the study revealed that students who could not observe knowledge process related variables judged the model's internal and external representational validity more positive then students who could observe these variables, no significant differences in judgment scores on validity propositions were found. Correlation analysis did not show that good or poor performance in the game influenced the validity judgments. Moreover, students who performed better in the game, judged the model less positively. This can indicate that these students think more deeply about the model. At the same time, we did not found evidence that acceptance of the model as valid representation of reality influences the model's educational validity. Thus the presence in the model of knowledge process related variables which increase the model's functional fidelity and complexity and decrease physical fidelity (or realism of the model), does not influence the model's perceived representational validity. In the next experimental study while looking how different mode of playing would affect validity judgments, we decreased both physical and functional fidelity of the model by closing access to business process related variables and making them unobservable.. In this experiment we did not find any evidence that players who played the game collaboratively judged the model differently from individual players. An explanation for not finding significant differences on perceived representational validity in these two experiments could be that the model possesses to some extent the representational validity or that players simply believe in 
simulation model as being true replication of reality (Meadows, 2001) since overall the model's perceived representational validity was assessed positively.

Given that players in all conditions perceive the mode's internal and external representational validity positively and no relationships were found between representational and educational validity, efforts to increase perceived representational validity of the model are probably not necessary. As for the educational validity of the model, for bringing it on higher levels, the model should include knowledge process related variables. Besides, a collaborative mode of playing showed better results concerning acquisition of strategic KM knowledge or decision-making skills in the KM domain.

\subsection{Directions for future research}

There are three promising research directions which can be followed in future. The first direction is linked to the first research question about the ability of the model to represent actual phenomena. In this study, while building the model, we used data available from the literature and relied on practical experience of project partners. The approach we have chosen has its limitations. First, we developed a theory how knowledge can be linked to organisational performance and then simulated reality. Afterwards, we asked subject matter experts to judge representational validity of the model based taking our modeling assumptions and decisions and the behavior of the model in the simulation as the starting point. This means that the model was never proven in reality. Longitudinal research in real companies could provide valuable data, not only about the ability of the model to represent reality but also about mistakes in the model structure and behavior, which could lead to the improvement of the model. At present, the model can be improved only by adjusting some knowledge management interventions. For example, changing the description of a problematic intervention concerning implementation of bonus system to improve employee effectiveness, which we discussed in chapter 7 , but not its specification, could easily increase the model's internal representational validity if 'bonus system' will be replaced with 'work facilities'. This is a simplified short-term solution, while the long term solution assumes research into actual behavior of organizations in practice.

The design of the studies and investigation of how different factors influence the model's educational validity lead us towards the second research direction in investigating the model's educational validity. It could be investigated whether the game provides learning with different fidelity levels in the model. For this, the design of the study should be changed towards a pre-post test design in order to see which conditions of the model support lead to more gain in conceptual and strategic KM knowledge. Another approach in this direction is to explore how different factors, for example, transparency of the KM interventions and propagation of their influences, could affect educational and perceived representational validity of the model.

The third direction of future research on the model's validity concerns two issues: relationships between perceived representational and educational validity and perceived representational validity of the model itself. In the present study we could not find evidence that perception of the model as a valid representation of reality affects playing behavior and learning results. We explained this from the fact that the model already 
possesses to some extent representational validity. A second explanation could be that people tend to believe that this model provides true causalities, as noted by Meadows (2001). It would be an ambition to purportedly incorporate in the model some errors which will cause implausible behavior of the model and to conduct controlled group experiments. Moreover, errors could be differentiated between serious and easy noticeable and simple and less noticeable errors. This experiment will allow a researcher to find out whether a direct relation exists between perceived representational validity of the model and its educational validity and whether judgments on representational validity will differ significantly.

Finally, a possible ultimate goal of knowledge management modeling as reported in this thesis is to make the science of knowledge management more precise and formal. Many texts on knowledge management are anecdotic and based on one shot case studies, or very much geared towards technical (that is computer based) solutions. Formal modeling and simulation are excellent tools to deepen our understanding of the complex relations that govern managing knowledge in an organization. 


\section{Nederlandse samenvatting}

Het onderzoek waarover deze dissertatie verslag legt was gericht op de ontwikkeling van een organisatie simulatie model voor het ondersteunen van het leren van kennismanagement en op de vraag wat de validiteit van dat model was.

De achtergrond is dat in een kennisgedreven economie kennis een belangrijke concurrentie factor is. Voor organisaties houdt dit in dat ze vertrouwd moeten zijn met de wijze waarop kennis management invloed heeft op de prestaties van de organisatie. Er is echter nog weinig bekend over deze relatie en modellen ontbreken geheel. Het model dat in dit onderzoek is ontwikkeld beoogt in deze lacune te voorzien en levert daarmee een bijdrage aan de theorie over kennismanagement. Daarnaast is het de bedoeling dat het model gebruikt wordt om kennismanagement te leren. Door met het model te spelen, kunnen lerenden onderzoeken hoe kennismanagement activiteiten en de daaruit voortvloeiende verandering in een organisatie invloed hebben op de prestaties van een organisatie. Het model en de bijbehorende leeromgeving zijn geïmplementeerd in het kennismanagement spel KMQuest.

Uiteraard staat of valt de waarde van het model met de vraag in hoeverre het gezien kan worden als een valide model. De validiteitsvraag wordt benaderd vanuit twee invalshoeken: is het model een afspiegeling van verschijnselen in de werkelijkheid en ondersteunt het model daadwerkelijk het leren over kennismanagement. Hieruit vloeien drie onderzoeksvragen voort:

- In welke mate weerspiegelt het model verschijnselen uit de werkelijkheid?

- Ondersteunt het model leren over deze verschijnselen

- Kunnen we de validiteit van het model verhogen?

Deze onderzoeksvragen worden geïntroduceerd en verder uitgewerkt in Hoofdstuk 1.

Hoofdstuk 2 gaat in het proces van het bouwen van het model. Aandacht wordt besteed aan: doel van het model, eisen die aan het model gesteld moeten worden en de veronderstellingen die aan het model ten grondslag liggen. Het model dat is ontwikkeld betreft een organisatie die als strategisch doel product leiderschap nastreeft. Voor zo'n organisatie zijn drie kennisdomeinen van doorslaggevend belang: Marketing, Onderzoek en ontwikkeling en Productie. Om de kwaliteit van deze kennisdomeinen op peil te houden of te verbeteren dient aandacht besteed te worden aan een reeks kennisprocessen: het extern verwerven van kennis, het intern ontwikkelen van kennis, het gebruik van kennis, de overdracht van kennis en tenslotte het behoud van kennis. De prestaties van de organisatie, de kennisdomeinen en de kennisprocessen zijn geformaliseerd in een vier lagen model dat bestaat uit de volgende variabelen: variabelen die de toestand van de kennisprocessen weergeven, variabelen die de toestand van de kennisdomeinen weergeven, variabelen voor bedrijfsprocessen en variabelen voor de prestaties van de organisatie. Al deze variabelen zijn aan verandering onderhevig als gevolg van kennismanagement activiteiten die de organisatie onderneemt, gebeurtenissen in en buiten de organisatie en de natuurlijke achteruitgang van kwaliteit wanneer een organisatie niets onderneemt. Deze laatste functie weerspiegelt processen zoals veroudering en veranderlijkheid van kennis. Daarnaast is een reeks interventies ontworpen en gemodelleerd. Deze interventies zijn acties die men kan ondernemen om in te grijpen in de kennisprocessen als gevolg waarvan de prestaties van de organisatie 
kunnen verbeteren. Samenvattend: management interventies, gebeurtenissen en natuurlijke achteruitgang veranderen de toestand van de variabelen die de kennisprocessen representeren. Als gevolg hiervan veranderen de variabelen voor de kennisdomeinen hetgeen propageert naar de bedrijfsprocessen en de prestaties van de organisatie. Dit dynamische is geïncorporeerd in het kennismanagement spel KMQuest.

In Hoofdstuk 3 wordt beschreven hoe dit model is omgezet in een computerprogramma. Daarbij is aandacht besteed aan de verificatie van het model, dat wil zeggen dat ervoor gezorgd moet worden dat er bij deze omzetting geen fouten optreden. Gebruik is gemaakt van een speciaal ontwikkeld simulatie constructie programma dat niet-programmeurs in staat stelt het model te programmeren. Het prototype is op twee verschillende manieren geëvalueerd en het resultaat is dat de manier waarop het model is omgezet in software garandeert dat de theoretische uitgangspunten aanwezig zijn in het werkende simulatie model.

Het validiteitsprobleem wordt verder uitgediept in Hoofdstuk 4. Twee typen validiteit komen aan de orde: educatieve validiteit en representatie validiteit. Beiden worden opgesplitst in een intern en een extern aspect. Daarnaast werd ervan uitgegaan dat representatie validiteit het beste kan worden beoordeeld door experts terwijl lerenden alleen een uitspraak kunnen doen over hoe ze deze validiteit percipiëren.

Educatieve validiteit kan niet getest worden zonder het model uit de leeromgeving waarin het zit ingebed te lichten, daardoor moeten factoren die de educatieve validiteit kunnen beïnvloeden worden geïdentificeerd. Twee factoren worden nader onderzocht: de manier waarop het model zichtbaar wordt gemaakt aan lerenden (deze factor is intern) en de manier waarop het KMQuest door lerenden gespeeld kan worden (deze factor is extern). Verder worden twee typen kennis die spelers moeten verwerven onderscheiden. Strategische kennis is kennis die te maken heeft met de bekwaamheid van de spelers om de passende interventies te kiezen waarmee kennisgerelateerde problemen in de organisatie kunnen worden aangepakt. Conceptuele kennis heeft betrekking op het op de hoogte zijn van kennis bottlenecks en interventies, de relatie daartussen het propageren van de effecten van interventies. Samenvattend: het model heeft interne representatie validiteit wanneer het een plausibele weerspiegeling is van relaties tussen kennis, kennismanagement interventies, gebeurtenissen in het spel en prestaties van de organisatie. Het heeft externe representatie validiteit wanneer het model een realistische afspiegeling is van een organisatie. Interne educatieve validiteit bestaat wanneer het model het verwerven van conceptuele kennis ondersteunt en externe educatieve validiteit wanneer hetzelfde geldt voor strategische kennis.

Op basis van deze onderscheidingen en definities zijn meer specifieke onderzoeksvragen geformuleerd:

- In welke mate bezit het model interne en externe representatie validiteit?

- Welke manier van het zichtbaar maken van het model leidt tot betere interne en externe educatieve validiteit?

- Welke manier van het spelen van KMQuest leidt tot een betere interne en externe educatieve validiteit en interne en externe representatieve validiteit?

- Is er een relatie tussen representatie validiteit en educatieve validiteit? 
Deze vragen zijn onderzocht via verschillende experimentele en niet experimentele studies die in de volgende hoofdstukken aan de orde komen.

De eerste experimentele studie in Hoofdstuk 5 onderzoekt hoe verschillende manieren waarop het model wordt zichtbaar gemaakt voor de spelers invloed heeft op educatieve validiteit en gepercipieerde representatie validiteit. Door de variabelen die de toestand van de kennisprocessen weergeven toegankelijk te maken voor een groep spelers en niet toegankelijk te maken voor een andere groep spelers werd de manier waarop het model zichtbaar wordt gemaakt gevarieerd. Hierbij wordt gebruik gemaakt van het onderscheid dat in de literatuur wordt gemaakt tussen fysieke en functionele waarheidsgetrouwheid. De zichtbaarheid van de kennisproces variabelen verlaagt de fysieke waarheidsgetrouwheid van het model omdat deze variabelen over het algemeen niet goed bekend zijn en nooit zijn gebruikt bij het formaliseren van kennisprocessen. De functionele waarheidsgetrouwheid daarentegen neemt toe omdat ze duidelijk maken hoe kennismanagement acties invloed uitoefenen op kennisprocessen in de kennishuishouding en daardoor op de prestaties van de organisatie. Voor het aanleren van operationele vaardigheden is een hoog nivo van fysieke waarheidsgetrouwheid vereist. Functionele waarheidsgetrouwheid is belangrijk voor het aanleren van meer intuitieve management deskundigheid. Daarnaast maakt de aanwezigheid van de kennisprocesvariabelen het model ook meer complex voor de spelers eenvoudigweg omdat er meer zaken zijn waarop gelet moet worden. De volgende hypothesen werden onderzocht:

- Het model waarbij de kennisprocesvariabelen zichtbaar zijn (het model met een grotere functionele waarheidsgetrouwheid en een lagere fysieke waarheidsgetrouwheid) leidt tot een hogere nivo van interne en externe educatieve validiteit dan een model waarin deze variabelen niet zichtbaar zijn voor de spelers.

- De gepercipieerd interne en externe representatie validiteit wordt lager beoordeeld door spelers die toegang hadden tot de kennisprocesvariabelen dan door spelers die daar geen toegang tot hadden.

Aan het onderzoek deden 52 studenten mee van de Universiteit Twente. Deze werden willekeurig toegewezen aan een van de twee condities: een conditie waarin men toegang had tot de kennisprocesvariabelen en een conditie waarin dit niet het geval was. Interne educatieve validiteit werd gemeten door te kijken op welk nivo de spelers de organisatorische prestatie indicatoren (o.a. winst) konden krijgen en scores op een posttest voor conceptuele kennis. Externe educatieve validiteit werd gemeten via een post-test vor strategische kennis. Gepercipieerde interne en externe representatie validiteit van het model werd gemeten met behulp van een validiteitsvragenlijst waarin verschillende aspecten van validiteit aan de orde werden gesteld.

Wat betreft de eerste hypothese kwam naar voren dat, op basis van de prestatie indicatoren, het model met de kennisprocesvariabelen slechts in beperkte mate leidde tot een hogere interne educatieve validiteit. Uit de resultaten van de post-test voor conceptuele en strategische kennis bleek dat studenten met toegang tot de kennisprocesvariabelen hoger scoorden dan studenten die geen toegang hadden. De 
hypothese werd dus bevestigd en dit toont aan dat kennisprocesvariabelen van belang zijn voor het onderwijzen van kennismanagement kennis en vaardigheden.

De hypothese betreffende gepercipieerde representatie validiteit kon niet worden bevestigd. Weliswaar beoordeelden studenten die geen toegang hadden tot de kennisprocesvariabelen het model en onderdelen daarvan enigszins positiever, maar de verschillen waren niet significant. Het wel of niet hebben van toegang tot de kennisprocesvariabelen heeft dus geen invloed op de gepercipeerde representatie validiteit.

Tenslotte werd de relatie tussen beide typen validiteit onderzocht. Dit gebeurde op twee manieren: beïnvloeden prestaties in het spel de gepercipieerde representatie validiteit en beïnvloed de gepercipieerd representatie validiteit de educatieve validiteit. Het bleek dat spelers die beter presteerden in het spel het model minder positief beoordeelden wat betreft representatie validiteit. Er werd geen relatie gevonden tussen toegang tot het model en gepercipieerd representatie validiteit en educatieve validiteit

Hoofdstuk 6 beschrijft de tweede experimentele studie. Deze onderzoekt de invloed van de manier waarop het spel gespeeld wordt op educatieve en representatie validiteit. Aangezien in de vorige studie geen relatie werd gevonden tussen de manier waarop het model zichtbaar is (fysieke en functionele waarheidsgetrouwheid) en de gepercipieerdede validiteit, werd de toegang tot het model op een andere manier beperkt door variabelen die bedrijfs processen representeren niet zichtbaar te maken. Dit leidt tot een lager nivo van fysieke en functionele waarheidsgetrouwheid van het model dan in de eerste experimentele studie. De manier van spelen werd gevarieerd door een groep deelnemers het spel alleen te laten spelen en een andere groep in een team van twee.

Aan het onderzoek deden 96 studenten mee van de Radboud Universiteit Nijmegen. Van deze speelden 36 studenten het spel alleen en de resterende 60 in teams van twee personen. De hypothese was dat teams een hoger nivo van interne en externe educatieve validiteit zullen bereiken en dat de manier van spelen invloed heeft op de gepercipieerde representatie validiteit. Educatieve en representatie validiteit werden op de dezelfde manier gemeten als in de eerste studie. Daarnaast werd een pre-test gebruikt voor het meten van conceptuele en strategische kennis om te kunnen bepalen of er verschillen waren in de beginsituatie van de condities.

Uit de post-test voor strategische kennis kwam naar voren dat teams hogere scores bereikten wat betreft externe educatieve validiteit dan van individuele spelers. Echter, teams doen het beter dan individuele spelers op slechts een prestatie indicator, dus de hypothese wordt slechts gedeeltelijk bevestigd. Tussen de twee condities warden geen verschillen gevonden op de post-test voor conceptuele kennis. Om beter inzicht te krijgen in deze resultaten werden de scores op de pre- en post test voor conceptuele kennis met elkaar vergeleken. Het bleek dat alleen spelers in een team hun conceptuele kennis significant wisten te verbeteren, hetgeen erop wijst dat het spelen in een team leidt tot een hogere interne educatieve validiteit.

Geen verschillen werden gevonden tussen de condities betreffende de gepercipieerde representatie validiteit. Wel werd over de hele linie de externe representatie valideit lager beoordeeld dan de interne representatie validiteit. Dit kan een indicatie zijn dat studenten weliswaar de zin inzien van de kennisprocesvariabelen en kennisvariabelen als onderdeel van het spel, maar niet zo sterk zien dat deze ook werkelijke verschijnselen weerspiegelen. 
Een meer exploratieve studie is het onderwerp van Hoofdstuk 7 waarbij de representatie validiteit van het model centraal stond. Deze werd beoordeeld door 9 experts op het gebied van kennismanagement met verschillende achtergronden. Externe representatie validiteit werd gemeten via een vragenlijst waar de experts konden aangeven in hoeverre zij het eens waren met verschillende beslissingen met betrekking tot het model en uitgangspunten tijdens het modelleerproces. Interne representatie validiteit werd gemeten door experts tien management interventies uit het model te laten beoordelen op verschillende aspecten. Verder speelden de experts het spel en beantwoordden de validiteitsvragenlijst die ook in de twee experimentele studies werden gebruikt.

Uit de resultaten kwam naar voren dat de experts het niet altijd eens waren met de modelleer beslissingen en specificaties van interventies. Over de hele linie werden deze echter positief beoordeeld, zij het dat sommige experts de voorkeur gaven aan een model met wat meer detail. Het oordeel over de externe representatie validiteit en de interne representatie validiteit kwam uit op $80 \%$, terwijl de oordelen in de vragenlijst nog positiever waren. Hieruit valt te concluderen dat naar het oordeel van de experts het model een hoge mate van interne en externe representatie validiteit bezit.

Door de manier waarop de verschillende studies zijn opgezet kan een vergelijking tussen deze gemaakt worden in Hoofdstuk 8. Eerst werden prestaties in het spel, een onderdeel van interne educatieve validiteit, en gepercipieerde interne en externe representatie validiteit vergeleken tussen drie groepen studenten: twee groepen individuele spelers uit de eerste experimentele studie en de individuele spelers uit de tweede experimentele studie. De waarheidsgetrouwheid van het model voor de drie groepen was als volgt: geen toegang tot de kennisprocesvariabelen voor de eerste groep, geen toegang tot variabelen voor bedrijfs processen voor de tweede groep, toegang tot alle variabelen voor de derde groep. Op deze manier kon gekeken worden of verschillen in waarheidsgetrouwheid effect hebben op prestaties in het spel en gepercipieerd representatie validiteit. Ook de complexiteit van het model werd meegenomen in de analyse. De resultaten geven een indicatie dat het model met toegang tot de kennisprocesvariabelen (het model met een hogere functionele waarheidsgetrouwheid) leidt tot een hogere interne educatieve validiteit dan een model met een hogere fysieke waarheidsgetrouwheid (het model zonder toegang tot de kennisprocesvariabelen). Tegelijkertijd leidt het model met een hogere fysieke waarheidsgetrouwheid (het model zonder toegang tot de kennisprocesvariabelen) tot een hoger nivo van interne en externe gepercipieerde representatie validiteit. Vervolgens werden prestaties in het spel van individuele spelers uit het eerste experiment vergeleken met die van de experts om te zien of het model intern educatieve validiteit bezit omdat we verwachten dat experts het beter zullen doen dan studenten. Tevens vergeleken we de oordelen over representatie validiteit van studenten en experts om te zien of de validiteitsoordelen van elkaar verschillen. Voor een faire vergelijking namen we de studenten die het beste presteerden. Naar voren kwam dat experts dezelfde goede resultaten boekten maar tegen lagere kosten, hetgeen aangeeft dat het model interne educatieve validiteit bezit. Validiteitsoordelen van studenten en experts lopen niet ver uiteen en zijn over het algemeen positief. Hieruit valt af te leiden dat gepercipieerde representatie validiteit geen invloed heeft op de educatieve validiteit van het model.

Tenslotte worden de drie onderzoeksvragen beantwoord en implicaties voor toekomstig onderzoek bediscussieerd. 



\section{References}

Alessi, S.M. (1988). Fidelity in the design of instructional simulations. Journal of Computer-based Instruction, 15(2), 40-47.

Alessi, S.M. \& Trollip, S.R. (1991). Computer-based Instruction: Methods and Development., 2nd ed., Englewood Cliffs, Prentice-Hall.

Anderson, R. \& McLean, R. (2000). Total Value Creation. CD-ROM available from CICA (Canadian Institute of Chartered Accountants).

Anjewierden, A., Shostak, I., \& De Hoog, R. (2002). KMsim: A Meta-modelling Approach and Environment for Creating Process-Oriented Knowledge management Simulations. In: A. Gomez Perez, V.R. Benjamins (eds). Proceedings of the 13th International Conference on Knowledge Engineering and Knowledge Management, EKAW 2002: Ontologies and the Semantic Web. Lecture Notes in Computer Science (LNAI) 2473, Berlin: Springer, 65-79.

Aversano, L., Bodhuin, T., Canfora, G., \& Tortorella, M. (2004). A Framework for Measuring Business Processes based on GQM. Proceedings of the Proceedings of the 37th Annual Hawaii International Conference on System Sciences (HICSS'04), January 05-08. Electronically available at: http://csdl2.computer.org/comp/proceedings/hicss/2004/2056/01/205610012a.pdf

Babbie, E. (1992). The practice of social research $\left(6^{\text {th }}\right.$ ed.). Belmont: Wadsworth.

Balci, O. (1998). Verification, validation, and testing. In: J. Banks, (Ed.), The Handbook of Simulation. Principles, Methodology, Advances, Applications, and Practice. New York: John Wiley \& Sons Inc., 335-393.

Banks, J. (1998). Principles of Simulation, In: J. Banks (Ed.): The Handbook of Simulation. Principles, Methodology, Advances, Applications, and Practice. New York: John Wiley \& Sons Inc., 3-30.

Berry, J. (2004). Tangible Strategies for Intangible Assets. New York: McGraw-Hill.

Boisot, M.H. (1999) Knowledge Assets: Securing Competitive Advantage in the Information Economy. Oxford: Oxford University Press.

Bontis, N. (2001). Assessing knowledge assets: a review of the models used to measure intellectual capital. International Journal of Management Reviews, 3, 1, 41-60.

Buckman, R.H. (2004). Building a Knowledge-Driven Organization. New York: McGraw-Hill.

Bunge, M. (1998). Philosophy of science: From Explanation to Justification (2002 ed.). New Brunswick (USA): Transaction Publishers.

Carlile, P.R., \& Christensen, C.M. (2005). The Cycles of Theory Building in Management Research. Working paper. Boston: Boston University and Harvard Business School. Electronically available at:

http://smgnet.bu.edu/smgnet/css/staff/pub/GetFile.cfm/WP2005-

03.pdf? wid $=1633 \&$ did $=508 \&$ Filename $=$ WP2005-03.pdf

Carson, J.S., II, (2002). Model Verification and Validation. In: E. Yucesan, C.H. Chen, J. L. Snowdon, and J. M. Charnes, (Eds.). Proceedings of the 34th Winter Simulation Conference: Exploring New Frontiers, San Diego, California, USA, December 811, 52-58. Electronically available at: http://www.informscs.org/wsc02papers/008.pdf 
Carvalho, G. F. (1991). Evaluating Computerized Business Simulators for objective learning validity. Simulation \& Gaming, 22, 3, 328-348.

Chwif, L., Barretto, M.R.P., \& Paul, R.J. (2000). On Simulation Model Complexity. In (Eds): J.A Joines, R.R. Barton, K.Kang, \& P.A. Fishwick. Proceedings of the 2000 Winter simulation Conference, Vol. 1, 449-455.

Christoph, N. (2006). The role of metacognitive skills in learning to solve problems. $\mathrm{PhD}$ Thesis, University of Amsterdam.

Christoph, N., Leemkuil, H.H., Ootes, S., Shostak, I., \& Monceaux, A. (2003). Final evaluation report on the use of the Final KITS learning environment prototype. EC project KITS (IST-1999-13078), KITS deliverable D14, Enschede: KITS consortium.

Couture, M. (2004). Realism in the design process and credibility of simulation-based virtual laboratory. Journal of Computer Assisted Learning, 20, 40-49.

Cronbach, L.J.(1990). Essentials of psychological testing (5th ed.). New York: Harper Collins.

Daft, R.L., \& Lengel, R.H. (1998). Fusion Leadership: Unlocking the Subtle Forces That Change People and Organizations. San Francisc, CA, Berett-Koehler Publishers.

Dalkir, K. (2005). Knowledge management in theory and practice. Oxford: Elsevier Inc.

Davenport, T.H. (1999). Knowledge Management and the Broader Firm: Strategy, Advantage, and Performance. In: J. Liebowitz (Ed.), The knowledge management handbook. Boca Raton: CRC Press, 2.1-2.11.

Davenport, T.H. \& Prusak, L. (1998). Working Knowledge: How Organizations Manage What They Know. Cambridge: Harvard Business School Press.

Drucker, P.F. (1998). Peter Drucker on the Profession of Management. Boston: Harvard Business School Publishing.

Dubin, R.. (1978). Theory Building (Revised ed.). New York: The Free Press.

Edvinsson, L. \& Malone, M.S. (1997) Intellectual Capital: Realizing your Company's True Value by Finding its Hidden Brainpower, NewYork: HarperCollins Publishers.

Faria, A.J. \& Wellington, W.J. (2005). Validating business gaming: Business game conformity with PIMS findings. Simulation \& Gaming, 36, 2, 259-273.

Feinstein, A.H. \& Cannon, H.M. (2002). Constructs of simulation evaluation. Simulation \&Gaming, 33, 4, 425-440.

Francis, A. \& Couture, M. (2003). Credibility of a simulation-based virtual laboratory: An exploratory study of learner judgements of verisimilitute. Journal of Interactive Learning Research, 14, 4, 439-464.

Gross, D. C. (1999). Report from the Fidelity Implementation Study Group. The Simulation Interoperability Standards Organization (SISO). Electronically available at: http://www.sisostds.org/index.php?tg=articles\&idx=More \&article=40\&topics $=18$

Größler, A. (2001). Musings about the effectiveness and evaluation of business simulators. Proceedings of the The19th International Conference of The System Dynamics Society July 23 - 27, 2001. Atlanta. USA. Paper electronically available at: http://www.systemdynamics.org/conferences/2001/papers/Groessler_1.pdf

Größler, A. (1998). Structural Transparency as an Element of Business Simulators. Paper presented at The 16th International Conference of The System Dynamics Society, 
July 20-23, Quebec, Canada. Electronically available at: http://www.systemdynamics.org/conferences/1998/PROCEED/00085.PDF

Größler, A., Maier, F.H., \& Milling, P.M. (2000). Enhancing Learning Capabilities by Providing Transparency in Business Simulators. Simulation \& Gaming, 31, 2, 257278.

Haldane, A. (2000). Requirements for the KITS learning environment. EC project KITS (IST-1999-13078), KITS deliverable D4, Enschede: KITS consortium. Electronically available at: http://kits.edte.utwente.nl/documents/D4.pdf

Hatzipanagos, S. (1995). Fidelity and complexity: Dimensions in the study of reality and abstraction in computer simulations. Proceedings of ICCE 95: International Conference on Computers in Education, Singapore, Charlottesville, VA: AACE, 105-110.

Hays, R.T., \& Singer, M.J. (1989). Simulation fidelity in training system design: Bridging the gap between reality and training. New York: Springer-Verlag.

Herskovitz, P. J. (1991). A theoretical framework for simulation validation: Popper's falsificationism. International Journal of Modelling and Simulation, 11, 56-58.

Herz, B. \& Merz, W. (1998). Experiental Learning and the Effectiveness of Economic Simulation Games. Simulation \& Gaming, 29,2, 238-250.

Holsapple, C. W. (2003). Knowledge and Its Attributes. In: C.W. Holsapple (Ed.), Handbook on Knowledge Management I. Knowledge Matters, Berlin: SpringerVerlag, 165-188.

Holsapple, C. W. \& Joshi, K.D. (2003). Knowledge Management Ontology. In: C.W. Holsapple (Ed.), Handbook on Knowledge Management I. Knowledge Matters, Berlin: Springer-Verlag, 89-124.

Hoog, R. de, Shostak, I., Purbojo, R., Anjewierden, A., Christoph, N.,Kruizinga, E. (2002). Final models and visualisation. EC project KITS (IST-1999-13078), KITS deliverable D12, Enschede: KITS consortium.

Hoog, R. de, Shostak, I., Purbojo, R., Van der Tang, F., Van Heijst, G., \& Kruizinga, E. (2001). Initial specifications of models and visualisation. EC project KITS (IST1999-13078), KITS deliverable D7, Enschede: KITS consortium.

Hoog, R. de,Van Heijst, G.,Van der Spek, R., Edwards, S.J., Mallis, R.,Van der Meij, B., \& Taylor, M.R. (1999). Investigating a Theoretical Framework for Knowledge Management: A Gaming Approach. In: J. Liebowitz (Ed.), The knowledge management handbook. Boca Raton: CRC Press, 10.1-10.18.

Irvine, S.R., Levary, R.R., \& McCoy, M.S. (1998). The Impact of Judgmental Biases on the Validation of Simulation Models. Simulation \& Gaming, 29, 2, 152-164.

Jaques, D. (1995). Games, simulations and case studies - a review. In D. Saunders (Ed.), The International Simulation and Gaming Yearbook. Volume 3. Games and simulations for business. London: Kogan Page.

Kaplan, R.S., \& Norton, D. P. (1996). The Balanced Scorecard:Translating strategy into action. Boston: Harvard Business School Press.

Kayes, A.B., Kayes, D.C., \& Kolb, D.A. (2005). Experiental learning in teams. Simulation \& Gaming, 36, 3, 330-354.

Kayworth, T. \& Leidner, D. (2003). Organizational Culture as a Knowledge Resource. In: C.W. Holsapple (Ed.), Handbook on Knowledge Management I. Knowledge Matters, Berlin: Springer-Verlag, 235-252. 
Klabbers, J.H.G. (2006). Artifact assessment versus theory testing. Simulation \& Gaming, $37,2,148-154$.

Klabbers, J.H.G. (1989). On the improvement of competence. In: J. H.G. Klabbers, W.J. Scheper, C. A. Th. Takkenberg \& D. Crookall (eds.), Simulation-gaming : on the improvement of competence in dealing with complexity, uncertainty and value conflicts : proceedings of the International Simulation and Gaming Association's 19th International Conference, Department of Gamma-Informatics, Utrecht University, The Netherlands, 16-19 August 1988, Oxford: Pergamon Press, 3-7.

Kocher, M.G., \& Sutter, M. (2007) Individual versus group behavior and the role of the decision making procedure in gift-exchange experiments. Empirica, 34, 63-88.

Kocher, M.G., \& Sutter, M. (2005) The decision maker matters: individual versus group behaviour in experimental beauty-contest games. The Economic Journal, 115 (January), 200-223.

Kolloffel, B.J. (2008). Getting the picture. The role of external representationa in simulation-based inquiry learning. $\mathrm{PhD}$ Thesis, Enschede: University of Twente.

Krathwohl, D.R. (1998). Methods of Educational \& Social Science Research. An integrated approach. USA: Addison-Wesley Educational Publishers.

Kriz, W.C., \& Hense, J.U. (2006). Theory-oriented evaluation for the design of and research in gaming and simulation. Simulation \& Gaming, 37, 2, 268-283.

Law, A.M., \& Kelton, W.D. (2000). Simulation modeling and analysis. $3^{\text {rd }}$ ed. New York: McGraw-Hill

Leemkuil, H. H. (2006). Is it all in the game? Learner support in an educational knowledge management simulation game. PhD Thesis, Enschede: University of Twente.

Leemkuil, H.H., Christoph, N., de Hoog, R., de Jong, A.J.M., Ootes, S., Purbojo, R., \& Shostak, I. (2002). Final specification of instructional envelope. EC project KITS (IST-1999-13078), KITS deliverable D13, Enschede: KITS consortium.

Leemkuil, H.H., de Jong, A.J.M., \& Ootes, S. (2000). Review of educational use of games and simulations. EC project KITS (IST-1999-13078), KITS deliverable D1, Enschede: University of Twente, KITS consortium. Electronically available at: http://kits.edte.utwente.nl/documents/D1.pdf

Lev, B. (2001). Intangibles: Management, Measurement, and Reporting. Washington: Brookings Institution Press.

Machuca, J.A.D. (2000). Transparent-box business simulators: An aid to manage the complexity of organizations. Simulation \& Gaming, 31, 2, 230-239.

Maier and Größler, 2000. What are we talking about? - A taxonomy of computer simulations to support learning. System Dynamics Review, 16, 2, 135-148.

Martin, E.L. \& Waag, W.L. (1978). Contributions of platform motion to simulator training effectiveness: Study I - Basic contact. Brooks Air Force Base, TX: Air Force Human Resources Laboratory.

Meadows, D.L. (2001). Tools for Understanding the Limits to Growth: Comparing a Simulation and a Game. Simulation \& Gaming, 32, 4, 522-536.

Mintzberg, H. (1983) Structure in Fives: Designing Effective organizations. Englewood Cliffs: Prentice-Hall. 
Moizer, J.,Lean, J., Towler, M. \& Smith, G. (2006). Modes of learning in the use of a computer-based business simulation game. International Journal of Learning Technology, 2, 1, 49-61.

Moya, L.J., McKenzie, F.D., \& Nguyen, Q. H. (2008). Visualization and rule validation in human-behavior representation. Simulation \& Gaming, 39, 1, 101-117.

Newgren, K.E., Stair, R.M. \& Kuehn, R.R. (1980). The Relationship Betweeen Group Size and the Learning Curve Effect in a Gaming Environment. In: D.C. Brenenstuhl and W.D Biggs (eds.), Experiential Learning Enters the 80's. Tempe: Arizona State University, 203-205.

Newman, B. (2003). Agents, Artifacts, and Transformations: The Foundations of Knowledge flows. In: C.W. Holsapple (Ed.), Handbook on Knowledge Management I. Knowledge Matters, Berlin: Springer-Verlag, 301-316.

Nonaka, I. (1998). The Knowledge-Creating Company. In: D. Neef, G. A. Siesfeld, \& J. Cefola (eds.), The Economic Impact of Knowledge. Woburn: ButterworthHeinemann, 175-188.

Nurmi, S., \& Lainema, T. (2003). Turbulence Ahead! Engaging Students with Authentic, Collaborative Problem Solving Activities. In: F. Percival, H. Godfrey, P. Laybourn, \& S. Murray (eds.), The International Simulation and Gaming Yearbook, Vol. 11, 205-211.

O'Dell, C. (1996). A current review of knowledge management best practice. Paper presented at the Conference on Knowledge Management and the Transfer of Best Practices. London: Business Intelligence.

O'Dell, C., Elliot, S., \& Hubert, C. (2003). Achieving Knowledge Management Outcomes. In: C.W. Holsapple (Ed.), Handbook on Knowledge Management 2. Knowledge Directions, Berlin: Springer-Verlag, 253-287.

O’Neil, H.F., Wainess, R. \& Baker, E.L. (2005). Classification of learning outcomes: evidence from the computer games literature. The Curriculum Journal, 16, 4, 455474.

Pedgen, C.D., Shannon, R.E., \& Sadowski, R.P. (1995). Introduction to simulation using $\operatorname{SIMAN}\left(2^{\text {nd }}\right.$ ed.). Hightstown: McGraw-Hill.

Peters, V., Vissers, G., \& Heijne, G. (1998). The Validity of Games. Simulation \& Gaming, 29, 1, 20-30.

Probst, G., Raub, S., \& Romhardt, K. (2000). Managing Knowledge:building Blocks for Success. Chichester: John Wiley \& Sons, Ltd.

Purbojo, R. (2005). To look or not to look. A study of visualisation supports in an educational business game. $\mathrm{PhD}$ Thesis, Enschede: University of Twente.

Randel, J.M., Morris, B.A., Wetzel, C.D., \& Whitehill, B.V. (1992). The effectiveness of games for educational purposes: a review of recent research. Simulation \& Gaming, 23, 3, 261-276.

Raser, J.C. (1969). Simulations and society: An exploration of scientific gaming. Boston: Allyn \& Bacon.

Reilly, R.t F. \& Schweihs, R. P. (1998). Valuing intangible assets. New York: McGrawHill.

Robinson, S. \& Pidd, M. (1998). Provider and customer expectations of successful simulation projects. Journal of the Operational Research Society. 49, 3, 200-209. 
Rodov, I. \& Leliaert, F. (2002). FiMIAM: financial method of intangible assets measurement. Journal of Intellectual Capital, 3, 3, 323-336.

Romme, A.G.L. (2004). Perceptions of the Value of Microworld Simulation: Research Note. Simulation \& Gaming, 35, 3, 427-436.

Sandole, D.J.D. (2003). Validating Simulation-Based Models on Conflict. Simulation \& Gaming, 34, 2, 249-280.

Sargent, R.G. (1998). Verification and Validation of Simulation Models. In: D. J. Medeiros, Edward F. Watson, John S. Carson, Mani S. Manivannan (eds.). Proceedings of the 30th conference on Winter simulation, December 13-16, Washington, D.C., United States, 121-130. Electronically available at: http://ieeexplore.ieee.org/iel4/5993/16056/00744907.pdf?isnumber=\&arnumber=74 4907

Sargent, R.G. (1991). Simulation Model Verification and Validation. In: B.L. Nelson, W.D. Kelton, G. M. Clark (eds.). Proceedings of the 23d conference on Winter simulation, 8-11 December 1991 , Phoenix, AZ, United States, 37-47.

Shavelson, R.J., Webb, N.M., Stasz, C., \& McArthur, D. (1988). Teaching mathematical problem solving: Insights from teachers and tutors. In R. Charles \& E. Silver (Eds.), Research agenda for mathematics education: The teaching and assessment of mathematical problem solving. Reston, VA: The National Council of Teachers of Mathematics, Inc. \& L. Erlbaum, 203-231.

Shostak, I., Anjewierden, A. \& De Hoog, R. (2002). Modelling and Simulating ProcessOriented Knowledge Management. Proceedings of the Third European Conference on Knowledge management. Trinity College, Dublin, Ireland, 24-25 September, 634-648.

Slavin, R.E. (1990). Cooperative learning: Theory, research, and practice. Englewood Cliffs, NJ: Prentice Hall.

Smith, M.K., Wood, W.B., Adams, W.K., Wieman, C., Knight, J.K., Guild, N., \& Su, T.T. (2009). Why Peer Discussion Improves Student Perfromance on In-Class Concept Questions, Science, 323, 122-124.

Stanislaw, H. (1986). Tests of computer simulation validity. What Do They Measure? Simulation \& Games, 17, 2,173-191.

Sterman, J.D. (2002). All models are wrong: reflections on becoming a systems scientist. System Dynamics Review, 18, 4, 501-531.

Sterman, J.D. (2000) Business Dynamics: Systems Thinking and Modeling for a Complex World, Irwin, McGraw-Hill.

Sterman, J.D. (1994) Learning In and About Complex Systems. System Dynamics Review, 10, 2-3, 291-330.

Sterman, J.D. (1988). A Skeptic's Guide to Computer Models, in L. Grant, Foresight and National Decisions. Lanham: University Press of America, 133-169. Revised and Reprinted in: Barney, G., Kreutzer W., and Garrett, M. (1991) Managing a Nation. Boulder: Westview Press, 209-230

Stone, D.N., \& Warsono, S. (2003). Does Accounting Account for Knowledge? In: C.W. Holsapple (Ed.), Handbook on Knowledge Management I. Knowledge Matters, Berlin: Springer-Verlag, 253-269.

Sveiby, K.E. (1997). The New Organizational Wealth: Managing and Measuring Knowledge-Based Assets, San Francisco: Berrett-Koehler Publishers Inc. 
Swaak, J. (1998). What-if; discovery simulations and assessment of intuitive knowledge. $\mathrm{PhD}$ Thesis, Enschede: University of Twente.

Szymankiewicz, J., McDonald, J., \& Turner, K. (1988). Solving business problems by simulations. London: McGraw-Hill.

Teasley, S.D. (1995). Communication and collaboration: The role of talk in children's peer interactions. Developmental Psychology, 31, 2, 207-220.

Thavikulwat, P. (2002). Model for Currency Exchange Rates and Its Validation. Simulation \& Gaming, 33, 1, 5-27.

Thomas, R., \& Hooper, E. (1991). Simulations: An opportunity we are missing. Journal of Research on Computing in Education, 23, 497-513.

Tiwana, A. (2002). The knowledge management toolkit: orchestrating IT, strategy, and knowledge platforms, $2^{\text {nd }}$ Ed., Upper Saddle River: Prentice Hall.

Tiwana, A. (2000). The Knowledge Management Toolkit: practical techniques for building a knowledge management system. Upper Saddle River: Prentice Hall.

Treacy, M. and Wiersema, F. (1995) The Discipline of Market Leaders. New York: Perseus Books.

Turban, E. \& Aronson, J.E. (2001). Decision Support Systems and Intelligent Systems, 6th Ed., Upper Saddle River: Prentice Hall.

Veerman, A.L., \& Veldhuis-Diermanse, A.E. (2001). Collaborative learning through computer-mediated communication in academic education. Paper presented at the EURO-CSLC, Maastricht, The Netherlands.

Washbush, J.\& Gosen, J. (2001). An exploration of Game-derived Learning in Total Enterprise Simulations. Simulation \& Gaming, 32, 3, 281-296.

Wellington, W. J.\& Faria, A. J. (1996), Team Cohesion, Player Attitude, And Performance Expectations In Simulation. Simulation \& Games,. 27, 1, 23-40.

Wiig, K. M. (1995) Knowledge Management Methods: Practical Approaches to Managing Knowledge, Arlington: Schema Press.

Wiig, K.M., Hoog de R., \& Spek van der R. (1997). Supporting knowledge management: A selection of methods and techniques. Expert Systems with Applications, 13, 1527.

Wolfe, J. (1997). The Effectiveness of Business Games in Strategic Management Course Work. Simulation \& Gaming, 28, 4, 360-376.

Wolfe, J. (1985). The Teaching Effectiveness of Games in Collegiate Business Courses. Simulation \& Gaming, 16, 3, 251-288.

Wolfe, J. \& Chacko, T.I. (1982). The effects of different team sizes on business game performance. Developments in Business Simulation \& Experiential Exercises, 9, 232-235.

Wolfe, J, \& Chanin, M. (1993). The integration of functional and strategic management skills in a business game learning environment. Simulation and Gaming, 24, 1, 3446.

Wolfe, J \& Gold, S. (2007). A study of business game stock price algorithms. Simulation \& Gaming, 38, 2, 153-167.

Wolfe, J., \& Jackson, R. (1989). An investigation of the need for algorithmic validity. Simulation \& Games, 20, 3, 272-291. 



\section{Appendices}

Appendix 1. Final set of variables accessible for the players

Appendix 2. Variables and interventions/events for the R\&D domain

Appendix 3. Example of the specification of interventions

Appendix 4. Example of the specification of events

Appendix 5. Post test item measuring conceptual knowledge

Appendix 6. Post test item measuring strategic knowledge

Appendix 7. The validity questionnaire

Appendix 8. The modelling questionnaire 
Appendix 1. Final set of variables accessible for the players

\begin{tabular}{|c|c|c|}
\hline Name of the variable & Symbol & Status \\
\hline \multicolumn{3}{|l|}{ Knowledge processes related variables } \\
\hline Speed of knowledge gaining in marketing & KGsM & State \\
\hline Speed of knowledge gaining in research & KGsR & State \\
\hline Speed of knowledge gaining in production & KGsP & State \\
\hline Effectiveness of knowledge gaining in marketing & KGeM & State \\
\hline Effectiveness of knowledge gaining in research & KGeR & State \\
\hline Effectiveness of knowledge gaining in production & KGeP & State \\
\hline Efficiency of knowledge gaining in marketing & KGefM & State \\
\hline Efficiency of knowledge gaining in R \& D & KGefR & State \\
\hline Efficiency of knowledge gaining in production & KGefP & State \\
\hline Speed of knowledge development in marketing & KDsM & State \\
\hline Speed of knowledge development in R \& D & KDsR & State \\
\hline Speed of knowledge development in production & KDsP & State \\
\hline Effectiveness of knowledge development in marketing & KDeM & State \\
\hline Effectiveness of knowledge development in R \& D & KDeR & State \\
\hline Effectiveness of knowledge development in production & KDeP & State \\
\hline Efficiency of knowledge development in marketing & KDefM & State \\
\hline Efficiency of knowledge development in R \& D & KDefR & State \\
\hline Efficiency of knowledge development in production & KDefP & State \\
\hline Speed of knowledge utilisation in marketing & KUsM & State \\
\hline Speed of knowledge utilisation in R \& D & KUsR & State \\
\hline Speed of knowledge utilisation in production & KUsP & State \\
\hline Effectiveness of knowledge utilisation in marketing & KUeM & State \\
\hline Effectiveness of knowledge utilisation in R \& D & KUeR & State \\
\hline Effectiveness of knowledge utilisation in production & KUeP & State \\
\hline Efficiency of knowledge utilisation in marketing & KUefM & State \\
\hline Efficiency of knowledge utilisation in R \& D & KUefR & State \\
\hline Efficiency of knowledge utilisation in production & KUefP & State \\
\hline Speed of knowledge transfer from R \& D & KTsR & State \\
\hline Effectiveness of knowledge transfer from R \& D & KTeR & State \\
\hline Speed of knowledge transfer from marketing & KTsM & State \\
\hline Effectiveness of knowledge transfer from marketing & KTeM & State \\
\hline Speed of knowledge transfer from production & KTsP & State \\
\hline Effectiveness of knowledge transfer from production & KTeP & State \\
\hline Efficiency of knowledge transfer from marketing & KTefM & State \\
\hline Efficiency of knowledge transfer from R \& D & KTefR & State \\
\hline Efficiency of knowledge transfer from production & KTefP & State \\
\hline Effectiveness of knowledge retention in marketing & KReM & State \\
\hline Effectiveness of knowledge retention in R \& D & KReR & State \\
\hline Effectiveness of knowledge retention in production & KReP & State \\
\hline \multicolumn{3}{|l|}{ Knowledge related variables } \\
\hline Average level of competence in marketing & $\mathrm{CM}$ & State \\
\hline Average level of competence in R\&D & CR & State \\
\hline Average level of competence in production & $\mathrm{CP}$ & State \\
\hline
\end{tabular}




\begin{tabular}{|c|c|c|}
\hline Business processes related variables & & \\
\hline Level of sales & SalesL & State \\
\hline Level of sales based on marketing & SalesL_M & State \\
\hline Level of sales based on product quality & SalesL_PQI & State \\
\hline Production level & ProdL & State \\
\hline Potential market share & MS_pot & State \\
\hline Average time for new product to market & ATM & State \\
\hline Average time to change for producing a new product & ATP & State \\
\hline Company product quality index & $\mathrm{PQI}$ & State \\
\hline Number of patents in pending & Pat_pen & State \\
\hline Number of new patents & Pat_new & State \\
\hline Number of products in development & Prod_dev & State \\
\hline Number of new products & Prod_new & State \\
\hline Number of company products & Prod & Output \\
\hline Number of company patents & Pat & Output \\
\hline Average job satisfaction index of employees & JSI & State \\
\hline \multicolumn{3}{|l|}{ Finance, personnel, and statistical indicators } \\
\hline Turnover & Turnover & Output \\
\hline Turnover year 1 & Turnover1 & Output \\
\hline Turnover year 2 & Turnover2 & Output \\
\hline Turnover year 3 & Turnover3 & Output \\
\hline Total operating expenses & TOE & Output \\
\hline Total operating expenses year 1 & TOE1 & Output \\
\hline Total operating expenses year 2 & TOE2 & Output \\
\hline Total operating expenses year 3 & TOE3 & Output \\
\hline Non-operating expenses & NOE & Output \\
\hline Non-operating expenses year 1 & NOE1 & Output \\
\hline Non-operating expenses year 2 & NOE2 & Output \\
\hline Non-operating expenses year 3 & NOE3 & Output \\
\hline R \& D expenses & Exsr & Output \\
\hline Training expenses & Exst & Output \\
\hline Other expenses & Exso & Output \\
\hline Profit year 1 & PY1 & Output \\
\hline Profit year 2 & PY2 & Output \\
\hline Profit year 3 & PY3 & Output \\
\hline Number of R \& D employees & EmpR & Output \\
\hline Number of marketing employees & EmpM & Output \\
\hline Number of other employees & EmpO & Output \\
\hline Total number of employees & Emp & Output \\
\hline \multicolumn{3}{|l|}{ Organisational effectiveness variables } \\
\hline Market share & MS & Output \\
\hline Profit & Profit & Output \\
\hline Customer satisfaction index & CSI & State \\
\hline
\end{tabular}


Appendix 2. Variables and interventions/events for the R\&D domain

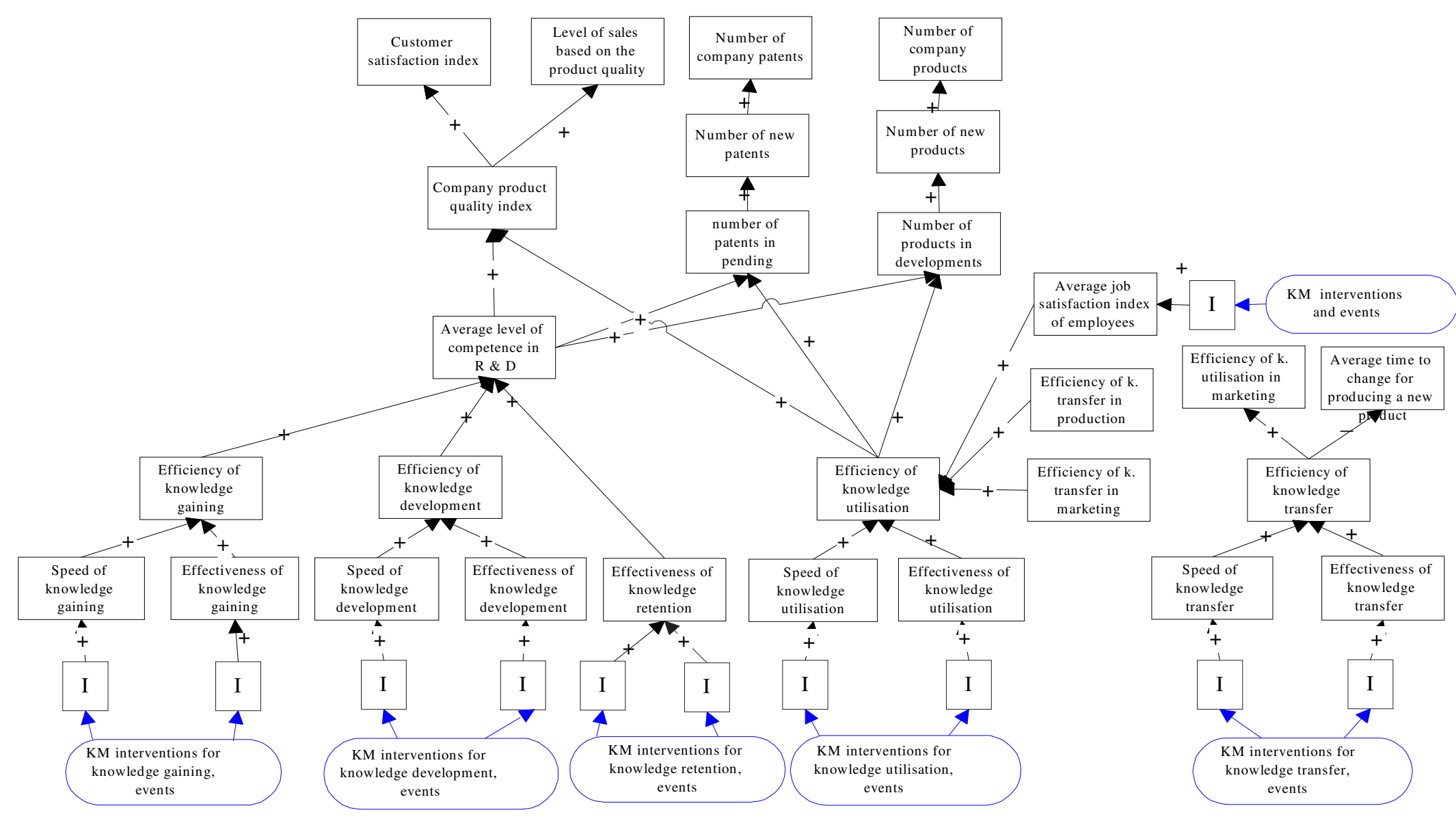


Appendix 3. Example of the specification of interventions

\begin{tabular}{|c|l|l|l|l|l|l|l|l|}
\hline Intervention & Variables & Delay & Initial & Next & Repeat & Cyclic & $\begin{array}{c}\text { Rep. } \\
\text { Eff }\end{array}$ & Reset \\
\hline I22 & Exst_A & 0 & 45000 & -45000 & 0 & no & 0 & no \\
\hline & KDAeM & 0 & 1.3 & -1.3 & 0 & no & 0 & no \\
\hline & KDAsM & 0 & 1.3 & -1.3 & 0 & no & 0 & no \\
\hline & KGAeM & 0 & 0.6 & -0.6 & 0 & no & 0 & no \\
\hline & KGAsM & 0 & 0.8 & -0.8 & 0 & no & 0 & no \\
\hline & KUAeM & 1 & 0.8 & -0.8 & 0 & no & 0 & no \\
\hline & KUAsM & 1 & 0.8 & -0.8 & 0 & no & 0 & no \\
\hline I38 & Exso_A & 1 & 10000 & -10000 & 0 & no & 0 & no \\
\hline & KDAeR & 1 & 0.9 & -0.7 & 0 & no & 0 & no \\
\hline & KDAsR & 1 & 0.9 & -0.7 & 0 & no & 0 & no \\
\hline & KRAeR & 0 & 0.8 & -0.4 & 0 & no & 0 & no \\
\hline & KTAeR & 1 & 0.8 & -0.5 & 0 & no & 0 & no \\
\hline & KTAsR & 1 & 0.8 & -0.5 & 0 & no & 0 & yes \\
\hline & KUAeR & 1 & 0.8 & -0.6 & 0 & no & 0 & no \\
\hline & KUAsR & 1 & 0.8 & -0.6 & 0 & no & 0 & no \\
\hline
\end{tabular}


Appendix 4. Example of the specification of events

\begin{tabular}{|l|c|c|}
\hline Event & $\begin{array}{c}\text { Enabling } \\
\text { conditions }\end{array}$ & Disabling conditions \\
\hline E17 & and $(\mathrm{JSI}<6, \mathrm{CP}<5.5)$ & intervention(I53, active, begin, -1$)$ \\
\hline E23 & none & none \\
\hline
\end{tabular}

\begin{tabular}{|c|l|l|l|l|l|l|l|}
\hline Events & Variables & Delay & Initial & Next & Repeat & Cyclic & Rep. Eff \\
\hline E23 & KTAeP & 0 & 0.4 & -0.4 & 0 & no & 0 \\
\hline & KTAeR & 0 & 0.4 & -0.4 & 0 & no & 0 \\
\hline & KTAsP & 0 & 0.5 & -0.5 & 0 & no & 0 \\
\hline & KTAsR & 0 & 0.5 & -0.5 & 0 & no & 0 \\
\hline
\end{tabular}




\section{Appendix 5. Post test item measuring conceptual knowledge}

\section{Part 1}

In this assignment, you have to introduce to the general management of Coltec some KM interventions. They can approve your KM initiatives only if they clearly foresee the outcomes of the interventions. Please specify for each intervention how and which knowledge domains and knowledge processes can influence Coltec. You can specify the influence by writing "=" or "_" in the direct influence field or in the delayed influence field for each knowledge domain and process. If there is no influence then please leave the certain field blank.

' + ' in the direct influence field means positive direct influence

'- ' in the direct influence field means negative direct influence

' + ' in the delayed influence field means positive delayed influence

'-' in the delayed influence field means negative delayed influence.

1. Description of intervention

Hire a high-class expert with new marketing knowledge on a temporary basis

- Indicate which knowledge domains and knowledge processes the intervention influences

\begin{tabular}{|l|l|l|l|}
\hline Domains & Processes & Direct influence & Delayed influence \\
\hline \multirow{5}{*}{ Marketing } & Gaining & & \\
\hline & Development & & \\
\hline & Utilisation & & \\
\hline & Transfer & & \\
\hline & Retention & & \\
\hline \multirow{5}{*}{ R\&D } & & & \\
& Gaining & & \\
\hline & Development & & \\
\hline & Utilisation & & \\
\hline & Transfer & & \\
\hline & Retention & & \\
\hline \multirow{5}{*}{ Production } & & & \\
\hline & Gaining & & \\
\hline & Development & & \\
\hline & Utilisation & & \\
\hline & Transfer & & \\
\hline & Retention & & \\
\hline
\end{tabular}




\section{Appendix 6. Post test item measuring strategic knowledge}

In this assignment, you as a knowledge manager of Coltec have to analyse five events that happened outside or inside of Coltec and take knowledge management initiatives.

\section{Description of Event}

Until now there has been a fairly stable preference of customers of coatings for a limited set

of colours. Recent market research has shown that this preference will be less and less stable

in the future leading to short term swings in demand, depending on the current colours en

vogue.

- Indicate what kind of event this is:

口 An internal problem/threat but not KM related

- An internal problem/threat that calls for KM actions

口 An external problem/threat but not KM related

- An external problem/threat that calls for KM actions

口 An internal opportunity but not KM related

- An internal opportunity that calls for KM actions

- An external opportunity but not KM related

- An external opportunity that calls for KM actions

- Indicate what effect can the event have on knowledge processes, on specific types of knowledge and/or business indicators.

The general management of Coltec expects from you as a knowledge manager, an advice about which actions to take to react upon the KM related problem, threat or opportunity that the company is facing (in KM Quest ${ }^{\mathrm{TM}}$ we do not talk about actions but about interventions that should be implemented).

- Based on the information you have so far, which (set of) knowledge management interventions would you propose to the management of Coltec and what are their goals? 


\section{Appendix 7. The validity questionnaire}

Before answering the following questions, we would like you to read carefully the following explanations. It will provide you with the modelling assumptions that are built into the conceptual model and explain the model structure.

Modelling assumptions:

1. Knowledge is a quantifiable entity. We measure it on the scale from 0 (lowest value - no specific knowledge available in a company) to 10 (highest value).

2. Performance of any business unit depends on the level of knowledge and the level of knowledge usage or utilisation. (Consequently, the business results also depend on the level of knowledge.) The ideal situation for a company is to have highly knowledgeable employees and an effective application of knowledge.

3. Knowledge naturally depreciates due to ageing and volatility. If there is no increase and renewal of knowledge in a company, the performance declines over time.

4. Changes from outside or inside a company influence the knowledge household of the company.

Model structure:

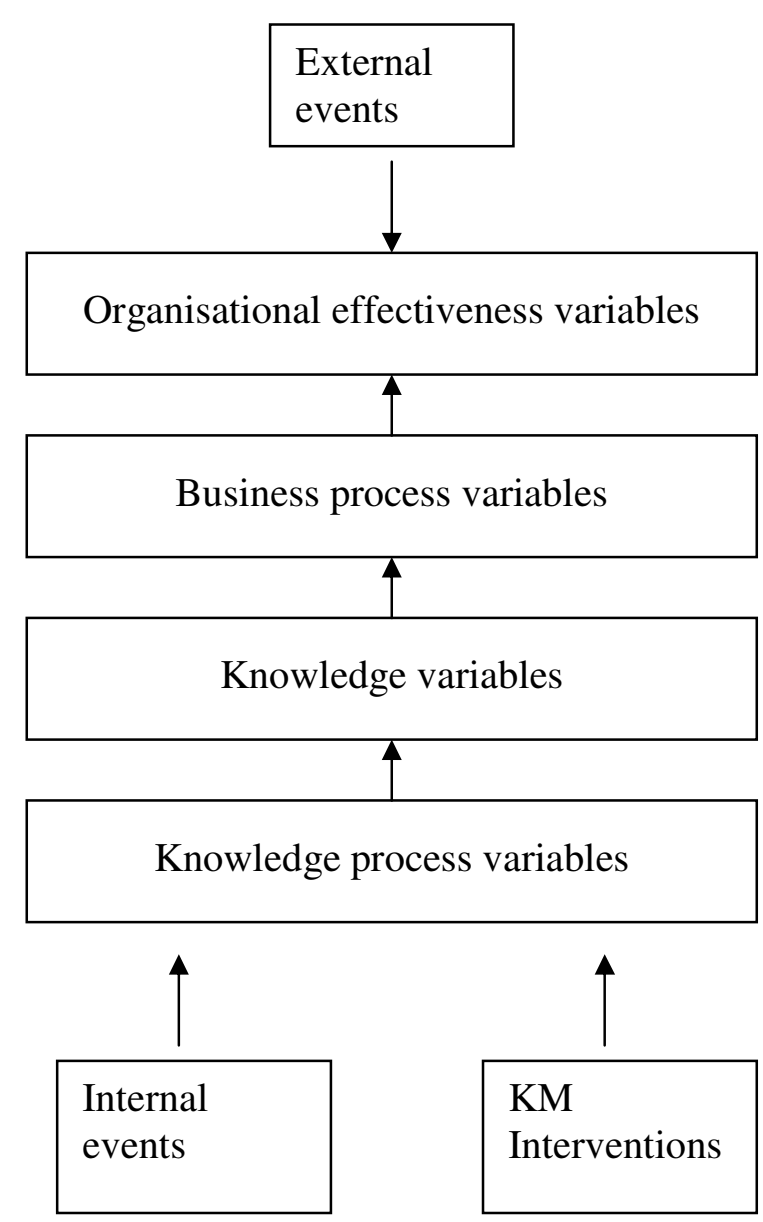


1. Organisational effectiveness variables. These variables represent the relation between the organisation and its environment. They reflect the competitive characteristics of the company and are introduced by variables like profit or level of sales.

2. Business process variables. These variables reflect the quality of internal processes - the ways things are done and how well they are done inside the company. An example is the production level.

3. Knowledge variables. They represent the levels of knowledge in relevant knowledge domains (e.g. marketing or production) and the quality of applying knowledge for each domain. An example is the level of competence.

4. Knowledge process variables. Reflect the properties of processes involving knowledge in the organisation. An example is the efficiency of knowledge transfer.

Simply stated, any event that happens outside or inside the company and any interventions taken inside the company can have an influence on the knowledge processes. These influence the "state" of knowledge in the organisation, which influence business processes and determine their quality. Finally, the business processes contribute and generate the values of the key organisational variables like profit and market share.

Now please fill in the following questionnaire.

$\begin{array}{cccccc}\begin{array}{c}\text { Strongly } \\ \text { Disagree }\end{array} & \text { Disagree } & \begin{array}{c}\text { Slightly } \\ \text { Disagree }\end{array} & \begin{array}{c}\text { Slightly } \\ \text { Agree }\end{array} & \text { Agree } & \begin{array}{c}\text { Strongly } \\ \text { Agree }\end{array} \\ 1 & 2 & 3 & 4 & 5 & 6 \\ 1 & 2 & 3 & 4 & 5 & 6\end{array}$

3. The explained conceptual model is realistic.

4. The defined knowledge domains are adequate for learning KM in the game.

5. The defined knowledge domains can be used

6. The defined knowledge domains exist in 
8. The defined knowledge processes can be

9. The defined knowledge processes exist in product leadership companies in reality.

10. The business process variables are adequate for monitoring performance of the company (Coltec) in the game.

11. The business process variables can be used performance.

12. The business process variables are realistic to be used by product leadership companies for monitoring their performance.

13. The knowledge process related variables are adequate for monitoring performance of the company (Coltec) in the game.

14. The knowledge process related variables can be used by other companies for monitoring their performance.

15. The knowledge process related variables are realistic to be used by product leadership companies for monitoring their performance.

16. The defined interventions are adequate for solving the KM problems in the game.

17. The defined interventions can be applied by other companies for solving KM problems.

18. The defined interventions are realistic to be applied by product leadership companies to solve KM problems.

19. The given events are adequate representations of KM problems in the game.

20. The given events can be experienced by other companies. 
21. The given events represent practical KM problems experienced by product leadership companies.

22. The decay behaviour of the knowledge and knowledge processes in the game is adequate for learning $\mathrm{KM}$.

23. The decay behaviour of the knowledge and knowledge processes can occur in other companies.

24. The decay behaviour of the knowledge and knowledge processes occurs in product leadership companies in reality.

25. The way the interventions influence company performance in the game is adequate for learning the impact of KM interventions.

26. The way the interventions influence company performance in the game can be the same for other companies.

27. The way the interventions influence company performance in the game is a realistic representation of interventions and their influences in product leadership companies.

28. The way the events influence company performance in the game is adequate for learning about impacts of events in the game.

29. The way the events influence company performance in the game can be in principle the same for other companies.

30. The way the events influence company performance in the game is a realistic representation of events influences on product leadership companies.

31. The business model exhibited adequate behaviour for learning knowledge management during the game.

32. The behaviour of the business model can represent behaviour of other companies.

33. The behaviour of the business model reflects actual behaviour of the product leadership companies. 


\section{Appendix 8. The modeling questionnaire}

To make a business model, which describes / represent a company for the purpose of learning knowledge management in a game context we have made several modeling assumptions.

We would like to know your opinion about these assumptions and their applicability for modeling a KM game and reality.

Please read explanations with the followings statements and give your opinion.

\section{Part one. Selection of knowledge domains and knowledge processes}

To model a company we had to start with defining a type of company and its knowledge household. For the knowledge management game we did not want to model a specific company, but make a model which:

1) Provides the learning of knowledge management by showing importance of knowledge and knowledge management activities;

2) Is understandable and applicable to certain extent to many companies.

As a starting point, we took classification made by Treacy and Wiersema (1995). They distinguished three main types of the companies:

1. Operational excellence organisations that compete on products that are cheaper.

2. Customer intimacy organisations that compete on products that are tailor-made.

3. Product leadership companies that compete on products that are innovative.

For the game we decided to model a Product leadership company and specified the following knowledge areas or domains that are crucial for this type of a company: $R \& D$, Marketing and Sales, and Production.

\section{Statement 1}

These knowledge domains are sufficient to represent knowledge household of a company and are needed to be modeled to represent a fictitious product leadership company in the game context.

$\begin{array}{cccccc}\text { Strongly } & \text { Disagree } & \begin{array}{c}\text { Slightly } \\ \text { Disagree }\end{array} & \begin{array}{l}\text { Slightly } \\ \text { Agree }\end{array} & \text { Agree } & \begin{array}{c}\text { Strongly } \\ \text { Agree }\end{array} \\ ( & ( & ( & ( & ( & (\end{array}$

If you do not agree please specify knowledge domains which are missing in your opinion. 
To show dynamics of knowledge in the company we had to:

1) Prioritize the importance of the knowledge areas for the company - to show the importance of different knowledge areas for the competitive position of the product leadership company on the market

2) Model different knowledge processes - processes that change the knowledge household and knowledge infrastructure of the company.

We are aware, that for a company all knowledge, which is used in this company, is important. At the same time, different knowledge brings the competitive advantages or disadvantages. Therefore, the importance of different knowledge areas for a company can be prioritized in accordance to the firm's strategic orientation.

\section{Statement 2}

Speaking about identified knowledge domains for a product leadership company, we define that the most crucial area is $\mathrm{R} \& \mathrm{D}$ since the company competes on the development of the innovative products. Marketing and sales knowledge has an average priority since company has to market those products skillfully and know market requirements. The production knowledge domain has the lowest priority.

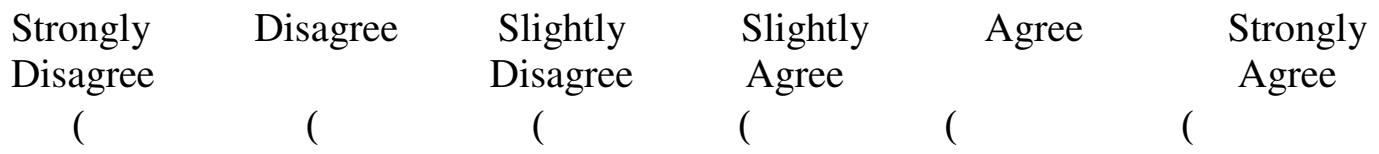

If you do not agree with our prioritization, please give your prioritization of knowledge domains for a product leadership company.

1. Knowledge domain with the highest importance.........

2. knowledge domain with the average importance

3. Knowledge domain with the lowest importance.

For modeling knowledge processes which change the knowledge infrastructure of the company we selected the following processes:

- Knowledge gaining. The process of getting new knowledge that is relevant for the company from the outside world.

- Knowledge development. The process of growing individual and organisational knowledge inside a company.

- Knowledge utilisation. The process of applying knowledge to the job.

- Knowledge transfer. The process of passing on knowledge to other business process areas, or making it available for further use in other business process areas.

- Knowledge retention. The process of preserving knowledge that is relevant for the company. 


\section{Statement 3}

These knowledge processes are needed to represent knowledge processes in a company and are sufficient to model a fictitious product leadership company in the game context.

$\begin{array}{cccccc}\begin{array}{c}\text { Strongly } \\ \text { Disagree }\end{array} & \text { Disagree } & \begin{array}{c}\text { Slightly } \\ \text { Disagree }\end{array} & \begin{array}{l}\text { Slightly } \\ \text { Agree }\end{array} & \text { Agree } & \begin{array}{c}\text { Strongly } \\ \text { Agree }\end{array} \\ ( & ( & ( & ( & ( & (\end{array}$

If you do not agree, please specify knowledge processes which are missing in your opinion.

\section{Model relationships}

Having those knowledge domains and knowledge processes we had to show their contribution to the business outcomes of the company.

\section{Statement 4}

The business outcomes of any unit of a company depend on the level of knowledge (competence) and efficiency of knowledge usage in this unit, if there are no additional influences from outside the unit.

This statement is valid to be modeled in the game context to provide understanding how knowledge contributes to the unit or firm's outcomes

$\begin{array}{cccccc}\begin{array}{c}\text { Strongly } \\ \text { Disagree }\end{array} & \text { Disagree } & \begin{array}{c}\text { Slightly } \\ \text { Disagree }\end{array} & \begin{array}{l}\text { Slightly } \\ \text { Agree }\end{array} & \text { Agree } & \begin{array}{c}\text { Strongly } \\ \text { Agree }\end{array} \\ ( & ( & ( & ( & ( & (\end{array}$

If you do not agree, please explain how knowledge domains and knowledge processes can influence business outcomes 


\section{Statement 5 (with picture)}

1.Level of knowledge (level of competence) in the business unit depends on the efficiency of knowledge gaining, knowledge retention and knowledge development. Depends means how well and effective those processes are organized in the company.

2.Efficiency of knowledge utilization depends on the knowledge infrastructure of the company and the level of employee satisfaction

3.Changes outside or inside the company influence the knowledge infrastructure, knowledge areas and knowledge processes

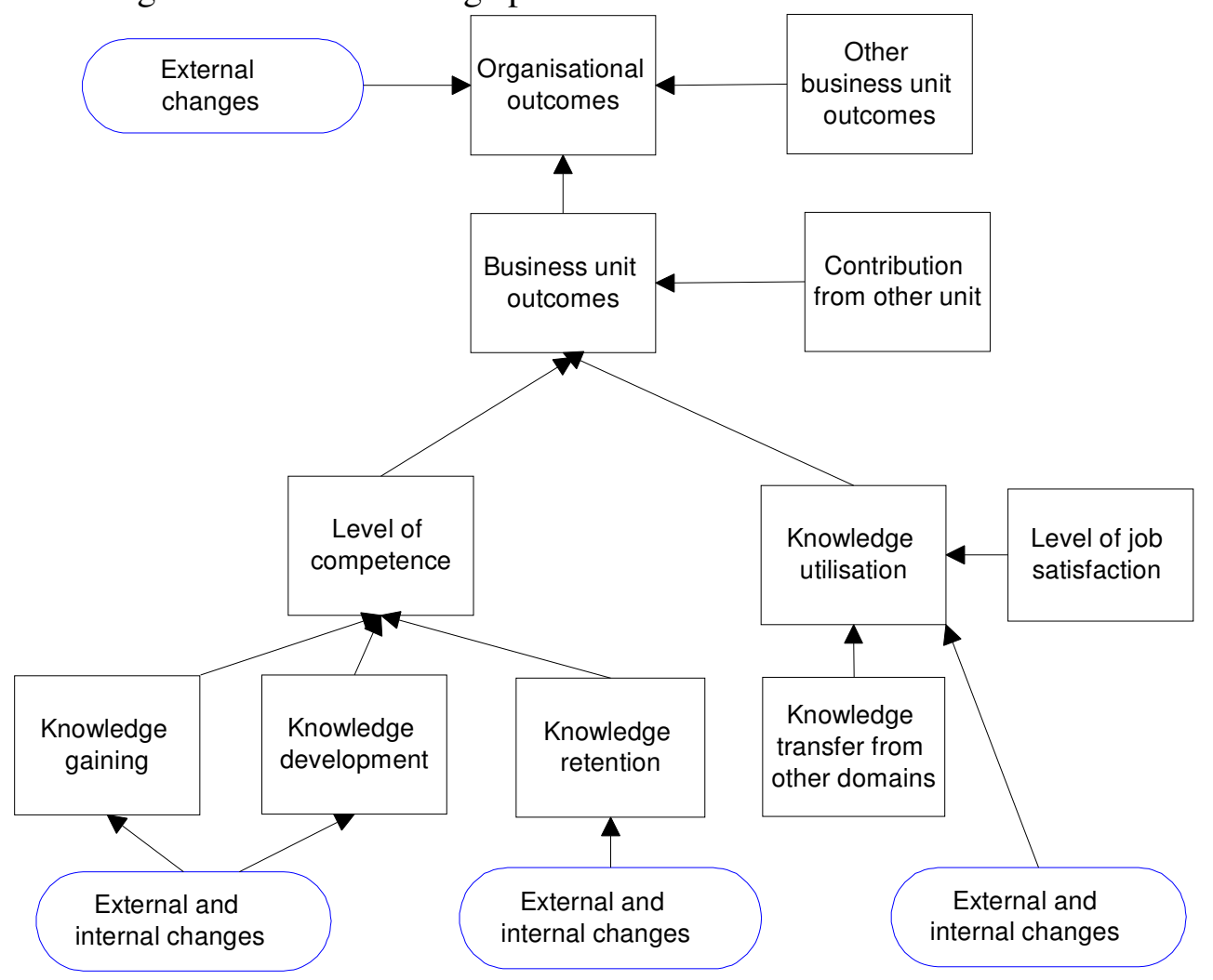

This statement is valid to be modeled in the game context to provide understanding how knowledge and knowledge processes contribute to the unit or firm's outcomes

$\begin{array}{ccclcc}\begin{array}{c}\text { Strongly } \\ \text { Disagree }\end{array} & \text { Disagree } & \begin{array}{c}\text { Slightly } \\ \text { Disagree }\end{array} & \begin{array}{l}\text { Slightly } \\ \text { Agree }\end{array} & \text { Agree } & \begin{array}{c}\text { Strongly } \\ \text { Agree }\end{array} \\ ( & ( & ( & ( & ( & (\end{array}$


If you do not agree with our view on the model relationships, please specify what is missing in your opinion.

\section{Part two. Influence of interventions and events}

In the game different events can happen outside or inside the company which influence the knowledge infrastructure of the company and/or its knowledge areas and processes. At the same time the player can also influence the knowledge infrastructure of the company, its knowledge areas and processes by implementing knowledge management activities - knowledge management interventions.

These events or interventions can have different influences on:

- one or more knowledge domains,

- one or more knowledge processes,

- market share and the job satisfaction level.

In addition these influences can be:

- immediate or delayed

- single or multiple influence

- negative or positive influence

- different in persistency (some influences persist over a longer period then others).

Please read carefully our clarifications about the model below:

- Knowledge transfer is transfer of knowledge between knowledge domains. It happens if there are communication and information flow processes between knowledge domains.

- Transfer or sharing of knowledge within a knowledge domain is a knowledge development process. Knowledge development processes also include training of personnel.

- Gaining of knowledge happens if a company hires external "services" provided by other companies (including partners) or people, or supply its personnel with additional information sources.

- Knowledge utilisation is the application of knowledge to the performance of daily tasks and can depend on employee motivation, attitude and satisfaction; work facilities and elements of the knowledge infrastructure (IT solutions).

- Knowledge retention means that knowledge and knowledge agents stay in the company and explicit knowledge is codified safeguarded against loss. 
Below we will give descriptions of influences of several interventions, based on how they are specified in the current business model. We ask you to "translate" these descriptions of influences in formal specifications if you are agree with them .If you don't agree, we ask you to enter you own formal specifications of the influences as you see fit.

Specification of influences for knowledge management interventions

Please specify the influences of interventions with the "+" symbol for a positive influence or the "-" symbol for a negative influence in the related knowledge domain-knowledge process field(s), either as an immediate or a delayed one. Please specify the duration of influence with symbol "x" in either the "short-term" or "long-term column".

Here is an example of the description of the intervention and its formal specification.

\section{Intervention - example}

Hire a high-class expert with new research knowledge permanently

\section{Description of intervention}

This intervention provides knowledge gaining in research and development domain since a person with new knowledge is coming to work in the R\&D department. Additionally this intervention improves knowledge utilisation in $R \& D$ department as this person applies his/her knowledge to work. The intervention has an immediate effect, which persist over a long period.

\section{Formal specification}

\begin{tabular}{|l|l|l|l|l|l|}
\hline Domains & Processes & $\begin{array}{l}\text { Immediate } \\
\text { influence }\end{array}$ & $\begin{array}{l}\text { Delayed } \\
\text { influence }\end{array}$ & $\begin{array}{l}\text { Short-term } \\
\text { influence }\end{array}$ & $\begin{array}{l}\text { Long-term } \\
\text { influence }\end{array}$ \\
\hline \multirow{5}{*}{ Marketing } & Gaining & & & & \\
\hline & Development & & & & \\
\hline & Utilisation & & & & \\
\hline & Transfer & & & & \\
\hline & Retention & & & & \\
\hline \multirow{5}{*}{ R\&D } & & & & & \\
\hline & Gaining & + & & & \\
\hline & Development & & & & \\
\hline & Utilisation & + & & & \\
\hline & Transfer & & & & \\
\hline & Retention & & & & \\
\hline & & & & & \\
\hline \multirow{5}{*}{ Production } & Gaining & & & & \\
\hline & Development & & & & \\
\hline & Utilisation & & & & \\
\hline & Transfer & & & & \\
\hline & Retention & & & & \\
\hline
\end{tabular}


In this example the textual description of the intervention is "translated" into a formal specification: "+" in R\&D/Gaining/Immediate cell and another " + " in the $\mathrm{R} \& \mathrm{D} / \mathrm{Utilisation} / \mathrm{Immediate}$ cell. Both influences have a long term effect (the " $\mathrm{x}$ " symbols in the Long-term column).

Now please read our descriptions of a set of interventions and give their formal specifications or give your formal specification for the intervention if you don't agree with our description, following the procedure outlined in the example.

\section{Intervention 1}

Hire a high-class expert with new marketing knowledge on a temporary basis

\section{Description}

This intervention leads to knowledge gaining in the marketing domain, since a person with new knowledge is going to work in the marketing department. Additionally this intervention improves knowledge utilisation in the marketing department as this person applies his/her knowledge to the work. The intervention has an immediate effect, which persist over two game periods.

In the table below please give the formal specification of the influence of this intervention based on our description or, if you don't agree, based on your ideas about the influence(s) of the intervention. Please cross out the not-applicable option (yours, my own)

Formal specification (yours)/(my own)

\begin{tabular}{|c|c|c|c|c|c|}
\hline Domains & Processes & $\begin{array}{l}\text { Immediate } \\
\text { influence }\end{array}$ & $\begin{array}{l}\text { Delayed } \\
\text { influence }\end{array}$ & $\begin{array}{l}\text { Short-term } \\
\text { influence }\end{array}$ & $\begin{array}{l}\text { Long-term } \\
\text { influence }\end{array}$ \\
\hline \multirow{5}{*}{ Marketing } & Gaining & & & & \\
\hline & Development & & & & \\
\hline & Utilisation & & & & \\
\hline & Transfer & & & & \\
\hline & Retention & & & & \\
\hline & & & & & \\
\hline \multirow{5}{*}{$R \& D$} & Gaining & & & & \\
\hline & Development & & & & \\
\hline & Utilisation & & & & \\
\hline & Transfer & & & & \\
\hline & Retention & & & & \\
\hline & & & & & \\
\hline \multirow{5}{*}{ Production } & Gaining & & & & \\
\hline & Development & & & & \\
\hline & Utilisation & & & & \\
\hline & Transfer & & & & \\
\hline & Retention & & & & \\
\hline
\end{tabular}

\section{Comments:}

